Universidad | Campus Politécnica de Cartagena Internacional

\title{
DESARROLLO E IMPLEMENTACIÓN DE UN VEHÍCULO AUTÓNOMO. CLOUD INCUBATOR CAR
}

\section{Programa Doctorado}

Tecnologías Industriales

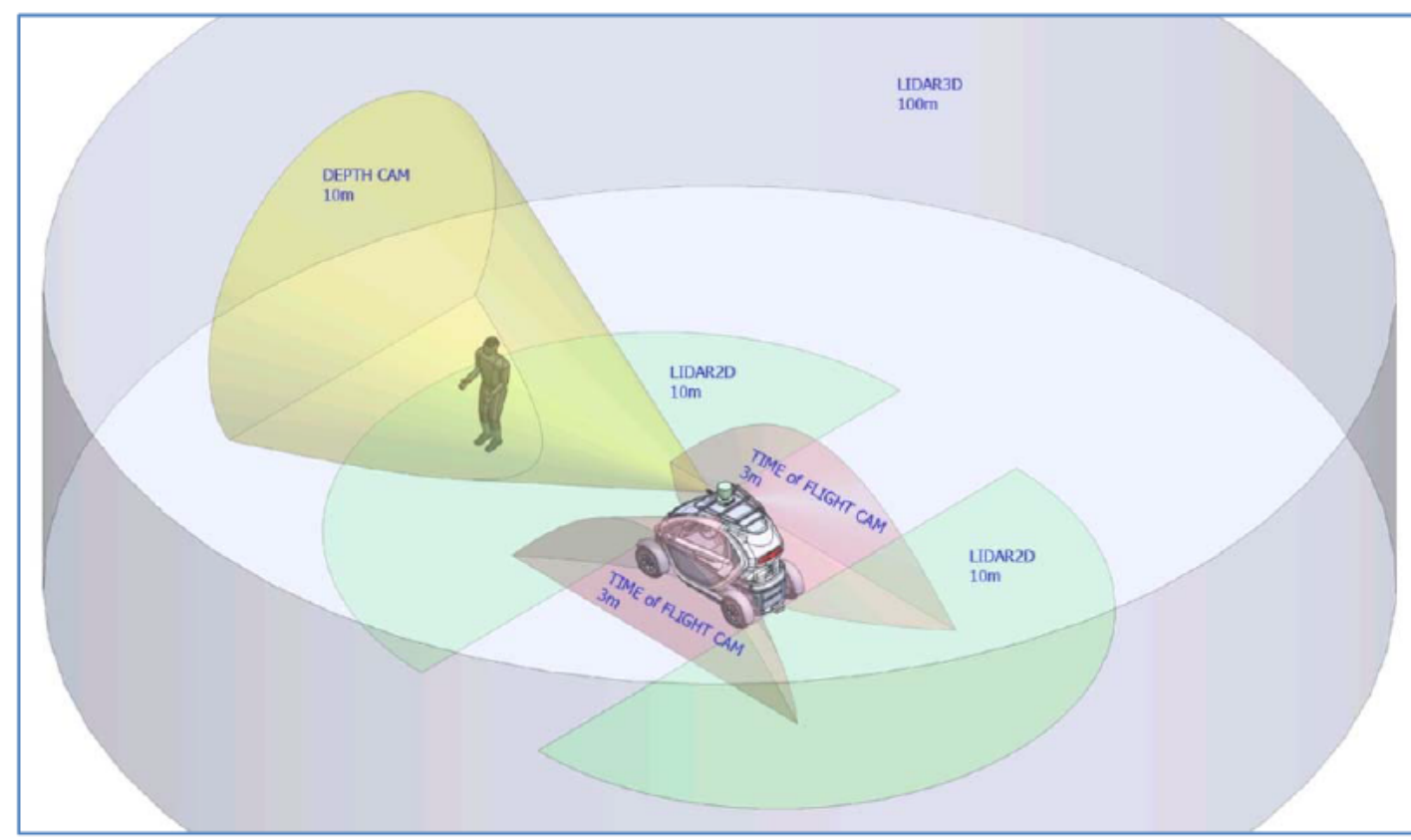

Autor: Raúl Borraz Morón

Director/es: Dr. Pedro J. Navarro Lorente

Dr. José Carlos Fernández Andrés

Cartagena Octubre 2020 


\section{CONFORMIDAD DE SOLICITUD DEAUTORIZACIÓN DE DEPÓSITO DE}

TESIS DOCTORAL POR EL/LA DIRECTOR/A DE LA TESIS

D/D ${ }^{\mathrm{a}}$. _ PEDRO J. NAVARRO LORENTE y JOSÉ CARLOS FERNÁNDEZ ANDRÉS Director/a de la Tesis doctoral_ DESARROLLO E IMPLEMENTACIÓN DE UN VEHÍCULO AUTÓNOMO. CLOUD INCUBATOR CAR_.

\section{INFORMA:}

Que la referida Tesis Doctoral, ha sido realizada por D/D ${ }^{\mathrm{a}}$ _ _ RAUL BORRAZ MORON _, dentro del Programa de Doctorado _ TECNOLOGIAS INDUSTRIALES

_, dando mi conformidad para que sea presentada ante el Comité de Dirección de la Escuela Internacional de Doctorado para ser autorizado su depósito.

La rama de conocimiento en la que esta tesis ha sido desarrollada es:

$\square$ Ciencias

$\square \quad$ Ciencias Sociales y Jurídicas

X Ingeniería y Arquitectura

En Cartagena, a_10_de_SEPTIEMBRE_de $\_2020$

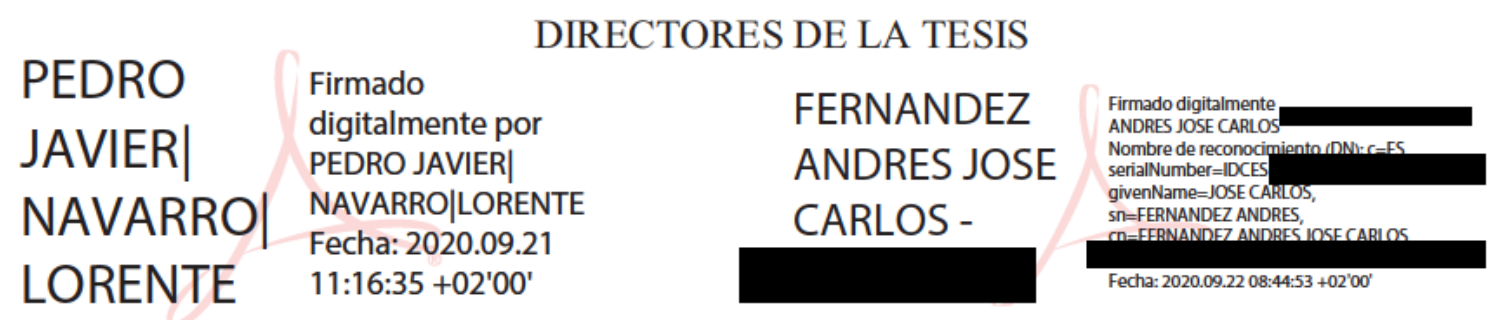

Fdo.: _ PEDRO J. NAVARRO LORENTE y JOSÉ CARLOS FERNÁNDEZ ANDRÉS 


\section{CONFORMIDAD DE DEPÓSITO DE TESIS DOCTORAL}

\section{POR LA COMISIÓN ACADÉMICA DEL PROGRAMA}

D/D ${ }^{\mathrm{a}}$. JUAN SUARDÍAZ MURO, Presidente/a de la Comisión Académica del Programa TECNOLOGÍAS INDUSTRIALES.

\section{INFORMA:}

Que la Tesis Doctoral titulada, “DESARROLLO E IMPLEMENTACIÓN DE UN VEHÍCULO AUTÓNOMO. CLOUD INCUBATOR CAR" , , ha sido realizada, dentro del mencionado Programa de Doctorado, por D/D ${ }^{\mathrm{a}}$._RAUL BORRAZ MORON_ _, bajo la dirección y supervisión del Dr./ Dra._ PEDRO J. NAVARRO LORENTE y JOSÉ CARLOS FERNÁNDEZ ANDRÉS

En reunión de la Comisión Académica, visto que en la misma se acreditan los indicios de calidad correspondientes y la autorización del Director/a de la misma, se acordó dar la conformidad, con la finalidad de que sea autorizado su depósito por el Comité de Dirección de la Escuela Internacional de Doctorado.

La Rama de conocimiento por la que esta tesis ha sido desarrollada es:

Ciencias

Ciencias Sociales y Jurídicas

$\mathrm{X}$ Ingeniería y Arquitectura

En Cartagena, a_09_de_SEPTIEMBRE__de_2020_

EL PRESIDENTE DE LA COMISIÓN ACADÉMICA

$\begin{aligned} & \text { JUAN| } \begin{array}{l}\text { Firmado } \\ \text { digitalmente }\end{array} \\ & \text { SUARDIA por JUAN| } \\ & \text { SUARDIAZ| } \\ & \text { Fdo: }\end{aligned}$





\section{AGRADECIMIENTOS}

a los integrantes del grupo de investigación del Laboratorio de Visión Artificial y Vehículos Inteligentes de la Universidad Politécnica de Cartagena (UPCT) por su importante apoyo en el desarrollo de esta tesis y especialmente a mis directores por guiar, revisar y acompañar todo el trabajo realizado. 



\section{RESUMEN}

La tesis propuesta se enmarca dentro del campo de la ingeniería de los vehículos autónomos y ha sido desarrollada en el seno del grupo de investigación División de Sistemas e Ingeniería Electrónica (DSIE) de la Universidad Politécnica de Cartagena.

Los vehículos autónomos están llamados a cambiar completamente la movilidad tal como la conocemos en la actualidad. Los avances en el software, junto con los sensores de última generación están propiciando un gran desarrollo de la denominada tecnología de conducción autónoma, que, sin duda, proporcionará grandes mejoras en la confiabilidad y el funcionamiento seguro de estos vehículos en condiciones del tráfico real.

La investigación sobre vehículos autónomos ha estado creciendo rápidamente en los últimos años y abarca diferentes dominios, incluidos la robótica, la informática, la ingeniería y la inteligencia artificial. Además, cabe señalar que tanto los fabricantes de automóviles como la industria auxiliar del automóvil han hecho avances científicos y técnicos de los que apenas divulgan públicamente detalles de sus enfoques o algoritmos, debido a la gran sensibilidad comercial de los mismos. Nos acercamos, sin duda, al momento en que la promesa, alimentada durante décadas, ver vehículos autónomos capaces de desenvolverse de manera segura a través del tráfico urbano y compartir las carreteras con otros participantes del tráfico se hará realidad.

Los vehículos autónomos constituyen una evolución prometedora de los actuales vehículos dotados de diferentes sistemas avanzados de asistencia al conductor, y se prevé que proporcionen una mayor seguridad vial, un flujo de tráfico eficiente y un menor consumo de combustible, al tiempo que mejoran la movilidad y, por lo tanto, el bienestar general.

Los vehículos autónomos perciben el entorno mediante técnicas complejas como visión artificial, láser, RADAR, LIDAR y sistemas de posicionamiento global. Los sistemas avanzados de control interpretan la información proporcionada por los mencionados sistemas de percepción con el fin de identificar la ruta apropiada, así como los obstáculos y la señalización relevante. Los vehículos autónomos actuales son capaces generalmente de recorrer carreteras previamente programadas y requieren de una adecuada reproducción cartográfica del terreno; en caso contrario se puede dar el caso que no sea posible la ejecución de un trayecto de forma coherente y en condiciones de seguridad.

Esta tesis contempla el estudio y desarrollo de una plataforma de investigación para aplicaciones de conducción autónoma, basada en un vehículo eléctrico comercial (Renault Twizy).

Para ello, se abordó una fase inicial consistente en el estudio, diseño, fabricación y montaje de elementos mecánicos (engranajes, motoreductores y drivers) necesarios para robotizar los componentes encargados de operar el volante, freno, acelerador y sentido de marcha.

A continuación, se realizó un análisis detallado de los diferentes sistemas de precepción disponibles en el mercado y se realizó una propuesta de sensorización de la plataforma para conducción autónoma El resultado de esta etapa ha sido la integración de un completo sistema de percepción del entorno compuesto por sensores ultrasónicos, LIDAR 3D, LIDAR 2D, cámaras de visión en diferentes espectros, cámaras de tiempo de vuelo (ToF), sistemas de posionamiento global (GPS) y sistemas de medida inercial (IMU). 
Se ha propuesto una arquitectura de control que abarca las fases de diseño, prototipado e implementación. Los sistemas del vehículo ( sistemas de percepción, servomotores, gestión de energía a bordo, etc,) son controlados mediante unidades de procesamiento hardware (NI CompactRIO 9082 / Xilinx FPGA y ordenadores embebidos Intel Core i7) y software (Labview) que proporcionan un sistema de control robusto y escalable y que permite una rápida implementación y prueba de algoritmos conducentes a la conducción autónoma.

Una vez que el vehículo posee la tecnología capaz de accionar automáticamente volante, freno, acelerador y sentido de marcha, visualizar y procesar el entorno cambiante a su alrededor, la siguiente fase fue diseñar algoritmos y software para la planificación global y local de posibles rutas, con las condiciones óptimas y seguras de circulación. Todo esto gobernado por un sistema inteligente de toma de decisiones.

Como prueba de concepto, se desarrolló e implementó una algoritmo de detección de peatones basado en el análisis de la nube de puntos obtenidos por medio del el sensor LIDAR Velodyne HDL64E. 


\section{ABSTRACT}

The proposed Ph.D. thesis is framed within the field of autonomous vehicle engineering and has been developed within the research group División de Sistemas e Ingeniería Electrónica (DSIE) of the Technical University of Cartagena.

Autonomous vehicles are called upon to completely change mobility as we know it today. Advances in software, along with state-of-the-art sensors, are leading to a major development of socalled autonomous driving technology, which will undoubtedly provide major improvements in the reliability and safe operation of autonomous vehicles in real traffic conditions. .

Autonomous vehicles perceive the environment using complex techniques such as computer vision, laser, radar, LIDAR, and global positioning systems. Advanced control systems interpret the information provided by the aforementioned perception systems in order to identify the appropriate route, as well as obstacles and relevant signaling. Today's autonomous vehicles are generally capable of traveling previously programmed roads and require adequate mapping of the terrain; otherwise it may be the case that it is not possible to execute a journey in a coherent and safe way.

This thesis contemplates the study and development of a research platform for autonomous driving applications, based on a commercial electric vehicle (Renault Twizy).

To do this, an initial phase consisting of the study, design, manufacture and assembly of mechanical elements (gears, servo motors and drivers) necessary to robotize the components responsible for operating the steering wheel, brake, accelerator and direction of travel was addressed.

Next, a detailed analysis of the different sensing systems available on the market was carried out and a proposal was made for sensorization of the platform for autonomous driving The result of this stage has been the integration of a complete system of perception of the environment composed of ultrasonic sensors, 3D LIDAR, 2D LIDAR, vision cameras in different spectra, time-offlight (ToF) cameras, global positioning systems (GPS) and inertial measurement systems (IMU).

A control architecture has been proposed that covers the design, prototype and implementation phases. Vehicle systems (perception systems, servo motors, on-board power management, etc.) are controlled by hardware processing units (NI CompactRIO 9082 / Xilinx FPGA and Intel Core i7 embedded computers) and software (Labview) that provide a robust and scalable control system which allows rapid implementation and testing of algorithms for autonomous driving.

As a proof of concept, a pedestrian detection algorithm was developed and implemented based on the analysis of the cloud of points obtained by a Velodyne HDL64E LIDAR sensor. 



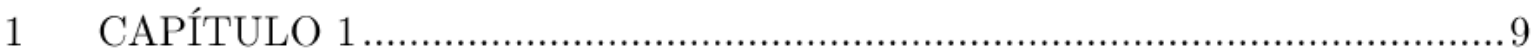

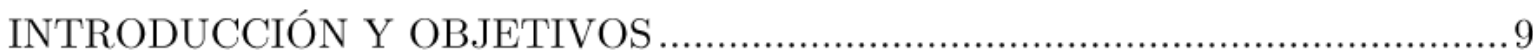

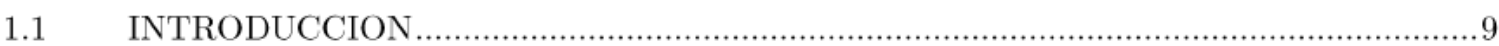

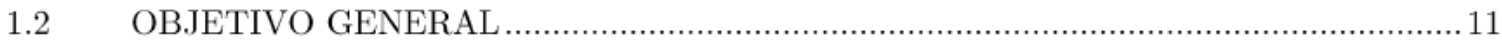

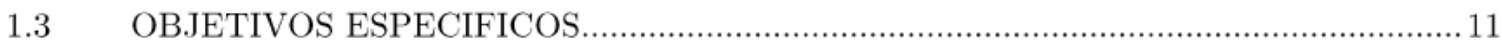

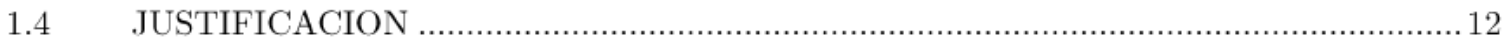

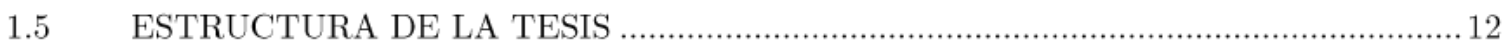

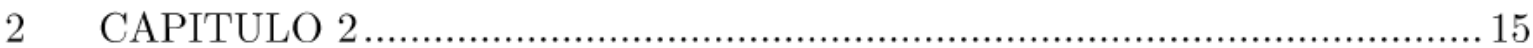

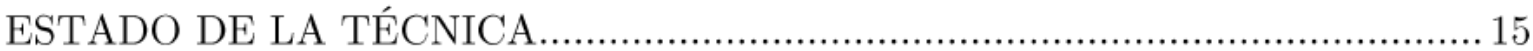

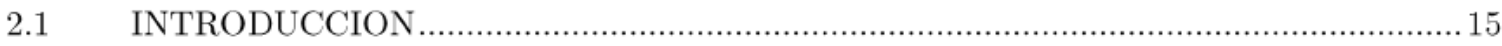

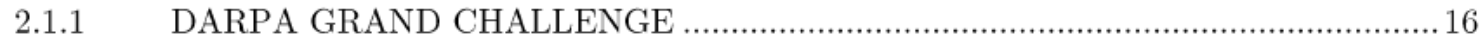

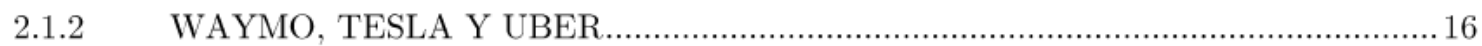

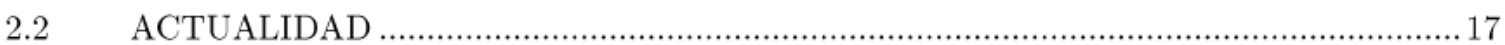

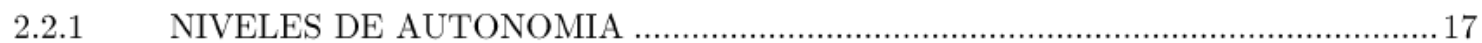

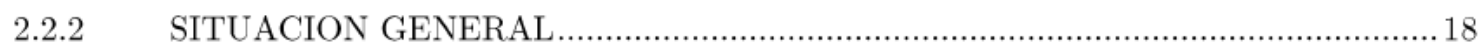

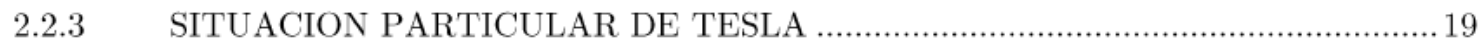

2.2.4 INTELIGENCIA ARTIFICIAL Y REDES NEURONALES ..................................... 19

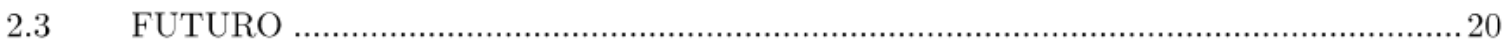

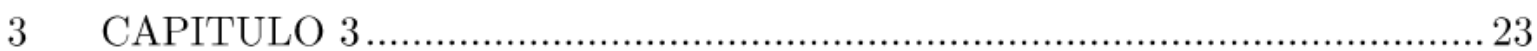

ROBOTIZACION DE CLOUD INCUBATOR CAR (CICAR) ........................... 23

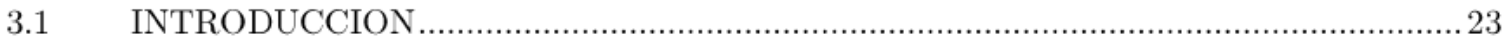

3.2 IMPLEMENTACION MECANICA CLOUD INCUBATOR CAR (CICAR) …...............22

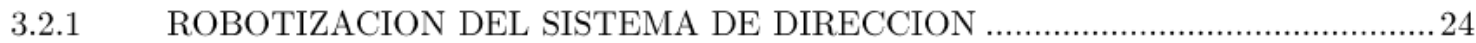

3.2.2 ROBOTIZACION DEL SISTEMA DE FRENADO …...........................................2

3.2.3 ROBOTIZACION DEL SISTEMA DE ACELERACION Y SELECCION DEL

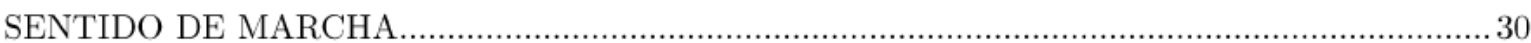

3.3 CONTROL TRAYECTORIAS. CONFIGURACIÓN ACKERMAN ……........................... 32

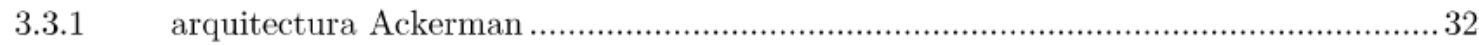

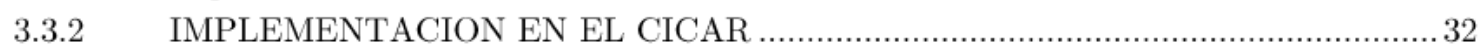


3.4 PLANIFICACION DEL MOVIMIENTO Y SISTEMA DE CONTROL .........................34

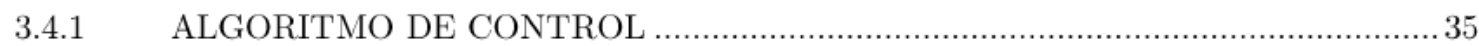

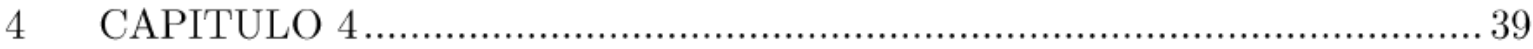

SISTEMAS DE PERCEPCION …........................................................... 39

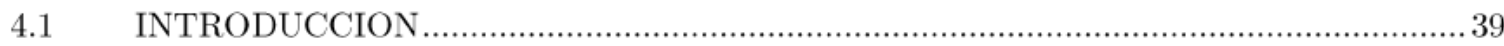

4.2 SISTEMA DE PERCEPCIÓN DEL ENTORNO PARA VAS ......................................4 40

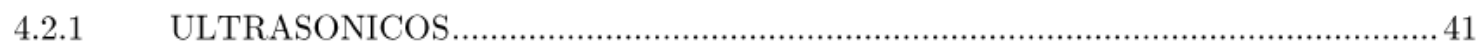

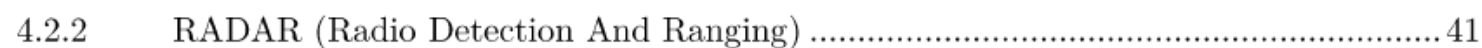

4.2.3 LIDAR (LASER IMAGING DETECTION AND RANGING) ..........................4 42

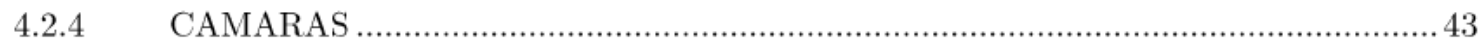

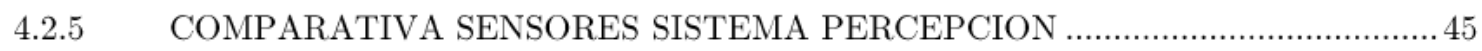

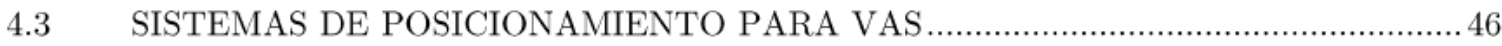

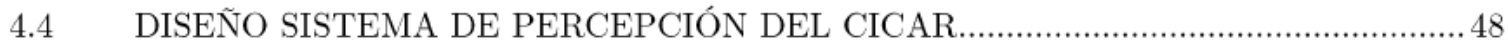

4.4.1 SISTEMA PERCEPCEPCIÓN DE CORTO ALCANCE (SRR) ............................51

4.4.2 SISTEMA PERCEPCEPCIÓN DE LARGO ALCANCE (LRR) ............................52

4.4.3 SISTEMAS DE POSICIONAMIENTO PARA VAS ….........................................5

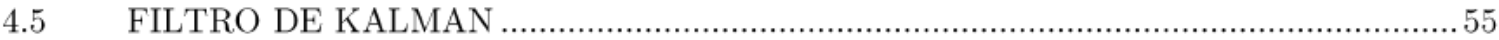

4.5.1 VARIABLES Y PARÁMETROS DEL FILTRO DE KALMAN............................55

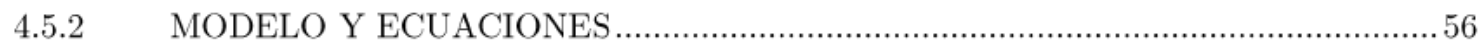

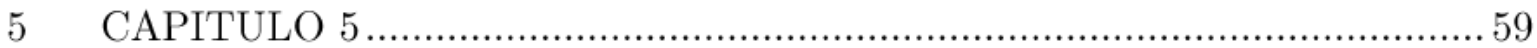

SISTEMA DE TOMA DE DECISION .......................................................... 59

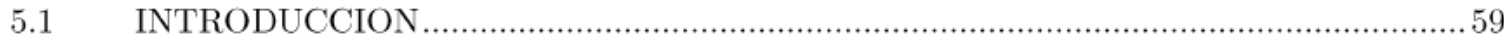

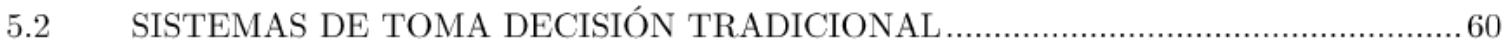

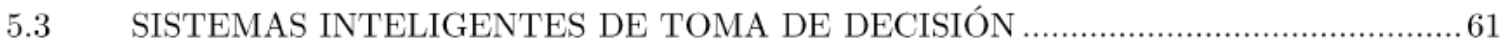

5.4 SIMULACIÓN Y HERRAMIENTAS SOFTWARE PARA DMS .................................6

5.5 DISEÑO DE UN SISTEMA DE TOMA DE DECISIÓN PARA CICAR .......................64

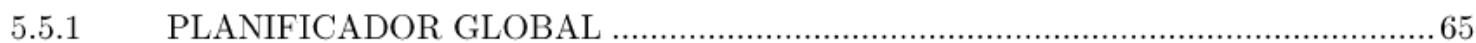

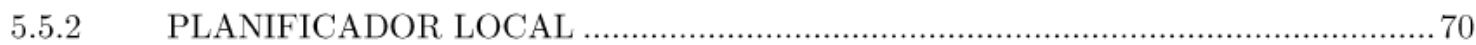


6 CAPITULO 6 75

ALGORITMO DE DETECCION DE PEATONES BASADO EN NUBES DE PUNTOS 75

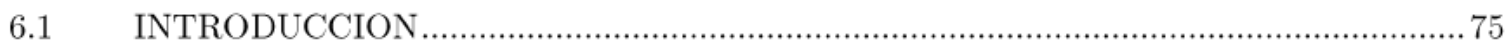

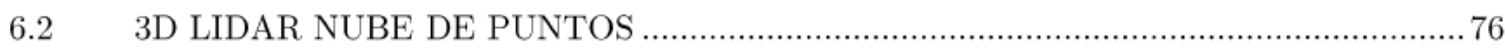

6.3 ALGORITMO DE DETECCIÓN DE PEATONES …...............................................

6.3.1 PASO 1. RECOPILACION DATOS PARA PROYECCIONES AXNOMETRICAS XY YZ XZ 77

6.3.2 PASO 2: GENERACIÓN PROYECCIONES AXONOMETRICAS XY, YZ Y XZ..77

6.3.3 PASO 3: GENERACIÓN Y PREPROCESADO IMÁGENES BINARIAS

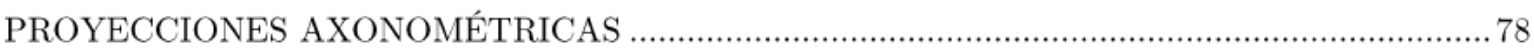

6.3.4 PASO 4: CÁLCULO DEL VECTOR DE CARACTERÍSTICAS …...........................79

6.3.5 PASO 5: ALGORITMO DE APRENDIZA.JE AUTOMÁTICO …….........................82

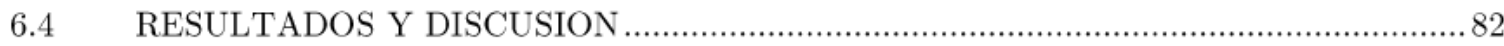

6.4.1 RENDIMIENTO DE ALGORITMOS DE APRENDIZAJE AUTOMÁTICO ...........84

6.4.2 RENDIMIENTO DEL ALGORITMO COMPLETO DE DETECCIÓN DE PEATONES 86

$7 \quad$ CAPITULO 7 89

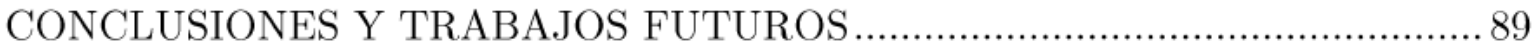

7.1 APORTACIONES Y CONCLUSIONES .89

7.2 TRABAJOS FUTUROS .92

8 REFERENCIA BIBLIOGRÁFICA …................................................... 93

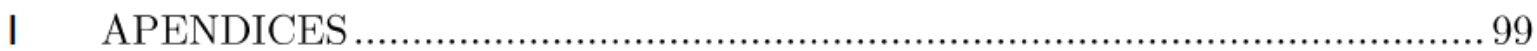

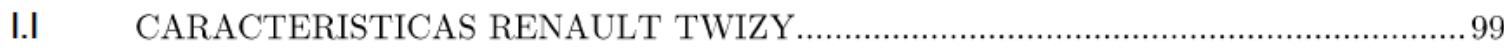

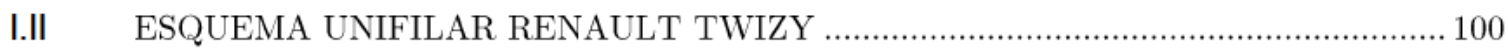

I.III HOJAS DE CARACTERISTICAS DEL HARDWARE INSTALADO …....................... 101

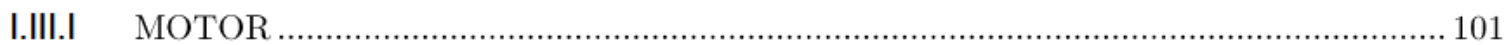

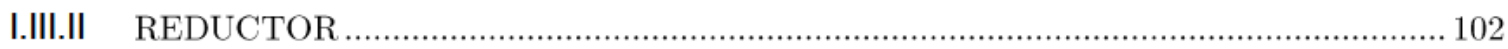

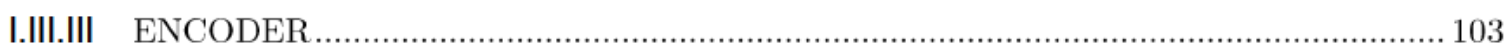

I.III.IV DRIVER 


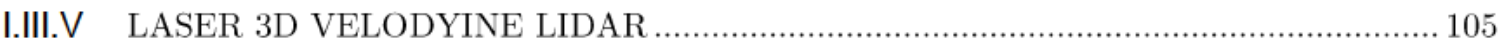

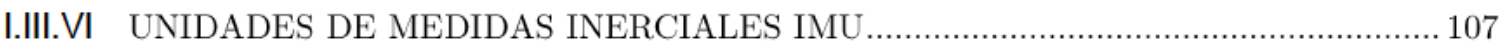

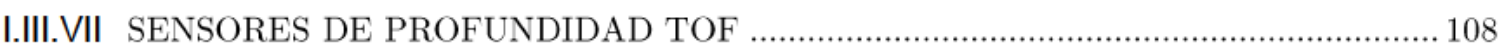

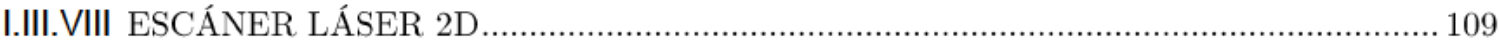




\section{ÍNDICE DE TABLAS}

Tabla 1. Valores muestreados de tensión acelerador..... .32

Tabla 2. Resumen principales características sensores utilizados sist percepción de VAS ...............45

Tabla 3. Algunas plataformas para el desarrollo de conducción autónoma ...................................47

Tabla 4. Características de los sensores del sistema de percepción. ...............................................4 48

Tabla 5. Propiedades de diferentes tecnologías de detección ...................................................50

Tabla 6. Sistemas de corto alcance montados en el CICar...................................................51

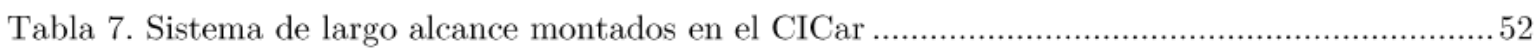

Tabla 8. Distancias ejes de referencia sistema percepción CICar ................................................54

Tabla 9. Resumen principales características de las plataformas de simuladores para VAs ............6 63

Tabla 10. Cálculo de ángulos dependiendo de la dirección ......................................................66

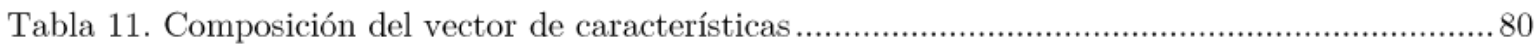

Tabla 12. Parámetros de configuración de kNN, NBC y SVM ................................................. 82

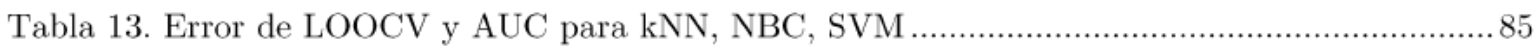

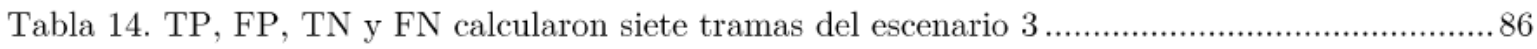

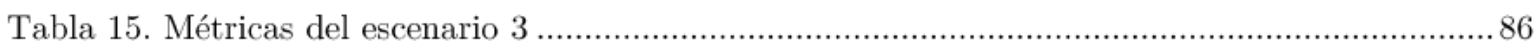

Tabla 16. Comparación del método propuesto con otros autores................................................ 87

\section{ÍNDICE DE FIGURAS}

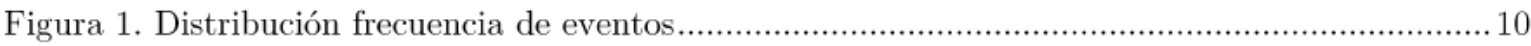

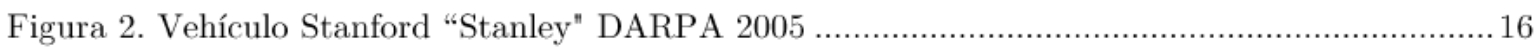

Figura 3. Toma de decisiones morales y éticas propias de los VAs............................................21

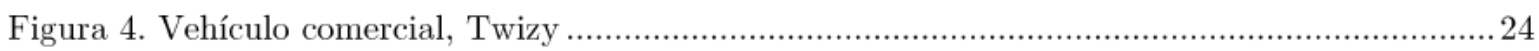

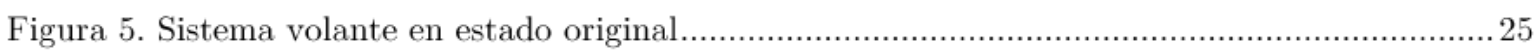

Figura 6. Diseño 3D de la solución adoptada para volante .......................................................25

Figura 7. Imagen del sistema automatizado de volante ......................................................26

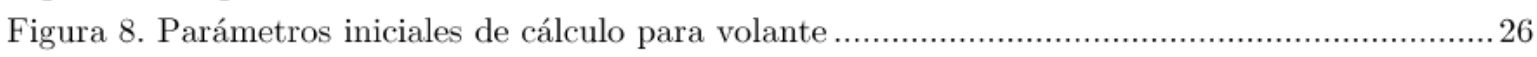

Figura 9. Diseño 3D para diseño de engranajes de volante ..................................................27

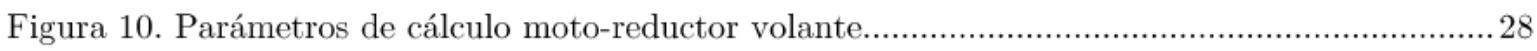

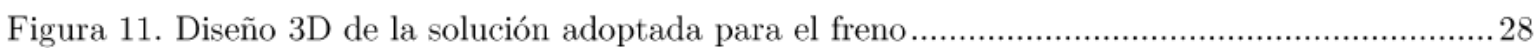

Figura 12. Parámetros iniciales de cálculo para freno ...............................................................29

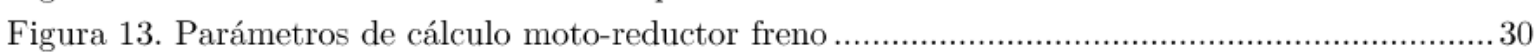

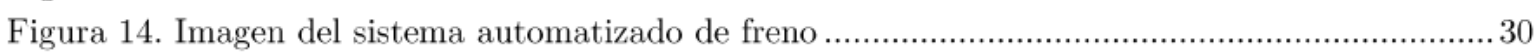

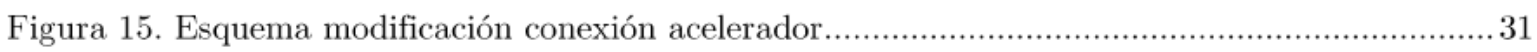

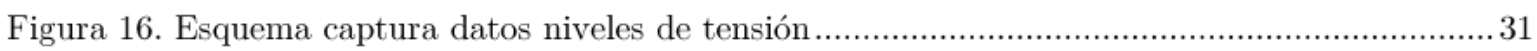

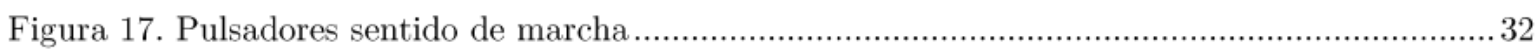

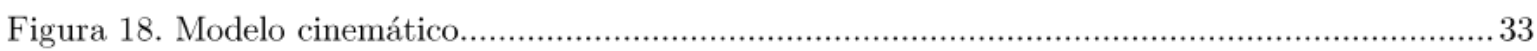

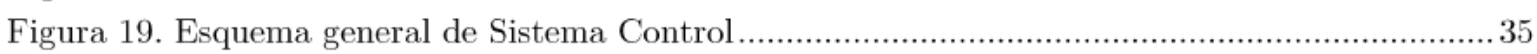

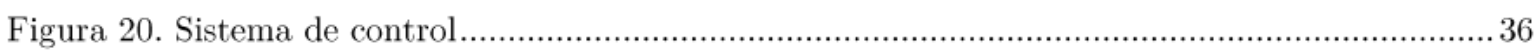

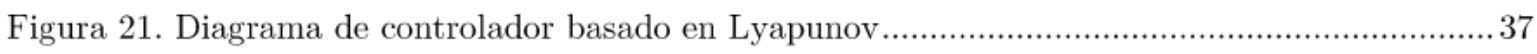

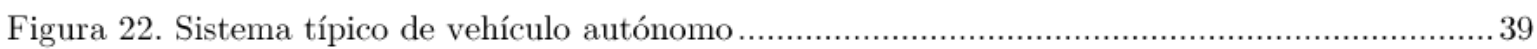

Figura 23. Descripción general espectros utilizados sistema percepción vehículos autónomos..........40

Figura 24. (a) Sensores ultrasónicos automóviles Bosh; (b) Sist asistencia estacionamiento Audi ...41

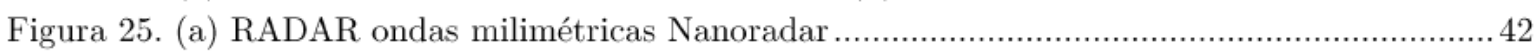

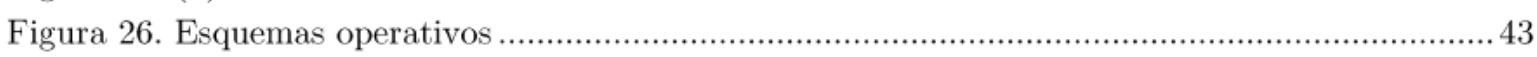

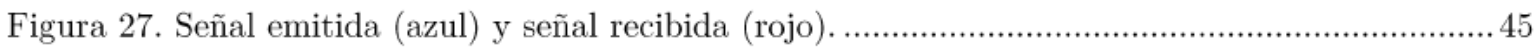

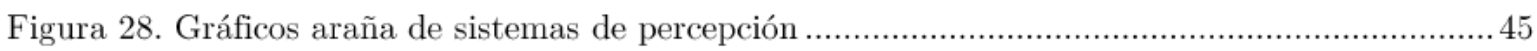

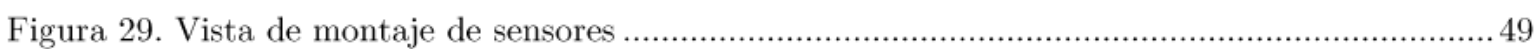


Figura 30. Diagrama de rango de los sensores montados en el CICar ....................................50

Figura 31. Sistemas de referencia de sensores en el vehículo autónomo......................................51

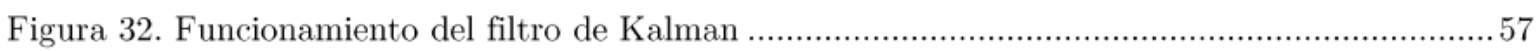

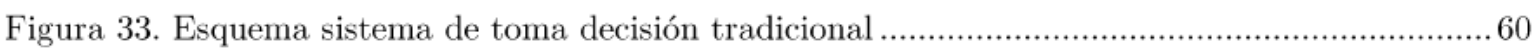

Figura 34. Arquitectura funcional de conducción autónoma ...................................................61

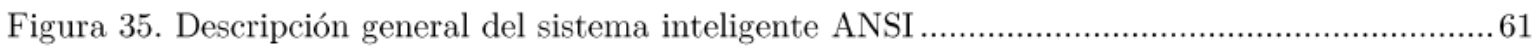

Figura 36. Distribución de los componentes de arquitectura de alto nivel del CICar....................64

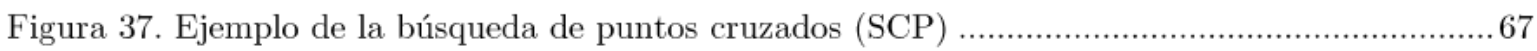

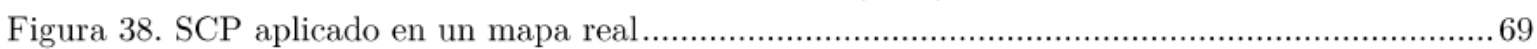

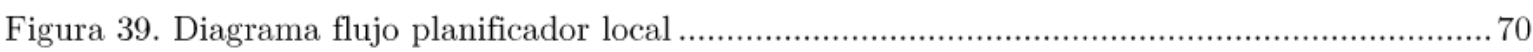

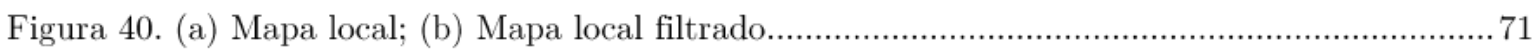

Figura 41. Waypoints (WPi, WPi+1), ROI y puntos clave en dos escenarios ..........................71

Figura 42. Algoritmo generador de trayectorias ................................................................. 72

Figura 43. (a) escenario 1; (b) escenario 2; (c) escenario 3; (d) escenario 4 (nCurves .....................73

Figura 44. escenario 1 nCurves $=30$, distancia de seguridad $=1 \mathrm{~m}$, criterios $=$ ángulo máximo....74

Figura 45. Muestra peatonal capturada software sobre un marco del 3D LIDAR.........................77

Figura 46. Proyecciones axonométricas XY, XZ y YZ normalizadas muestras de peatones .............78

Figura 47. Imágenes binarias generadas de proyecciones XY, XZ y YZ muestras de peatones ........79

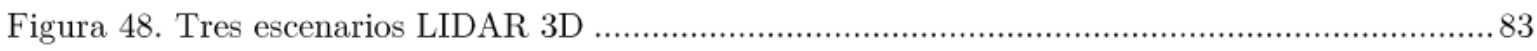

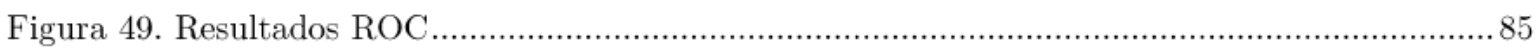

Figura 50. Algoritmo de detección de peatones en área de tráfico real................................... 87

\section{SIGLAS}

CICar, CLOUD INCUBATOR CAR

ECUs, Unidades de control electrónicas

FOV, Campo de visión reducido

VIS, Visibles

IR, Infrarrojas

LVC, Vivo, virtuales y constructivas

RTK, Cinemática en tiempo real

SAV, Vehículos autónomos compartidos (SAV)

VA, Vehículos autónomos

VI, Vehículo Inteligente

ATO, Automatic Train Operation

RTK, Unidad cinemática en tiempo real

PPS, Sistema planificación de ruta (pps, path planning system)

CCD, Cámara de visión frontal

NHTSA, Autoridad de Tráfico de Estados Unidos

IMU, Unidad de Medidas Inerciales

V2V, Vehículo a vehículo

V2I, Vehículo a infraestructura

ADAS, sistemas avanzados de asistencia al conductor

DF, Data Fusion

UAV, Vehículos aéreos no tripulados

IDMS, Sistema toma de Decisión Inteligente (Intelligent-Decision-Making Systems)

PPS, Sistema planificación de ruta (path planning system)

MLA, Machine Learning Algorithms 


\section{CAPÍTULO 1}

\section{INTRODUCCIÓN Y OBJETIVOS}

\subsection{INTRODUCCION}

Uno de los últimos avances tecnológicos prometidos en la ola de progreso en la que estamos inmersos es el coche autónomo. Una sofisticada máquina capaz de interpretar el complejo escenario en el que se desarrolla el tráfico y desenvolverse en el mismo, adaptándose a los múltiples desafíos que puedan surgir.

Los vehículos autónomos llegan con promesas muy atractivas; por mencionar solo un par de ellas: a) una movilidad sin víctimas; si un conductor humano comete un error durante la conducción en una localización determinada, , ese error es muy posible que se repita por otro humano en la misma situación [1]; pero si un coche autónomo comete ese error en la misma localización, el resto de vehículos autónomos podrán aprender de ese error y no volver a cometerlo jamás; b) una movilidad independiente al alcance de personas que no disponen de ella actualmente, como invidentes, ancianos, discapacitados, etc.

Esto tiene un potencial transformador de tal calado que muy difícilmente la sociedad y la industria lo podían dejar pasar. Se observa como todas las grandes empresas automovilísticas (Ford, Renault, Toyota, Waymo, Uber, Mercedes, Audi o Tesla) han invertido durante los últimos años sumas millonarias en investigar y desarrollar sus propios prototipos de vehículos autónomos.

Aunque hay fabricantes (como Tesla) que ya ofrecen paquetes de conducción autónoma completa en sus vehículos, la realidad es que tal oferta resulta engañosa. Los coches de Tesla equipados con el sistema FSD (Full Driving System), sólo aportan, al sistema Autopilot que viene por defecto en estos coches, una serie de funcionalidades extra de navegación autónoma, como por ejemplo:

- cambio automático de carriles bajo acción del conductor

- aparcamiento automático en parkings

- navegación automática en entrada y salida de autopistas

Se trata de un conjunto de sistemas de asistencia a la conducción que no se puede considerar conducción autónoma completa. Esto se ha criticado bastante a la compañía Tesla, que se defiende diciendo que ellos ofrecen un vehículo con el equipamiento necesario, para poder implementar una conducción autónoma total y a través de actualizaciones de software y avances en la legislación, permitirá que el coche vaya logrando una mayor autonomía hasta alcanzar el nivel 5.

Google en 2012 anticipó que en cinco años el coche autónomo sería una realidad para el consumidor, que de momento en 2019, todavía sigue siendo un proyecto piloto en pruebas. Ford en 2016 anunció que en cinco años se encontraría produciendo coches sin volantes ni pedales. Un par de años después declaró que el problema era más complicado lo que esperaban inicialmente. General Motors anunció que este objetivo requiere de más tiempo. 
El problema es bastante complejo, no sólo porque conducir implica saber adaptarse a diferentes tipos de carreteras y meteorología, lluvia, niebla, atardecer o estilo de conducción, ya sea en autopista o en ciudad, sino también porque todo esto ha de funcionar en coordinación con otros conductores, peatones y cualquier elemento impredecible que pueda aparecer en escena.

Para resolverlo podemos [1] dividir el problema en :

- percepción y fusión de datos

- predicción y control

- acción toma de decisiones

Se denomina percepción a la capacidad para conseguir que un coche sea capaz de percibir todos los elementos de su entorno, contando con los sensores necesarios. En este apartado es donde se vive una apasionante batalla, la batalla de las cámaras contra el LIDAR.

La labor de percepción y fusión de datos, no acaba en elegir el tipo de sensor necesario a instalar, ahora toca dar sentido a todos estos datos y entender su contenido. Una aproximación que gana terreno es el diseño y uso de diferentes tipos de redes neuronales que son entrenadas para resolver diferentes sub problemas; así, por ejemplo, se pueden resolver problemas de segmentación para distinguir los diferentes tipos de elementos percibidos en escena (detectar cruces, semáforos, prolongaciones de carretera, etc...). Esto añade un extra de información que será relevante para la siguiente fase, la toma de decisiones.

En la fase de predicción y control se abre paso el denominado "Machine Learning", una infraestructura de etiquetado de datos que permite una enorme labor de clasificación y segmentación de información muy variada y compleja. El hándicap es, ¿quién clasifica este etiquetado?.

En la gráfica (Figura 1) se representa los diferentes tipos de eventos que pueden ocurrir en carretera y su frecuencia.

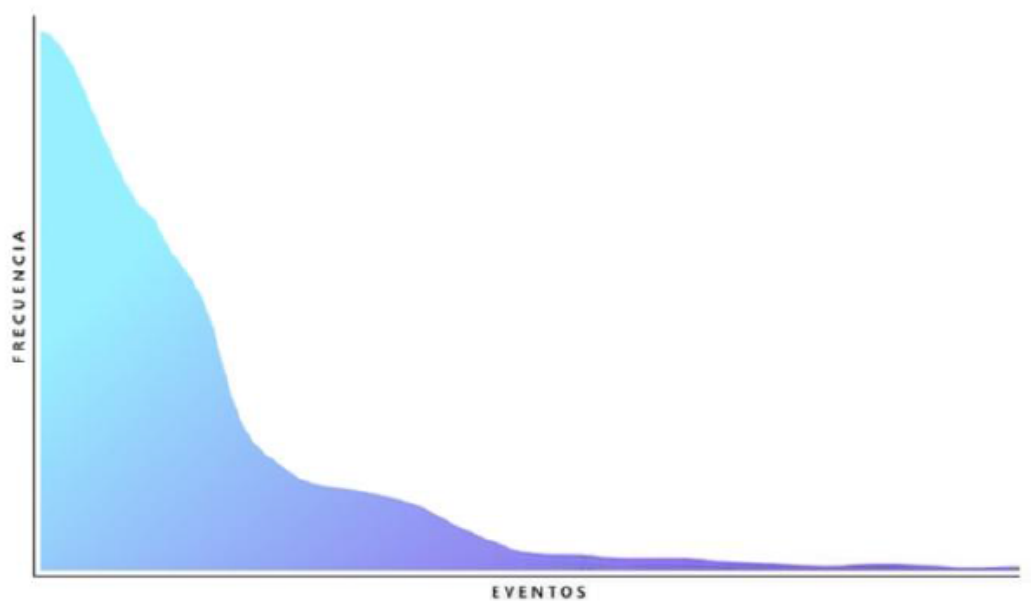

Figura 1. Distribución frecuencia de eventos

Un gran cúmulo de eventos que se repiten que son frecuentes, estarían situados a la izquierda de la distribución como por ejemplo:

- un coche adelantando

- una curva a la derecha

- una salida autopista, etcétera 
El problema es que la distribución también contiene un gran número de eventos raros y muy diversos, que también han de ser aprendidos y se encuentran en la parte derecha de la distribución.

La última fase es la de toma de decisiones, la que proporciona la respuesta del sistema; se trata de obtener aquellas acciones que deberá de realizar el vehículo para su control, acciones que serán ejecutadas por un controlador, girando el volante, activando pedales o señalizando correctamente los movimientos inminentes. En esta fase se puede tomar datos reales, mediante la captura de los sensores, bajo la supervisión humana y que el algoritmo aprenda estas acciones por imitación.

En este sentido, resulta interesante la estrategia de Tesla que, con el fin de penetrar en el mercado y poner el mayor número de unidades en las carreteras mediante la reducción de costes, , ha puesto en operación una flota de vehículos que cada día sigue sumando horas y horas de datos de conducción, lo que resulta clave para alimentar su sistema de aprendizaje por imitación.

\subsection{OBJETIVO GENERAL}

Estudio, diseño y desarrollo de una plataforma de investigación para aplicaciones de conducción autónoma, basada en un vehículo eléctrico comercial (Renault Twizy), que proporcione un sistema de control robusto y escalable y que permite una rápida implementación y prueba de algoritmos conducentes a la conducción autónoma, y que pueda ser usada por los estudiantes de máster y doctorado en sus trabajos de tesis.

\subsection{OBJETIVOS ESPECIFICOS}

- Realizar la respectiva revisión bibliográfica.

- Diseño, fabricación y montaje de elementos mecánicos para lograr automatizar los controles principales de conducción (volante, freno, acelerador y sentido de marcha).

- Estudio, selección, adquisición y montaje de sistemas de precepción para sensorizar el vehículo autónomo y auto posicionamiento.

- Estudio, selección, adquisición y montaje de hardware para controlar los sistemas mecánicos y sensores implementados.

- Desarrollo de aplicaciones, software y algoritmos para toma de decisiones, planificación global y local de rutas.

- Desarrollo de una aplicación, software y algoritmo novedoso para detección de peatones basado en nube de puntos obtenidos con el sensor LIDAR Velodyne HDL64E. 


\subsection{JUSTIFICACION}

A lo largo de la historia, la fuerza o la mente del ser humano ha sido reemplazada en algunas tareas por máquinas, de menor a mayor complejidad. Cuando se habla de coches autónomos, nos referimos a ceder el control de la conducción a un ordenador muy potente, con todo lo que eso implica. El ser humano no tiene aun la capacidad de predecir todas las situaciones que se pueden dar en muchos ámbitos de la vida; entre ellos, en la tarea de conducir un automóvil.

El piloto automático ayuda desde hace décadas a la navegación en aviones, trenes (ATO Automatic Train Operation) [2] y barcos. A diferencia de estos escenarios, la carretera es mucho más compleja por la elevada cantidad de obstáculos y vehículos que pueden encontrarse en ruta. Por lo tanto, es un problema de una dimensión superior.

Una de las ventajas que presenta la conducción automática, es la ausencia de emociones en la conducción. El "conductor" no va a picarse, ofenderse, ser más temerario o más torpe. Siempre va a conducir de la misma forma. Eliminar los errores humanos de la conducción ya hace mucho por aumentar la seguridad vial.

Uno de los motivos fundamentales para extender la conducción automatizada es reducir drásticamente el número de accidentes, los heridos graves y los fallecidos. Los programas informáticos son inmunes a las distracciones, la fatiga o los problemas psicomotrices, tienen una tasa de errores muy baja, y cuando los tienen, suele ser por un defecto en su programación, la cual realizan seres humanos.

\subsection{ESTRUCTURA DE LA TESIS}

El presente documento se divide en siete capítulos, referencia bibliográfica y un apéndice. El capítulo 1, a modo de introducción, incluye una breve presentación del marco actual y los sistemas de ayuda a la conducción, además de los objetivos y la estructura de la tesis.

En el capítulo 2 se desarrolla el estado de la técnica, que consta de una introducción a la actualidad de la conducción autónoma, breve descripción de la situación general y particular de sistemas de ayuda a la conducción existentes y análisis de las perspectivas de futuro.

En el capítulo 3 se detalla el diseño y montaje mecánico de los sistemas necesarios para el control del vehículo (volante, freno, acelerador y sentido de marcha), su robotización. Además se describe un algoritmo de planificación del movimiento y el sistema de control de trayectoria del vehículo.

En el capítulo 4 se explica y describe el sistema de percepción necesario para conocer el entorno del vehículo autónomo, instalado en el mismo. Se relaciona las diferentes especificaciones, características y una comparación de estos sensores, desde diferentes puntos de vista. El capítulo concluye con la descripción de los sistemas de posicionamiento para el vehículo y la descripción de la aplicación del Filtro de Kalman.

Una vez que se ha logrado la robotización de volante, freno, acelerador, sentido de la marcha y se conoce el entorno exterior que rodea al vehículo objeto, el capítulo 5 describe el sistema de toma de decisión y un algoritmo para realizar una conducción segura y una planificación de ruta capaz de salvar y evitar obstáculos. 
En capítulo 6 enfoca el desarrollo de un algoritmo de detección de peatones basado en nubes de puntos, tres mil marcos recopilados por LIDAR Velodyne HDL64E y procesados en la fase inicial.

El último capítulo 7, se dedica a conclusiones y trabajos futuros.

La estructura de la tesis finaliza con la referencia bibliográfica y un apéndice. 


\section{CAPITULO 2}

\section{ESTADO DE LA TÉCNICA}

\subsection{INTRODUCCION}

Hace 10 años el concepto de vehículo autónomo capaz de circular en entornos reales de tráfico parecía un objetivo lejano. Se daban a conocer implementaciones en entornos cerrados y se estudiaba como rentabilizar esta tecnología [3]. La llegada de los primeros vehículos sin conductor a nuestras ciudades y carreteras ha supuesto un gran cambio, comprable quizás al experimentado por los londinenses al ver por primera vez vehículos propulsados por motores en lugar de caballos, a finales del siglo XIX. Al igual que aquellos sorprendidos ciudadanos observaron aterrados los primeros accidentes mortales protagonizados por los nuevos automóviles, cuando se suponía que la tracción mecánica pondría a la máquina bajo el control total del conductor, hoy nos sorprenden noticias de accidentes de vehículos autónomos, cuando suponíamos que la conducción autónoma pondría a la conducción de un automóvil bajo el control de sistemas automáticos infalibles.

La conducción autónoma se puede ver como problema, misión, posibilidad de negocio, etc. Pero todos los actores involucrados coinciden en que se supone una tecnología disruptiva con la que trata de mejorar el acceso a la movilidad, así como su eficiencia y seguridad. Permitirá desplazarse en medios de transporte individuales a personas que no tienen la capacidad de hacerlo conduciendo por sí mismos a la vez que incrementará la productividad en el tiempo que usamos para desplazarnos y, por supuesto, disminuirá la accidentalidad. Este último aspecto resulta de un interés social evidente, si consideramos que según las estadísticas de siniestralidad. Cada 23 segundos muere una persona en el mundo por accidente de vehículo.

La investigación y diseño de robots móviles ha crecido desde los años 70 de manera exponencial. Esta gran evolución ha permitido que hoy en día, éstos robots móviles se puedan utilizar para navegar en distintos terrenos: terrestres, aéreos, subacuáticos, etc., con aplicaciones también muy amplias como: reconocimiento de terreno, automatización de procesos, búsqueda y rescate de personas en lugares de difícil acceso, exploración planetaria o para ampliar el rango de trabajo de robots manipuladores. Los vehículos autónomos tienen la oportunidad de transformar la sociedad actual.

El crecimiento de la movilidad en esta década y, sobre todo, la preponderancia del modo de transporte por carretera sobre los demás, tiene algunos efectos negativos en diferentes áreas: congestión, contaminación y accidentes, principalmente [4]. En particular, en el ámbito europeo:

- Aproximadamente el 10\% de la red vial está afectada por la congestión diaria.

- El transporte es el sector que más ha crecido en demanda de energía, el 83\% corresponde al transporte por carretera, que a su vez tiene una relación directa con las emisiones de CO2.

- Los vehículos son la principal fuente de contaminación en las ciudades y el $20 \%$ de las ciudades sufren niveles inaceptables de ruido.

- Cada año, en la Unión Europea, hay alrededor de 4.000 muertes y 1,7 millones de heridos en accidentes de tráfico. En el mundo 1,3 millones de muertes. 
- En términos económicos, los efectos de la congestión representan el 0,5\% del PIB, el impacto ambiental representa el $0,6 \%$, y los accidentes representan el 1,5\%.

\subsubsection{DARPA GRAND CHALLENGE}

En 2004, en el desierto de Mojave, California, se inició el Gran Desafío DARPA (Defense Advanced Research Projects Agency), con el objetivo de demostrar la viabilidad técnica de los vehículos autónomos (VAs) sin actuación humana. Durante la primera edición, el recorrido era de 142 millas y participaron 15 vehículos, pero ningún vehículo logró terminar la prueba; solo recorrieron el $5 \%$ del trayecto.

El año siguiente se repitió la carrera, participaron 23 vehículos y solo 5 llegaron a la final. El robot de la Universidad de Stanford "Stanley" [5] fue el ganador, completando la prueba en 6 horas y 53 minutos.

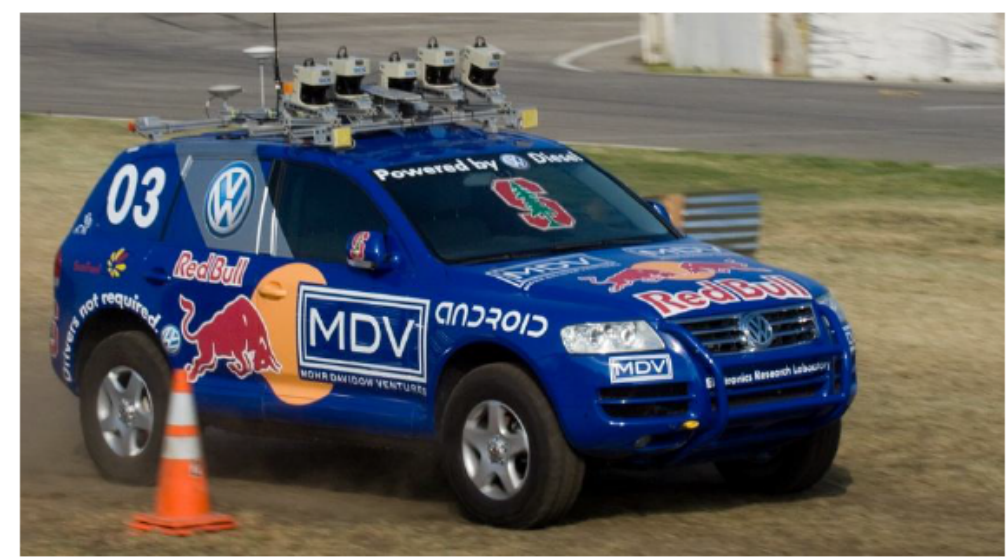

Figura 2. Vehículo Stanford "Stanley" DARPA 2005 (fuente: internet)

La siguiente carrera se celebró en el año 2007 [6], en una base de la fuerza aérea al sur de California. Fue re-bautizada "2007 Urban Challenge", pues incorporaba un tramo urbano, cumpliendo las normas de tráfico reales. El equipo "Carnegie Mellon's Tartan Racing" ganó la carrera, en poco más de cuatro horas.

\subsubsection{WAYMO, TESLA Y UBER}

En 2018 Waymo completó con sus vehículos en varios ciudades y entornos 10 millones de millas en modo autónomo. Este hito resultó un gran paso y despegue para la conducción autónoma que crece día a día.

Otra fabricante que ha ganado protagonismo en los última años es Tesla; mil millones de millas alcanzadas en conducción semiautónoma con piloto automático, con la considerable obtención de datos con la perspectiva futura de aprendizaje automático mediante redes neuronales.

En marzo 2018 sucedió el primer accidente con un vehículo autónomo de Uber en Tempe, Arizona, golpeando a un peatón con resultado mortal. También en 2018 en Mountain View, California, se estrelló un coche de Tesla que circulaba en modo piloto automático muriendo su conductor. 


\subsection{ACTUALIDAD}

Cada día aparecen noticias sobre vehículos autónomos y en este capítulo se va a desvelar como está el estado de la técnica de esta tecnología.

Las principales empresas tecnológicas del mundo están apostando por llevar a cabo sus propios prototipos. Algunas, como Apple, ya han abandonado el intento y otras, como Waymo (Google), se han centrado en el software.

Un coche autónomo o vehículo autónomo, es un sistema complejo donde se involucran muchas tecnologías y entran en juego diferentes disciplinas como la mecánica o visión artificial. En este apartado se revisan estos aspectos.

Un coche autónomo debe saber dónde está en todo momento, su auto localización es de vital importancia. Además, debe ser capaz de controlar todo el entorno que rodea el coche, objetos, personas, los otros coches, etc...para desarrollar su función principal, es que sea capaz de llevarnos de un punto A a un punto B.

Dentro de este sector, existen diferentes niveles de autonomía, desde el coche que conducimos todos, hasta el coche capaz de hacer largos recorridos, e incluso repostando de forma autónoma.

Existen varios programas activos de conducción autónoma, con conocidas empresas involucradas en su desarrollo, como por ejemplo Waymo (Google), Uber, Mercedes, Daimler AG, BMW, Renault, Ford o Volvo, así como LIDAR Velodyne, Bosch o Delphi, en el área de componentes y electrónica.

\subsubsection{NIVELES DE AUTONOMIA}

Los cinco niveles de la conducción autónoma es una cuestión que es referida de forma imprecisa frecuentemente, aunque están perfectamente definidos por la SAE (Society of Automotive Engineers) [7].

En el nivel 0, no hay asistencia a la condición, es decir, no hay apoyo de ningún tipo.

En el nivel 1 el vehículo ayuda al conductor en la conducción, por ejemplo, el sistema de control del crucero activo (que llega al mercado en el año 98)

El nivel 2 automatiza tanto la forma longitudinal de movimiento como la transversal, "High Way Asist". En este caso el conductor siempre tiene que estar al mando del vehículo, con las manos en el volante. Ejemplos son el control de crucero activo y el control lateral centrado de carril. Muchas marcas comerciales disponen de este nivel de automatización en la actualidad.

La verdadera conducción autónoma empieza con el nivel 3, ya no se habla de ayuda a la conducción. Se automatiza también el movimiento de modo longitudinal y transversal, pero no necesita en este caso tener las manos en el volante. El conductor puede responder a una llamada, responder a un correo electrónico, etc, pero si el vehículo lo solicita, el conductor debe retomar la conducción en unos segundos. En el mercado el Audi A8 se ha promocionado con este nivel, pero aun no se encuentra dentro del marco legal.

En el nivel 4 el vehículo puede conducir, la mayor parte del tiempo, en modo autónomo en ciertos entornos, ya no es necesario que el conductor esté atento a la conducción, en este caso el sistema no debe devolver en el control. El sistema debe ser robusto. El conductor podrá ver películas, incluso descansar o dormir. Este nivel está limitado por las condiciones en donde puede funcionar, en entornos muy controlados, por ejemplo en autopistas. Cuando dichas condiciones 
estén a punto de finalizar, el vehículo cede el control al conductor, que en caso de que no responda, llevará a que el vehículo estacione en una zona segura.

El nivel 5 el control de la conducción no se devolverá bajo ninguna condición, funciona en todas las condiciones, cualquier tipo de vía e incluso en cualquier situación ambiental. No es necesario disponer de volante ni pedales.

\subsubsection{SITUACION GENERAL}

En la actualidad hay fabricantes que promocionan la venta de coches autónomos en circulación, cuando no lo son. Se mencionaba en el BI Intelligence [8], que en el 2020 habría 10 millones de vehículos autónomos, pronóstico fallido a todas luces. Lo primero que se verá como vehículo autónomo, en nivel 4, serán los denominados "robotaxis" [9]; serán muy distintos de los vehículos autónomos privados y con normativa legal específica. Estos taxis autónomos, que desarrollan marcas como Weymo (Google) y Uber, ofrecerán servicios denominados como "Share Mobility" [10] en los que el cliente comparte gran porcentaje de los costes y se moverán por entornos muy controlados, cerrados y sin salir en modo autónomo. El nivel 4 para el conductor particular llegará mucho mas tarde, por razones de sobre coste.

Los sistemas sensores y de procesamiento están actualmente en modo prototipo. El servicio de los robotaxis será intensivo y continuo, 24 horas, 365 días, para una amortización rápida.

Además, los sistemas sensores actuales tienen un tamaño y peso no integrable en el diseño de vehículos, lo cual provocaría un empeoramiento de las cualidades aerodinámicas y el consecuente incremento del consumo energético. Otro inconveniente es lo relativo al elevado coste de mantenimiento de estos sensores. Mientras que Weymo y Uber envían el dispositivo a reparar o directamente lo sustituyen, el usuario privado no se puede permitir esta operación y, menos aun, inmovilizar el vehículo por largos periodos. Este hardware no está en la actualidad preparado para el apoyo integral de la conducción autónoma. Merece la pena mencionar, a modo de ejemplo, que el laser3D del futuro, que dará soporte a la conducción autónoma, no deberá tener parte móviles, ser más eficiente y ofrecer mayor sensibilidad y precisión.

Se presentan dos desafíos, uno enorme tecnológicamente y otro legalmente. Se publicaba recientemente un listado de fabricantes Estadounidenses [11] que tienen vehículos aprobados legalmente para circular por carretera y se verificó que solo era en California. En esta lista aparece General Motors liderando el ranking, que compró en 2016 la startup Cruise Automation.

Hablando ahora de las empresas que lideran, en inversión y potencialidad, el desarrollo de vehículos autónomos, Weymo (Google) sobrepasa con diferencia a las demás; a continuación se sitúa Uber, a notable distancia. Aparecen también las compañías General Motors, Ford y Tesla, seguida de los fabricantes alemanes, BMW, Mercedes y Audi: por último empresas como Nissan, Toyota, Volvo y Hyundai. A parte de las grandes compañías, emergen startups anónimas, Comma.ai (George Hotz) [12], que se atreven en la automatización del vehículo.

Waymo es la que dispone de la mayor flota de vehículos; sus coches han recorrido más de 8 billones de kilómetros [13]. BMW indica en sus comunicados oficiales, que en 2021 sacará al mercado la versión iNEXT, un modelo con nivel automatizado por encima de nivel 3. Tesla anuncia bastante más de lo que tecnológicamente tiene, es decir, realmente sus vehículos no alcanzan el nivel 3, con la sensorización que dispone su nuevo Modelo 3.

El nuevo Audi A8, la versión limusina, probablemente sea a día de hoy, el vehículo más avanzado tecnológicamente en el mercado. El Audi A8 cuando se presentó, se lanzó como el primer automóvil del mercado que tenía funcionalidades de conducción autónoma a nivel 3, es decir, que 
en determinadas circunstancias durante unos segundos, especialmente el modo autopista y modo atasco, el vehículo es autónomo. La realidad es que no lleva estas funciones activas y la explicación oficial de la marca es que no existe normativa para automóvil con un nivel 3 de conducción autónoma.

En los últimos cinco años podemos decir que ha habido un boom y una burbuja de la conducción autónoma, que ha difundido la creencia de que la conducción completamente autónoma estaba muy cerca, cuando en realidad no era así. En los últimos meses prácticamente ya no hay noticias, o las noticias que hay se han vuelto negativas, debido a los diversos accidentes del vehículo de Uber como de los coches de Tesla, creando bastante alarma social, desconfianza y percepción contradictoria.

La conducción autónoma es un negocio tan fabuloso que muchas empresas quieren estar en la vanguardia e incluso algunas necesitan la conducción autónoma para hacer más rentable su negocio como pueden ser las empresas propietarias de taxis.

Ya es hora de que los diferentes centros neurálgicos de la industria del automóvil (Europa, Japón y EEUU) se pongan de acuerdo y redacten una legislación común general. La normativa actual deriva de la convención de Viena, del año 68 , justamente hace 50 años, lo que ha provocado que las diferentes legislaciones nacionales en materia de tráfico estén completamente obsoletas si pensamos en términos de conducción autónoma.

\subsubsection{SITUACION PARTICULAR DE TESLA}

Del fabricante Estadounidense Tesla, se puede exponer que anuncia un producto que excede a los avances reales ofrecidos, respecto a la conducción autónoma completa. Lo que ofrece actualmente en sus vehículos es un nivel 2, bien desarrollado en autopista, con las limitaciones propias de nivel 2, más aún disponiendo solamente sensores de ultrasonidos, radar y cámaras trifocales de video. Para alcanzar un nivel 4 ó 5, se necesita una redundancia de sensores, que no instala Tesla en sus vehículos. El CEO Tesla Elon Musk está en contra del uso de LIDAR 3D, por el inconveniente de su elevado coste. Además, la plataforma de procesado de los datos capturados por sus sensores no es redundante. El Full Autonomous Drive System que ofrecen, solo puede alcanzar un nivel 3, limitado a baja velocidad. Esta política comercial de posicionarse como la marca puntera en conducción autónoma, provoca en EEUU, que miles de clientes compren un vehículo Tesla pensando que su coche conduciría solo.

\subsubsection{INTELIGENCIA ARTIFICIAL Y REDES NEURONALES}

Está palabras definen una serie de algoritmos que operan en un hardware específico para obtener buen rendimiento, y que en base a un aprendizaje previo, con una serie de clasificadores, extraen los parámetros importantes y proporcionan una información a un sistema de toma de decisiones. En la actualidad presentan el problema del desconocimiento de técnicas precisas para su validación efectiva, es decir, no existe humano que valide un sistema formado únicamente por algoritmos de inteligencia artificial. Lo deseado en un sistema controlado por un algoritmo, es un comportamiento esperado A ante una entrada determinada B, sin sorpresas. La inteligencia artificial a día de hoy si es válido para ciertas aplicaciones específicas, por ejemplo, predicción de posibles rutas de conducción, que pueden ser validadas, pero en general, se desconoce cuándo va a fallar un algoritmo ante unas condiciones de contorno tan cambiantes. Se estima que se necesitan 50 millones de kilómetros recorridos para validar un sistema de conducción autónoma [14]. Para producir en serie estos sistemas de inteligencia artificial implementados en un vehículo, aun está muy lejos. 


\subsection{FUTURO}

Para una integración total del vehículo autónomo en la sociedad moderna, hay que reinventar ciudades, carreteras, infraestructuras, etc,. Supone también cambiar el modo o la utilización de la conducción. En un futuro de conducción autónoma, con energía renovable integrada y tecnología compartida, el vehículo autónomo será un servicio antes que una propiedad. El vehículo autónomo no tiene sentido estar aparcado en la puerta de casa o en nuestro parking privado, debido a que vendrá a recogernos y se usará cierto tiempo. Durante el siglo XX, la cultura humana ha estado pagando por un consumible como es el coche, que solo se utiliza durante el $5 \%$ del día [15], este concepto de consumo y propiedad también se extinguirá, es decir, se pagará por los minutos de uso de un vehículo genérico autónomo, como se hace con la aplicación "Carshare".

La fisionomía de las ciudades cambiará sin duda. Por poner un ejemplo, la gestión del aparcamiento [22] mejorará mucho y con ello la mejora en la fluidez de la circulación. Por ejemplo, en un aparcamiento para vehículos autónomos caben muchos más, porque no hacen falta espacios para poder abrir las puertas. Con el aumento de la población, la cantidad de vehículos de 4 ruedas también están aumentando, lo que incrementa los problemas en la mayoría de las ciudades metropolitanas. El problema es más agudo en los países en desarrollo. Los aparcamientos en la calle reduce la velocidad del flujo y crea congestión. Esta conlleva la pérdida de tiempo y costo. El espacio insuficiente en el área urbana, conlleva menos estacionamiento, y a su vez, obligan a los usuarios a aparcar sus vehículos en la calle. Una política adecuada de gestión del estacionamiento debe ser enmarcado para cada área urbana para resolver problema de aparcamiento.

También y la organización del tiempo cambiarán considerablemente, cuando los trayectos se realicen con vehículos autónomos. Está en plena expansión el negocio de los datos para entretenimiento, el negocio de la atención humana y la industria del automóvil está explorando este campo, en esta sociedad que valora el poder de captación de la atención humana. Será muy importante conocer las tendencias de tránsito de una ciudad, para determinar donde están las necesidades.

Otro aspecto que cambiará considerablemente en el futuro será el funcionamiento de las compañías aseguradoras. Surgen preguntas clave, por ejemplo, ¿quién es responsable ante el atropello de un peatón por un vehículo autónomo?, el fabricante del software, el pasajero (que no será ya conductor) o la infraestructura de la ciudad?. Los riesgos de accidente se medirán de otro modo, las compañías aseguradoras, a través de los datos recogidos por los vehículos autónomos, tendrán muchos más datos de los que disponen hoy en día. Conocerán como conducimos, a qué horas y frecuencia para establecer las cuotas a pagar

Las diferentes administraciones se ven afectados desde varios puntos de vista:

- si el vehículo autónomo cumple con las normas de circulación y seguridad, no recaudarán por las infracciones.

- al no haber accidentes supone un gran ahorro en prestaciones sanitarias.

- del mismo nivel que se ahorra por el decrecimiento de contaminación.

- aumento de espacio disponible por la eliminación de aparcamientos en el centro de ciudades.

Los reguladores normativos tendrán que reinventar toda la leyes legales de circulación, seguridad y el gran problema de la ética moral, resolver el dilema del tranvía [16]. Los vehículos autónomos podrán tomar decisiones morales y éticas como lo hacen los humanos [17], decisiones de vida o muerte. El dilema de elegir, ante dos situaciones adversas, cual es éticamente la menos mala. 
Por ejemplo, elegir la decisión de auto-estrellarse ante la inminente atropello de un peatón, con el consiguiente peligro de matar al propio conductor [18], según muestra la Figura 3.
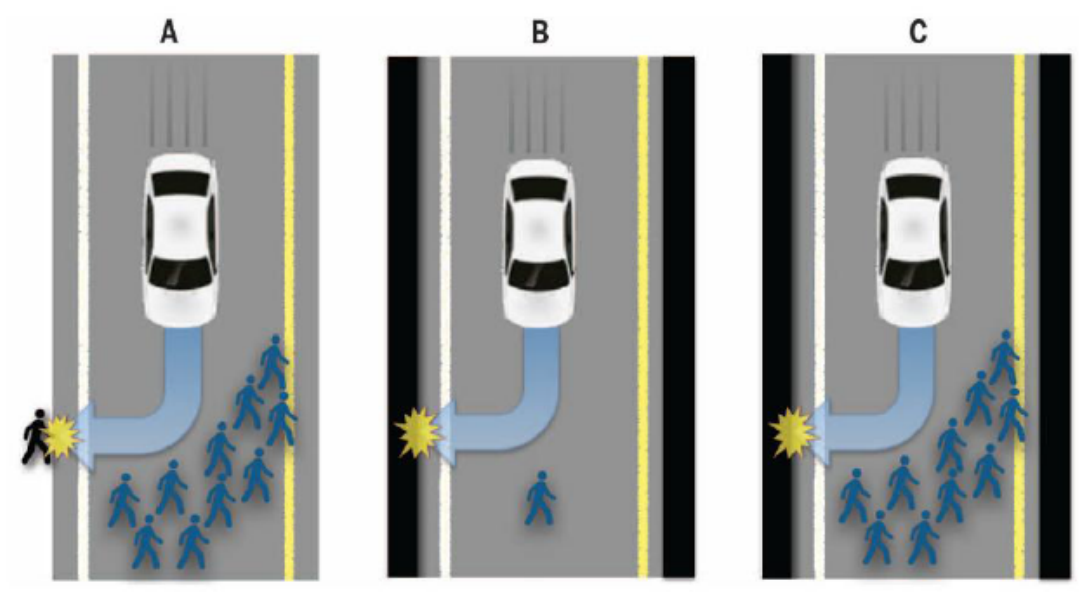

Figura 3. Toma de decisiones morales y éticas propias de los VAS
A, atropellar varios peatones o un solo peatón
B, atropellar un solo peatón o herir al conductor
C, atropellar varios peatones o herir al conductor

El Instituto de Tecnología de Massachusetts (MIT) ha realizado estudios en este sentido [19], desafían el supuesto de que las decisiones morales dependen del contexto y no pueden ser modeladas o descritas algorítmicamente [20]. Las investigaciones sugieren, que el comportamiento moral humano, puede ser caracterizado por algoritmos y utilizado por las máquinas para resolver dilemas morales en situaciones reales. Estas investigaciones demostraron que las decisiones morales en el ámbito de las colisiones de tráfico inevitables pueden ser explicadas bien, y modeladas, por un solo valor de la vida para cada objeto humano, animal o inanimado. Según Mercedes, la tendencia es primero proteger al conductor y a sus acompañantes.

El profesor Gordon Pipa [21], del Instituto de Ciencias Cognitivas de la Universidad de Osnabrück, Alemania, dice que dado que ahora parece posible que las máquinas puedan ser programadas para tomar decisiones morales humanas, es crucial que la sociedad emprenda un debate serio y urgente, "necesitamos preguntar si los sistemas autónomos deben adoptar juicios morales, en caso afirmativo, si imitan la conducta moral imitando las decisiones humanas, si se comportan a lo largo de las teorías éticas y, en caso afirmativo, cuáles y críticamente, si las cosas van mal ¿quién o qué?.

Como ejemplo, dentro de los nuevos principios éticos alemanes, un niño que corre hacia la carretera se clasificaría como implicado de manera significativa en la creación del riesgo, por lo tanto menos calificado para ser salvado en comparación con un adulto parado en el sendero como parte no involucrada. Pero ¿es esto un valor moral sostenido por la mayoría de la gente?. 
El proyecto CityMobil2 [23] de Europa está demostrando aplicaciones de tránsito de baja velocidad y totalmente autónomas en cinco ciudades. Además, los vehículos autónomos se están volviendo común en otros sectores, incluyendo el militar, la minería y la agricultura. Estamos en la cúspide de una nueva era en la movilidad, dado que las tecnologías como vehículos autónomos están casi listos para su despliegue y pruebas [24]. Se están cruzando las fronteras tecnológicas para este despliegue, sabemos el impacto potencial de tales tecnologías sobre la forma urbana y los patrones de uso de las carreteras. Se trata de abordar estas cuestiones simulando el funcionamiento de vehículos autónomos compartidos (SAV) [25], utilizando redes de transporte real con el nivel de enlace calibrado de viaje, velocidades y matriz de origen-destino. Los resultados del modelo sugieren que el sistema SAV puede reducir las parcelas de estacionamiento en un 4,5\%, con un nivel de penetración del mercado del 5\%. En escenarios de estacionamiento saturado, será posible derivar el mismo a áreas adyacentes de forma automática.

Existirán infraestructuras conviviendo en paralelo, infraestructuras de vehículos conducido por humanos y nuevas infraestructuras diseñadas desde cero, para vehículos autónomos. Ya se realizan pruebas reales de esta tecnología en campus universitarios y parques empresariales de gestión privada [26]. En este entorno se introduce los prototipos de taxis voladores autónomos que Airbus ha presentado recientemente, que pueden llevar hasta cuatro pasajeros [27]. 
3 CAPITULO 3

\section{ROBOTIZACION DE CLOUD INCUBATOR}

CAR (CICAR)

\subsection{INTRODUCCION}

La tarea de conducir un vehículo es, para la mayor parte de conductores, una actividad de bajo riesgo, requiere una serie de habilidades, que pueden ser desarrolladas en un corto período de tiempo. Sin embargo, la información que el usuario debe procesar excede sus capacidades. Según estudios realizados sobre datos de accidentes, más del $90 \%$ de ellos tienen el factor humano como una de sus causas [28].

Por esta razón, los vehículos inteligentes están destinados a ayudar al conductor, reduciendo su carga, aumentando y mejorando la información disponible para promover una conducción segura y eficiente sin errores, y lograr una mejor respuesta.

En el Laboratorio de Visión Artificial y Vehículos Inteligentes (VAVI) de la Universidad Politécnica de Cartagena, hemos desarrollado la plataforma para el desarrollo de tecnología de conducción autónoma. Esta plataforma permite una experimentación completa en los aspectos relacionados con la integración de sensores (LIDAR 3D, LIDAR 2D, sistemas de posicionamiento global, cámaras de visión, etc.), control de dispositivos (motores, actuadores, etc.) o la integración de sistemas industriales para la comunicación y puesta en común de la información entre vehículos autónomos vehículo a vehículo (V2V) y entre vehículo a infraestructura vial (V2I) que mejora la seguridad.

\subsection{IMPLEMENTACION MECANICA CLOUD INCUBATOR CAR (CICAR)}

La plataforma CICar elegida, se basa en un vehículo comercial eléctrico Renault Twizy, Figura 4. Su elección tuvo los factores determinantes siguientes:

- Bajo coste $<6.000 €$.

- Eléctrico $100 \%$ con una mecánica sencilla y bajo mantenimiento.

- Pocas unidades de control electrónicas (ECUs) que han permitido la manipulación de los elementos de control del vehículo con facilidad.

- Fácil acceso a los planos eléctricos y mecánicos para su correcta manipulación. 
Ha sido convenientemente modificado para cumplir con las especificaciones mencionadas anteriormente. Dichas modificaciones se consideran como la arquitectura de nivel bajo e incluyen:

a) la automatización de los elementos de conducción (volante, freno, acelerador, sentido de la marcha);

b) instalación de anclajes externos capaces de contener una amplia variedad de sensores;

c) instalación de bastidores interiores para acomodar los diferentes sistemas de control y procesamiento;

d) instalaciones eléctricas y buses de comunicación para alimentar y conectar los diferentes sensores y hardware de control y procesamiento.

Para la conducción autónoma del mismo es preciso robotizar los sistemas básicos de conducción que son volante, freno, acelerador y sentido de la marcha.

La arquitectura ha sido reformada, incrementando su modularidad, permitiendo el montaje de nuevos sensores.

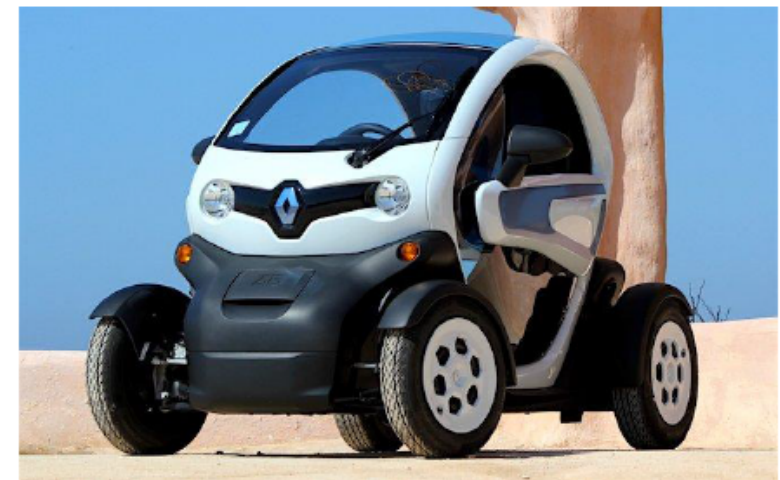

Figura 4. Vehículo comercial, Twizy

\subsubsection{ROBOTIZACION DEL SISTEMA DE DIRECCION}

El objetivo de la robotización del sistema volante es el control automatizado de la dirección y giro del vehículo.

Para ello se ha propuesto una solución mecánica básica, basada en actuar sobre la columna o eje de dirección, Figura 5 y montando en paralelo un conjunto mecánico moto-reductor, con engranajes, que hace rotar el eje de volante, de modo directo.

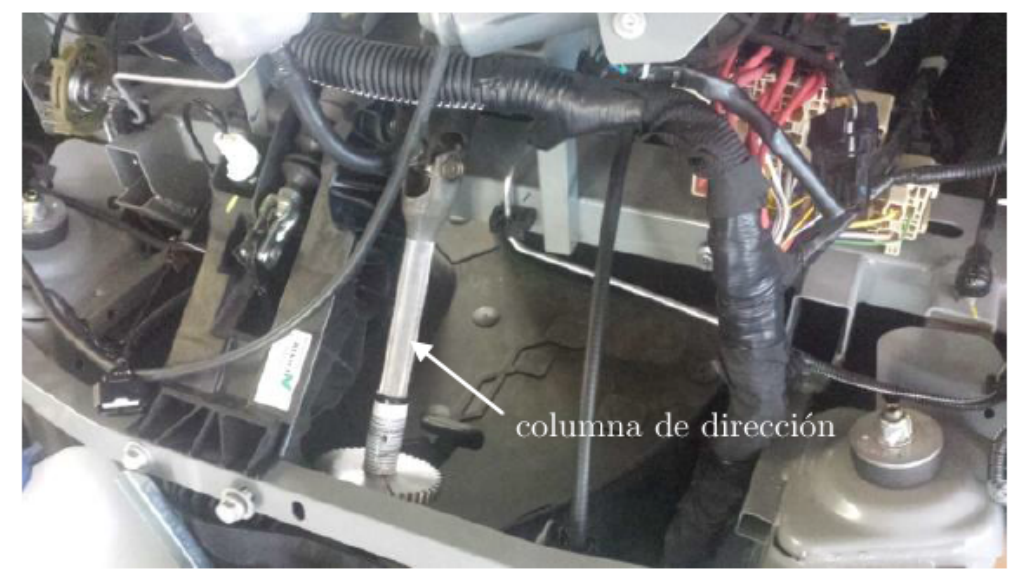

Figura 5. Sistema volante en estado original 
El resultado de esta solución mecánica propuesta ha permitido obtener controlado los movimientos giratorios del vehículo para lograr alcanzar posiciones de objetivo.

No es necesario desmontar el eje, la solución adoptada es instalar un engranaje conducido partido, que abraza el árbol de dirección. En la Figura 6 se muestra el diseño 3D por ordenador empleado para la construcción.

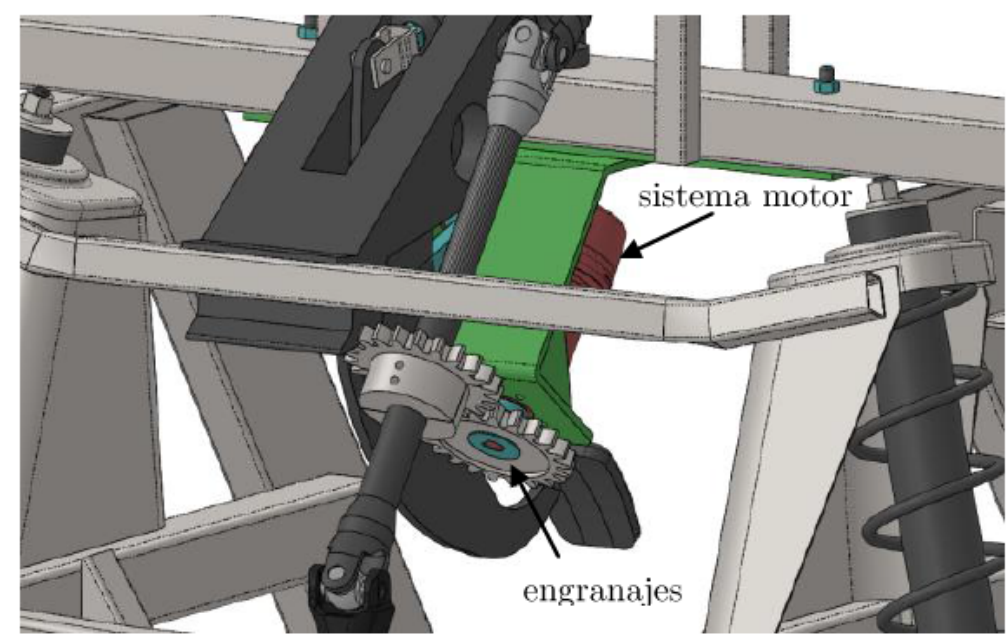

Figura 6. Diseño 3D de la solución adoptada para volante

Tras varios estudios de la implementación, buscando la zona óptima para el montaje del sistema, se determinó su ubicación paralela al eje de dirección como solución final a desarrollar, Figura 7 .

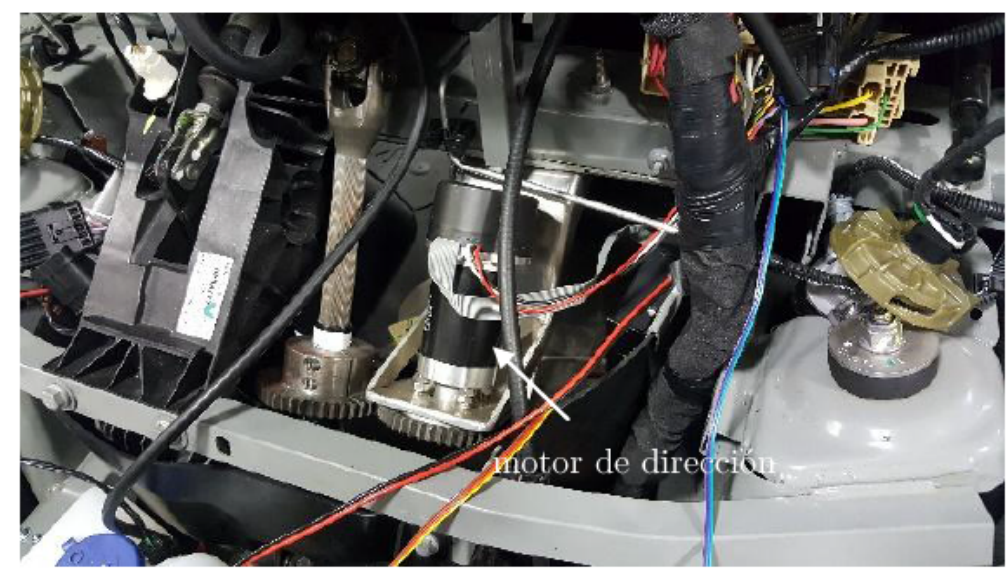

Figura 7. Imagen del sistema automatizado de volante

La implementación del control de posición y velocidad debe ejecutarse estimando los parámetros a los que está sujeto el sistema. La conducción humana, en condiciones normales, hace girar el volante a una velocidad entre unos 180 grados/segundo (30 rpm) y 360 grados/segundo (60 rpm), ejerciendo una fuerza tangencial de unos $20 \mathrm{~N}$. 
Los parámetros iniciales para selección de moto-reductor y diseño mecánico de engranajes del volante, Figura 8 son:

-Fuerza de rotación $\mathrm{F}=20 \mathrm{~N}$

-Par de rotación $\mathrm{M}=0,30 \mathrm{Nm}$

-Velocidad máxima de rotación $\omega 002 \pi \mathrm{rad} / \mathrm{s}(60 \mathrm{rpm})$

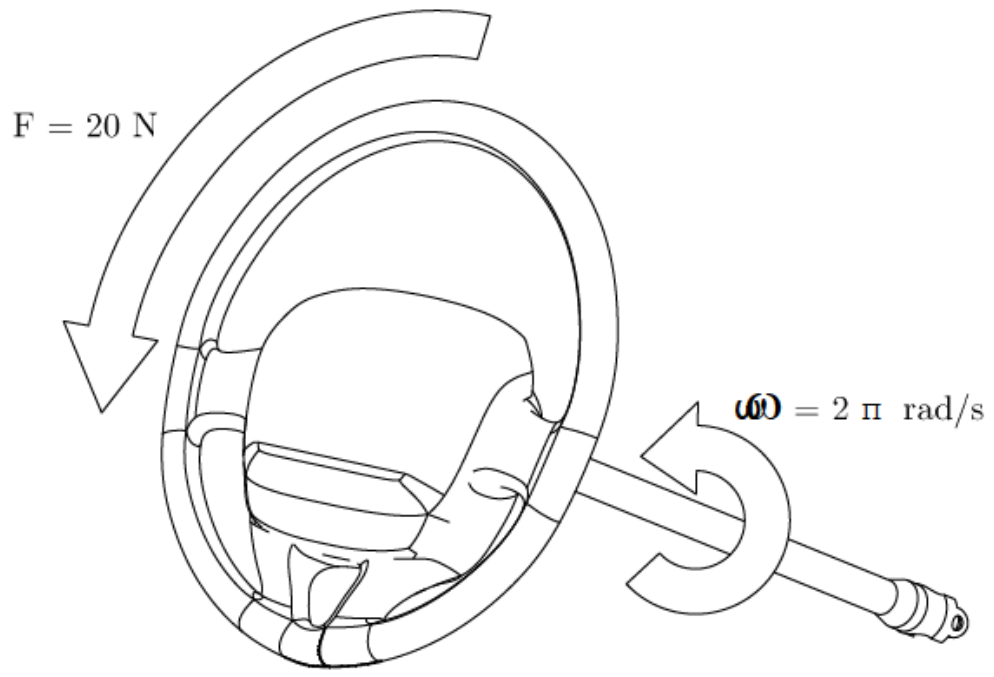

Figura 8. Parámetros iniciales de cálculo para volante

La potencia necesaria para mover el volante a la velocidad angular indicada resulta ser:

$$
\begin{aligned}
& P_{1}=\frac{d W}{d t}=F \cdot D \cdot \frac{d \theta}{d t}=M \cdot \frac{d \theta}{d t}=M \cdot \omega \\
& P_{1}=20 \mathrm{~N} \cdot 0,15 \mathrm{~m} \cdot 6,3 \frac{\mathrm{rad}}{\mathrm{s}}=18,90 \mathrm{w}
\end{aligned}
$$

Considerando las pérdidas por transmisión de engranes $\eta_{1}$, rozamiento de ruedas con el asfalto $\eta_{2}$, y rendimientos mecánicos $\eta_{3}, \quad 0,60 \quad 0,60$ y 0,70 respectivamente valores según consideraciones norma ANSI/AGMA 2101-D04:

$$
P_{2}=\frac{P_{1}}{\eta_{1} \cdot \eta_{2} \cdot \eta_{3}}=\frac{18,90 \mathrm{w}}{0,60 \cdot 0,60 \cdot 0,70}=75,50 \mathrm{w}
$$

Se ha seleccionado un motor de potencia estándar 100w con una velocidad nominal angular de salida de $3.260 \mathrm{rpm}$ y con un par nominal 0,231 Nm. 
Para el diseño de los engranajes de transmisión, se parte de una configuración como muestra la Figura 9.

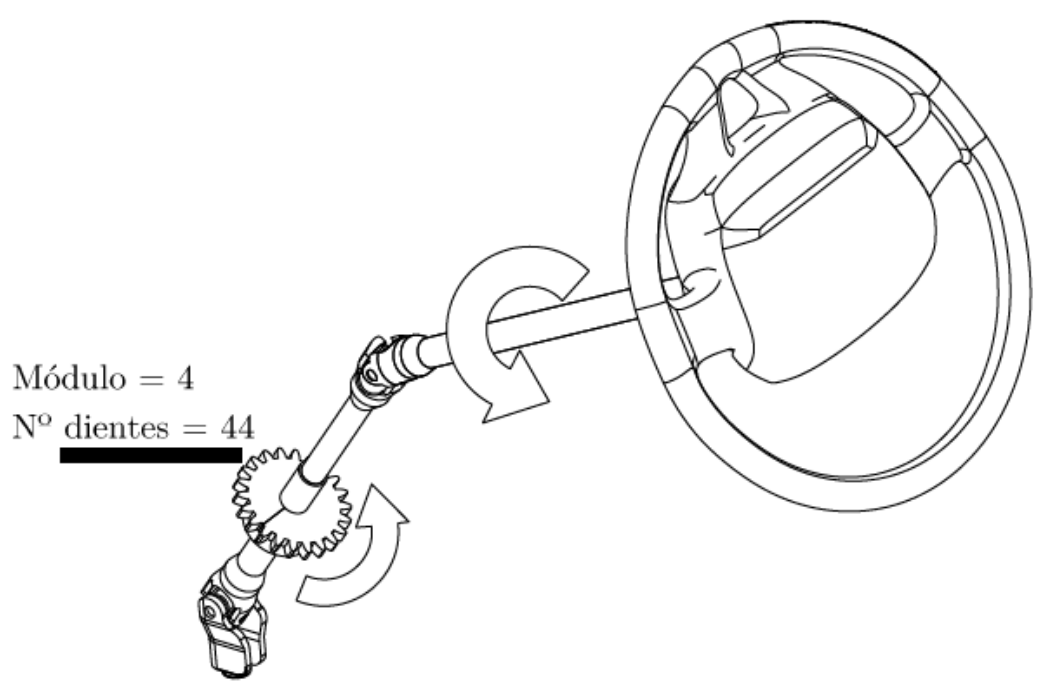

Figura 9. Diseño 3D para diseño de engranajes de volante

Por tanto la relación de reductor necesaria es:

$$
r=\frac{3.260 \mathrm{rpm}}{60 \mathrm{rpm}}=54,33
$$

El reductor con la relación más cercana tiene relación de transmisión 1:53.

Con el moto-reductor seleccionado, las condiciones de salida reales son mostradas en la Figura 10:

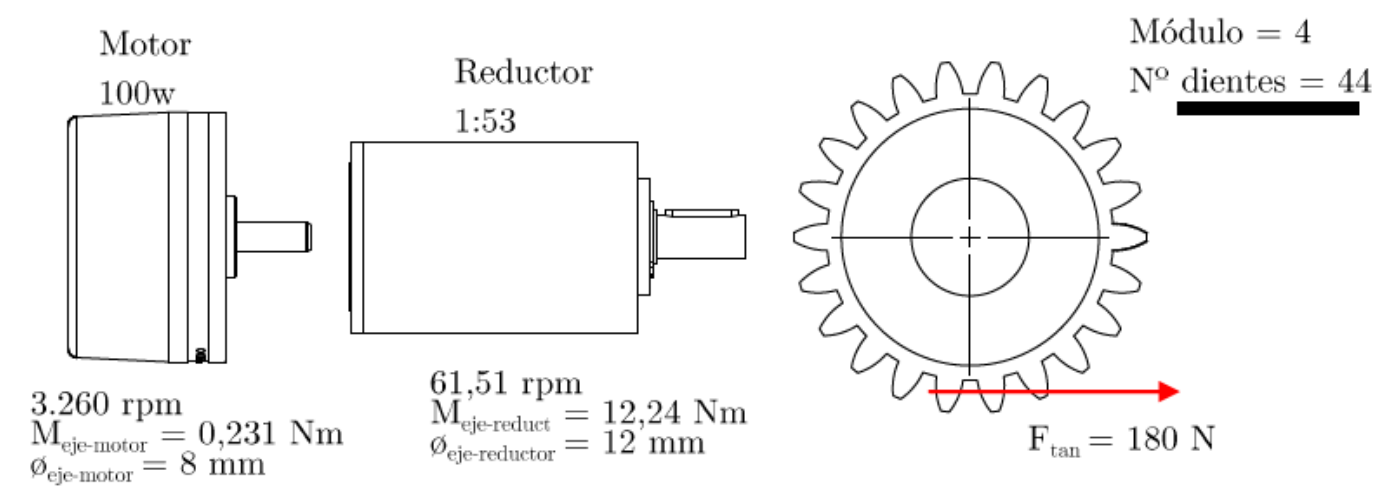

Figura 10. Parámetros de cálculo moto-reductor volante

La fuerza estimada de empuje tangencial es:

$$
F_{\text {tang }}=\frac{P}{r_{\text {engranaje }} \cdot \omega}=\frac{100 \mathrm{w}}{\left(\frac{0,176}{2}\right) \mathrm{m} \cdot 6,3 \mathrm{rad} / \mathrm{s}}=180 \mathrm{~N}
$$




\subsubsection{ROBOTIZACION DEL SISTEMA DE FRENADO}

El objetivo de la robotización del sistema freno es el control automatizado de la frenada y detención del vehículo.

Para ello se ha propuesto una solución mecánica básica, basada en actuar directamente empujando el pedal, a través de otro conjunto mecánico moto-reductor con una leva, Figura 11. La solución adoptada permite tener en todo momento el pedal disponible para la actuación manual en caso de emergencia, la robotización no anula el movimiento de fábrica.

El resultado de esta solución mecánica propuesta ha permitido obtener controlado el tiempo de frenado del vehículo para lograr alcanzar posiciones de objetivo.

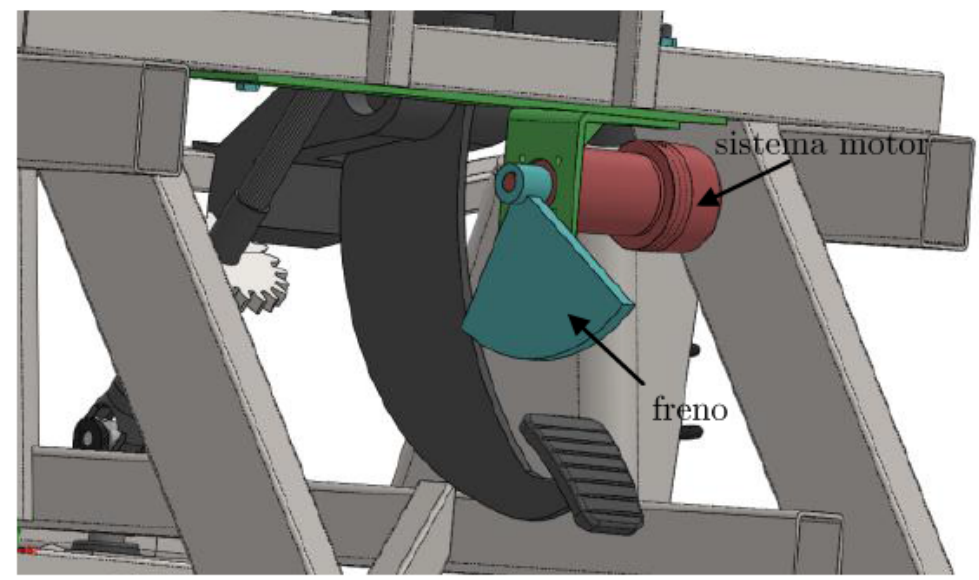

Figura 11. Diseño 3D de la solución adoptada para el freno

La actuación humana sobre el pedal de freno, en una situación normal de frenada controlada, ejerce una fuerza de $150 \mathrm{~N}$ [29]. El recorrido total del pedal es de unos $15^{\circ}$ aproximadamente, que se realiza en una décima de segundo.

Los parámetros iniciales para selección de moto-reductor y diseño mecánico del freno, Figura 12 son:

-Fuerza de empuje $\mathrm{F}=150 \mathrm{~N}$

-Par de rotación $\mathrm{M}=30 \mathrm{Nm}$

-Velocidad máxima de rotación $\omega 00,84 \Pi \mathrm{rad} / \mathrm{s}(25 \mathrm{rpm})$

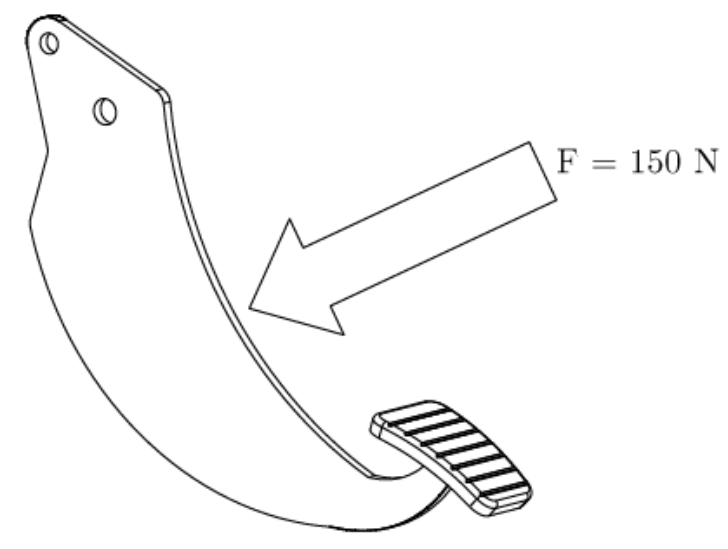

Figura 12. Parámetros iniciales de cálculo para freno 
La potencia mínima necesaria para mover el pedal de freno a la velocidad angular indicada resulta ser:

$$
\begin{gathered}
P_{1}=\frac{d W}{d t}=F \cdot D \cdot \frac{d \theta}{d t}=M \cdot \frac{d \theta}{d t}=M \cdot \omega \\
P_{1}=150 \mathrm{~N} \cdot 0,20 \mathrm{~m} \cdot 2,64 \frac{\mathrm{rad}}{\mathrm{s}}=79,20 \mathrm{w}
\end{gathered}
$$

Considerando las pérdidas por transmisión de engranes $m_{1} \mathrm{y}$ rendimiento mecánicos $\mathbf{m}_{2}$, 0,97 y 0,98 respectivamente valores según consideraciones norma ANSI/AGMA 2101-D04:

$$
P_{2}=\frac{P_{1}}{\eta_{1} \cdot \eta_{2}}=\frac{79,20 w}{0,97 \cdot 0,98}=83,37 w
$$

Se ha seleccionado el mismo motor que el volante de potencia estándar 100w con una velocidad nominal angular de salida de $3.260 \mathrm{rpm}$ y con un par nominal 0,231 Nm. El montaje real se muestra en la figura 16 .

La relación de reductor necesaria es:

$$
r=\frac{3.260 \mathrm{rpm}}{25 \mathrm{rpm}}=130,40
$$

El reductor con la relación más cercana tiene relación de transmisión 1:156.

Con el moto-reductor seleccionado, las condiciones de salida reales son mostradas en la Figura 13:
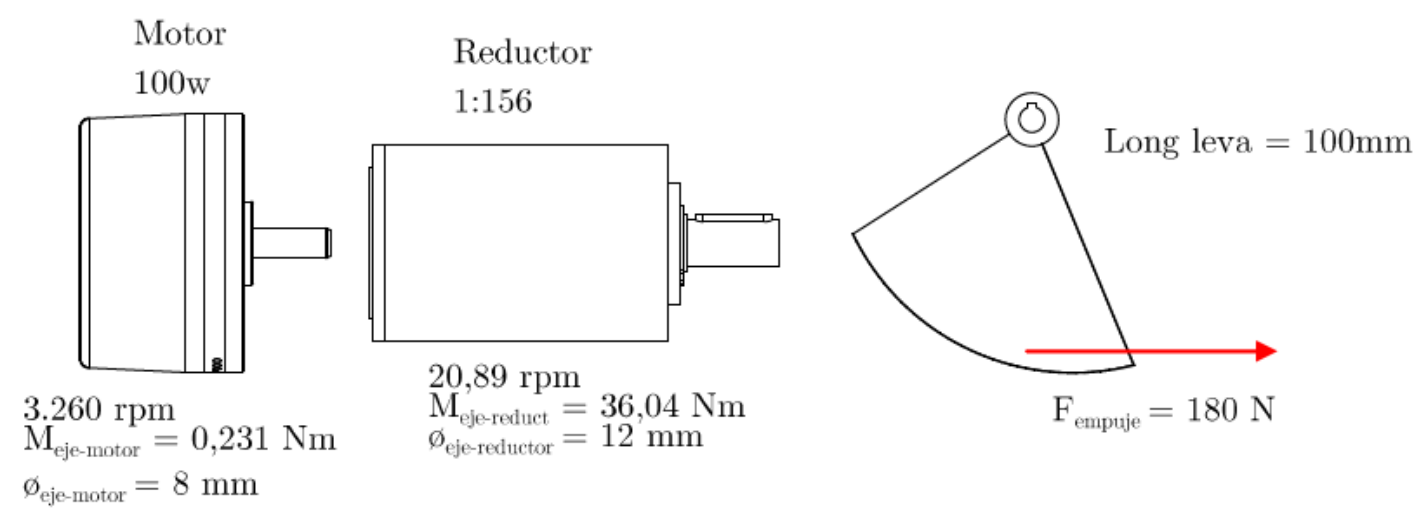

Figura 13. Parámetros de cálculo moto-reductor freno

La fuerza estimada de empuje es:

$$
F_{\text {empuje }}=\frac{P}{r_{\text {leva }} \cdot \omega}=\frac{100 \mathrm{w}}{\left(\frac{0,100}{2}\right) \mathrm{m} \cdot 2,62 \mathrm{rad} / \mathrm{s}}=763 \mathrm{~N}
$$




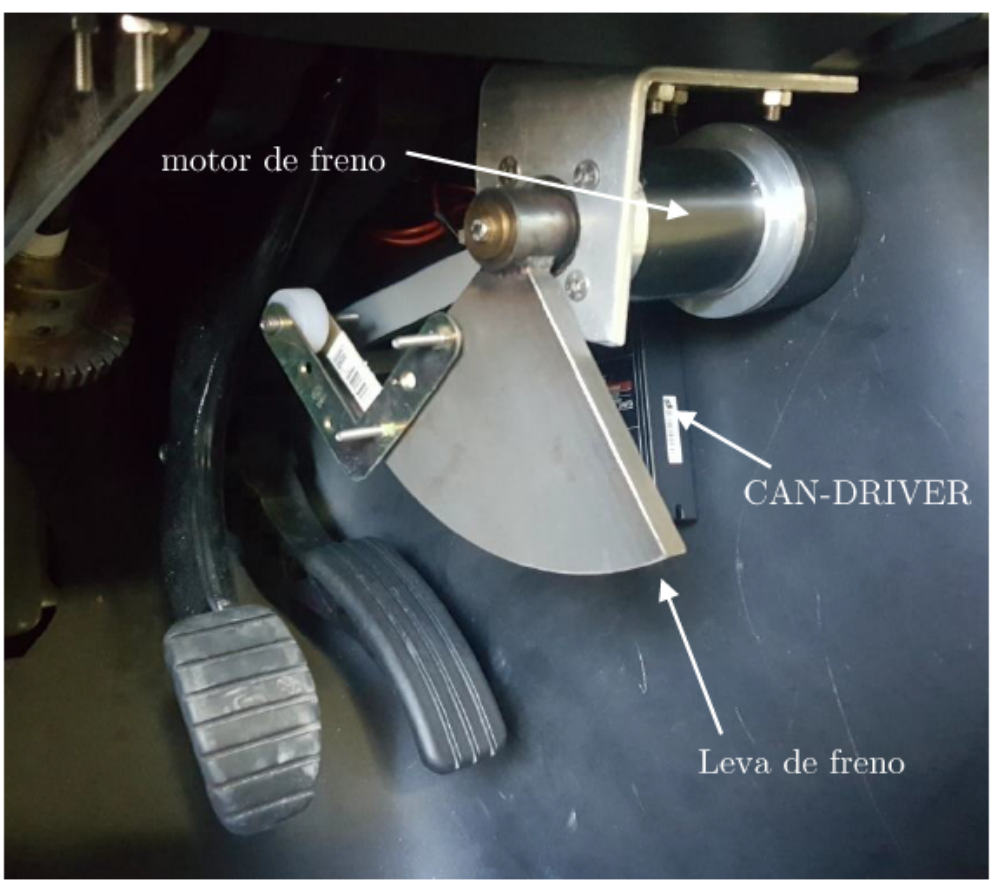

Figura 14. Imagen del sistema automatizado de freno

\subsubsection{ROBOTIZACION DEL SISTEMA DE ACELERACION $\quad \mathrm{Y}$ SELECCION DEL SENTIDO DE MARCHA}

El objetivo de la robotización del sistema acelerador es el control automatizado de aceleración del vehículo.

Para ello se ha propuesto una solución electrónica básica, previamente se ha muestreado los niveles de tensión de funcionamiento del sistema electrónico, Figura 15. A través de arquitectura informática de la unidad de control cRIO se controla dichos niveles de tensión y es posible la aceleración.

El resultado de esta solución propuesta, en este caso electrónica, ha permitido obtener controlado el acelerador del vehículo para lograr alcanzar posiciones de objetivo.

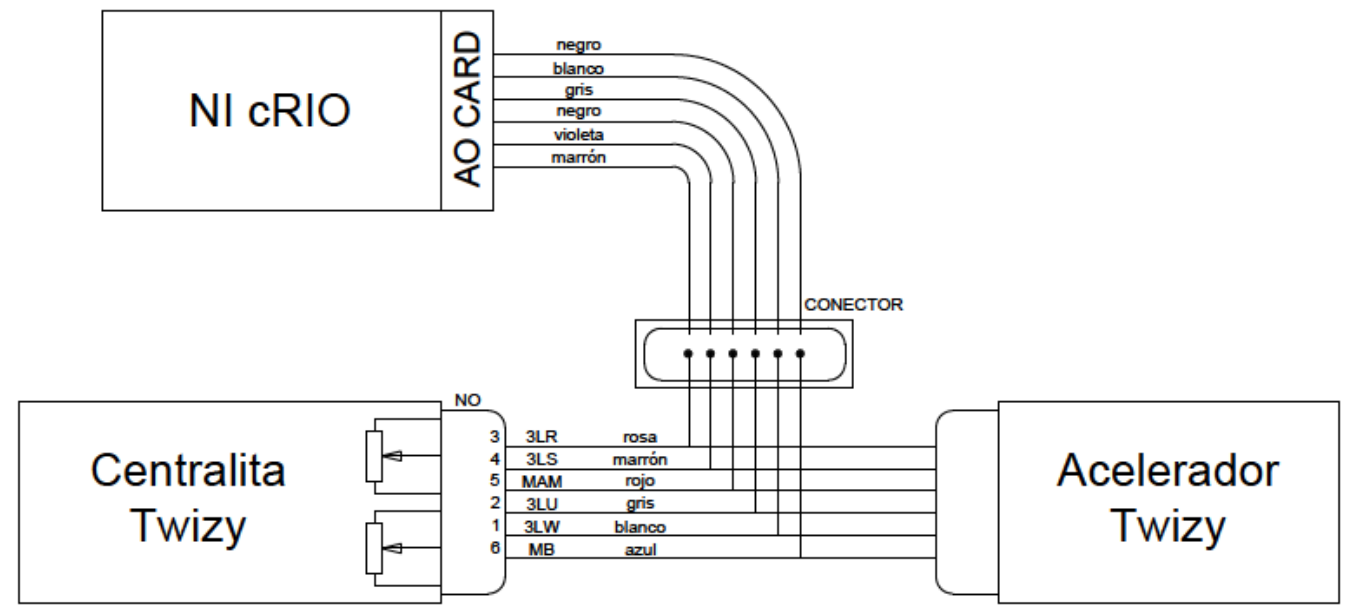

Figura 15. Esquema modificación conexión acelerador 
Las señales analógicas de tensión se generan con un módulo salidas de voltaje de la Serie C NI9264 $25 \mathrm{kS} / \mathrm{s} /$ canal Simultáneo, $\pm 10 \mathrm{~V}, 16$ Canales.

Los valores muestreados en las dos posiciones extremas del acelerador, se tomaron con una tarjeta AI de cuatro canales, Figura 16.

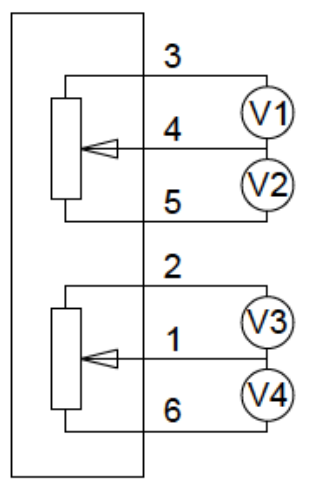

Figura 16. Esquema captura datos niveles de tensión

y arrojaron los siguientes resultados, mostrados en la Tabla 1.

\begin{tabular}{ccccc}
\hline $\begin{array}{c}\text { Posición } \\
\text { acelerador }\end{array}$ & V1 & V2 & V3 & V4 \\
\hline $\min$ & $6,94 \mathrm{v}$ & $1,81 \mathrm{v}$ & $9,18 \mathrm{~V}$ & $1,04 \mathrm{~V}$ \\
$\max$ & $0,69 \mathrm{v}$ & $8,00 \mathrm{~V}$ & $5,57 \mathrm{v}$ & $4,64 \mathrm{~V}$ \\
pendiente & $m_{V 1}=\frac{6,94-0,69}{10-0}=0,62$ & $m_{V 2}=\frac{1,81-8,00}{10-0}=-0,62$ & $m_{V 3}=\frac{9,18-5,57}{10-0}=0,36$ & $m_{v 4}=\frac{4,64-1,04}{10-0}=0,36$ \\
recta & $y_{V 1}=0,62 \cdot x+6,94$ & $y_{V 2}=-0,62 \cdot x+1,81$ & $y_{V 3}=0,36 \cdot x+9,18$ & $y_{V 4}=0,36 \cdot x+1,04$ \\
pendiente &
\end{tabular}

Tabla 1. Valores muestreados de tensión acelerador

Con estos datos se obtienen las ecuaciones lineales necesarias para el control electrónico.

Para la robotización del sentido de marcha, es necesario actuar electrónicamente sobre los pulsadores de dirección de marcha. El vehículo dispone de tres botones de sentido de marcha, hacia adelante (D), hacia atrás (R) y punto neutro o muerto $(\mathrm{N})$, figura 19. Las señales digitales se generan con un módulo digital de la serie C NI9485, 8 Canales, Relé SSR, 60 VDC/30 Vrms, 750 $\mathrm{mA}$.

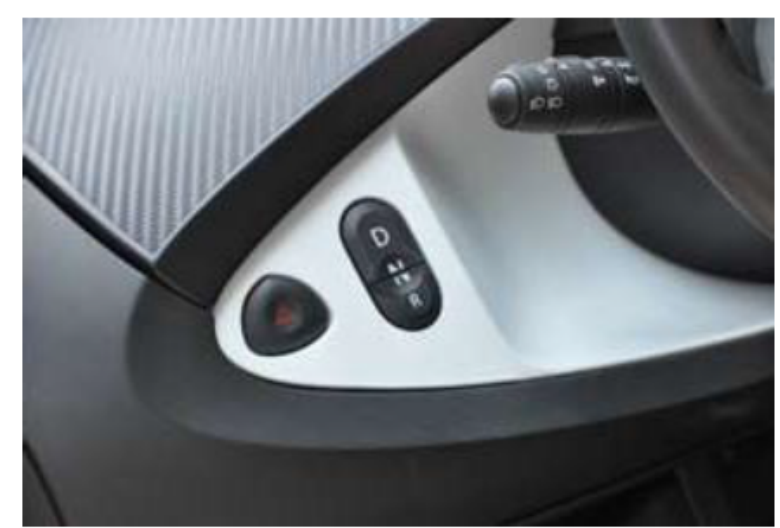

Figura 17. Pulsadores sentido de marcha 


\subsection{CONTROL TRAYECTORIAS. CONFIGURACIÓN}

\section{ACKERMAN}

\subsubsection{ARQUITECTURA ACKERMAN}

La arquitectura Ackerman se aplica en la actualidad y universalmente en todos los coches. Fue inventado por el constructor de transporte alemán "Lankensperger" en 1817, luego de ser patentado por su agente en Inglaterra Rudolph Ackermann (1764-1834) en 1818 para los coches de caballos. Es el sencillo mecanismo que realiza la unión entre los ejes de las ruedas directrices del vehículo con el fin de que pueda producirse un cambio de dirección sin que exista deslizamiento de las ruedas sobre el suelo. Para ello es necesario que los ejes de todas las ruedas pasen por un mismo punto. El cuadrilátero de Ackerman permite que esta condición se satisfaga con una aproximación bastante buena.

\subsubsection{IMPLEMENTACION EN EL CICAR}

Toma importancia en este capítulo la formulación e implementación para controlar la dirección de las ruedas delanteras y rotación de las ruedas traseras motrices para realizar trayectorias de movimiento. La configuración típica de los automóviles de cuatro ruedas es la geometría Ackerman. La principal característica de esta geometría consiste en que evita el deslizamiento de las ruedas delanteras al aplicar un ángulo distinto a la rueda interior respecto a la exterior [30].

ICR Centro de rotación

$\mathrm{R}$ Radio de giro

1 Longitud entre ejes ruedas

b semi anchura vehículo

$\alpha$ ángulo rueda central

$\alpha_{0}$ ángulo rueda exterior

$\alpha_{\mathrm{i}}$ ángulo rueda interior

$\omega$ velocidad angular

$\theta$ ángulo entre sistemas coordenadas

$\mathrm{v}$ velocidad lineal

$\mathrm{v}_{\mathrm{t}}$ velocidad lineal transversal

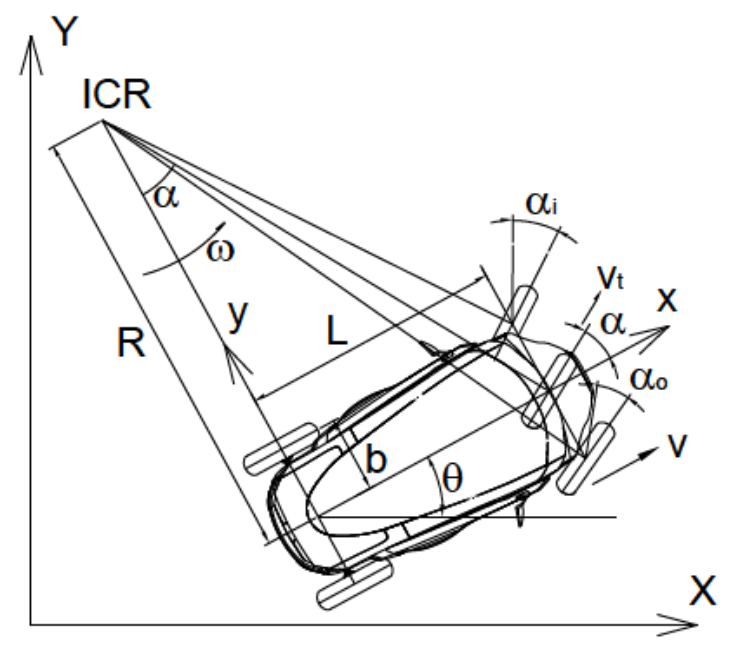

Figura 18. Modelo cinemático

En la Figura 18, se muestra el modelo cinemático para resolver las formulas a implementar.

Se determina la posición futura, en un tiempo $\mathrm{t}_{2}(\dot{x}, \dot{y}, \dot{\theta})$ del vehículo a partir de la velocidad lineal $(v)$ y angular $(\omega)$ que tiene en un tiempo actual $\mathrm{t}_{1}[31]$.

$$
\begin{aligned}
& \dot{x}=v \cdot \cos \theta \\
& \dot{y}=v \cdot \sin \theta \\
& \dot{\theta}=\omega,
\end{aligned}
$$


y de forma matricial

$$
\left[\begin{array}{c}
\dot{x} \\
\dot{y} \\
\dot{\theta}
\end{array}\right]=\left[\begin{array}{cc}
\cos \theta & 0 \\
\sin \theta & 0 \\
0 & 1
\end{array}\right]\left[\begin{array}{l}
v \\
\omega
\end{array}\right]
$$

la orientación actual del vehículo es $\theta$, la velocidad lineal y angular son, respectivamente

$$
v=v_{t} \cdot \cos \alpha_{\text {y }} \quad \omega=v \cdot \gamma
$$

donde $\gamma=\frac{1}{R}$ es la curvatura que describe el vehículo.

y de la Figura 18, de desprende

$$
\tan \alpha=\frac{l}{R}_{\mathrm{y}} \quad \gamma=\frac{\tan \alpha}{l}
$$

Desarrollando y expresando de forma matricial las ecuaciones de velocidad se tiene:

$$
\left[\begin{array}{c}
v \\
\omega
\end{array}\right]=\left[\begin{array}{c}
1 \\
\frac{\tan \alpha}{l}
\end{array}\right] v_{t} \cdot \cos \alpha
$$

Sustituyendo estos valores de $v$ y $\omega$ en la ecuación (2) se obtiene el modelo cinemático directo:

$$
\left[\begin{array}{c}
\dot{x} \\
\dot{y} \\
\dot{\theta}
\end{array}\right]=\left[\begin{array}{c}
\cos \theta \\
\sin \theta \\
\frac{\tan \alpha}{l}
\end{array}\right] v_{t} \cdot \cos \alpha
$$

Lo que interesa es obtener el modelo cinemático inverso. Aplicando la matriz pseudoinversa a la ecuación (5) se obtiene:

$$
\left[\begin{array}{l}
v_{t} \\
\alpha
\end{array}\right]=\left[\begin{array}{l}
\sqrt{v^{2}+\omega^{2} l^{2}} \\
\arctan \left(\frac{l \omega}{v}\right)
\end{array}\right]
$$

y sustituyendo en ésta los valores de la ecuación (2) obtenemos el modelo cinemático inverso

$$
\left[\begin{array}{c}
v_{t} \\
\alpha
\end{array}\right]=\left[\begin{array}{c}
\sqrt{(\dot{x} \cos \theta+\dot{y} \sin \theta)^{2}+\dot{\theta}^{2} l^{2}} \\
\arctan \left(\frac{\dot{\theta}}{\dot{x} \cos \theta+\dot{y} \sin \theta}\right)
\end{array}\right]
$$


Desde el punto de vista cinemático, la configuración Ackerman es equivalente a la del triciclo una vez se conoce la rueda equivalente a las dos ruedas delanteras.

La relación entre los ángulos de las ruedas interior y exterior es:

$$
\cot \alpha_{o}-\cot \alpha_{i}=\frac{R+b}{l}-\frac{R-b}{l}=\frac{2 b}{l}
$$

El ángulo de giro de la rueda equivalente puede calcularse mediante:

$$
\cot \alpha_{\mathrm{sa}}=\cot \alpha_{i}+\frac{b}{l}{ }_{\text {o bien }} \cot \alpha_{\mathrm{sa}}=\cot \alpha_{o}-\frac{b}{l}
$$

\subsection{PLANIFICACION DEL MOVIMIENTO Y SISTEMA DE CONTROL}

El objetivo en esta sección es alcanzar el destino deseado, de forma autónoma y segura. Con la ayuda de un mapa y un planificador global, calcula la mejor trayectoria. Esta trayectoria está marcada por un número reducido de puntos de referencia, que se definen por sus coordenadas GPS y la orientación del vehículo en el mapa. Para ejecutar maniobras de movimiento, un planificador local, calcula las trayectorias suaves, agregando puntos intermedios locales definidos por posición GPS, orientación, velocidades lineales y angulares. Un algoritmo de control define las acciones requeridas para llegar al siguiente punto de referencia local a lo largo de la trayectoria suave a la velocidad y orientación requeridas.

Debido a imprecisiones en el mapa o en la localización del vehículo, la trayectoria calculada podría cruzarse áreas no posibles. Además, objetos o el tráfico de la carretera pueden obstruir la trayectoria deseada. Los posibles peatones pueden cruzar la trayectoria del vehículo produciendo un riesgo potencial de colisión. Un módulo de detección de peatones puede calcular y proponer cambios de trayectoria.

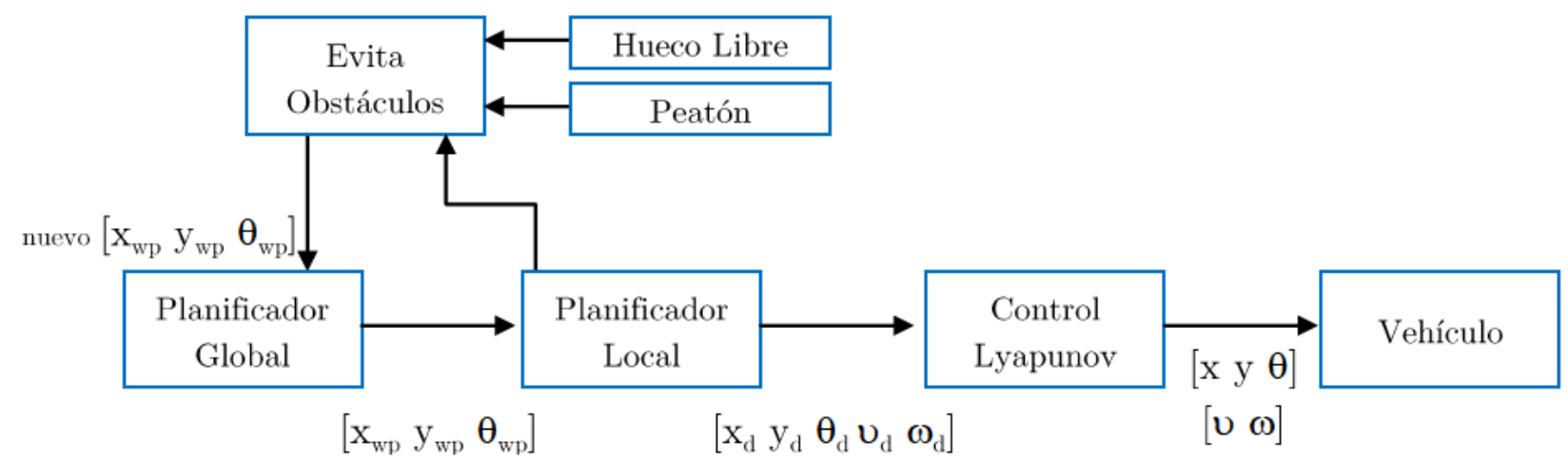

Figura 19. Esquema general de Sistema Control

El objetivo del sistema de control es traducir las acciones generadas por el sistema de toma de decisiones en acciones de los elementos que gobiernan el vehículo. El sistema de control se ha implementado con un compactRIO 9082 (de National Instruments). La unidad de procesamiento compactRIO está compuesta por dos procesadores: un INTEL i7 y un Xilinx FPGA, que confiere 
capacidades de alto rendimiento y flexibilidad para la prueba e implementación de algoritmos en el sistema de control. El compactRIO permite que se ejecuten procesos en tiempo real suaves y duros. El procesador INTEL permite que se ejecuten procesos menos críticos, mientras que FPGA permite que se ejecuten procesos críticos con frecuencias de reloj más altas. El sistema de control se rige por un sistema operativo en tiempo real (VxWorks de WindRiver). El núcleo del CICar está diseñado con máquinas de estado finito implementadas en $\mathrm{C}++$ a través del marco QS [32].

Los sistemas modificados son el volante Figura 20 (a), el pedal de freno Figura 20 (b) el pedal del acelerador y los botones D R N del sentido de la marcha. Para el volante, se ha instalado un doble engranaje en el eje de dirección, de modo que se puede girar usando un moto-reductor eléctrico. El pedal de freno se acciona mecánicamente mediante una leva controlada por un motoreductor eléctrico, con un ángulo de rotación máximo de $15^{\circ}$. El pedal del acelerador y los botones D R N son simulados electrónicamente por el sistema de procesado, que emula las señales analógicas electrónicas generadas por el pedal real. Ambos motores (volante y pedal de freno) son controlados por dos accionamientos a través de un bus CAN.

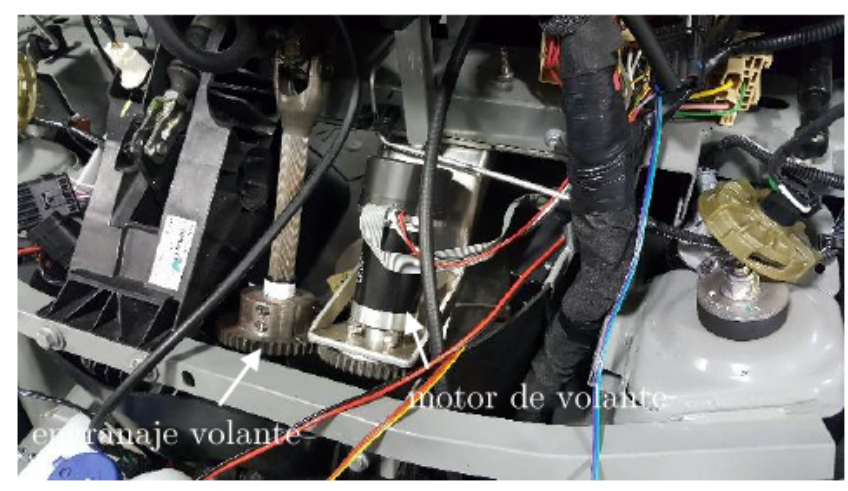

(a) volante

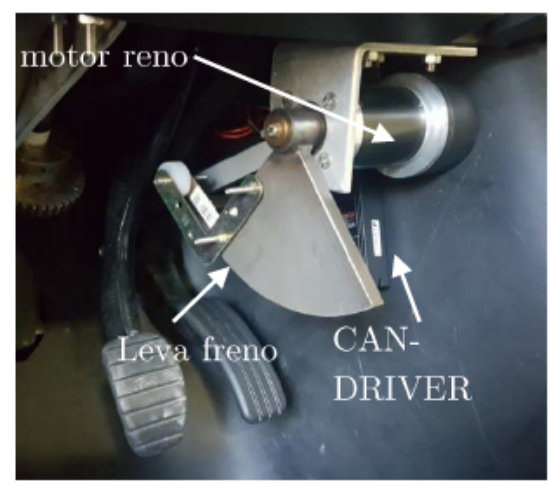

(b) pedal de freno

Figura 20. Sistema de control

\subsubsection{ALGORITMO DE CONTROL}

El algoritmo de control calcula las acciones de control necesarias para llegar al siguiente punto de referencia local a la velocidad y orientación requeridas. En [33] se propone un control de dirección PID anidado para mantener el carril en vehículos autónomos basados en visión para realizar el seguimiento de la ruta en el caso de carreteras con una curvatura incierta.

En [34] se presenta un diseño y validación experimental de un controlador lateral de vehículo autónomo basado en un control de modo deslizante de orden superior. Un interesante enfoque no lineal basado sobre los métodos de Lyapunov también se presenta en [35] para robots móviles terrestres.

Dado que se opera a una velocidad relativamente baja (máx. $20 \mathrm{~km} / \mathrm{h}$ ), se lograr un buen rendimiento utilizando un controlador basado en un modelo cinemático. Por lo tanto, se propone un control automático no lineal basado en el método de Lyapunov [36] para el seguimiento de la trayectoria entre puntos de referencia locales. Dicha estrategia de control se ha desarrollado y probado en el campo de la robótica, pero esta es la primera vez que se utiliza en el ámbito de los vehículos autónomos. El objetivo de control del seguimiento de trayectoria es alcanzar asintóticamente una diferencia nula entre la posición y la orientación del automóvil real con respecto a la ruta. La idea básica del método Lyapunov es definir una ley de control que asegure la estabilidad y la eliminación asintótica del error cometido por el automóvil real cuando se siguen los puntos de referencia previamente especificados. 
Esta técnica de control utiliza un modelo cinemático de bicicleta definido por las siguientes ecuaciones para el automóvil real y virtual

$$
\left\{\begin{array}{l}
\dot{x}=v \sin (\theta) \\
\dot{y}=v \cos (\theta) \\
\dot{\theta}=\frac{v}{l_{f}} \tan (\delta)
\end{array}\right.
$$

donde $\mathrm{x}, \mathrm{y}$ y $\boldsymbol{\theta}$ representan la posición y orientación actuales del vehículo en metros y radianes respectivamente, $v$ es la velocidad lineal, $\delta$ representa el ángulo de dirección y $l_{\mathrm{f}}$ se refiere a la distancia entre el centro de masa del vehículo y las ruedas frontales eje.

El vector de error se define como la diferencia entre las mediciones reales y los valores deseados multiplicados por la matriz de rotación sobre el eje Z, que es ortogonal al plano de la carretera:

$$
\left[\begin{array}{l}
x_{e} \\
y_{e} \\
\theta_{e}
\end{array}\right]=\left[\begin{array}{ccc}
\cos \left(\theta_{d}\right) & \sin \left(\theta_{d}\right) & 0 \\
-\sin \left(\theta_{d}\right) & \cos \left(\theta_{d}\right) & 0 \\
0 & 0 & 1
\end{array}\right] *\left[\begin{array}{c}
x-x_{d} \\
y-y_{d} \\
\theta-\theta_{d}
\end{array}\right]
$$

La derivación del vector de error proporciona el siguiente sistema de error de bucle abierto:

$$
\begin{aligned}
& \dot{x}_{e}=v \cos \left(\theta_{e}\right)+y_{e} \frac{v_{d}}{l_{f}} \tan \left(\delta_{d}\right)-v_{d} \\
& \dot{y}_{e}=v \sin \left(\theta_{e}\right)-x_{e} \frac{v_{d}}{l_{f}} \tan \left(\delta_{d}\right) \\
& \dot{\theta}_{e}=\frac{v}{l_{f}} \tan (\delta)-\frac{v_{d}}{l_{f}} \tan \left(\delta_{d}\right)
\end{aligned}
$$

Ahora se puede definir cómo funciona el controlador para mantener una velocidad constante y maniobrar cómodamente. Recibe los puntos de ajuste de la trayectoria proporcionada por el planificador local $\left(\boldsymbol{v}_{\mathrm{d}} \mathrm{y} \omega_{\mathrm{d}}\right)$, los tres errores definidos en la ecuación 21, y la velocidad $(\mathbf{v})$ y la velocidad angular del centro de gravedad del vehículo $(\omega)$ como acciones de control.

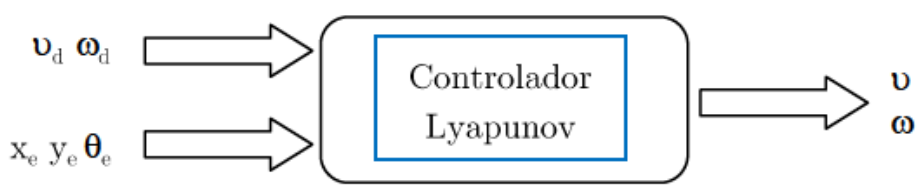

Figura 21. Diagrama de controlador basado en Lyapunov 
El modelo de error se calcula a partir de (21) derivando las leyes de control. Después de evaluar la derivada de tiempo del modelo de error (22) y aplicar algunas operaciones, se logra el siguiente modelo de error dinámico:

$$
\left[\begin{array}{c}
\dot{e}_{1} \\
\dot{e}_{2} \\
\dot{e}_{3}
\end{array}\right]=\left[\begin{array}{c}
\dot{\theta} e_{2}+\mu_{1} \\
-\dot{\theta} e_{1}+v_{d} \sin \left(e_{3}\right) \\
\mu_{2}
\end{array}\right]
$$

donde $\mu=\left[\mu_{1} \mu_{2}\right]^{\mathrm{T}} \in \mathrm{R}^{2}$ es un vector de entrada auxiliar definido como:

$$
\left[\begin{array}{l}
\mu_{1} \\
\mu_{2}
\end{array}\right]=\left[\begin{array}{c}
v_{d} \cos \left(e_{3}\right) \\
\dot{\theta}_{d}
\end{array}\right]-\left[\begin{array}{l}
v \\
\dot{\theta}
\end{array}\right]
$$

donde el primer término es un vector auxiliar y el segundo término corresponde a las acciones de control deseadas. Basado en (23) y el posterior desarrollo del sistema de error de circuito cerrado, se puede extraer el vector de entrada auxiliar, obteniendo la siguiente expresión:

$$
\left[\begin{array}{l}
\mu_{1} \\
\mu_{2}
\end{array}\right]=\left[\begin{array}{c}
-k_{1} x_{e} \\
-k_{2} v_{d} \frac{\sin \left(\theta_{e}\right)}{\theta_{e}} y_{e}-k_{3} \theta_{e}
\end{array}\right]
$$

donde $\mathrm{k}_{1}, \mathrm{k}_{2} \mathrm{y} \mathrm{k}_{3} \in \mathrm{R}^{1}$ son constantes $\mathrm{y}$ ganancias positivas para asegurar la estabilidad asintótica del circuito cerrado. Restando las variables de control de (24), se obtienen las siguientes leyes de control:

$$
\left[\begin{array}{c}
v \\
\omega
\end{array}\right]=\left[\begin{array}{c}
v_{d} \cos \left(\theta_{e}\right)+k_{1} x_{e} \\
\dot{\theta}_{d}+k_{2} v_{d} \frac{\sin \left(\theta_{e}\right)}{\theta_{e}} y_{e}+k_{3} \theta_{e}
\end{array}\right]
$$

Sustituyendo (25) en (26), se obtiene el siguiente sistema de error de circuito cerrado:

$$
\left[\begin{array}{c}
\dot{e}_{1} \\
\dot{e}_{2} \\
\dot{e}_{3}
\end{array}\right]=\left[\begin{array}{c}
\dot{\theta} y_{e}-k_{1} x_{e} \\
-\dot{\theta} x_{e}+v_{d} \sin \left(\theta_{e}\right) \\
-k_{2} v_{d} \frac{\sin \left(\theta_{e}\right)}{\theta_{e}} y_{e}-k_{3} \theta_{e}
\end{array}\right]
$$

El teorema de Lyapunov para la estabilidad local y global se utiliza para asegurar la estabilidad del sistema. Usando el siguiente candidato a la función Lyapunov:

$$
V(e)=\frac{k_{2}}{2} e_{1}^{2}+\frac{k_{2}}{2} e_{2}^{2}+\frac{1}{2} e_{3}^{2}
$$


Y la derivada de este candidato:

$$
\dot{V}(e)=k_{2} e_{1} \dot{e}_{1}+k_{2} e_{2} \dot{e}_{2}+e_{3} \dot{e}_{3}
$$

se asegura la estabilidad global siempre que $\mathrm{k}_{1}, \mathrm{k}_{2}$ y $\mathrm{k}_{3}$ sean constantes definitivas positivas. 
4 CAPITULO 4

\section{SISTEMAS DE PERCEPCION}

\subsection{INTRODUCCION}

Los rápidos avances en la tecnología de la electrónica, la información y las comunicaciones (que conducen a la miniaturización y mejora del rendimiento de los ordenadores, sensores y redes) han dado lugar al desarrollo de varias tecnologías de vehículos autónomos (VAs) [37]. Paralelamente a esta evolución, los procesos y procedimientos para probar funciones VAs también se han desarrollado y establecido durante las décadas anteriores, de acuerdo con los requisitos de las funciones y la normativa establecida. Además, para introducir vehículos autónomos en áreas urbanas, existen algunas preocupaciones como las posibles consecuencias, especialmente para la seguridad de las personas, ante un fallo mecánico que pueda causar un choque. Para garantizar que sea seguro para VAs y reducir costos, se deben modelar y probar diferentes escenarios. Las pruebas sistemáticas de vehículos autónomos se pueden realizar en simulación o en el mundo físico. Las pruebas físicas ofrecen la ventaja de que el mundo real está lleno de escenarios de prueba y los ingenieros pueden utilizar el vehículo real en lugar de crear modelos. Sin embargo, algunas regulaciones restringen el uso de VAs en las ciudades y además para realizar pruebas con VAs reales, se necesita acceso a hardware costoso y pruebas de campo que generalmente consumen una cantidad considerable de tiempo. Según informes publicados recientemente [38], se considera que sería imposible realizar pruebas de campo empíricas que verifiquen la seguridad de un coche autónomo en un plazo razonable. En este contexto, la simulación, el modelado y las pruebas tienen el potencial de llenar este vacío y permitir sistemas una evaluación rigurosa, controlada y oportuna de los sistemas de conducción autónoma.

Este capítulo se dedica al sistema de percepción de los vehículos autónomos de mayor autonomía y complejidad, enfatizando sus subsistemas [39]: Percepción del entorno y Localización (Figura 22).

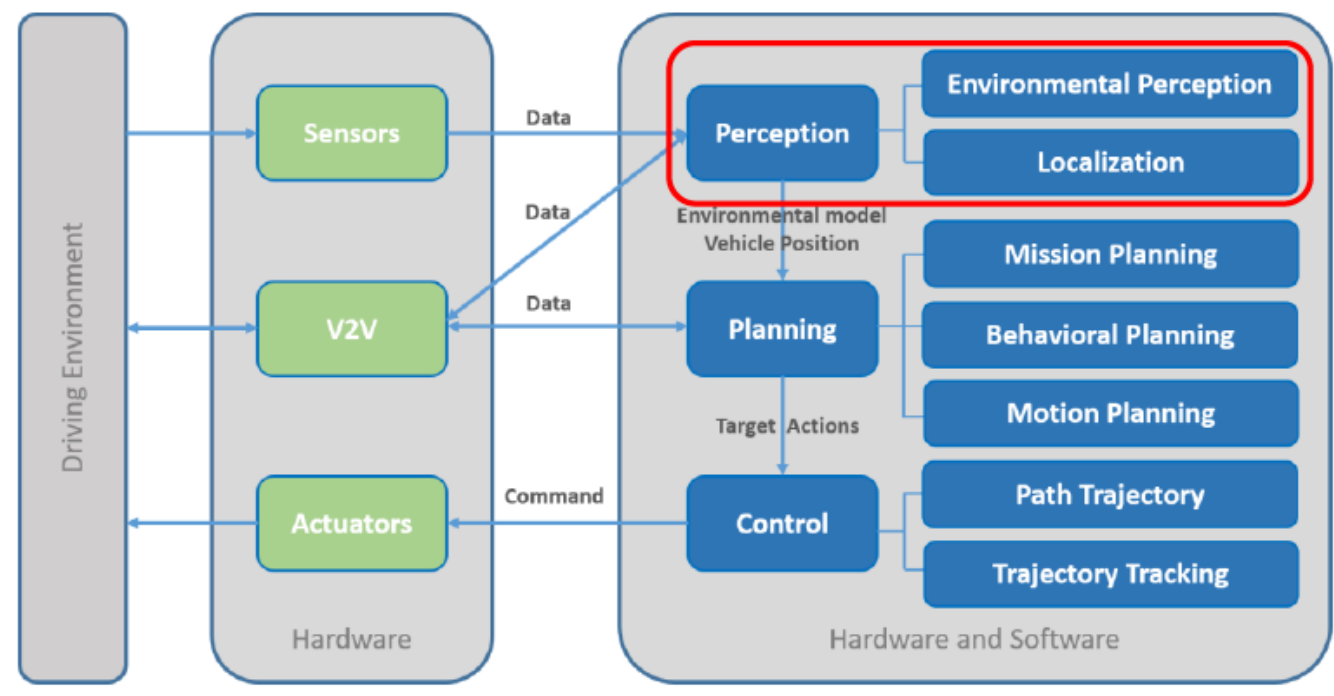

Figura 22. Sistema típico de vehículo autónomo 
Se presentan en detalle todos los aspectos tecnológicos y heredados que intervienen en el desarrollo de VAs y se especifican las diferentes alternativas disponibles para simular y probar cada uno de los subsistemas.

\subsection{SISTEMA DE PERCEPCIÓN DEL ENTORNO PARA}

\section{$\underline{\text { VAS }}$}

Un vehículo autónomo adquiere conocimiento del mundo circundante en dos etapas. La primera etapa consiste en escanear la carretera para detectar posibles cambios en las condiciones de conducción (semáforos y señales, paso de peatones, barreras...). La segunda etapa se relaciona con la percepción de los otros vehículos.

En este apartado se presentan los sensores más representativos que componen los sistemas de percepción de los VAs: ultrasónicos, RADAR, LIDAR 3D, cámaras, IMU, GNSS, RTK. Existen numerosos artículos o libros científicos que muestran los tipos de sensores utilizados en VAs, sus aplicaciones, así como sus ventajas y desventajas. Estos trabajos muestran los sensores como cajas negras formadas por un conjunto de entradas y salidas, sin ahondar en los fundamentos físicos de su funcionamiento. Como aspecto novedoso de este capítulo, los sensores se presentan desde el punto de vista del espectro electromagnético que utilizan activa o pasivamente para su funcionamiento. Esto permitirá adquirir un conocimiento más profundo de las virtudes y desventajas de los mismos que se adquieren al trabajar en ambientes degradados o condiciones climáticas adversas. La Figura 23 muestra el espectro electromagnético dividido en dos escalas, longitudes de onda y frecuencias. Además, se muestran los rangos espectrales de los sensores utilizados en los VAs.

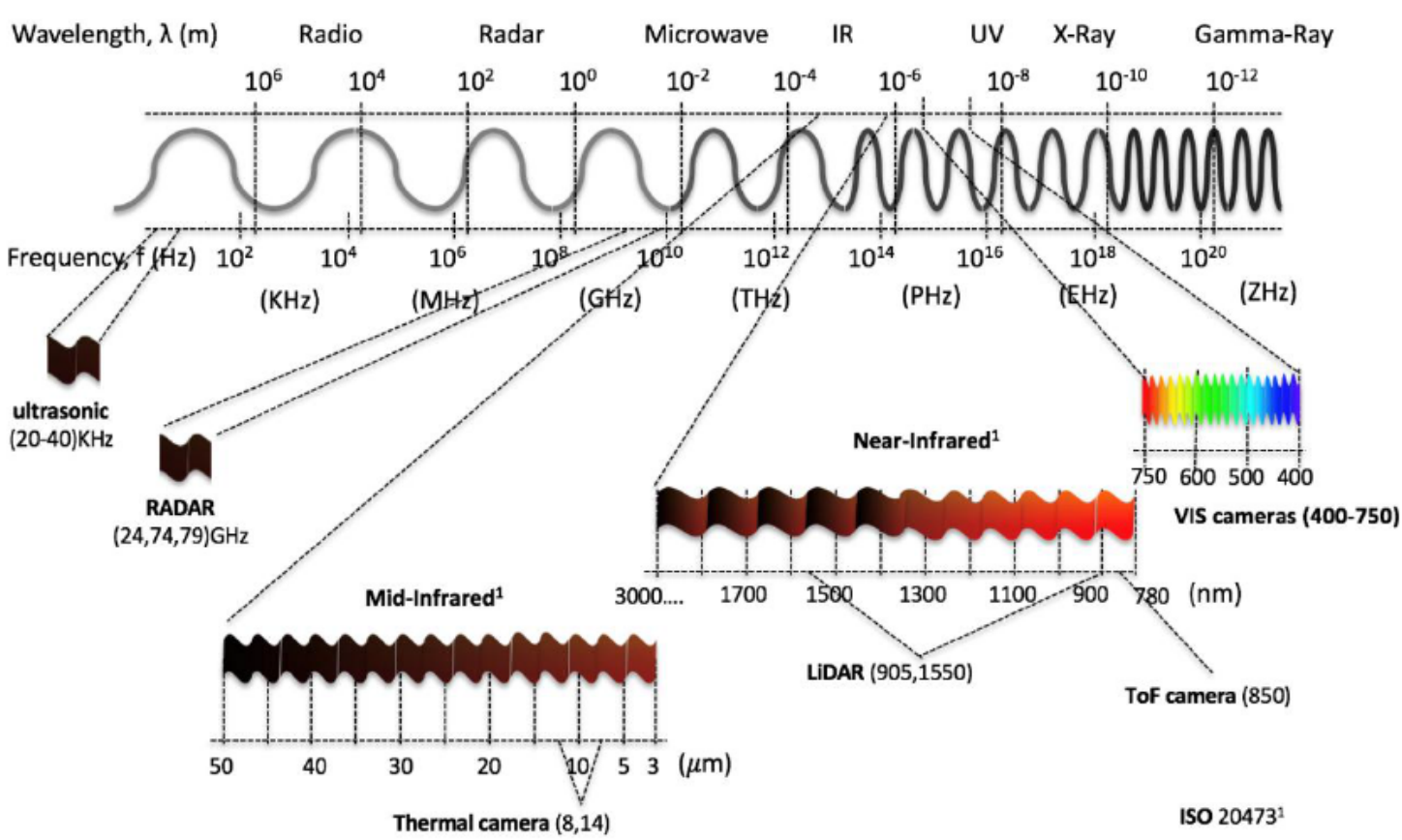

Figura 23. Descripción general diferentes espectros utilizados para el sistema de percepción de vehículos autónomos 


\subsubsection{ULTRASONICOS}

Como su nombre lo indica, los sensores ultrasónicos utilizan ondas sónicas, en el rango entre $20 \mathrm{kHz}$ a $40 \mathrm{kHz}$, generadas por una membrana magnetorresistiva para medir la distancia a un objeto. Su principio de funcionamiento se basa en la medición del tiempo de vuelo (ToF) de la onda sónica desde que se emite hasta que se recibe el eco.

$$
d=\frac{c}{2} * \mathrm{ToF}
$$

Siendo la velocidad c de la onda en metros por segundo y ToF es el tiempo de vuelo en segundos.

Estos sensores se suelen utilizar en entornos industriales para la medida de alturas en almacenamiento de todo tipo de materias primas. En vehículos se utilizan en sistemas de estacionamiento o como sensores de medición de distancias cortas a bajas velocidades (Figura 24a y 24b). Estos sensores tienen un bajo costo y producen buenos resultados en la medición de distancias con cualquier material independientemente de su color, en ambientes polvorientos o en condiciones climáticas adversas (humedad o lluvia). Poseen la desventajas que tienden a producir falsos positivos por rebote, además poseen una zona ciega en la medición, denominada blanking, ubicada entre el elemento emisor del sensor y el rango mínimo.
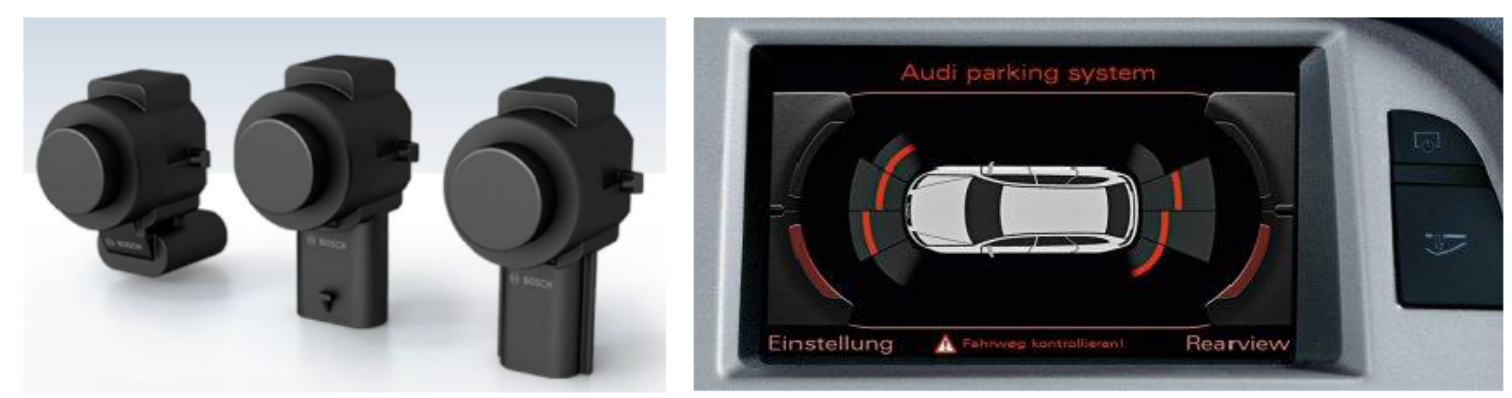

Figura 24. (a) Sensores ultrasónicos para automóviles de Bosh; (b) Sistema de asistencia al estacionamiento de Audi

\subsubsection{RADAR (RADIO DETECTION AND RANGING)}

Los sistemas de Radio Detection And Ranging (RADAR) funcionan en longitudes de onda del orden de milímetros, estos se utilizan en una amplia variedad de aplicaciones militares y civiles como sistemas de detección de amenazas aéreas o terrestres, sistemas de tiro, aeropuertos o sistemas meteorológicos. La aparición de vehículos inteligentes y la necesidad de incrementar la seguridad vial han impulsado el uso de este tipo de dispositivos en el sector de la automoción. Los sistemas de radar para vehículos inteligentes funcionan a frecuencias de $24 / 77 / 79 \mathrm{GHz}$ y se conocen como radar de ondas milimétricas (MMW). El radar mide la distancia entre el emisor y el objeto calculando el tiempo de vuelo de la señal emitida y el eco recibido. Los radares no solo permiten la detección de la distancia a varios objetivos, sino que también son capaces de proporcionar con precisión la dirección y velocidad de los objetivos. Los nuevos radares para vehículos utilizan una matriz de micro antenas capaces de generar un conjunto de lóbulos que permiten la mejora del alcance y un sistema de procesamiento para la detección de múltiples objetivos (Figura 25a y 25b). 


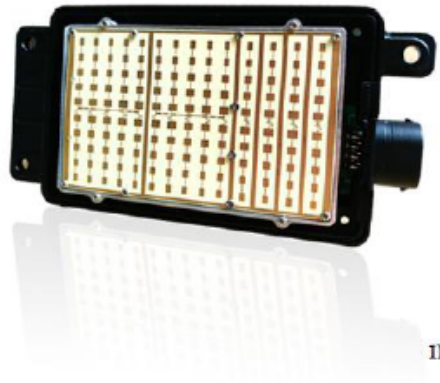

(a)

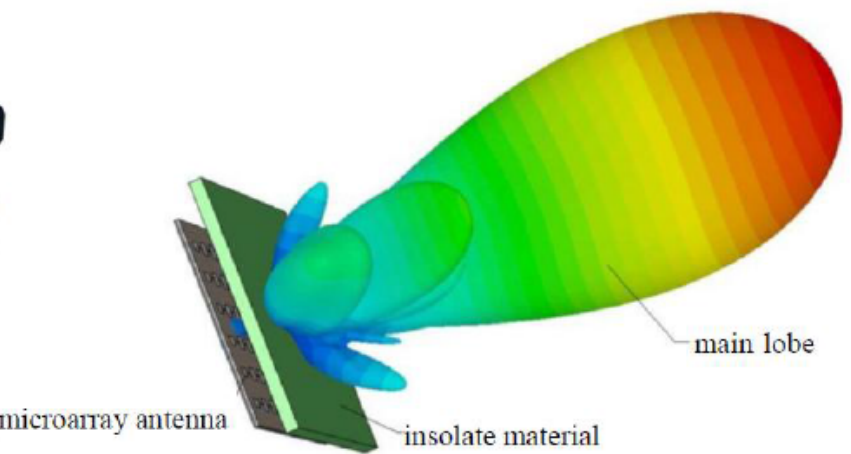

(b)

Figura 25. (a) RADAR CAR70 de ondas milimétricas de Nanoradar: (a) Antena de radar de microarrays; (b) Sistema multilobular

El RADAR de onda milimétrica de rango se aplica en detección de punto ciego (BSD), asistente de cambio de carril (LCA), alerta de tráfico cruzado trasero (RCTA), alerta de tráfico cruzado delantero (FCTA) o fusión de video por radar. Las ondas de radar tienen una mayor penetrabilidad porque tienen buenas características en todo clima, pueden detectar con precisión los objetivos de corto alcance en la parte delantera, lateral y trasera del vehículo. Por esta razón, se utilizan en muchos de los sistemas ADAS. El RADAR puede mejorar significativamente la seguridad del vehículo, reducir la carga de toma de decisiones de las personas al volante y puede ser instalado oculto tras los parachoques del vehículo. Algunas desventajas del tipo de sensor son la falta de precisión, su campo de visión reducido (FOV) y puede producir falsos positivos debido a los rebotes de la señal emitida.

\subsubsection{LIDAR (LASER IMAGING DETECTION AND RANGING)}

Los sistemas LIDAR fueron desarrollados inicialmente en los años 70 para la medición de elementos en mar o tierra desde satélites o aviones, fue desarrollado específicamente para la detección de submarinos por la Armada Estadounidense. Los sistemas LIDAR basan su funcionamiento en la medición del tiempo de vuelo de una luz pulsada emitida por un diodo láser hasta que es recibida por un emisor. Los rangos de emisión están en el infrarrojo (905 nm o 1550 $\mathrm{nm})$. Las emisiones a $905 \mathrm{~nm}$ requieren menos energía que las emitidas a $1550 \mathrm{~nm}$ porque el agua en la atmósfera comienza a absorber energía a partir de $1400 \mathrm{~nm}$. Esta desventaja inicial del aumento de potencia a $1550 \mathrm{~nm}$ es aprovechada por el líquido acuoso del ojo para filtrar totalmente esta longitud de onda, haciéndolos menos dañinos que los LIDAR a $905 \mathrm{~nm}$. Los láseres utilizados para vehículos pertenecen a la clase 1 y son seguros en todas las condiciones de uso normal. Los LIDAR utilizan el principio ToF para realizar la medición de la distancia entre la emisión y la recepción. Estos se pueden clasificar según el tipo de información que obtienen de su entorno en LIDARs 2D o 3D o se pueden clasificar según su construcción en LIDAR rotativos o de estado sólido. El LIDAR 2D obtiene información del entorno proyectando un solo haz láser en un espejo giratorio perpendicular al eje de rotación (ver Figura 26a). El LIDAR 3D permite obtener un mapa 3D de gran precisión del entorno, para ello utilizan un conjunto de diodos láseres montados en un vástago que gira a gran velocidad (ver Figura 26b). El número de láseres instalados en el vástago determina la precisión de la nube de puntos obtenida en cada vuelta. Actualmente podemos encontrar LIDAR 3D que integran de 4 a 128 láseres o canales con un FOV horizontal de 360 grados y un FOV vertical que oscila entre 20-45 grados con precisión de unos pocos centímetros. Dependiendo del número de canales, el LIDAR 3D se utiliza en el control de crucero adaptativo (ACC), la evitación de objetos, la identificación de objetos o el mapeo 3D. El LIDAR se ve afectado por condiciones climáticas como la lluvia, la nieve, la niebla o ambientes polvorientos debido a la 
difracción de la luz en ellos. Además, reducen su rango de detección en función de la reflectividad de los objetos a los que inciden los haces láser. La capacidad máxima de detección en función del tipo de reflectividad del material a detectar se presenta en la hoja de datos proporcionada por el fabricante, por ejemplo, LIDAR Velodyne HDL64E ofrece un rango de detección de $50 \mathrm{~m}$ para objetos con una reflectividad 0,1 (pavimento) y un rango hasta $120 \mathrm{~m}$ para objetos con un reflectividad 0,8 (vehículos y vegetación).

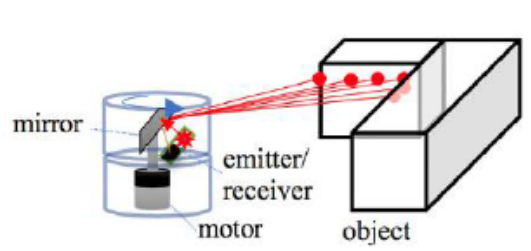

(a)

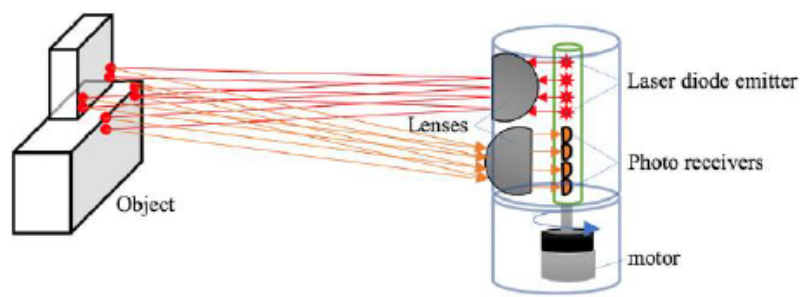

(b)

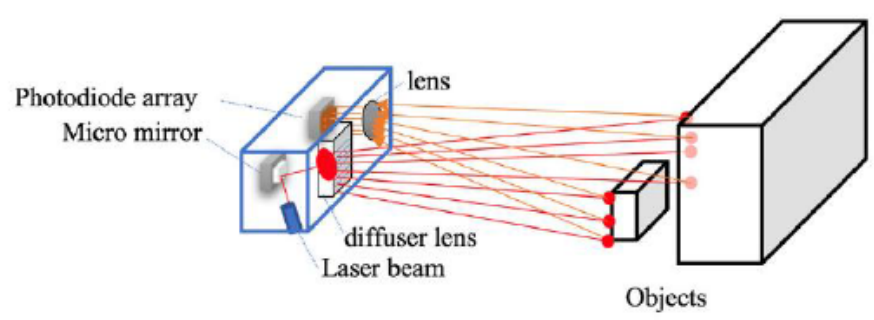

(c)

Figura 26. Esquemas operativos (a) LIDAR 2D giratorio, (b) LIDAR 3D giratorio, (c) LIDAR 3D de estado sólido

El último tipo de dispositivo basado en tecnología láser que ha llegado al campo desarrollo de los vehículos autónomos, es el LIDAR de estado sólido. El LIDAR de estado sólido permite obtener una representación en 3D de la escena sin el uso de partes móviles en el dispositivo. Un circuito Micro-electro-mechanical Systems (MEMS) de microespejo realiza la sincronización con el haz láser para escanear el FOV horizontal en múltiples líneas. Para eso, el micro-espejo refleja el haz sobre una lente difusora que crea una línea vertical que impactará contra los objetos (Figura 26c). La luz reflejada es capturada por una lente y se envía a una matriz de fotodetectores para construir la primera línea de una matriz 3D. El proceso se repite hasta que se crea una nube de puntos de la escena. Esta característica aumenta notablemente su durabilidad, reduce las tareas de mantenimiento y su precio. El LIDAR de estado sólido tiene un campo de visión más pequeño que el LIDAR giratorio pero como la tendencia del mercado es sustituir el LIDAR 3D giratorio por un conjunto de LIDAR de estado sólido integrados alrededor del vehículo.

\subsubsection{CAMARAS}

En el sistema de percepción en vehículos autónomos y desde el punto de vista de la longitud de onda que recibe el dispositivo, las cámaras se pueden clasificar en visibles (VIS) o infrarrojas (IR). El elemento utilizado por la cámara para capturar la escena se conoce como sensor de imagen y tradicionalmente se ha implementado con dos tecnologías: dispositivo de carga acoplada (CCD) y semiconductor de óxido metálico complementario (CMOS). Los sensores de imagen CCD se fabrican mediante un costoso proceso de fabricación que les confiere propiedades únicas, como alta eficiencia de cuantificación y bajo nivel de ruido. CMOS fue desarrollado para reducir el costo de fabricación a expensas de reducir su rendimiento. Respecto a la tecnología CCD, el diseño de la arquitectura CMOS de extracción de los valores de luminosidad permite la selección y procesamiento de regiones de interés (ROI), además el dispositivo CMOS tiene un consumo 
menor que los CCD. Estas características los convierten en los más utilizados para dispositivos móviles. Por otro lado, la tecnología CCD tiene un alto rango dinámico y una mayor calidad de imagen en entornos con poca luz. Las diferencias de ambas tecnologías comienzan a superponerse y se espera que en el futuro la tecnología CMOS sustituya al CCD.

Las cámaras VIS capturan longitudes de onda entre $400 \mathrm{~nm}$ y $780 \mathrm{~nm}$ de la misma forma que el ojo humano. El espectro visible se divide en tres bandas o canales: R, G y $B$ que se codificarán por separado. Estos dispositivos son los más utilizados en los sistemas de percepción de los VAs para obtener información sobre el entorno del vehículo debido a su bajo costo, alta calidad de información en color y alta resolución. El enorme volumen de datos generados por medio del dispositivo supone un problema adicional para el sistema de procesamiento. Las aplicaciones más comunes son detección de punto ciego (BSD), asistente de cambio de carril (LCA), control de vista lateral, registro de accidentes, identificación de objetos y detección de señales. Las cámaras VIS se ven muy afectadas por las variaciones en las condiciones de iluminación, lluvia, nieve o niebla, por lo que se combinan con las tecnologías RADAR y LIDAR para aumentar su robustez.

La combinación de dos cámaras VIS con una distancia focal conocida, permite realizar la visión estereoscópica, lo que agrega un nuevo canal llamado información de profundidad. Las cámaras con estas características se conocen como RGBD y proporcionan una representación en 3D de la escena alrededor del vehículo.

Las cámaras IR son sensores pasivos que funcionan en rangos de longitudes de onda infrarrojas (IR) entre $780 \mathrm{~nm}$ y $1 \mathrm{~mm}$. Hay muchos dispositivos que funcionan en este espectro porque existen menos interferencias de luz (por ejemplo, LIDAR). Los sistemas de percepción que incluyen cámaras IR funcionan en infrarrojo cercano (NIR: $780 \mathrm{~nm}-3 \mathrm{~mm}$ ) o en infrarrojo medio (MIR: $3 \mathrm{~mm}-50 \mathrm{~mm}$, conocidas como cámaras térmicas). El uso de NIR suele sustituir o complementar a las cámaras VIS. Las cámaras de infrarrojos se utilizan: (1) en situaciones en las que hay picos de iluminación, por ejemplo: en la salida de un túnelo o cuando se conduce frente al sol; y (2) en la detección de cuerpos calientes, como peatones, animales u otros vehículos. En estos casos, las cámaras térmicas permiten simplificar el proceso de segmentación a operaciones basadas en umbrales, además éstas no se ven afectadas por las condiciones climáticas y de iluminación. Por otro lado, proporcionan una imagen en escala de grises y el gran tamaño de celda del sensor de imagen reduce notablemente su resolución. 
Las cámaras ToF son sensores activos que utilizan el principio de tiempo de vuelo para obtener una representación 3D de los objetos en la escena. Las cámaras ToF emiten pulsos de luz NIR de $850 \mathrm{~nm}$ con una matriz de LED (diodos emisores de luz) y miden la diferencia de fase $\Delta \varphi$ entre la señal modulada emitida (sE) y la señal recibida (sR) para calcular la distancia como se muestra en la ecuación 30 y Figura 27.

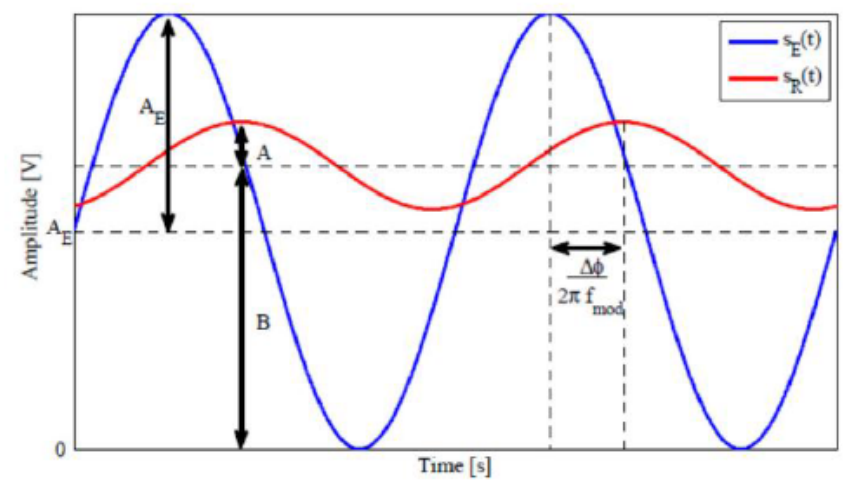

Figura 27. Señal emitida (azul) y señal recibida (rojo).

$$
d=\frac{c}{2} * \frac{\Delta \varphi}{2 \pi f_{\text {mod }}}
$$

En las cámaras ToF la distancia varía entre 10 metros para escenas de interior y unos 4 metros para escenas de exterior, dependiendo de la cantidad de LED en la matriz. Al igual que las cámaras IR, tienen una resolución baja debido a las características de la longitud de onda que deben capturar.

\subsubsection{COMPARATIVA SENSORES SISTEMA PERCEPCION}

En la Tabla 2 se muestra un resumen de las ventajas y desventajas de los sensores analizados en este apartado. En la tabla se muestran once características, las cuales han sido cuantificadas con los datos obtenidos en esta revisión. La cuantificación de las características de los sensores se ha realizado mediante cuatro valores para simplificar el proceso: 0- ninguna, 1- baja, 2media y 3- alta.

\begin{tabular}{lccccccc}
\hline & Ultrasonic & RADAR & \multicolumn{2}{c}{ LIDAR } & \multicolumn{3}{c}{ Cámeras } \\
\hline & & & Rotating & Solid State & VIS & IR & ToF \\
FOV & 1 & 2 & 3 & 2 & 3 & 3 & 2 \\
Range & 1 & 3 & 3 & 3 & 2 & 3 & 2 \\
Accuracy & 1 & 2 & 3 & 3 & 3 & 2 & 2 \\
Frame rate & 2 & 2 & 2 & 2 & 2 & 3 & 3 \\
Resolution & 1 & 1 & 2 & 2 & 3 & 1 & 1 \\
Colour perception & 0 & 0 & 1 & 2 & 3 & 1 & 1 \\
Size & 1 & 1 & 2 & 1 & 1 & 1 & 1 \\
Weather affections & 1 & 1 & 2 & 2 & 3 & 1 & 3 \\
Maintenance & 2 & 1 & 2 & 1 & 2 & 2 & 2 \\
Visibility & 2 & 1 & 3 & 2 & 2 & 2 & 2 \\
Price & 1 & 2 & 3 & 1 & 1 & 3 & 2 \\
\hline
\end{tabular}

Tabla 2. Resumen de las principales características de los sensores utilizados en los sistemas de percepción de VAS 
La Figura 28 muestra un conjunto de gráficos de araña de las características de los sensores presentados en esta revisión con respecto al sensor perfecto. El sensor perfecto se ha definido como aquel que obtendría mejores puntuaciones en todas las características analizadas en esta revisión. Esto significa valores máximos (3) para FOV, rango, precisión, velocidad de fotogramas, resolución, percepción del color y valores mínimos (0) para efectos climáticos, mantenimiento, visibilidad y precio. Esta comparación permite conocer los aspectos positivos y los puntos débiles de un vistazo.
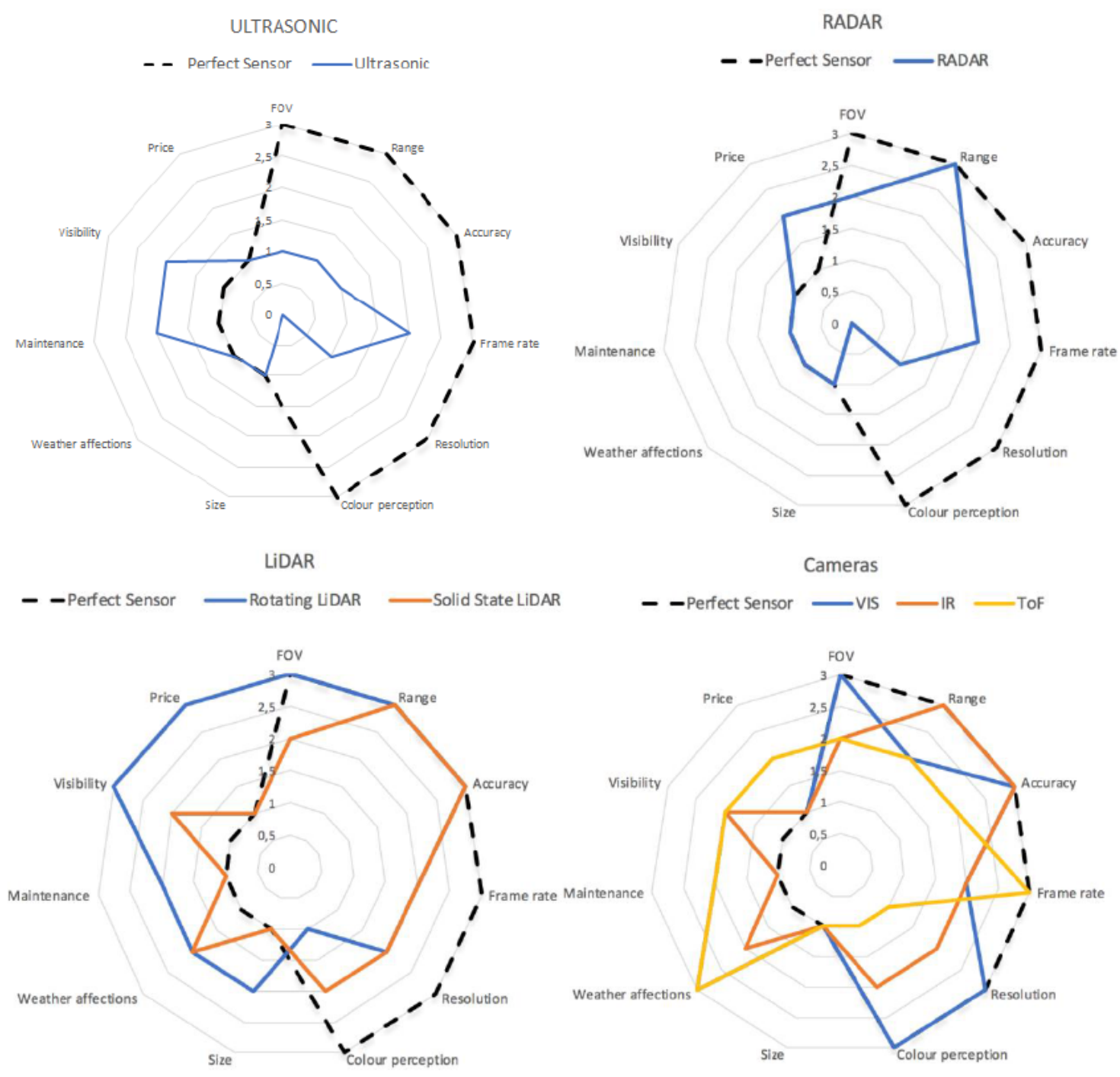

Figura 28. Gráficos araña de sistemas de percepción

\subsection{SISTEMAS DE POSICIONAMIENTO PARA VAS}

El sistema de navegación global por satélite (GNSS) es la tecnología de posicionamiento más utilizada para vehículos en tierra, mar y aire. El GNSS proporciona la posición absoluta de un receptor con respeto a una referencia fija y consiste en un conjunto de satélites que orbitan aproximadamente a $20.000 \mathrm{~km}$ de la superficie terrestre. Estos emiten señales con información sobre el satélite, su posición, parámetros orbitales, etc. Este sistema se completa con sistemas de recepción, que reciben esas señales y extraen información sobre la posición, la velocidad y el tiempo exacto. El sistema GNSS más conocido es el Sistema de Posicionamiento Global. o GPS, desarrollado por los EEUU en la década de 1970, que consta de 24 satélites ubicados en seis planos separados por $55^{\circ}$ y con un periodo de rotación de 11 horas y 58 minutos. Su configuración permite 
que cualquier receptor ubicado en la superficie de la tierra pueda recibir señales de entre 6 y 12 satélites. El principio de funcionamiento del GNSS se basa en la medición del tiempo de vuelo de la señal emitido por el satélite y el recibido por el receptor. El sistema es capaz de obtener de forma fiable los detalles de posición y tiempo $(\mathrm{x}, \mathrm{y}, \mathrm{z}, \mathrm{t})$ con un mínimo de cuatro satélites visibles. La Tabla 3 muestra las características de los sistemas GNSS más utilizados para el posicionamiento global, sus constelaciones, precisión, cobertura, período de rotación, altura y propietario [40].

\begin{tabular}{ccccc}
\hline & GPS & GLONASS & GALILEO & BEIDOU \\
\hline Satellites & 24 & 24 & 30 & $30+5$ \\
Precision & 7.8 civil & 7.4 civil & 1.0 civil & 10.0 civil \\
Period & $11 \mathrm{~h} 58 \mathrm{~m}$ & $11 \mathrm{~h} 15 \mathrm{~m}$ & $14 \mathrm{~h} 00 \mathrm{~m}$ & $12 \mathrm{~h} 53 \mathrm{~m}$ \\
Heigh & $26.650 \mathrm{~km}$ & $19.100 \mathrm{~km}$ & $23.222 \mathrm{~km}$ & $21.150 \mathrm{~km}$ \\
Owner & EEUU & Rusia & EU & China \\
\hline
\end{tabular}

Tabla 3. Algunas plataformas para el desarrollo de conducción autónoma

Las señales de los satélites están influenciadas por numerosos errores:

(1) sincronización de los relojes atómicos de los satélites en órbita

(2) la transmisión de señales por la ionosfera y la troposfera

(3) el ruido en los receptores

(4) efecto de trayectoria múltiple debido a reflexiones de señal

(5) incertidumbres geométricas.

El GNSS diferencial (DGNSS) se desarrolló para aliviar los errores que afectan la medición de la señal de constelaciones de satélites. El DGNSS consta de dos receptores GNSS, una estación base o tierra y una estación móvil, conocida como Rover. La estación base conoce su posición exacta y continuamente comunica las correcciones de señal a la estación móvil. Las correcciones de señal proporcionan precisiones. de $0,7 \mathrm{~m}$ a $3 \mathrm{~m}$ en aplicaciones civiles y mejorar la integridad de la medición (capacidad del sistema para proporcionar advertencias oportunas a los usuarios cuando el sistema no debe utilizarse para la navegación). DGNSS necesita un sistema de estaciones terrestres georeferenciadas con precisión y un sistema de comunicación, usualmente radio UHF, con las estaciones móviles.

Existen otros sistemas basados en la corrección de la señal que provienen de los satélites, como el "Satellite Based Augmentation System" (SBAS) y la cinemática en tiempo real (RTK).

El sistema SBAS fue diseñado para mejorar la navegación aérea al aumentar la precisión horizontal y vertical de los receptores y proporcionando información sobre la calidad de las señales. Para este propósito, tiene un conjunto de estaciones distribuidas en grandes áreas geográficas que monitorean el estado de las constelaciones de satélites, informando de cualquier anomalía. Estos sistemas son operados por diferentes instituciones, gobiernos e incluso empresas privadas [41].

Los sistemas RTK utilizan la portadora de señal satelital para mejorar la precisión de posición del Rover. Estos sistemas obtienen precisiones de hasta $2 \mathrm{~cm}$ y usualmente usan un radio módem para comunicarse entre estaciones base y Rovers dentro de un rango de $20 \mathrm{Km}$. RTK se ha aplicado con éxito en Conducción autónoma [42] y agricultura de precisión [43]. 
El beneficio de la tecnología de posicionamiento para hacer vehículos más inteligentes, permite [4].

1. la implementación de Sistemas Avanzados de Asistencia al Conductor (ADAS) que pueden evitar colisiones.

2. reducción consumo de combustible.

3. es esencial un buen número de servicios, basados en ubicación, que hacen que la experiencia de conducción sea más placentera.

\subsection{DISEÑO SISTEMA DE PERCEPCIÓN DEL CICAR}

Como se demostrado en apartado 4.2 y se muestra en los gráficos de araña, no existe un sensor perfecto, por ello es necesario instalar múltiples sensores en el VA para detectar su entorno. Estos sensores miden diferentes magnitudes físicas, que normalmente se seleccionan para superponerse entre sí, proporcionando la información redundante necesaria para fusionar y correlacionar correctamente la información. Esto es necesario para obtener mejores estimaciones del entorno, ya que fusionar la información recopilada de diferentes sensores ayuda a reducir el número de interpretaciones plausibles de los datos medidos en entornos dinámicos y desconocidos. Por lo tanto, la selección de los tipos de sensores y su ubicación son claves para el diseño del sistema de percepción del VA.

La Tabla 4 muestra un resumen de características, modelo y configuraciones del sistema de percepción del CICar

\begin{tabular}{|c|c|c|}
\hline Modelo sensor & $\begin{array}{l}\text { Caraterísticas y } \\
\text { Configuraciones }\end{array}$ & Situación \\
\hline SICK LASER 2D TIM551 & 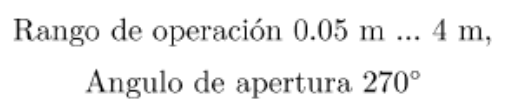 & Paragolpes delantero y trasero \\
\hline $\begin{array}{l}\text { LIDAR VELODYNE } \\
\text { HDL64 }\end{array}$ & $\begin{array}{c}120 \mathrm{~m} \text { range, } 1.3 \text { millones puntos } / \mathrm{s} \text {, } \\
26.9^{\circ} \text { Vertical FOV }\end{array}$ & En techo sobre baca metálica \\
\hline $\begin{array}{l}\text { Depth Camera Intel } \\
\text { RealSense D435 }\end{array}$ & $1280 \times 720$ y $1920 \times 1080$ RGB, $10 \mathrm{~m}$ & En techo sobre baca metálica \\
\hline DGPS & GPS asistido AHRS & En techo sobre baca metálica \\
\hline ToF Sentis3D-M420Kit & $\begin{array}{c}\text { Rango int } 7 \mathrm{~m} \text { Rango ext } 4 \mathrm{~m}, \\
\text { Horizontal FOV: } 90^{\circ}\end{array}$ & En techo sobre baca metálica \\
\hline $\begin{array}{l}\text { IMU Moog Crossbow } \\
\text { NAV440CA-202 }\end{array}$ & $\begin{array}{l}\text { Precisión pitch y roll }<0.4^{\circ} \\
\text { Posición }<0.3 \mathrm{~m}\end{array}$ & En techo sobre baca metálica \\
\hline EMLID RTK GNSS & Precisión posición $7 \mathrm{~mm}$ & En techo sobre baca metálica \\
\hline
\end{tabular}


En el diseño del sistema de percepción los sensores se divide en dos subsistemas: corto alcance (SRR) y largo alcance (LRR) como queda representado en la Figura 29.

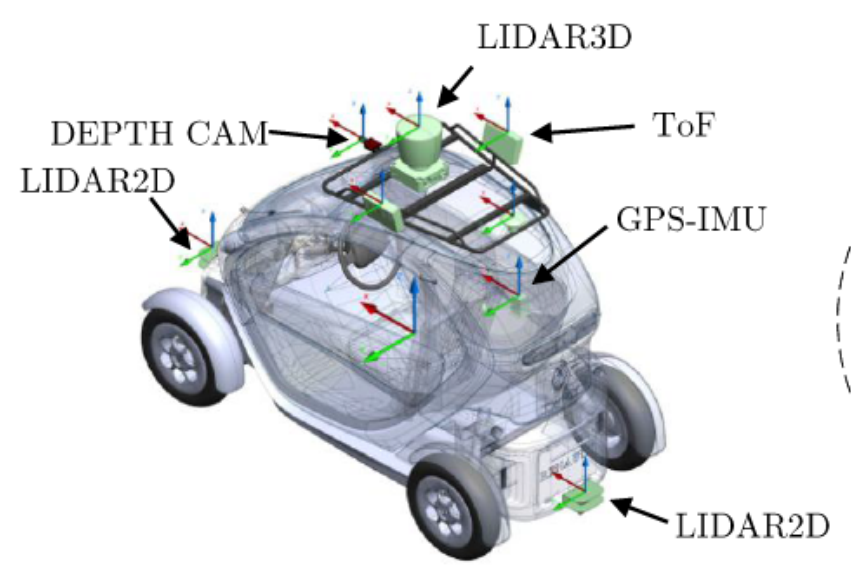

(a)

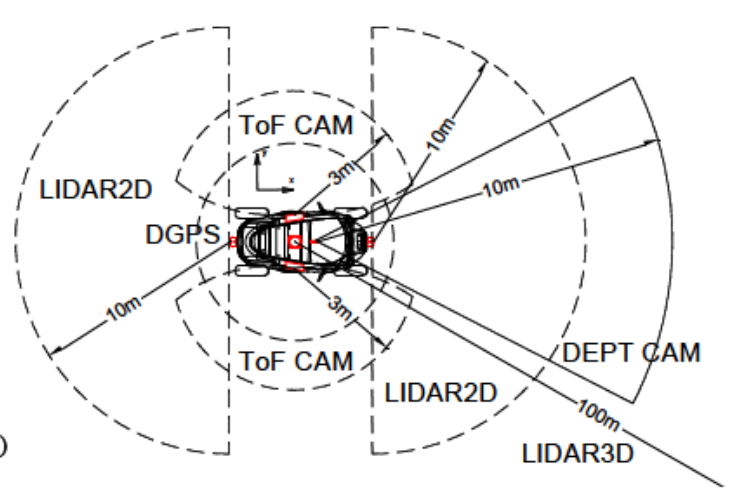

(b)

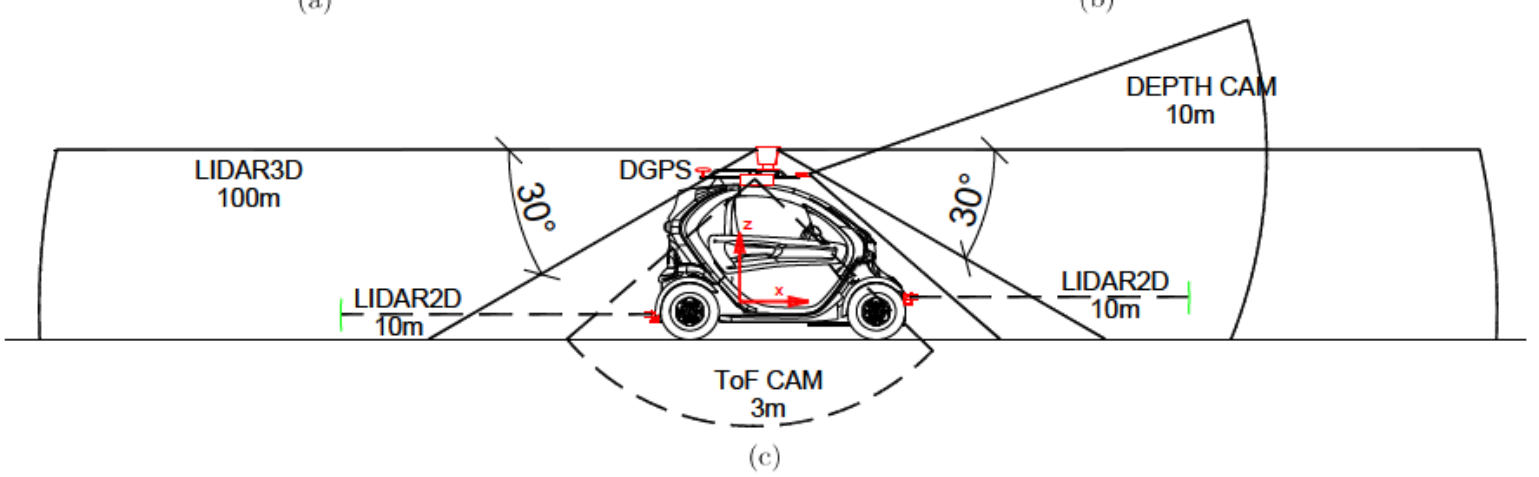

Figura 29. Vista de montaje de sensores

Un problema principal en el campo de la conducción autónoma es la incertidumbre del entorno exterior. Otro problema añadido es la cobertura espacial limitada. Se trata de instalar múltiples sensores, con cierta redundancia inherente, que permiten proporcionar la mayor información del entorno para mejorar la inferencia en localización y guiado. El fusionar la información reduce el conjunto de interpretaciones de entornos desconocidos y dinámicos. La información obtenida por estos sensores puede emplearse para aplicaciones como construcción de mapas, detección y monitorización, vigilancia, etc.

Los vehículos se convierten cada vez más inteligentes [4]. Son capaces de:

- Tomar información sobre su estado y / o el medio ambiente (más o menos distantes).

- Procesar información, procedente de los sensores a bordo, recopilar datos para tomar decisiones y pueden anticipar sus acciones.

- Además detectan situaciones que pueden ser peligrosas y mejoran la respuesta dinámica.

- La recopilación de información puede venir de varias maneras:

- Sensores a bordo en el vehículo que proporcionan información del exterior, que vigilan el entorno cercano, para los que existen diferentes tecnologías para corto y largo alcance, Figura 30. La tendencia propone el uso coordinado de varios sensores y la aplicación técnicas de fusión de sensores que permiten superar las limitaciones de cada sistema individualmente. 
- Posicionamiento del vehículo en un mapa digital. Esta función se lleva a cabo durante muchos años, la precisión en el posicionamiento y detalle de los mapas han ido creciendo con las especificaciones de los sistemas que se están incorporando en los vehículos.

- Recepción de información a distancia a través de comunicaciones inalámbricas. Estos datos tienen mayores posibilidades al ampliar el horizonte más allá de la visión física del vehículo. El potencial de esta información es la integración de grandes volúmenes de datos de diversas fuentes.

- Vigilancia del conductor y del interior del vehículo. Se requieren otro tipo de algoritmos debido a que las variables que se buscan son completamente diferentes.

La Tabla 5 muestra las propiedades principales de diferentes tecnologías de detección comúnmente utilizadas en el vehículo para la conducción autónoma [44].

\begin{tabular}{ccccccc}
\hline & $\begin{array}{c}\text { Rango } \\
\text { óptimo } \\
\text { operación }\end{array}$ & info & Ratio & $\begin{array}{c}\text { Medida de } \\
\text { velocidad }\end{array}$ & $\begin{array}{c}\text { Funcionamiento } \\
\text { con lluvia }\end{array}$ & $\begin{array}{c}\text { Funcionamiento } \\
\text { de noche }\end{array}$ \\
\hline Vision & $0-25 \mathrm{~m}$ & $\begin{array}{c}\text { 2D / } \\
\text { 3D }\end{array}$ & High & No & Bad & Weak \\
$\begin{array}{c}\text { Radar } \\
\text { LIDAR } \\
\text { 2D }\end{array}$ & $1-200 \mathrm{~m}$ & ----- & High & Yes & Excellent & Excellent \\
$\begin{array}{c}\text { LIDAR } \\
\text { 3D }\end{array}$ & $1-20 \mathrm{~m}$ & 2D & Medium & No & Weak & Excellent \\
\hline
\end{tabular}

Tabla 5. Propiedades de diferentes tecnologías de detección

Entre los sensores incorporados en un vehículo autónomo, se pueden distinguir sensores de corto y largo alcance, Figura 30. En el primer caso, se pueden citar sensores ultrasónicos, infrarrojos y capacitivos. Entre el segundo grupo, los LIDAR 2D, LIDAR 3D y las tecnologías de visión por ordenador parecen ofrecer el mejor rendimiento.

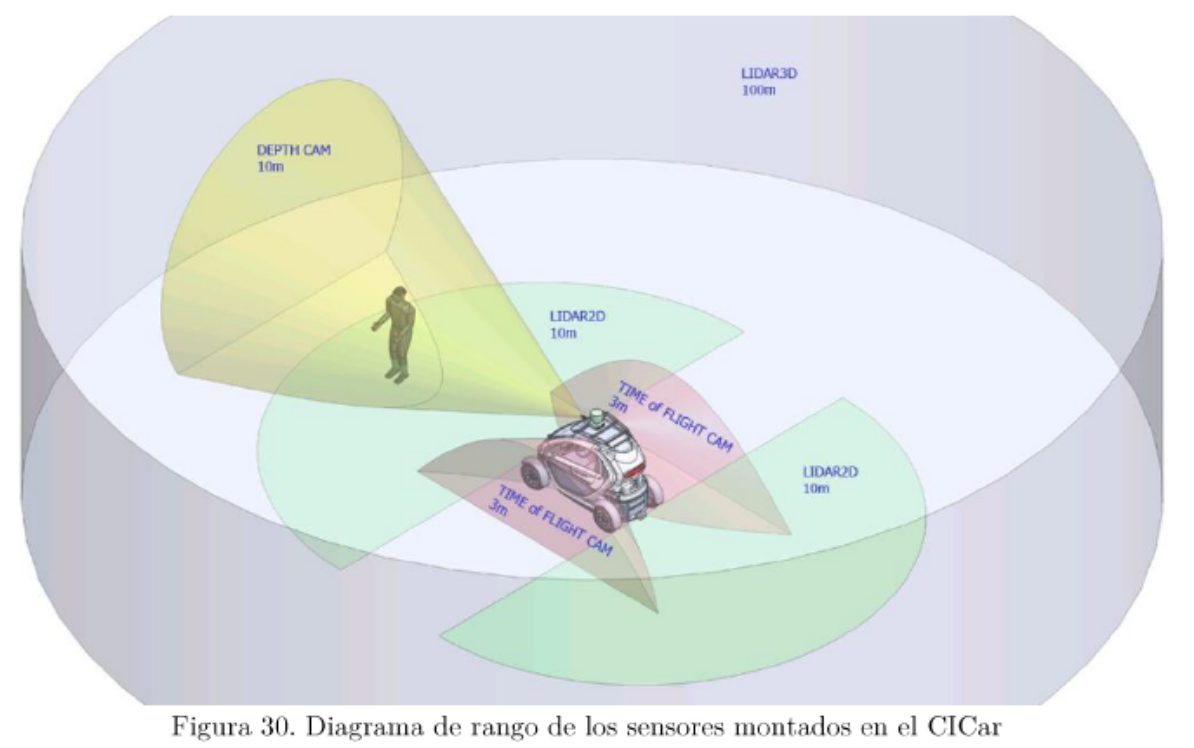




\subsubsection{SISTEMA PERCEPCEPCIÓN DE CORTO ALCANCE (SRR)}

Se considera de corto alcance, son adecuados para numerosas áreas de aplicación alrededor del vehículo. Tienen diferentes principios de operación, dependiendo de la distancia que se desea medir. Usan un método por triangulación para mayor precisión de medición de distancia en rangos cortos, mientras que para rangos largos prefieren el método óptico de tiempo de vuelo (ToF). La medición de la distancia es en gran medida independiente del color objetivo o del tipo de superficie y la repetibilidad es alta.

El SRR permite detectar objetos hasta 10 metros desde la parte delantera y trasera del vehículo (LIDAR 2D) y alrededor de 3 metros desde el lado derecho e izquierdo del vehículo (cámaras ToF). Las cámaras SENTIS ToF son una tecnología poco utilizada en vehículos autónomos, pero resultan ser una solución muy atractiva para la detección de corto alcance para el vehículo. Los ToFs permiten la detección de objetos 3D con una alta velocidad de cuadros, se ven menos afectados por las condiciones de luz y las sombras y tienen un costo aceptable [45] [46]. Los objetos que entran en el anillo de corto alcance, suponen un riesgo inherente y peligro para las acciones del CICar. Por esta razón, los sensores involucrados en el SRR son ordenados por la unidad de procesamiento RT.

Estos sistemas detectan entornos cercanos que los sensores de larga alcance, no pueden visualizar. Son por ejemplo bordillos de aceras, marcas de carretera, obstáculos y personas o animales que repentinamente aparecen en el camino próximo del vehículo, incluso aéreas libres de objetos.

\begin{tabular}{cccc}
\hline SENTIS ToF & Láser 2D delantero & Láser 2D trasero & Cámara frontal \\
\hline
\end{tabular}

Tabla 6. Sistemas de corto alcance montados en el CICar

\section{SENSORES DE PROFUNDIDAD TOF}

Este sensor 3D, figura 28, tiene como principio de funcionamiento el denominado "tiempo de vuelo" (ToF). Una fuente de infrarrojos emite luz que rebota en el cuerpo y vuelve a un sensor. Por el tiempo que transcurre, se calcula la distancia a la que se encuentran los diferentes objetos. La información en 3D se codifica por colores: el rojo representa lo más cercano; el azul, lo más lejano. Usando la iluminación activa del IR, el sensor puede capturar imágenes en una resolución de $160 \times 120$ pixeles con hasta 40 secuencias por segundo independientemente de luz ambiente. Es posible analizar las escenas basadas en los datos 3D solamente o conjuntamente con los datos del grayscale. El vehículo lleva instalados dos unidades a cada lado. Visualiza el entorno lateral cercano, que algunos sensores no pueden ver.

\section{LIDAR 2D}

Este láser 2D obtiene una segmentación y caracterización del entorno a través de lecturas obtenidas por un barrido en plano horizontal. El vehículo lleva montado dos unidades, una en la parte frontal y otro en la parte trasera. 


\section{CÁMARA DE VISIÓN FRONTAL}

La cámara de visión frontal es una solución de seguimiento estéreo que ofrece profundidad de calidad para una variedad de aplicaciones, mezcla ToF y imágenes en el espectro visible. Posee amplio campo de visión adecuado para la realidad virtual y aumentada del entorno del vehículo. Con un alcance de hasta $10 \mathrm{~m}$, esta cámara de factor de forma pequeño se integra con facilidad en la vaca del vehículo.

La combinación de un módulo Intel y un procesador de visión juntos en un factor de forma pequeño produce una solución ideal para el desarrollo como para la creación rápida de productos. Posee un software altamente personalizable, proporciona una solución de bajo costo que es liviana y poderosa, lo que permite el desarrollo de soluciones de detección de próxima generación que pueden comprender e interactuar con su entorno.

La combinación de un amplio campo de visión y un sensor de obturador global en la cámara frontal, denominada D435 la convierte en la solución preferida para aplicaciones como la navegación robótica y el reconocimiento de objetos. Los sensores de obturador globales proporcionan una gran sensibilidad con poca luz, lo que permite al vehículo navegar por los espacios con las luces apagadas.

Incorpora el último hardware y software de detección de profundidad de Intel, los empaqueta en productos fáciles de integrar.

\subsubsection{SISTEMA PERCEPCEPCIÓN DE LARGO ALCANCE (LRR)}

La percepción de LRR se basa en un LIDAR de alta definición en 3D (HDL64SE de Velodyne) [47]. Sus 64 rayos láser giran a $800 \mathrm{rpm}$ y pueden detectar objetos a una distancia de hasta 100 metros con una precisión de $2 \mathrm{~cm}$. El LIDAR 3D permite establecer trayectorias (cambio de carril, evitar obstáculos, reducir la velocidad según las condiciones del tráfico, etc.), seguimiento de objetos, clasificación de objetos y predicción de comportamiento de otros conductores. La información de los dos subsistemas se fusiona y procesa para detectar obstáculos como: automóviles, bicicletas, peatones, semáforos, etc. Una cámara a color se coloca en el techo del vehículo y se usa para detectar: la carretera, los carriles, las vías y las señales de tráfico.

Es imprescindible, tener una visión de entorno más allá del contorno del vehículo. Lógicamente cuanto más información de los alrededores relativamente lejanos, mejor anticipación de movimientos y acciones tendrá el vehículo. Estos sistemas de largo alcance, por norma general, interpretan escenarios y obstáculos fijos, estáticos, que en cierto tiempo se encontrarán o pasarán a pocos metros del vehículo.

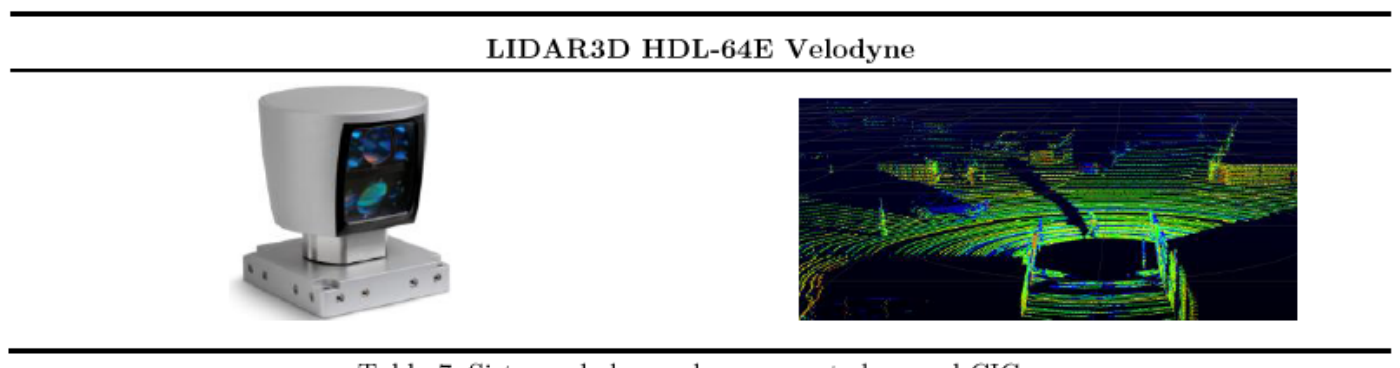

Tabla 7. Sistema de largo alcance montados en el CICar 
Las reconstrucciones virtuales realizadas con sensores LIDAR 3D [48][49], están siendo utilizadas para resolver problemas en una amplia gama de sectores, que obtienen modelos 3D de ciudades que pueden resolver problemas de tráfico, detectar y prevenir desastres en minas, obtener la topología y cartografía de la tierra para diseñar el crecimiento organizado de las ciudades.

Este sensor de largo rango, LIDAR Velodyne HDL-64E está diseñado para la detección de obstáculos y de navegación de vehículos terrestres autónomos. Su campo de visión es de $360^{\circ}$, con alta velocidad de datos hace que este sensor sea ideal para las aplicaciones exigentes de percepción, así como aplicaciones de captura de datos móviles y cartografía 3D.

Con la ayuda de las herramientas de software, la nube de puntos escaneados y los objetos detectados se pueden usar para crear un modelo del entorno del vehículo y para calcular varias variables dinámicas individuales para apoyar la navegación.

\subsubsection{SISTEMAS DE POSICIONAMIENTO PARA VAS}

El CICar tiene un sistema de localización basado en una unidad RTK (Cinemática en tiempo real), un IMU (Unidad de medición inercial) y un codificador de posición relativa instalado en la rueda del vehículo. La navegación por satélite RTK consiste en una técnica utilizada para mejorar la precisión de los datos de posición derivados de los sistemas de posicionamiento basados en satélites (sistemas de navegación global por satélite, GNSS) como GPS, GLONASS, Galileo y BeiDou. RTK se compone de una estación base y una unidad móvil (o móvil), que proporciona una precisión de $20 \mathrm{~cm}$ a $1 \mathrm{~cm}$. El sistema de localización cancela el posicionamiento RTK cuando el número de satélites detectado es menor o igual a cuatro o cuando el HDOP (Dilución de precisión horizontal) es mayor que cinco [50]. En estos casos, la IMU y el codificador de posicionamiento instalados en el vehículo se utilizan para la tarea de localización.

\section{UNIDAD DE MEDIDA INERCIAL (IMU)}

Dead-Reckoning DR es el proceso de estimar la posición y el rumbo de un vehículo según la posición anterior medida. Las estimaciones de posición más simples se realizan utilizando sensores rotativos fijados al volante y las ruedas del vehículo [51], una técnica conocida como odometría que no es capaz de cuantificar los deslizamientos o movimientos laterales del vehículo. Por este motivo se instalan unidades de medida inerciales (IMU) que combinan acelerómetros, giroscopios y Magnetómetros. La incorporación de estos sensores permite que los errores mencionados anteriormente sean corregidos y la velocidad de muestreo del sistema de medición se puede aumentar. Sin embargo, ellos pueden introducir errores debidos a la medición de las variables de primer y segundo orden [4]. Los IMU no solo proporcionan posición global del vehículo, generalmente se acompañan con un sistema GNSS. Los sistemas de posicionamiento actuales para vehículos son sistemas híbridos que combinan datos de diferentes fuentes como odometría, IMU, GNSS, LIDAR, RADAR y cámaras para obtener una posición confiable y rumbo con error tolerable.

Un IMU es en general un sistema cerrado que es usado para detectar la orientación, localización y movimiento. Normalmente este dispositivo usa una combinación de acelerómetros y sensores de velocidad angular (giróscopos) para conocer cómo se está moviendo éste y en qué posición se encuentra.

El término IMU está ampliamente extendido, contiene 3 acelerómetros. Estos están situados de tal forma que sus ejes de medida son mutuamente ortogonales. Miden las llamadas "fuerzas específicas" (aceleración inercial - gravedad). 
Proporciona información sobre la orientación, aceleraciones lineales y velocidades angulares en los tres ejes del espacio, a través de giróscopos, magnetómetros y acelerómetros

Como ahora veremos, en las transformaciones lineales en el espacio 3D habitualmente se utilizan sistemas de coordenadas homogéneos, ya que en la mayoría de los casos es aconsejable la composición matricial.
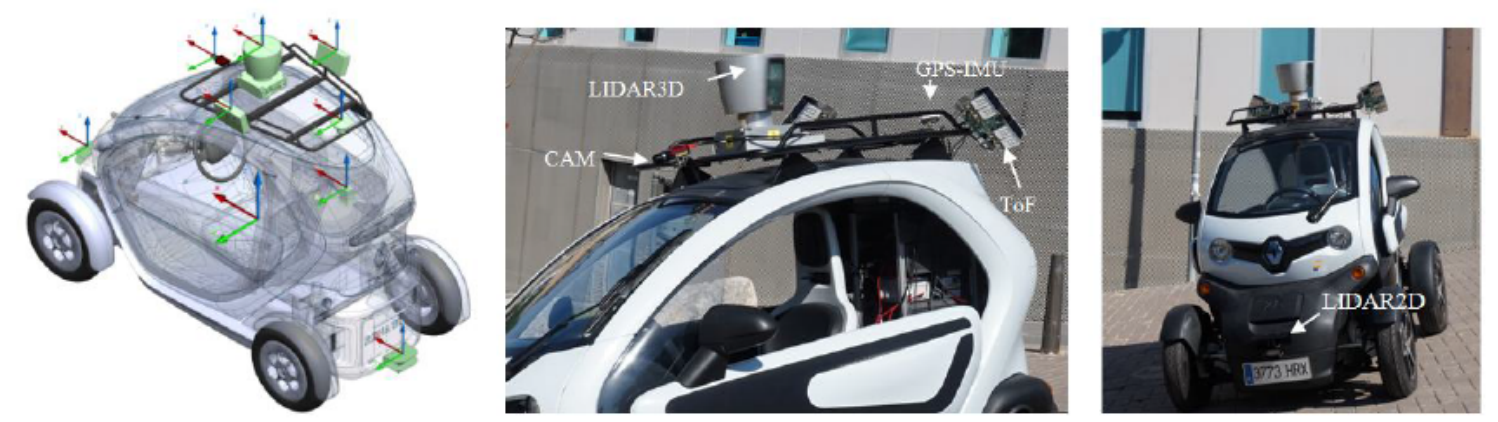

Figura 31. Sistemas de referencia de sensores en el vehículo autónomo

\begin{tabular}{|c|c|c|c|c|c|c|}
\hline $\begin{array}{c}\text { SENTIS ToF } \\
\text { derecho }\end{array}$ & $\begin{array}{c}\text { SENTIS ToF } \\
\text { izquierdo }\end{array}$ & $\begin{array}{l}\text { LIDAR 2D } \\
\text { delantero } \\
\end{array}$ & $\begin{array}{c}\text { LIDAR 2D } \\
\text { trasero } \\
\end{array}$ & $\begin{array}{l}\text { Cámara } \\
\text { frontal } \\
\end{array}$ & IMU & LIDAR 3D \\
\hline X 0.30 & X 0.30 & X 1.30 & $\mathrm{X}-1.30$ & X 0.80 & X 0.40 & X 0.40 \\
\hline Y 0.65 & $\mathrm{Y}-0.65$ & Y 0.00 & Y 0.00 & Y 0.00 & Y 0.00 & Y 0.00 \\
\hline Z 1.05 & Z 1.05 & $\mathrm{Z}-0.25$ & $\mathrm{Z}-0.25$ & $\mathrm{Z} 0.55$ & Z 1.05 & Z 1.15 \\
\hline
\end{tabular}

Tabla 8. Distancias ejes de referencia sistema percepción CICar

La translación de un objeto consiste en moverlo cierta distancia, en una dirección determinada.

En 3D, el sistema de referencia homogéneo tendrá 4 dimensiones, por lo que la traslación del punto $\mathrm{V}=(\mathrm{x}, \mathrm{y}, \mathrm{z}, 1)$ quedará indicada como

$$
\mathrm{V}^{\prime}=\left(\mathrm{x}^{\prime}, \mathrm{y}^{\prime}, \mathrm{z}^{\prime}, 1\right)=(\mathrm{x}, \mathrm{y}, \mathrm{z}, 1) \cdot \mathrm{T}
$$

siendo T la matriz de traslación 3D.

$$
\mathbf{T}=\left[\begin{array}{cccc}
1 & 0 & 0 & 0 \\
0 & 1 & 0 & 0 \\
0 & 0 & 1 & 0 \\
t_{x} & t_{y} & t_{z} & 1
\end{array}\right]
$$

$(\mathrm{tx}, \mathrm{ty}, \mathrm{tz})$ se conoce como el vector de traslación.

La expresión anterior es equivalente al sistema de ecuaciones

$$
\begin{aligned}
& x^{\prime}=x+t_{x} \\
& y^{\prime}=y+t_{y} \\
& z^{\prime}=z+t_{z} \\
& 1=0+1
\end{aligned}
$$

Como se comentó arriba, para trasladar un objeto poliédrico se ha de aplicar la matriz $\mathrm{T}$ a todos los vértices del objeto. Es importante observar que al hacer la traslación de un objeto sus proporciones no varían, puesto que todos los puntos significativos (vértices en el caso de los poliedros) se mueven la misma distancia, en la misma dirección.

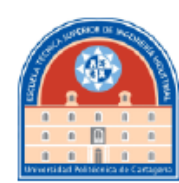




\subsection{FILTRO DE KALMAN}

El filtro de Kalman [52] permite la fusión de datos estadísticamente óptimos de los datos de posicionamiento absoluto provenientes de GNSS con datos para el posicionamiento relativo provenientes de sensores en el vehículo. Haciendo uso del Filtro de Kalman, la precisión de posicionamiento puede mejorarse.

\subsubsection{VARIABLES Y PARÁMETROS DEL FILTRO DE KALMAN}

Existe la posibilidad de diseñar el filtro, matemáticamente hablando, de dos formas; con ecuaciones normales, cuando hablamos de sistemas simples de un sensor y una variable o con ecuaciones matriciales cuando trabajamos con sistemas más complejos. Se representarán las variables y parámetros en letra mayúscula para hablar en forma matricial aunque es perfectamente aplicable a sistemas no matriciales [53].

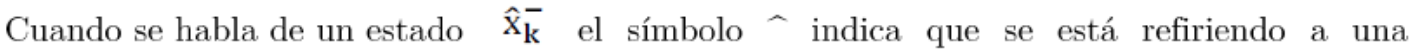
estimación, no al valor real. El superíndice - indicará que se habla de una variable a priori, es decir, de una predicción antes de ser corregida. Cuando se hable acerca de una variable a posteriori no se usará superíndice. Para finalizar, el subíndice $\mathrm{k}$ indica que la variable es referida a la iteración k, por tanto al hablar de k-1 se está haciendo referencia al valor en la iteración anterior.

A continuación se describirán las variables y parámetros que aparecen en las ecuaciones del filtro:

$\mathrm{x}_{\mathrm{k}}$ es vector de variables del estado del sistema. La estimación de dichas variables es el resultado del proceso de filtrado.

$\mathrm{z}_{\mathrm{k}}$ es vector de mediciones aportadas por los sensores del sistema.

$\mathrm{u}_{\mathrm{k}}$ es vector de señales de control del sistema.

$\mathrm{K}_{\mathrm{k}}$ es la matriz de ganancias de Kalman. Determinará el peso de cada variable en el proceso de fusión.

$\mathrm{v}_{\mathrm{k}}$ es ruido de medición.

$\mathrm{w}_{\mathrm{k}}$ es ruido del proceso de filtrado, referido al error del modelo.

A es matriz de transición de estado.

B es matriz de coeficientes que escalan $u_{x}$.

$\mathrm{H}$ es matriz de coeficientes que escalan $\mathrm{x}_{\mathrm{x}}$.

$\mathrm{R}$ es matriz de covarianza del ruido de las mediciones.

Q es matriz de covarianza del ruido del proceso.

$\mathrm{P}_{\mathrm{k}}$ es matriz de covarianza de error en la estimación del estado.

I es matriz identidad. En sistemas no matriciales se sustituye por 1 .

Las matrices de covarianza $\mathrm{R}$ y $\mathrm{Q}$ son definidas por el usuario teniendo la siguiente forma:

$$
R=\left[\begin{array}{cc}
\sigma_{z 1}^{2} & \sigma_{z 1, z 2} \\
\sigma_{z 1, z 2} & \sigma_{z 2}^{2}
\end{array}\right]_{n x n} \quad Q=\left[\begin{array}{cc}
\sigma_{x 1}^{2} & \sigma_{x 1, x 2} \\
\sigma_{x 1, x 2} & \sigma_{x 2}^{2}
\end{array}\right]_{m x m}
$$


La dimensión $\mathrm{n}$ es igual a la dimensión del vector de mediciones z, mientras que $\mathrm{m}$ hace referencia a la dimensión del vector de estado x. Se entiende por $\sigma_{z 1}^{2}$ la varianza asociada al sensor z1, mientras que $\sigma_{z 1, z 2}^{2}$,representa la covarianza de los datos de sensores z1 y z2. Análogamente $\sigma_{x 1}^{2}$ es la varianza asociada a la variable x1 y $\sigma_{x 1, x 2}^{2}$, la covarianza de los estados 1 y 2 del vector x. Las componentes de $\mathrm{R}$ pueden ser calculadas mientras que para Q será necesaria la estimación unos valores de los que dependerá la eficacia del filtro.

\subsubsection{MODELO Y ECUACIONES}

Para la aplicación del filtro se parte de un modelo del sistema representado según las siguientes ecuaciones:

$$
\begin{gathered}
\mathrm{x}_{k}=\mathrm{Ax}_{k-1}+B u_{k}+w_{k-1} \\
z_{k}=H \mathrm{x}_{k}+\mathrm{v}_{k}
\end{gathered}
$$

La primera ecuación representa las variables evaluadas como una variación de las mismas en el estado anterior, teniendo en cuenta los cambios producidos por la señal de control y el ruido del sistema.

La segunda ecuación describe las observaciones de los sensores como una función de las variables objetivo, sumando una componente de ruido de medición. Una de las variables reales $\mathrm{x}$ puede no ser medida directamente por un sensor, pero sí obtenida por una ecuación matemática lineal, por ello se emplea la matriz $\mathrm{H}$, que relaciona las variables evaluadas con los datos observables.

Una vez presentado el modelo se procede a la explicación de las ecuaciones del filtro.

Como se comentó anteriormente existen dos grupos de ecuaciones; ecuaciones de predicción y ecuaciones de actualización. Las primeras realizan una predicción a partir del estado anterior y los datos del modelo, mientras que las segundas aplican una corrección a dicha predicción teniendo en cuenta los datos procedentes de los sensores.

Ecuaciones de predicción:

$$
\begin{aligned}
& \hat{x}_{k}^{-}=\mathrm{A} \hat{x}_{k-1}+B u_{k} \\
& P_{k}^{-}=A P_{k-1} A^{T}+Q
\end{aligned}
$$


Ecuaciones de corrección:

$$
\begin{gathered}
K_{k}=P_{k}^{-} H^{T}\left(H P_{k}^{-} H^{T}+R\right)^{-1} \\
\hat{\mathrm{x}}_{k}=\hat{x}_{k}^{-}+K_{k}\left(z_{k}-H \hat{x}_{k}^{-}\right) \\
P_{k}=\left(I-K_{k} H\right) P_{k}^{-}
\end{gathered}
$$

La ecuación (34) realiza una predicción del estado evaluado en función de la estimación de la iteración anterior. La ecuación (34) representa la ecuación del modelo (32) sin el término de ruido, convirtiendo por tanto todas las variables en estimaciones. (35) realiza una predicción recursiva del error teniendo en cuenta la matriz de covarianza de error del proceso Q.

Para poder explicar adecuadamente las ecuaciones de corrección, se procede a evaluar la tendencia de la ecuación (37) en función de los valores que puede alcanzar la ganancia K. Para simplificar la explicación se recurre a las ecuaciones en forma no matricial:

$$
\hat{\mathrm{x}}_{k}=\hat{x}_{k}^{-}+k_{k}\left(z_{k}-\hat{x}_{k}^{-}\right)
$$

Partiendo del supuesto que la ganancia $\mathrm{k}_{\mathrm{k}}$ tiene un valor de 0 , la ecuación (39) conduce a

$$
\hat{\mathrm{x}}_{k}=\hat{x}_{k}^{-}
$$

Asignando a la ganancia $\mathrm{k}_{\mathrm{k}}$ un valor de 1, en (39) se obtiene:

$$
\hat{\mathrm{x}}_{k}=z_{k}
$$

Se puede concluir por tanto que la ganancia de Kalman distribuye en (37) el peso de la predicción y los datos observados en función de la ganancia K, la cual es calculada en (36) a partir del error en P y en R. Finalmente la ecuación (38) corrige el error a partir de la ganancia.

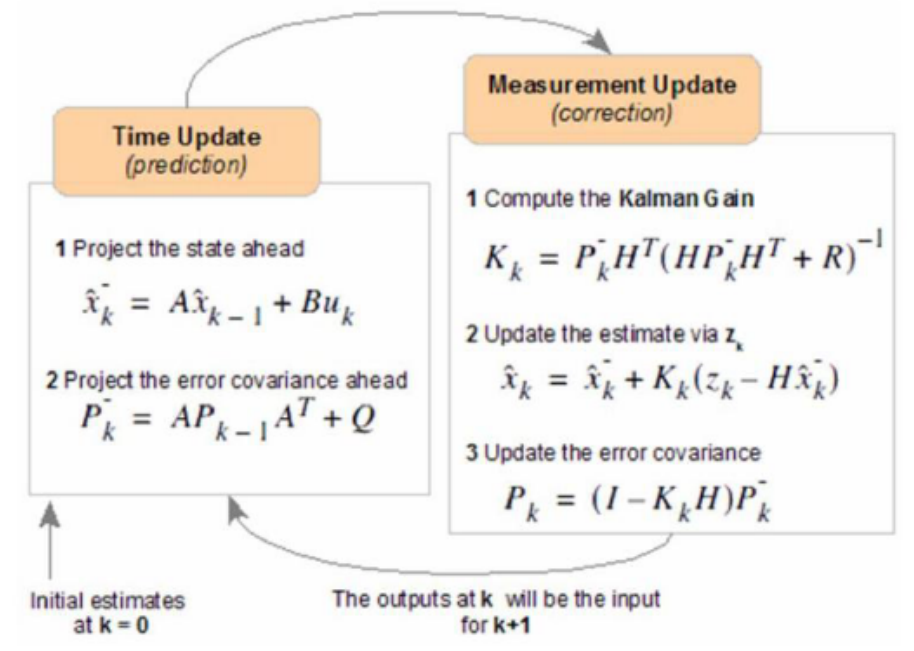

Figura 32. Funcionamiento del filtro de Kalman 
Como puede verse en la Figura 32, para la inicialización del filtro son necesarias unas estimaciones iniciales $\mathrm{x}_{0}$ y $\mathrm{P}_{0}$ que iniciarán el proceso iterativo cuya estructura comienza con las ecuaciones de predicción y termina con las ecuaciones de corrección para continuar con la siguiente iteración. 


\section{CAPITULO 5}

\section{SISTEMA DE TOMA DE DECISION}

\subsection{INTRODUCCION}

La capacidad de conducción humana requiere no sólo la capacidad de maniobrar adecuadamente el volante, el freno, el acelerador y dirección de marcha de acuerdo con un conjunto de normas de tráfico, sino también evaluar los riesgos sociales, la salud, las consecuencias legales o la duración de las acciones de conducción (por ejemplo, si un peatón no se detiene en una luz roja). La resolución de la conducción requiere altos niveles de conocimiento humano, por esta razón la ciencia utiliza complejos sistemas para emularlos. Los sistemas de toma de decisiones tienen el papel de interpretar el suministro de información abstracta desde el sistema de percepción del vehículo y generar acciones para llevar a cabo una conducción sostenible y de seguridad.

La toma de decisiones críticas es la clave para la autonomía y se realiza a través de algoritmos de planificación, incorporados en el middleware del módulo de navegación, comprensión del entorno, ejecución de acciones sobre el vehículo autónomo, etc. [54]. Estos algoritmos reciben información del sistema de percepción junto con entradas de mapas digitales para proporcionar un espacio de trabajo completo en el que se lleva a cabo la planificación.

El objetivo principal de la planificación es proporcionar al vehículo un camino seguro y sin colisiones hacia su destino, teniendo en cuenta la dinámica del vehículo, sus capacidades de maniobra en presencia de obstáculos, junto con las normas de tránsito y los límites de las carreteras [55]. La planificación es una rutina que consume memoria, así como una rutina de computación intensiva, que se ejecuta en paralelo con otras operaciones rutinarias del vehículo (por ejemplo, seguimiento de obstáculos, fusión de datos y módulos de control). Las entradas y salidas de una planificación de movimiento normalmente dependen de estos otros módulos. La planificación confiable, robusta y adaptable es esencial, especialmente en un escenario de tráfico urbano mixto.

En este capítulo se muestra una clasificación de los sistemas de toma de decisión compuesta por (1) sistemas de toma decisión tradicional, (2) sistemas inteligentes de toma decisión.

Lograr una conducción autónoma de alta fidelidad requiere probar las características autónomas en todos los escenarios posibles. El diseño, la implementación y las pruebas de vehículos en una amplia gama de casos de uso y en condiciones de tráfico realistas son costosos, requieren mucho tiempo, son complicados y, a menudo, no se pueden reproducir. La integración de pruebas con la plataforma física en estos casos son innecesariamente complejas y muchas veces se realizan en la última etapa del proceso de desarrollo. Esto hace que el diseño, implementación, pruebas intensivas y simulación de prototipos con un vehículo real en el circuito de simulación sea la forma más efectiva de verificar y validar la idea de diseño.

Se describe al final del capítulo la arquitectura propuesta, como sistema de decisión basada en un sistema tradicional formado por planificador global PPS y planificador local PPL. 


\subsection{SISTEMAS DE TOMA DECISIÓN TRADICIONAL}

Para operar de forma fiable en el mundo real, un vehículo autónomo debe evaluar y tomar decisiones sobre las consecuencias de sus posibles acciones anticipando las intenciones de otros participantes en el tráfico. Los primeros sistemas de toma de decisiones en vehículos autónomos aparecen en 2007 en DARPA Urban Challenge [56]. Estos sistemas permitieron que los vehículos funcionaran en escenarios urbanos en los que estuvieran involucrados, giros U, intersección, áreas de estacionamiento y tráfico real entre otros. Estos primeros sistemas de decisión usaban elementos comunes como planificadores cuyos sistemas se implementaban usando máquinas de estados finitos, árboles de decisión y heurísticas.

Particularmente la Figura 33 muestra una arquitectura común de un sistema de decisión tradicional formado por un sistema de percepción, un planificador de ruta, un componente de conducta de conducción, un planificador de movimiento y por último una interface con el vehículo

El sistema de percepción es el encargado de localizar adecuadamente la posición del vehículo y percibir su entorno [57]. Las funciones que realiza el sistema de percepción de estos vehículos son: (1) detección y clasificación de obstáculos, (2) localización que incluye la posición y orientación del vehículo, (3) detección de la vía, (4) ubicación en mapa digital, (5) análisis dinámico de obstáculos.

El sistema de planificación utiliza un modelo híbrido deliberativo-reactivo que divide las decisiones de nivel superior y reacciones de nivel inferior en componentes separados. Estos componentes se ejecutan simultáneamente, lo que permite que el vehículo reaccione a situaciones de emergencia sin necesidad de volver a planificar toda la ruta.

El planificador de ruta es responsable de determinar las trayectorias y zonas que el vehículo puede utilizar para alcanzar todos los puntos de control.

En un nivel de abstracción superior, el componente conducta de conducción es el encargado de seguir las reglas de conducción y guiado al vehículo por la ruta planificada.

El último elemento del sistema de decisión tradicional está compuesto por un interface con el vehículo que permite llevar a cabo las acciones elementales de conducción (giro de volante, acción de freno, acelerador y sentido de la marcha).

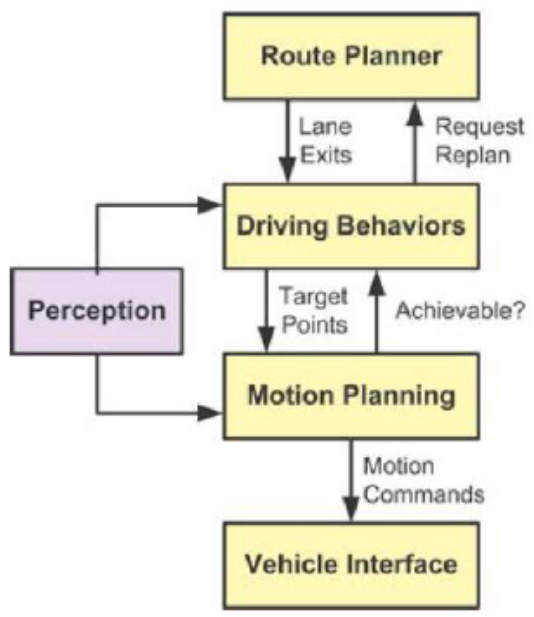

Figura 33. Esquema sistema de toma decisión tradicional [57] 


\subsection{SISTEMAS INTELIGENTES DE TOMA DE DECISIÓN}

Los enfoques más recientes han abordado el problema de la toma de decisiones para la conducción autónoma a través de la lente de la optimización de la trayectoria. Sin embargo, estos métodos no modelan las interacciones de bucle cerrado entre los vehículos, por no razonar sobre sus resultados potenciales [58]. Hoy en día, no hay sistemas reales que superan a un conductor humano. Los avances en la toma de decisiones tienen como objetivo aumentar la inteligencia de los sistemas involucrados en la toma de decisiones. Los sistemas cognitivos [59], los sistemas de agentes [60], los sistemas difusos [61], las redes neuronales [62], algoritmos evolutivos [63] o los métodos basados en reglas [64] componen los Intelligent Decision Making Systems (IDMS).

La Figura 34 muestra la ubicación de IDMS desde el punto de vista de la arquitectura funcional para la conducción autónoma [65].

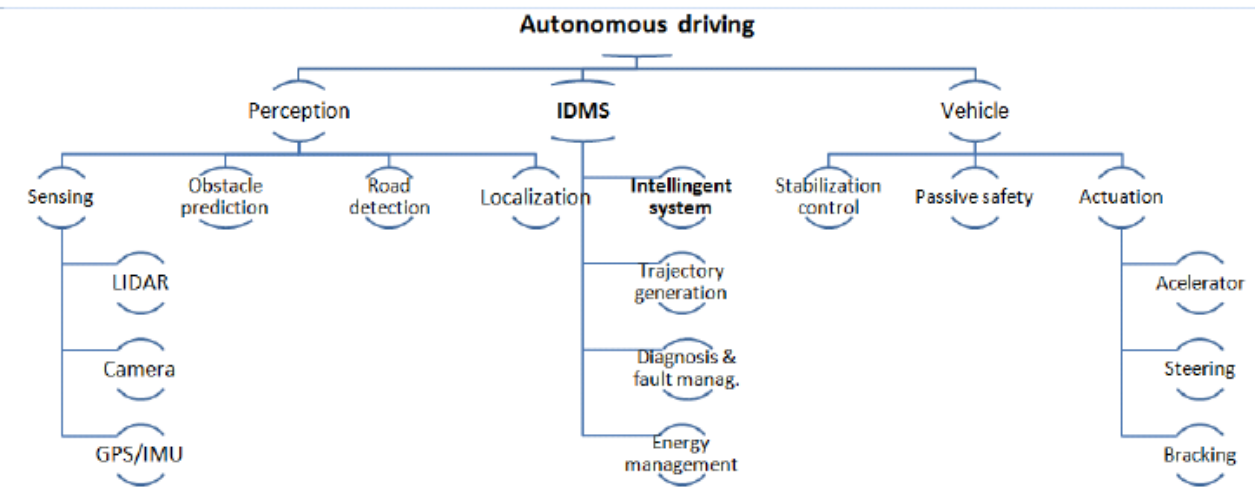

Figura 34. Arquitectura funcional de conducción autónoma

En general la arquitectura de un IDMS contendrá una unidad de procesamiento sensorial, un modelo del mundo, un generador de comportamiento y un componente de juicio de valor como muestra la Figura 35.

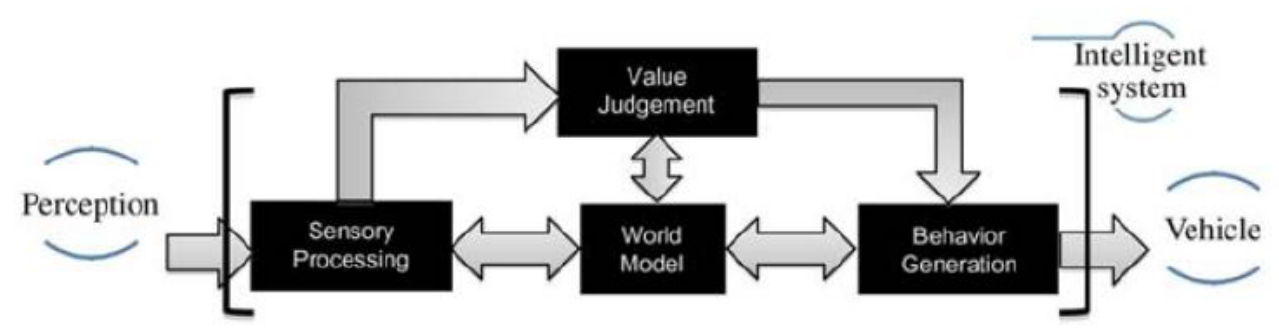

Figura 35. Descripción general del sistema inteligente ANSI

La conducción autónoma en escenarios complejos donde múltiples vehículos están involucrados (por ejemplo, áreas urbanas) con un riesgo inherente a la integridad de las personas, requieren soluciones en tiempo real.

En este caso, la toma de decisiones exige confiabilidad, seguridad y un sistema de tolerancia a errores. En esta dirección el enfoque por [66] utiliza un algoritmo de planificación en tiempo real basado un árbol de exploración aleatoria (RRT). El algoritmo propuesto demostró la capacidad de completar una misión militar, mientras interactuaba de manera segura con otros vehículos autónomos y humanos. 
Otra método para solucionar el problema de decisión en tiempo real está basado en toma de decisiones multicriterio MCDM; [67] basadas en redes de Petri [68]. Las MCDM ofrece una variedad de beneficios tales como:

- La jerarquía de objetivos permite una especificación sistemática y completa de objetivos a alcanzar por el vehículo.

- Las funciones de utilidad pueden definirse de manera heurística para reflejar las elecciones de un controlador humano, o alternativamente, se pueden aplicar algoritmos de aprendizaje.

- MCDM permite la integración y evaluación de un gran número de alternativas de conducción.

- La flexibilidad de la decisión se puede lograr definiendo el conjunto de atributos importantes dependiendo de las condiciones de la carretera.

- Se pueden agregar objetivos, atributos y alternativas adicionales sin la necesidad de cambios importantes.

- Las maniobras de conducción se modelan como autómatas finitos determinísticos.

- La unidad de toma de decisiones se modela como una red Petri.

El autor concluyó que el método estaba altamente basado en heurística, pero la aplicación de MCDM en esta nueva área de investigación ofrece una variedad de beneficios con respecto a la especificación del problema, flexibilidad de decisión y escalabilidad.

\subsection{SIMULACIÓN Y HERRAMIENTAS SOFTWARE PARA DMS}

El modelado y la simulación son herramientas bien establecidas para el análisis, el diseño, la adquisición y la formación en el ámbito de la automoción. A pesar de la heterogeneidad de subsistemas y disciplinas involucradas en el desarrollo de un vehículo autónomo, existen muchos métodos de simulación que en conjunto permiten cubrir todo el proceso de desarrollo.

Aunque el número de herramientas para la simulación de vehículos autónomos ha aumentado en los últimos años, es muy difícil seleccionar la mejor herramienta para un desarrollo específico. Características como: código abierto, multiplataforma, personalización y documentación son deseables en todos los simuladores. Además, con respecto a la simulación de dichos sistemas robóticos, se deben tener en cuenta todos los aspectos de la implementación física para simplificar aún más la transición de escenarios del mundo virtual al mundo real.

La mayoría de las herramientas de simulación son compatibles con lenguajes de programación como C / C ++, Perl, Python, Java, LabVIEW, URBI o MATLAB.

La simulación de Software-In-the-Loop (SIL) en el entorno de laboratorio ofrece una forma segura de prototipar e implementar el control y algoritmos de vehículos. La incorporación de un vehículo y sensores reales en el bucle de simulación (denominado Hardware-In-the-Loop o HIL) valida el diseño y reduce el tiempo necesario para la verificación del sistema. 
Estas herramientas de software proporcionan entornos, plantillas y arquitecturas para validar cualquier elemento en el vehículo, ya sea en un consultor de ingenieros o en un entorno de fabricación de alto volumen.

Para realizar simulación, muchos de los trabajos encontrados en la literatura hacen uso principalmente de herramientas MathWorks [62]. MathWorks es el desarrollador líder de software de computación matemática. MATLAB, el lenguaje de la informática técnica, es un entorno de programación para el desarrollo de algoritmos, análisis de datos, visualización y cálculo numérico. Simulink es un entorno gráfico para simulación y diseño basado en modelos para sistemas integrados y dinámicos multidominio. En particular, los modelos de Simulink se utilizan en el desarrollo de la mayoría de los controladores de vehículos anteriores a ADAS. Matlab ha ampliado sus capacidades para el desarrollo de ADAS con Autonomous Driving Toolbox, disponible a partir de la versión 2017b.

Esta herramientas proporciona algoritmos y herramientas para diseñar y probar ADAS y sistemas de conducción autónoma. Permite a los ingenieros automatizar el etiquetado de la verdad del terreno, generar datos de sensores sintéticos para escenarios de conducción, realizar una fusión de múltiples sensores y diseñar y simular sistemas de visión.

En la Tabla 9 se muestran algunos de los simuladores o plataformas de simulación utilizados para la validación y prueba de vehículos autónomos, siguiendo un enfoque basado en modelos.

\begin{tabular}{|c|c|c|c|c|c|c|}
\hline & & & & \multicolumn{3}{|c|}{ XIL } \\
\hline & License & $\begin{array}{c}\text { Open } \\
\text { Models }\end{array}$ & $\begin{array}{l}\text { ISO } 26262 \\
\text { Compliant }\end{array}$ & MIL & SIL & HIL \\
\hline PaTAVTT & GPL & $\mathrm{x}$ & $\mathrm{u}$ & & & $\mathrm{x}$ \\
\hline Simulink \&Matlab & Comercial & - & $\mathrm{x}$ & $\mathrm{x}$ & $\mathrm{x}$ & $\mathrm{x}$ \\
\hline dSpace $\mathrm{GmbH}$ & Comercial & - & $\mathrm{x}$ & $\mathrm{x}$ & $\mathrm{x}$ & $\mathrm{x}$ \\
\hline LabVIEW & Comercial & - & $\mathrm{x}$ & $\mathrm{x}$ & $\mathrm{x}$ & $\mathrm{x}$ \\
\hline CarSim & Comercial & $\mathrm{u}$ & $\mathrm{x}$ & $\mathrm{x}$ & $\mathrm{x}$ & $\mathrm{x}$ \\
\hline CAT Vehicle & GPL/ Open Source & $\mathrm{x}$ & $\mathrm{u}$ & $\mathrm{x}$ & $\mathrm{x}$ & $\mathrm{x}$ \\
\hline
\end{tabular}

Tabla 9. Resumen de las principales características de las plataformas de simuladores para VAs 


\subsection{DISEÑO DE UN SISTEMA DE TOMA DE DECISIÓN PARA CICAR}

El objetivo principal de la planificación es proporcionar al vehículo un camino seguro y sin colisiones hacia su destino, teniendo en cuenta la dinámica del vehículo, sus capacidades de maniobra en presencia de obstáculos, junto con las normas de tránsito y los límites de las carreteras [55]. La planificación es una rutina que consume memoria, así como una rutina de computación intensiva, que se ejecuta en paralelo con otras operaciones rutinarias del vehículo (por ejemplo, seguimiento de obstáculos, fusión de datos y módulos de control). Las entradas y salidas de una planificación de movimiento normalmente dependen de estos otros módulos. La planificación confiable, robusta y adaptable es esencial, especialmente en un escenario de tráfico urbano mixto. Estos algoritmos reciben entradas del marco del sensor y complementan estas entradas con datos de mapas de ruta digitales para proporcionar un espacio de trabajo completo en el que se lleva a cabo la planificación

La arquitectura propuesta, como sistema de decisión está basada en un sistema tradicional formado por (1) planificador global PPS, (2) planificador local PPL, (3) un sistema análisis de diagnosis y análisis de fallas, (4) sistema de gestión de energía, (5) y un sistema de comunicación, ver Figura 36.

La arquitectura propuesta es un sistema modular y abierto capaz de integrar fácilmente la amplia variedad de sensores y actuadores. En este trabajo de tesis se ha abordado el diseño e implementación del planificador global y planificador local [69].

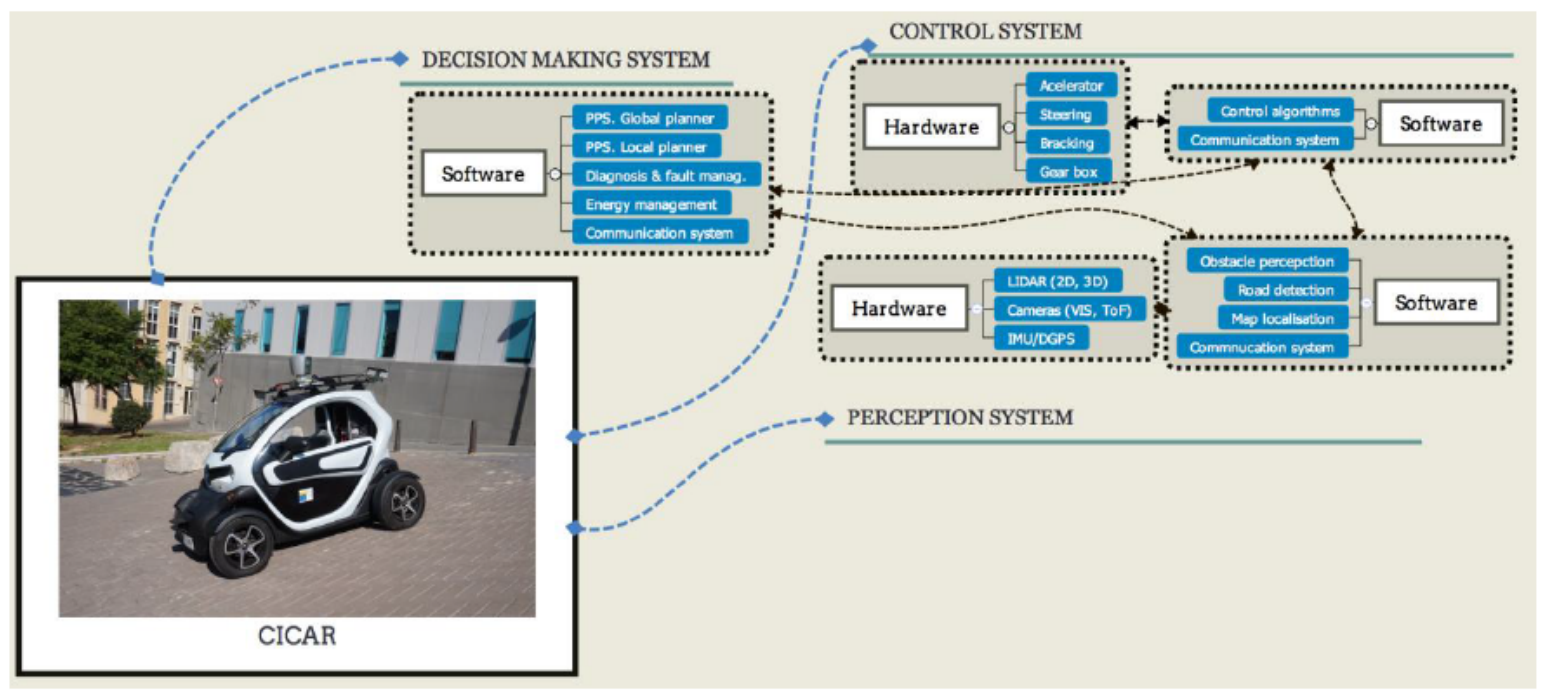

Figura 36. Distribución de los componentes de arquitectura de alto nivel del CICar.

A continuación se muestra más detalle estos subsistemas. 


\subsubsection{PLANIFICADOR GLOBAL}

Los planificadores globales son un elemento clave en los sistemas de navegación autónomos. El desarrollo de los sistemas de transporte inteligentes y vehículos autónomos han aumentado la demanda de planificadores de rutas que permiten la generación de rutas dinámicas, capaces de adaptarse a los diferentes aspectos de la carretera, como tráfico, obras viales, caminos que han sido cortados o eventos inesperados. Los requisitos iniciales para los planificadores globales de CICar se enumeran a continuación:

(1) Debe ser capaz de crear rutas globales utilizando fuentes de mapas digitales

(2) Debe permitir una introducción fácil de las restricciones de tráfico o eventos no planeados.

(3) Debe ejecutarse de manera ágil y producir resultados en un tiempo aceptable.

Para la fuente de mapa utilizada para la planificación global de rutas, se seleccionó Google maps, las razones para este fueron:

(1) Tiene una interfaz de programación de aplicaciones (API) de fácil acceso y es multiplataforma.

(2) Los mapas se pueden descargar con diferentes capas de información.

(3) Permite georreferenciar los puntos del mapa.

(4) Se actualiza constantemente y está disponible de forma gratuita.

El primer algoritmo evaluado para la generación global de rutas fue el algoritmo A* $[70][71]$.

La siguiente desventaja con el uso del algoritmo $A^{*}$ era que no es capaz de generar rutas en un tiempo razonable usando mapas binarios con resolución elevadas (512 x 512 o 800 x 600).

Para aumentar el rendimiento, el tamaño de los mapas binarios se redujo en un factor constante $(2,3$ o 4). La reducción de tamaño del mapa binario aceleró el tiempo de cálculo, pero debido a los efectos de pixelización, ocurrieron los siguientes problemas: (1) la destrucción de caminos estrechos, (2) el deterioro de las carreteras principales, y, como resultado, (3) las rutas generadas no fueron suaves.

Como solución a los problemas indicados, se desarrolló un nuevo algoritmo de búsqueda para la planificación de rutas globales, llamado SCP (Búsqueda de puntos cruzados para obtener rutas globales). El algoritmo SCP se basa en un mapa binario de píxeles $\mathrm{n} \times \mathrm{m}$ donde las rutas navegables reciben una valor de "1" y las áreas no navegables un valor de "0", punto inicial $\mathrm{p}_{0}$, punto de meta $\mathrm{p}_{\mathrm{g}}$, y un el ángulo inicial $\theta_{0}$. 
Las etapas realizadas por el algoritmo SCP para obtener la ruta global son:

1. Se elige una dirección de búsqueda ('N'-North,' S'-South, 'E'-East,' W'-West) según la posición del ángulo entre las posiciones $\mathrm{p}_{\mathrm{g}} \mathrm{y}_{0}$. La ecuación (40) muestra cómo son las direcciones de búsqueda obtenido por el algoritmo en función del ángulo $\Theta_{0 \mathrm{~g}}$

$$
\text { directions }=f\left(\theta_{0 g}\right)=\left\{\begin{array}{cc}
N, & -45 \leq \theta_{0 g}<-135 \\
W, & -135 \leq \theta_{0 g}<135 \\
S, & 135 \leq \theta_{0 g}<45 \\
E, & 45 \leq \theta_{0 g}<-45
\end{array}\right.
$$

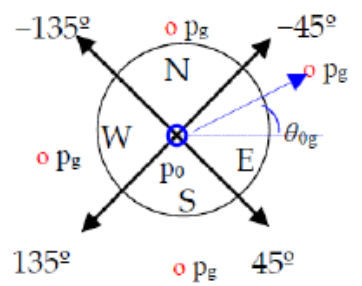

2. Se elige un camino directo sin obstáculos desde $\mathrm{p}_{0}$ hasta el objetivo $\mathrm{p}_{\mathrm{g}}$. Una ruta se considera directa si todos los puntos que forman la línea que une $\mathrm{p}_{0} \mathrm{y} \mathrm{p}_{\mathrm{g}}$ tienen un valor igual a ' 1 '.

$2.1 \mathrm{Si}$ el camino no es libre:

a. El algoritmo SCP busca el primer par de puntos cruzados $[\mathrm{CPi} ; \mathrm{CP} i+1]$ según la dirección de búsqueda establecida como se muestra en la ecuación (40). Para lograr esto, el algoritmo va a través del mapa, comenzando desde p0 según un ángulo $\boldsymbol{\oplus}$, hasta que encuentre un valor igual a ' 0 '. El punto encontrado se etiqueta como el primer punto cruzado CPi. A partir de $\mathrm{CPi}$, y de acuerdo con el ángulo de búsqueda $\bigoplus$, el algoritmo busca el siguiente punto con un valor de ' 0 '. Este nuevo punto se etiqueta como el segundo punto de cruce $\mathrm{CPi}+1$. Los ángulos $\boldsymbol{\oplus}, \boldsymbol{\oplus} \mathbf{2}$ se calculan según la dirección de búsqueda y la Tabla 10.

\begin{tabular}{ccc}
\hline Directions & Angle $\boldsymbol{\theta}_{1}$ & Angle $\boldsymbol{\theta}_{\mathbf{2}}$ \\
\hline $\mathrm{N}$ & $-180+\theta_{0}$ & $-\theta_{0}$ \\
$\mathrm{~S}$ & $\theta_{0}$ & $180-\theta_{0}$ \\
$\mathrm{E}$ & $\theta_{0}$ & $-\theta_{0}$ \\
$\mathrm{~W}$ & $-180+\theta_{0}$ & $180-\theta_{0}$ \\
\hline Tabla 10. Cálculo de ángulos $\boldsymbol{\theta}_{1}, \boldsymbol{\theta}_{2}$, dependiendo de la dirección
\end{tabular}

Durante la búsqueda de puntos cruzados $\left[\mathrm{CP}_{\mathrm{i}} ; \mathrm{CP}_{\mathrm{i}+1}\right]$, los puntos candidatos $\mathrm{p}_{\mathrm{i}}\left(\mathrm{x}_{\mathrm{i}}, \mathrm{y}_{\mathrm{i}}\right)$ son calculado al alternar los valores de $\boldsymbol{\theta}=\boldsymbol{\theta}_{1}$ y $\boldsymbol{\theta}=\boldsymbol{\theta}_{2}$, en base a la ecuación (41):

$$
\begin{aligned}
& x_{i}=x_{i-1}+d \cos (\theta) \\
& y_{i}=y_{i-1}+d \sin (\theta)
\end{aligned}
$$

siendo $\left(\mathrm{x}_{\mathrm{i}-1}, \mathrm{y}_{\mathrm{i}-1}\right)$ el valor previo calculado, $\mathrm{d}$ una constante que permite la búsqueda de $\mathrm{CP}_{\mathrm{i}}$ para avanzar más rápido.

b. La distancia euclidiana entre dos puntos de cruce consecutivos $\mathrm{CPi}$ y $\mathrm{CPi}+1$ es calculado.

b1. Si la distancia es 0 , la dirección de búsqueda se cambia al evaluar la posición de pg con respecto al último $\mathrm{CPi}+1$ que se calculó, p0 se establece en $\mathrm{p} 0=\mathrm{CPi}+1$, y el algoritmo va al paso 2 .

b2. Si la distancia no es igual a 0 , p0 se establece en $\mathrm{p} 0=\mathrm{CPi}+1$, y el algoritmo va al paso 2 . 


\section{$2.2 \mathrm{Si}$ hay una ruta directa:}

el algoritmo finaliza, y la ruta se compone de los puntos $[\mathrm{p} 0, \mathrm{pm} 1, \ldots, \mathrm{pmn} 1 ; \mathrm{pg}]$, con pm1,..., siendo pmn1 los puntos medios obtenidos de los pares de puntos del vector de punto de cruce $[\mathrm{p} 0, \mathrm{CP} 1, \mathrm{CP} 2, \ldots, \mathrm{CPn} ; \mathrm{pg}]$.

La Figura 37 muestra un mapa binario en forma de un cuadrado que demostrará el algoritmo SCP en la operación. La Figura 37a muestra cómo el algoritmo SCP genera los puntos de cruce en la dirección ' $\mathrm{N}$ ', dada la información $\left(\mathrm{p}_{0}, \mathrm{p}_{\mathrm{g}}, \boldsymbol{\oplus}_{0}\right)$. En el ejemplo con $\boldsymbol{\oplus}_{0}=60^{\circ}$ y la Tabla 10 , los ángulos $\boldsymbol{\oplus}_{1} \mathrm{y} \boldsymbol{\oplus}_{2}$ son $120^{\circ}$ y $60^{\circ}$ respectivamente.

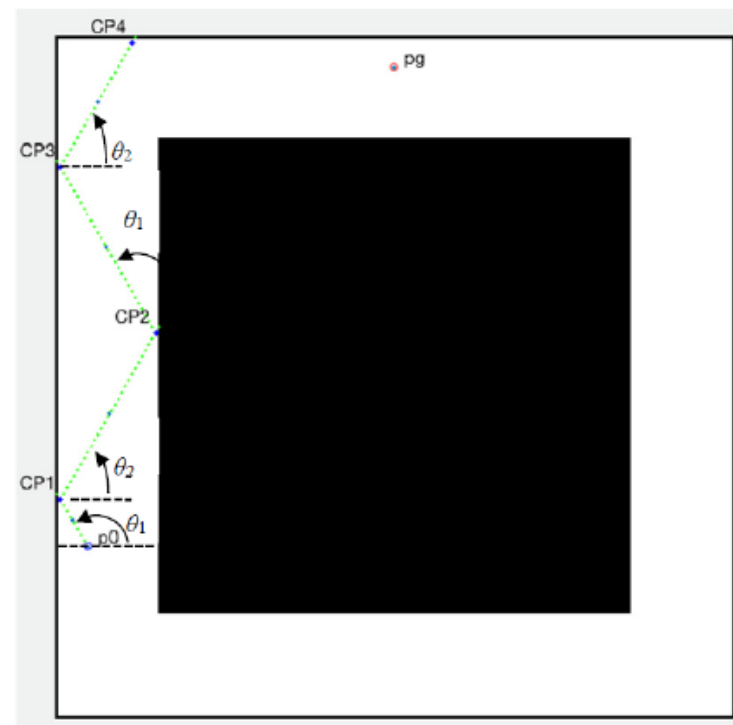

(a)

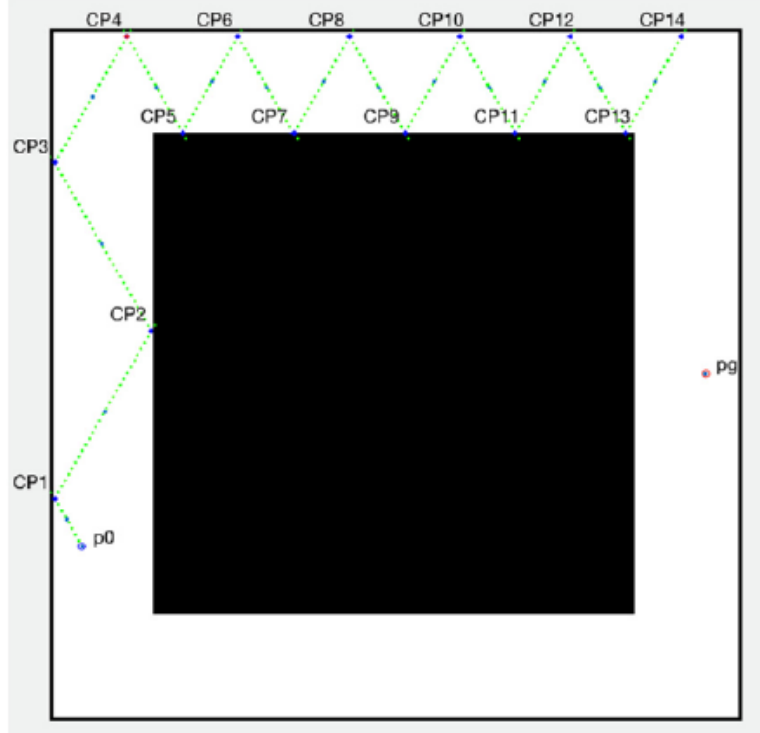

(c)

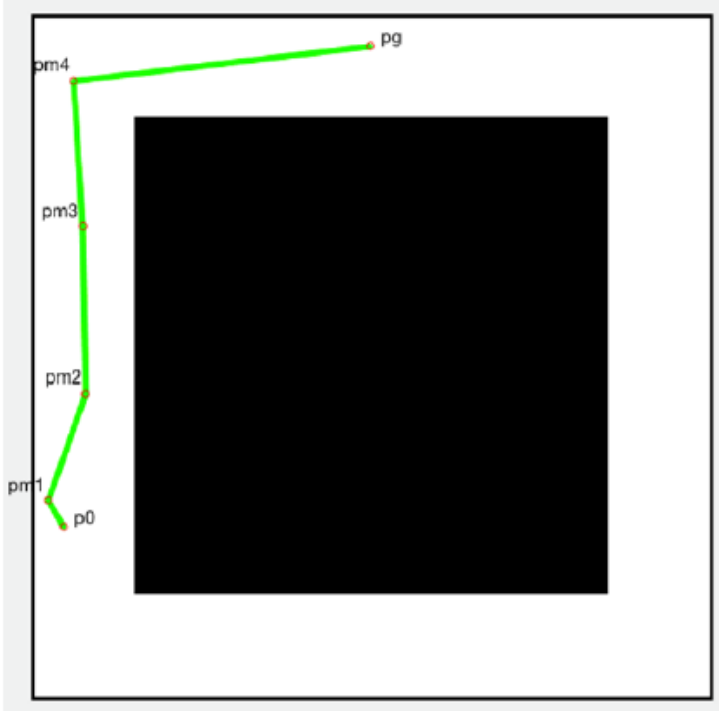

(b)

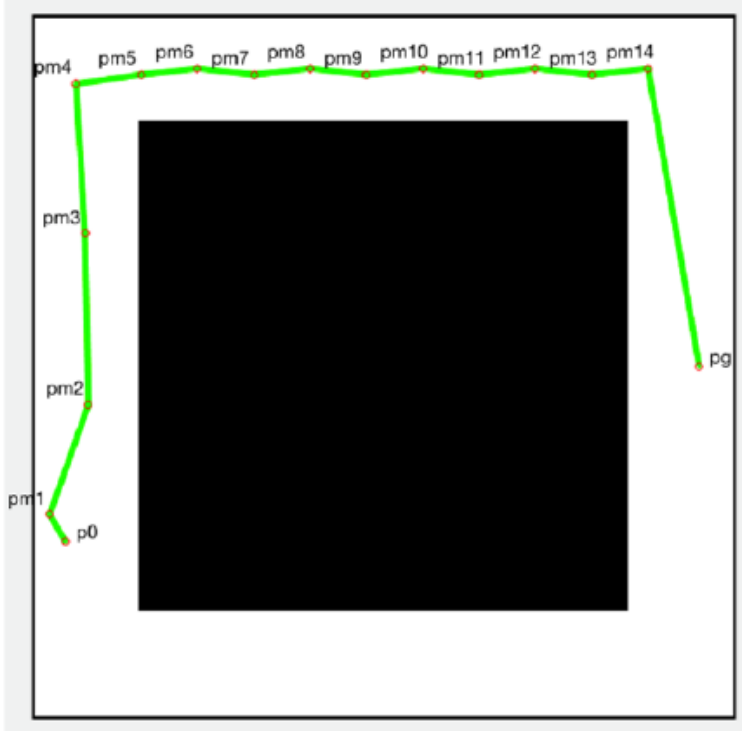

(d)

Figura 37. Ejemplo de la búsqueda de puntos cruzados $(\mathrm{SCP})$ que trabaja entre $\mathrm{p}_{0}$ y $\mathrm{p}_{\mathrm{g}}$ con ángulo inicial $\theta=60^{\circ}$

(a) Escenario sin cambio de dirección; (b) Trayectoria obtenida en el escenario sin cambio de dirección; (c) Escenario con cambio de dirección; (d) Trayectoria obtenida en el escenario con cambio de dirección. 
Como se muestra en la Figura $37 \mathrm{a}$, los puntos de cruce $\mathrm{CP}_{\mathrm{i}}$ se generan en zigzag en la dirección ' $\mathrm{N}$ ' hasta que se encuentra la ruta directa al destino. La Figura 37b muestra la ruta generada entre los puntos medios de los segmentos del conjunto $\left[\mathrm{p}_{0} \mathrm{CP}_{1} ; \mathrm{CP}_{1} \mathrm{CP}_{2} ; \mathrm{CP}_{2} \mathrm{CP}_{3} ; \mathrm{CP}_{3} \mathrm{CP}_{4}\right.$; $\mathrm{CP}_{4} \mathrm{P}_{\mathrm{g}}$. Se muestra en la Figura 37c, un complejo escenario con una $\mathrm{p}_{\mathrm{g}}$ diferente. En este caso, cuando el algoritmo $\mathrm{SCP}$ alcanza $\mathrm{CP}_{5}$, el final de la ruta concluye cuando ya no sigue la dirección 'N'. En este punto, la distancia euclidiana entre $\mathrm{CP}_{4}$ y $\mathrm{CP}_{5}$ es 0 , y el algoritmo cambia la dirección de búsqueda de ' $\mathrm{N}$ ' a 'E', con $\boldsymbol{\oplus}_{1}=60^{\circ}$ y $\boldsymbol{\oplus}_{2}=-60^{\circ}$. Con el cambio de dirección, es posible calcular un nuevo $\mathrm{CP}_{5}$. La Figura 54d muestra la ruta generada entre los puntos medios de los segmentos del conjunto $\left[\mathrm{p}_{0} \mathrm{CP}_{1}, \ldots, \mathrm{CP}_{14} \mathrm{p}_{\mathrm{g}}\right]$.

El algoritmo SCP puede aumentar el número de rutas calculadas con un mayor número inicial ángulos iniciales. La Figura 38a muestra un mapa real de la Universidad Politécnica de Cartagena donde se ha seleccionado un punto inicial $\mathrm{p}_{0} \mathrm{y}$ un punto final $\mathrm{p}_{\mathrm{g}}$ (cruces azules y rojas). El algoritmo SCP se ha ejecutado con ángulos iniciales $\emptyset_{0}$ de 20 a 80 grados, con incrementos de 2 grados Después de su ejecución, el SCP ha calculado tres posibles trayectorias. La Figura 38b muestra los puntos cruzados encontrados, y la Figura 38c las trayectorias creadas a partir del conjunto de puntos medios de las tres trayectorias calculadas. La Figura 38d muestra la ruta óptima calculada como la más corta entre los puntos $\mathrm{p}_{0} \mathrm{y} \mathrm{p}_{\mathrm{g}}$.

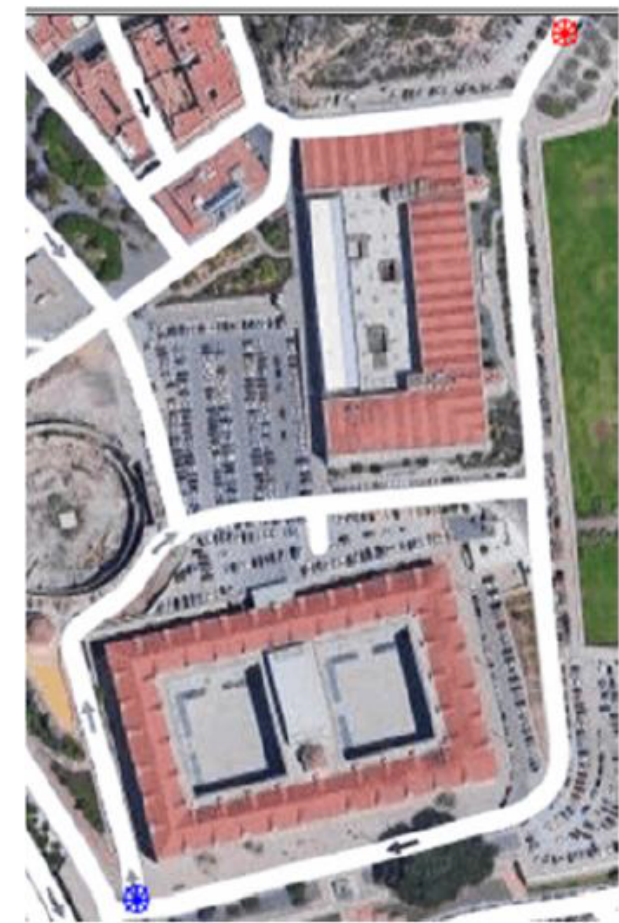

(a)

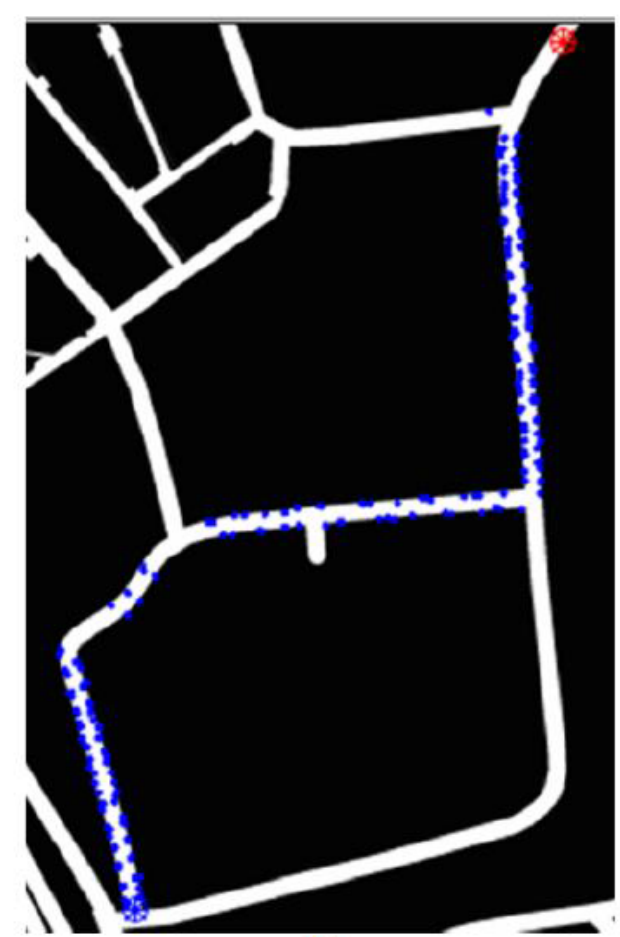

(b) 


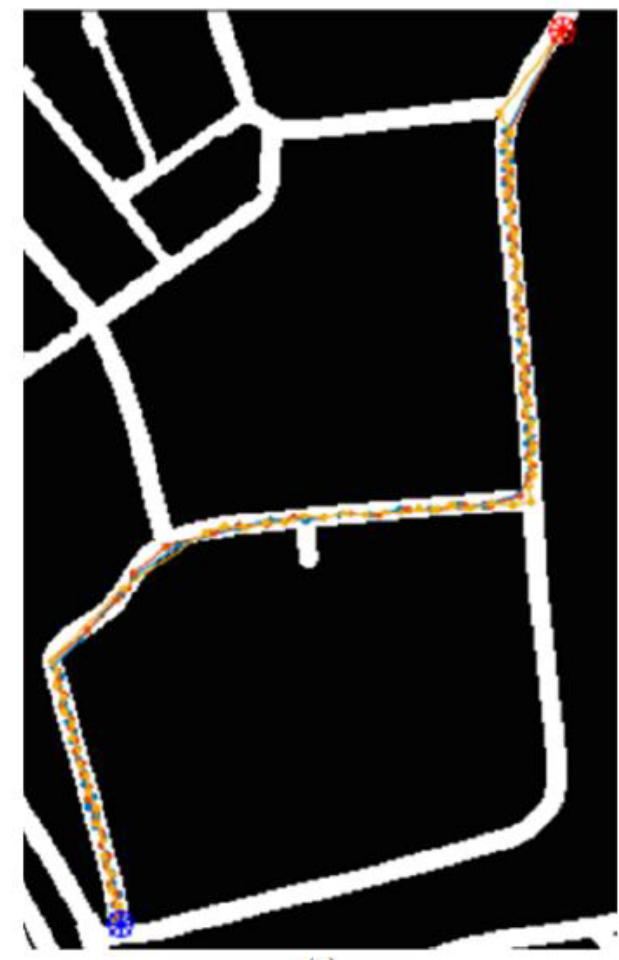

(c)

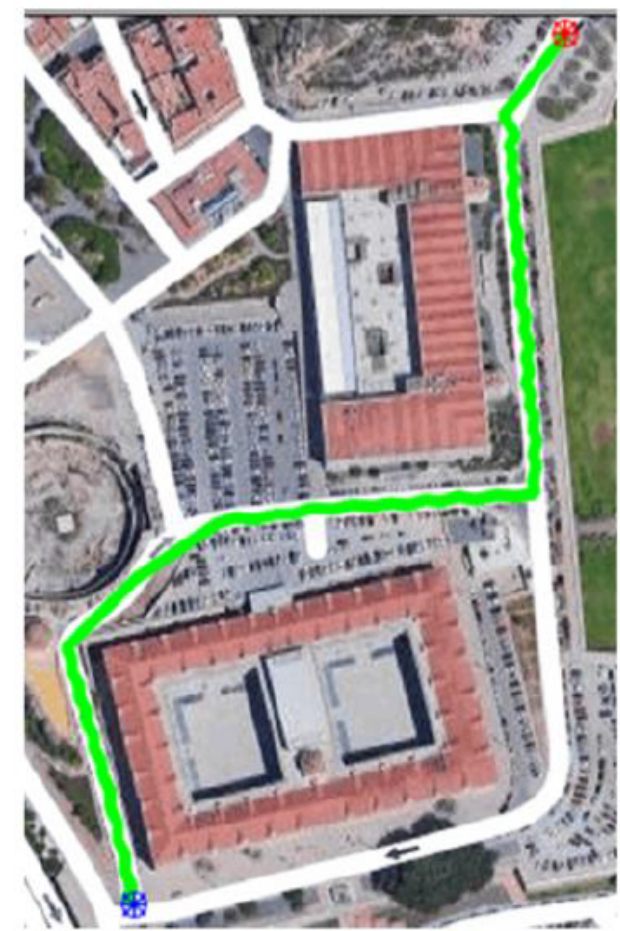

(d)

Figura 38. SCP aplicado en un mapa real. (a) Selección del punto inicial en el mapa real; (b) puntos encontrados en un mapa binario; (c) posibles trayectorias en un mapa binario; (d) trayectoria óptima en el mapa real

La ruta óptima está dividida en waypoints $\left(\mathrm{WP}_{0}, \ldots, \mathrm{WP}_{\mathrm{i}}, \ldots, \mathrm{WP}_{\mathrm{n}}\right)$. Los waypoints serán utilizados por el CICar para establecer misiones cortas entre ellos utilizando el módulo del planificador local. El CICar la ubicación es proporcionado por una unidad DGPS a bordo del vehículo. 


\subsubsection{PLANIFICADOR LOCAL}

El planificador local usa un par de waypoints $\left(W_{\mathrm{i}}, W_{\mathrm{i}+1}\right)$ suministrados por el planificador global para generar un conjunto de posibles trayectorias para llegar a $\mathrm{WP}_{\mathrm{i}+1}$ desde $\mathrm{WP}_{\mathrm{i}}$ (ver Figura 39).

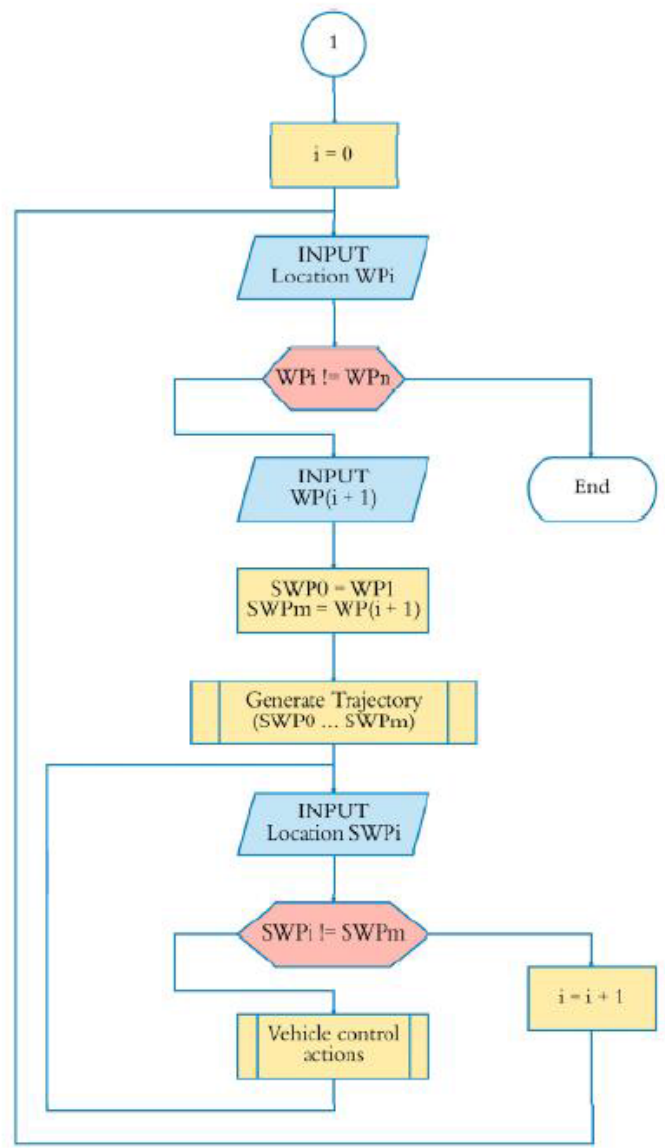

Figura 39. Diagrama flujo planificador local

Para eso, el sistema de percepción suministra un mapa local de hasta $100 \mathrm{~m}$ alrededor del vehículo (Figura 40a), que se completa con tres tipos de restricciones:

(a) las restricciones que el vehículo detecta en el camino mediante el sistema de percepción (vehículos, peatones, bicicletas, bordillos, etc.)

(b) las limitaciones inherentes a las características del vehículo (modelo ancho, alto, cinemático)

c) las limitaciones derivadas de las reglas de tráfico (unidireccional, bidireccional, señales de tráfico, etc.). Para disminuir el número de cálculos y para aumentar el rendimiento, el sistema de percepción proporciona un conjunto de puntos clave que son una representación del entorno del vehículo. El conjunto de puntos clave se obtiene después de aplicar un filtro de proceso compuesto por dos etapas:

1. Filtro de distancia. Todos los puntos XYZ más allá de una distancia máxima (dmax) serán rechazados.

2. Filtro de altura. Todos los puntos $\mathrm{Z}$ más altos que un valor máximo (Zmax) se descartarán. 
Como se muestra en la Figura 40b, hemos simplificado el mapa local XYZ inicial para obtener un perfil compacto de la representación XYZ de los objetos en la escena.
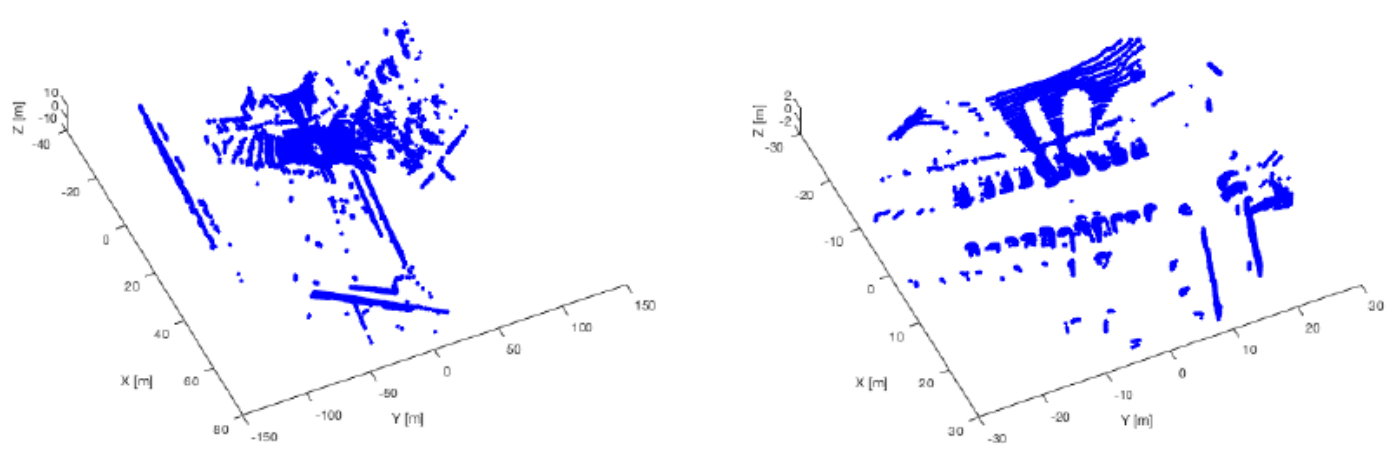

Figura 40. (a) Mapa local; (b) Mapa local filtrado

Después de esto, las esquinas de los objetos en la escena se calculan usando el algoritmo Harris [72]. El algoritmo Harris se aplica sobre un ROI (región de interés) en la dirección de avance del vehículo.

La Figura 41 muestra los resultados de la detección de esquinas en la ROI de dos escenarios extraídos de la Figura 41b. Este grupo de puntos calculados por el algoritmo Harris se denominan puntos clave $\left(\mathrm{KP}_{\mathrm{i}}\right)$ y se usarán en la próxima etapa del PPS para crear un conjunto de trayectorias

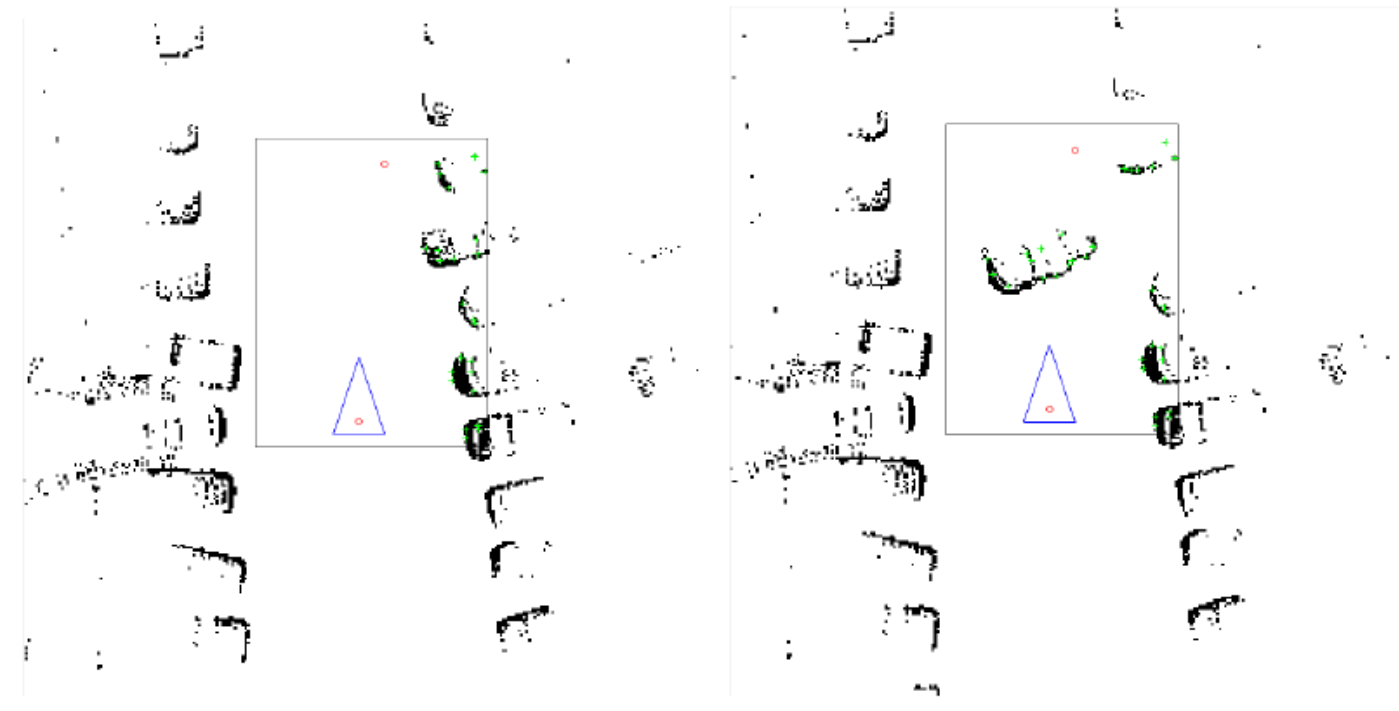

Figura 41. Waypoints $\left(\mathrm{WP}_{\mathrm{i}}, \mathrm{WP}_{\mathrm{i}+1}\right)$, ROI y puntos clave en dos escenarios

En la Figura 41, las cruces verdes representan el conjunto de puntos clave (KP $)_{\mathrm{s}}$, un triángulo con una base de 2 metros representa el vehículo, un rectángulo muestra el ROI donde se calcula el detector Harris, y dos círculos rojos representan las ubicaciones de origen y objetivo $\left(\mathrm{WP}_{\mathrm{i}}, \mathrm{WP}_{\mathrm{i}+1}\right)$.

El módulo generador de trayectoria recibe dos puntos de referencia $\left(\mathrm{WP}_{\mathrm{i}}, \mathrm{WP}_{\mathrm{i}+1}\right)$ y un conjunto de puntos clave $\left\{\mathrm{KP}_{0}, \ldots, \mathrm{KP}_{\mathrm{k}}\right\}$ representante del entorno alrededor del vehículo para calcular un conjunto de trayectorias entre un par de puntos de referencia. El módulo usa las curvas de Bezier para generar posibles trayectorias entre los waypoints, usando los puntos clave como puntos de control. 
Una curva de Bezier de grado n puede generalizarse mediante la ecuación siguiente [73]:

$$
B(t)=\sum_{i=0}^{n}\left(\begin{array}{l}
n \\
i
\end{array}\right) P_{i}(1-t)^{n-i} t^{i}, t \in[0,1]
$$

Por ejemplo, la curva de segundo grado $(n=2)$ es:

$$
B(t)=(1-t)^{2} P_{0}+2 \mathrm{t}(1-\mathrm{t}) P_{1}+\mathrm{t}^{2} P_{2}
$$

Siendo P0, P1 y P2 los puntos de control, y t un valor real entre 0 y 1 . Un valor más alto de t permite obtener un mayor número de puntos para la generación $\mathrm{B}(t)$ de la curva Bézier. El módulo generador de trayectoria implementa un conjunto de curvas de Bézier de segundo grado desplazadas a lo largo del eje X. El algoritmo de desarrollo se muestra en el algoritmo 1, donde nCurves indica el número de candidatos de trayectoria que se han calculado. Sin embargo, solo un conjunto superar las restricciones de distancia de seguridad y se adoptará como la trayectoria (Ti[]). Para cada uno de ellos, se define un nuevo punto de control P1, una mitad a la izquierda del punto medio entre P0 y P2, y la otra mitad a la derecha. Se define un incremento de desplazamiento del punto de control igual a 3.0. fB es una función que genera una curva de Bézier de segundo grado entre P0 y P2. La función fP1 busca el punto Pi con la distancia euclidiana más baja para establecer los puntos Bézier de B(t)

Después de obtener un conjunto de trayectorias $\mathrm{Ti}=\left\{\mathrm{B}(t)_{0}, \ldots, \mathrm{B}(t)_{\mathrm{m}}\right\}$, se puede establecer la trayectoria óptima bajo diferentes criterios dependiendo de las condiciones en cada momento durante la conducción autónoma.

En esta tesis, se han implementado tres criterios para seleccionar la trayectoria óptima: (a) la mínima distancia recorrida, (b) el ángulo máximo recorrido, y (c) la trayectoria media.

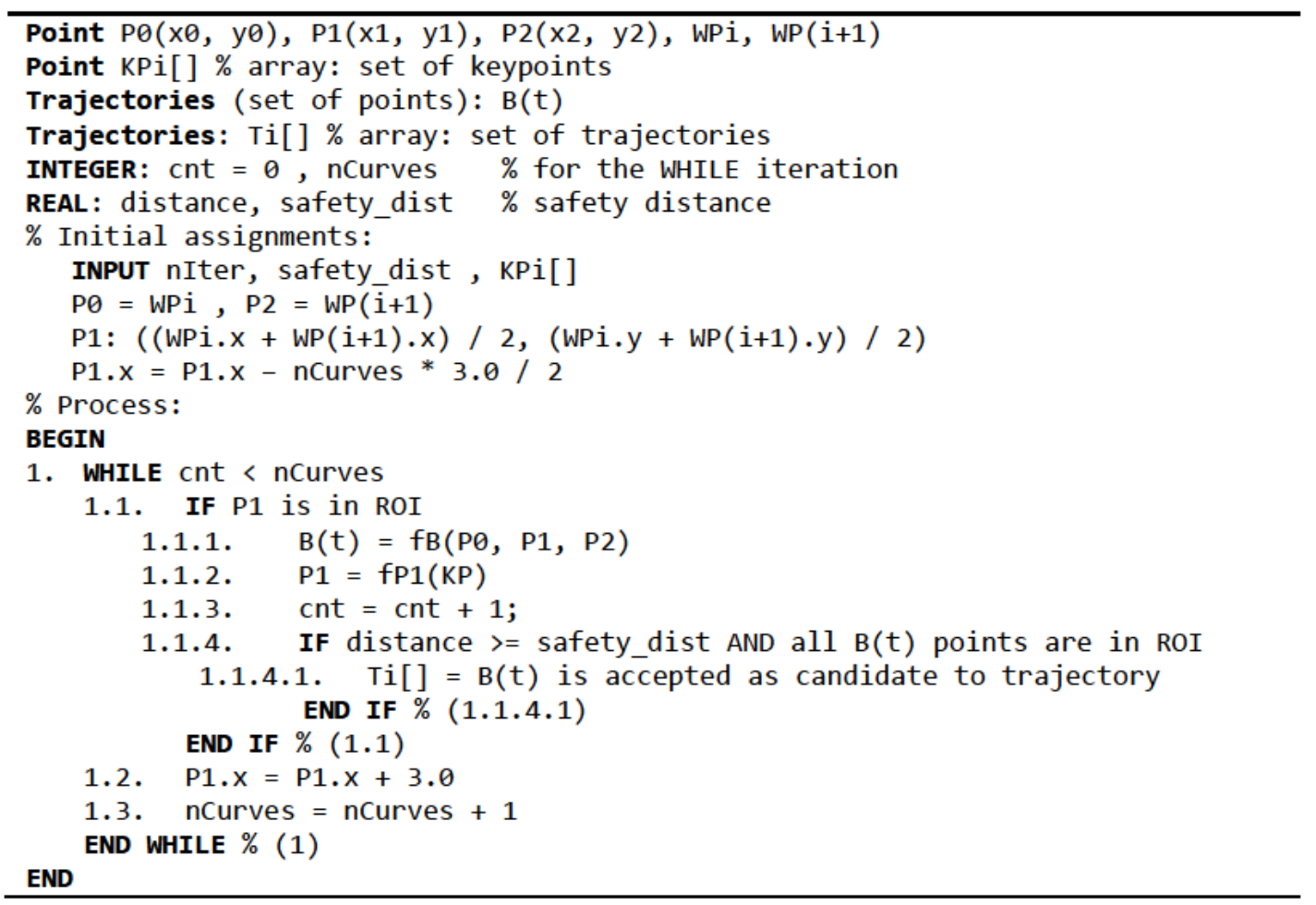

Figura 42. Algoritmo generador de trayectorias 
La trayectoria óptima devuelve un nuevo grupo de subwaypoints $\left(\mathrm{SWP}_{0}, \ldots, \mathrm{SWP}_{\mathrm{i}}, \ldots\right.$, $\left.\mathrm{SWP}_{\mathrm{n}}\right)$, que comprenda la ruta local entre $\left(\mathrm{WP}_{\mathrm{i}}, \mathrm{WP}_{\mathrm{i}+1}\right)$ bajo restricciones locales y medioambientales. Para mostrar cómo funciona el algoritmo del generador de trayectorias, se han seleccionado cuatro escenarios donde un vehículo conduce e interrumpe la trayectoria inicial (escenario 1, Figura 43a) entre $\mathrm{WP}_{\mathrm{i}}$ a $\mathrm{WP}_{\mathrm{i}+1}$ de los CICar. Los escenarios (1 a 4, Figura 43) presentan una complejidad creciente, donde la trayectoria el generador debe recalcular el conjunto de trayectorias dependiendo de la nueva ubicación de los puntos clave.

La Figura 43 muestra cómo el generador de trayectoria es capaz de resolver una situación de tráfico normal exitosamente. El módulo del generador de trayectoria se configuró con 30 iteraciones y una distancia de seguridad de $1 \mathrm{~m}$, y los criterios óptimos de la trayectoria se establecieron en la distancia más baja recorrida. En todos los escenarios propuestos en la Figura 43, el módulo generador de trayectoria generó 30 trayectorias, pero que cumplieran la distancia de seguridad solo se aprobaron: 16 en el escenario 1, 11 en el escenario 2, 1 en el escenario 3 y 2 en el escenario 4. En cada conjunto de trayectorias se seleccionó la trayectoria óptima según la distancia más baja recorrida.

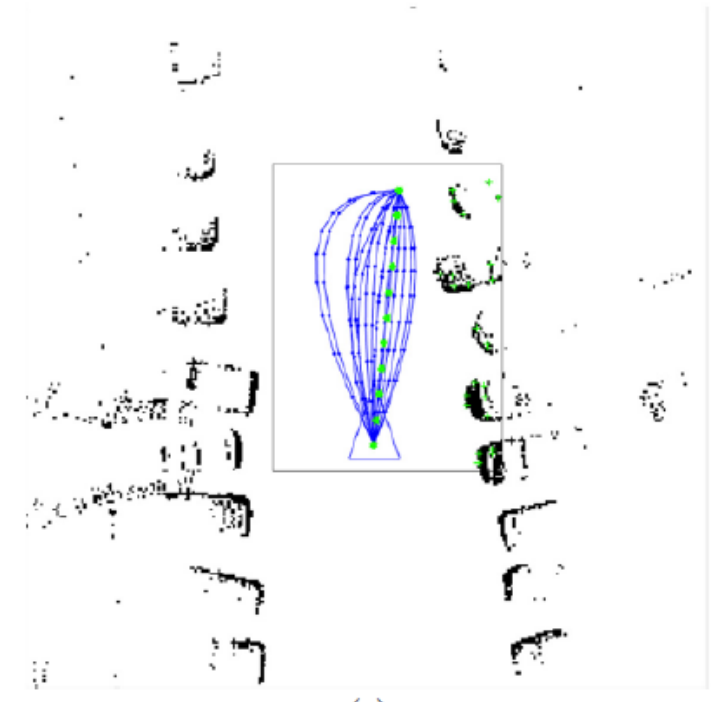

(a)

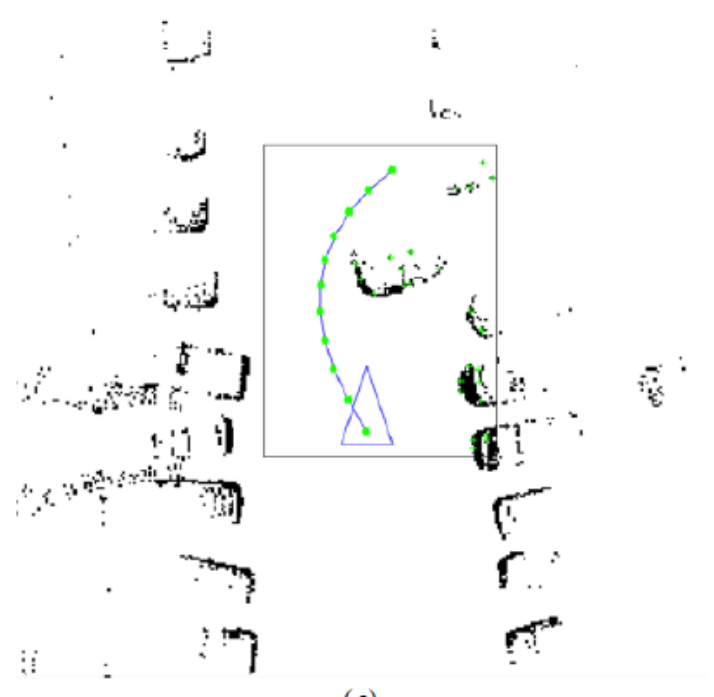

(c)

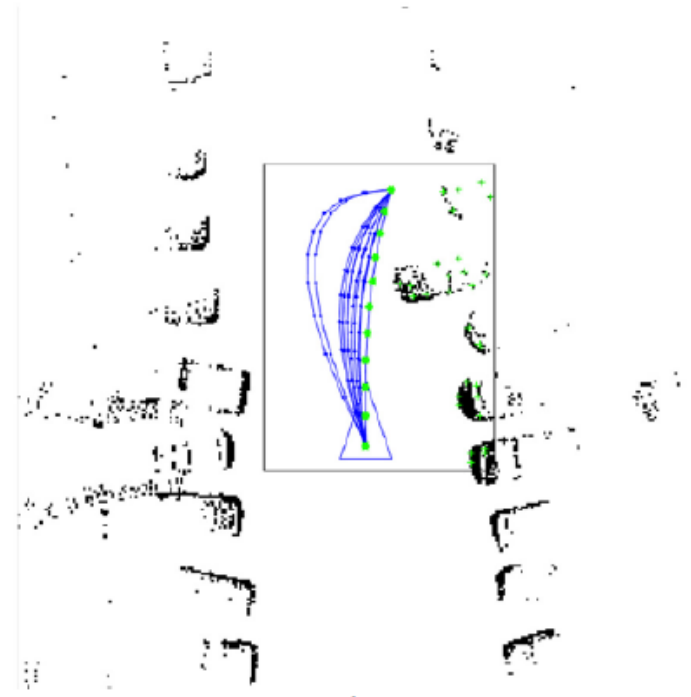

(b)

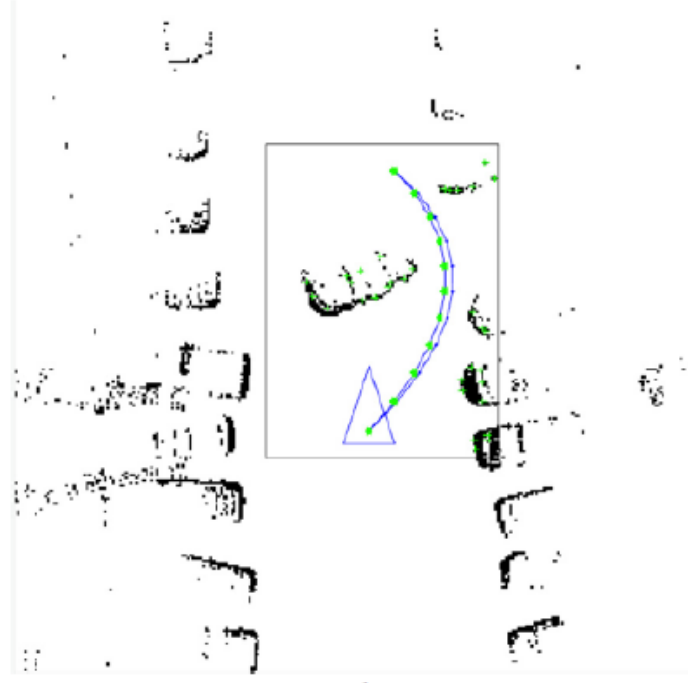

(d)

Figura 43. (a) escenario 1; (b) escenario 2; (c) escenario 3; (d) escenario 4 (nCurves $=30$, distancia de seguridad $=1 \mathrm{~m}$, criterios $=$ distancia mínima 
La Figura 44 muestra los comportamientos del generador de trayectoria cuando son elegidos otros criterios y parámetros del algoritmo. En la figura 44a, 44b, han sido seleccionados los criterios de ángulo máximo y de trayectoria del medio para el escenario 1, respectivamente. Es posible observar que el cambio en la selección, los criterios afectan la suavidad de la trayectoria. En la Figura 44c, 44d, el número de posibles trayectorias era establecido en 25. Con este cambio en los criterios, el generador de trayectoria no puede evitar el obstáculo y no puede suministrar solución en este escenario. Sin embargo, una disminución de la distancia de seguridad a $0,5 \mathrm{~m}$ permite generar cuatro trayectorias fiables (ver Figura 44d).

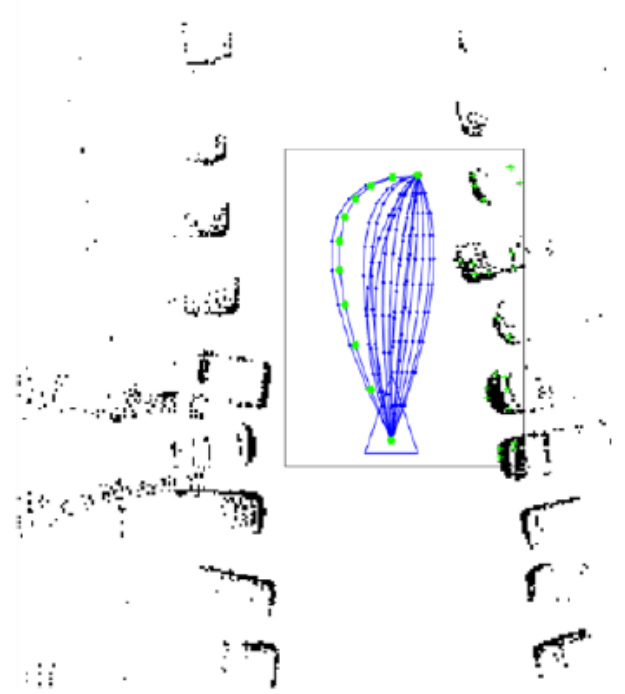

(a)

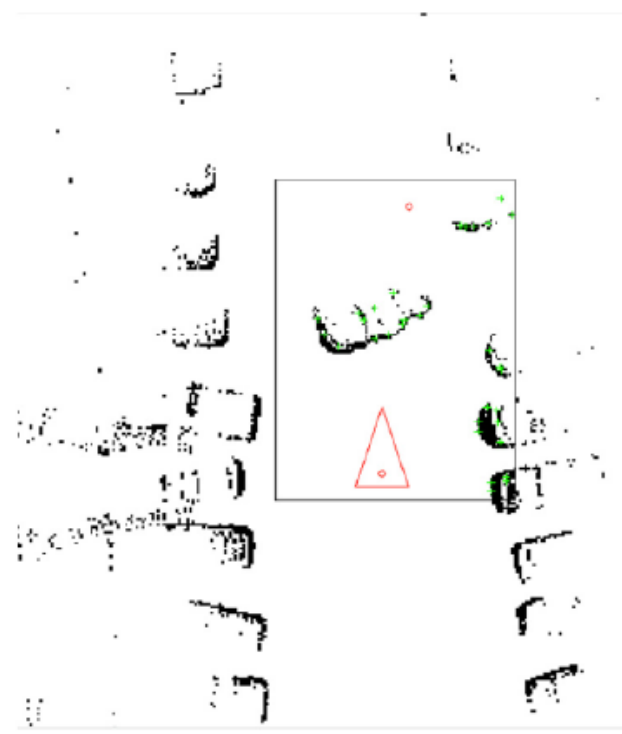

(c)

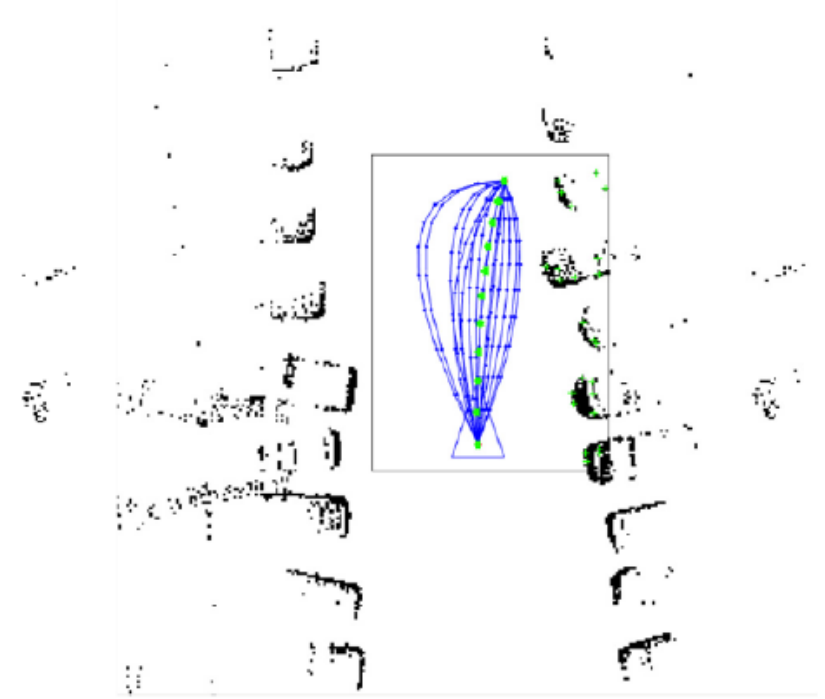

(b)

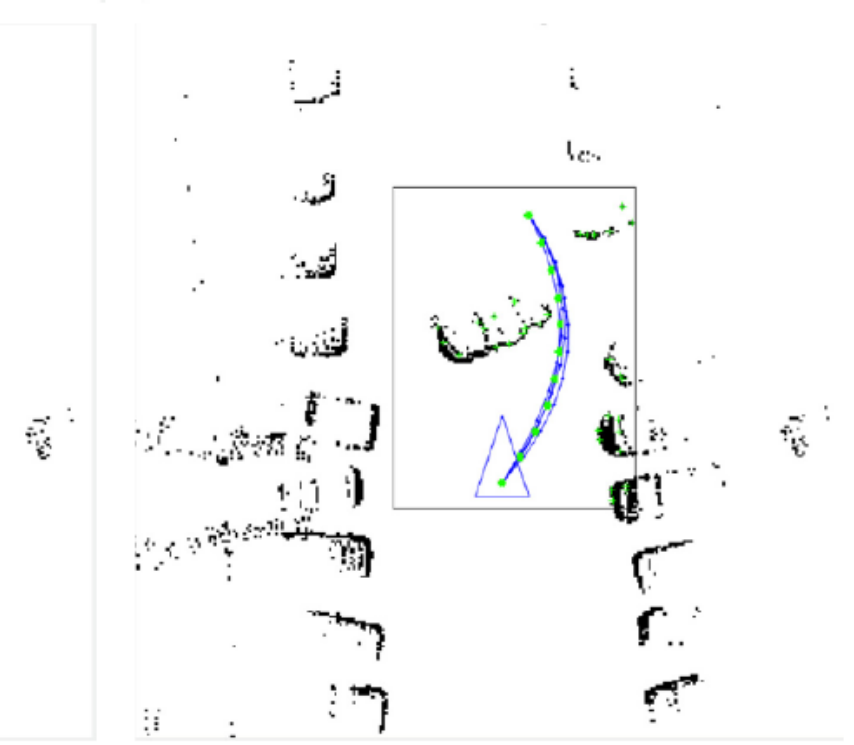

(d)

Figura 44

(a) escenario 1 (nCurves $=30$, distancia de seguridad $=1 \mathrm{~m}$, criterios = ángulo máximo);

(b) escenario 1 (nCurves $=30$, distancia de seguridad $=1 \mathrm{~m}$, criterios $=$ trayectoria media);

(c) escenario 4 (nCurves $=25$, distancia de seguridad $=1 \mathrm{~m}$, criterio $=$ distancia mínima);

(d) escenario 4 (nCurves $=25$, distancia de seguridad $=0.5 \mathrm{~m}$, criterios $=$ distancia mínima 
$6 \underline{\text { CAPITULO } 6}$

\section{ALGORITMO DE DETECCION DE PEATONES \\ BASADO EN NUBES DE PUNTOS}

\subsection{INTRODUCCION}

La detección de peatones se aborda equipando diversos sistemas de sensores en los vehículos e incluso fusionando los datos generados por algunos de ellos. Las cámaras y la visión estereoscópica, los sensores láser, las cámaras de tiempo de vuelo y los sensores LIDAR 3D son algunos ejemplos del tipo de sensores empleados para lograr este objetivo. A pesar de todos los desarrollos relevantes en la detección de peatones, esta tarea todavía presenta desafíos significativos. Uno de ellos consiste en lograr un rendimiento confiable en condiciones de iluminación muy variables, como ocurre en condiciones reales de conducción.

La detección de peatones se ha estudiado en los últimos años, donde la información visual se ha utilizado preferentemente para detectarlos. En [74] [75] se pueden encontrar algunas revisiones muy exhaustivas sobre el estado actual de la técnica. Las obras enumeradas en estos artículos están relacionadas principalmente con métodos de visión artificial para detectar peatones en imágenes, y examinan los algoritmos y conjuntos de datos más conocidos. Normalmente, los algoritmos de detección y conteo de peatones siguen un enfoque directo o indirecto. Los enfoques directos se basan en la segmentación peatonal y el seguimiento de cada individuo en la escena, que normalmente requiere algoritmos de visión por computadora [76]. El enfoque indirecto está basado en mapas, estimando el número de peatones en la escena por área.

En cuanto a los enfoques directos, se han aplicado diferentes características visuales para detectar peatones, como la información estereoscópica [77], el movimiento (por ejemplo, flujo óptico) [78] o la apariencia (por ejemplo, características locales como el histograma de degradados orientados [79]]). El uso de este tipo de histogramas representa uno de los desarrollos más importantes para mejorar los algoritmos que detectan peatones. Pero, aunque su uso ha demostrado un rendimiento satisfactorio en conjuntos de imágenes de alta calidad, los resultados en entornos de conducción reales no son tan satisfactorios [74]. La irrupción de sensores LIDAR en el mercado ha permitido utilizarlos como detectores de objetos y generalmente están compuestos por un conjunto de emisores láser que giran dentro de una carcasa. Devuelven mediciones para diferentes ángulos horizontales, verticales y dado que se componen de un número "discreto" de láseres, las áreas entre los puntos medidos no se detectan. Por lo tanto, generalmente no es posible reconstruir completamente la geometría del objeto detectado, el entorno se mide en diferentes momentos, lo que dificulta aún más el procesamiento de los datos. A pesar de las desventajas antes mencionadas, los sensores LIDAR se utilizan ampliamente debido a su precisión en varios tipos de aplicaciones, no solo navegación autónoma [80], sino también generación de modelos de túnel de metro [81], agricultura de precisión [82] e identificación de señalización [83], por mencionar solo algunos. 


\subsection{D LIDAR NUBE DE PUNTOS}

El LIDADR Velodyne HDL-64E envía la información (distancia al punto y valor de reflectividad) a través de paquetes UDP, configurados para transmitir en el puerto 2368. Cada paquete contiene una carga útil de datos de 1206 bytes, que comprende 12 bloques de 100 bytes de datos láser, más 6 bytes de calibración y otra información perteneciente al sensor. Cada bloque contiene datos de 32 láseres (3 bytes por láser), más información sobre el número de bloque láser (2 bytes) y el ángulo acimutal (2 bytes). El ángulo acimutal varía de 0 a 35.999 (décimas de grado) y las mediciones dadas por cada sensor láser, dos bytes codifican la distancia y el byte restante informa la intensidad en una escala de 0-255. Seis disparos de cada uno de los bloques de láser toman 139 us, más alrededor de 100 us para transmitir todo el paquete UDP de 1248 bytes. Los puntos de datos pueden transformarse en un sistema de coordenadas cartesianas aplicando las ecuaciones enumeradas a continuación, donde $\alpha$ es el ángulo acimutal y $\omega$ es el ángulo vertical.

$$
\begin{aligned}
& \mathrm{x}=\text { distancia } \cdot \cos (\omega) \cdot \sin (\alpha) \\
& \mathrm{y}=\text { distancia } \cdot \cos (\omega) \cdot \cos (\alpha) \\
& \mathrm{z}=\text { distancia } \cdot \sin (\omega)
\end{aligned}
$$

Las medidas de distancia son recolectadas por el LIDAR y organizadas en una nube de puntos que cubren los $360^{\circ}$ completos alrededor del vehículo. A su velocidad de rotación actual (10 $\mathrm{Hz}$ ), el LIDAR produce 1,3 millones de puntos por revolución, con una resolución angular de $0,1728^{\circ}$. Estos datos láser apenas se procesan previamente: solo los valores inferiores al mínimo (inferior a 500) se borran de la nube de puntos antes de pasarlos al algoritmo de detección de peatones, que se describe en la siguiente sección.

\subsection{ALGORITMO DE DETECCIÓN DE PEATONES}

A través del sistema de percepción del vehículo, con los sensores instalados (LIDAR3D+ToFs+LIDAR2D) se puede generar la nube de puntos, para el cálculo de este algoritmo solo se ha usado la información del LIDAR3D. El resto de sensores al trabajar en un rango muy corto, se utilizan para aspectos de seguridad, como por ejemplo el frenado de emergencia.

El algoritmo de detección de peatones descrito a continuación gira en torno al procesamiento de grupos de puntos contenidos dentro de un cubo de dimensiones 100x100x200 cm (base de datos de peatones etiquetados). El algoritmo comprende los siguientes cinco pasos:

1. Seleccionar los cubos que contienen una cierta cantidad de puntos, dentro de un umbral. Esto permite eliminar objetos con un número reducido de puntos que podrían producir falsos positivos. También reduce el tiempo de computación.

2. Generar proyecciones axonométricas XY, YZ y XZ de los puntos contenidos dentro del cubo.

3. Generar imágenes binarias de cada proyección y luego preprocesarlas.

4. Extraer las características de cada proyección axonométrica y datos brutos 3D LIDAR.

5. Procesar el vector de características para obtener si son o no peatón. 


\subsubsection{PASO 1. RECOPILACION DATOS PARA PROYECCIONES AXNOMETRICAS XY YZ XZ}

La Figura 61 muestra una captura de pantalla de todos los datos recopilados por Velodyne HDL-64 LIDAR en una revolución. Hemos resaltado dentro de un cubo rojo una persona, para que el lector pueda comprender mejor los siguientes pasos. La selección de cubos es el primer paso del algoritmo, y es muy simple. El umbral para aplicar el algoritmo descrito debajo al cubo está entre 150 y 4000 puntos, y estos números se han determinado empíricamente analizando tres mil marcos capturados por el LIDAR.

Figura 45. Muestra peatonal capturada con herramientas de software sobre un marco del 3D LIDAR

\subsubsection{PASO \\ 2: GENERACIÓN \\ PROYECCIONES \\ AXONOMETRICAS XY, YZ Y XZ}

Las proyecciones axonométricas XY, XZ y YZ se calculan a partir de una muestra que tiene suficientes puntos. La muestra se normaliza en función de sus valores máximos y mínimos locales de cada coordenada X, Y, Z según las ecuaciones enumeradas a continuación.

$$
\begin{aligned}
& X_{\text {norm }}^{(n)}=\frac{\mathrm{X}^{(n)}-\min \left(\mathrm{X}^{(n)}\right)}{\max \left(\mathrm{X}^{(n)}\right)-\min \left(\mathrm{X}^{(n)}\right)} \\
& Y_{\text {norm }}^{(n)}=\frac{\mathrm{Y}^{(n)}-\min \left(\mathrm{Y}^{(n)}\right)}{\max \left(\mathrm{Y}^{(n)}\right)-\min \left(\mathrm{Y}^{(n)}\right)} \\
& Z_{\text {norm }}^{(n)}=\frac{\mathrm{Z}^{(n)}-\min \left(\mathrm{Z}^{(n)}\right)}{\max \left(\mathrm{Z}^{(n)}\right)-\min \left(\mathrm{Z}^{(n)}\right)}
\end{aligned}
$$

Siendo n el número de la muestra y ns el número de puntos en la muestra

$$
\begin{gathered}
\mathrm{X}^{(n)}=\left\{x_{1}, \ldots, x_{n s}\right\}, \mathrm{Y}^{(n)}=\left\{y_{1}, \ldots, y_{n s}\right\}, \mathrm{Z}^{(n)} \\
=\left\{z_{1}, \ldots, z_{n s}\right\}
\end{gathered}
$$

son los conjuntos de coordenadas cartesianas de todos los puntos contenidos dentro de la muestra. Después de la normalización, los nuevos valores de los conjuntos $\left\{X_{\text {norm }}^{(n)}, Y_{\text {norm }}^{(n)}, Z_{\text {norm }}^{(n)}\right\}$ se colocan en el intervalo (0,1) La Figura 46 muestra las proyecciones axonométricas normalizadas de dos muestras de peatones. 


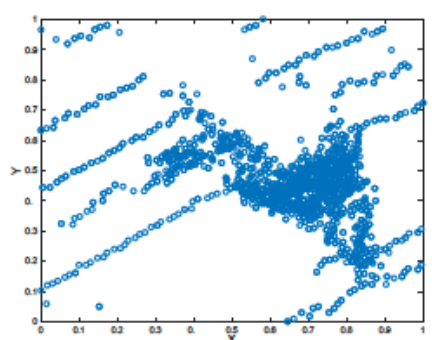

(a)

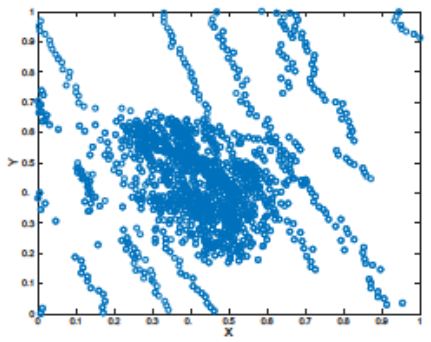

(d)

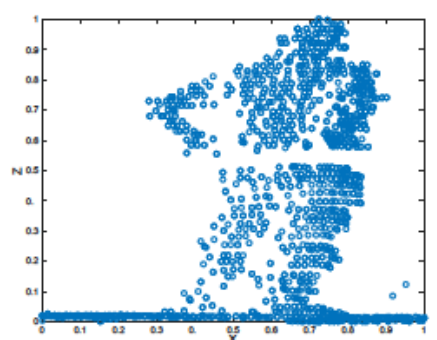

(b)

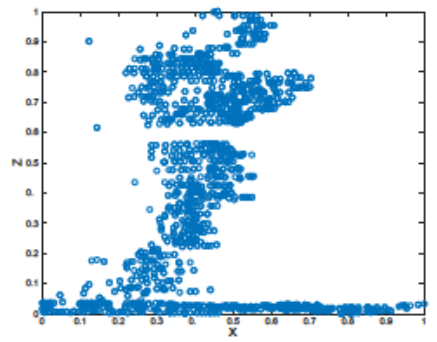

(e)

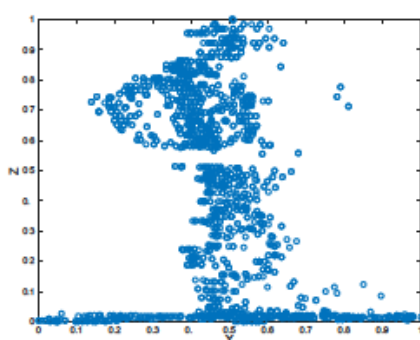

(c)

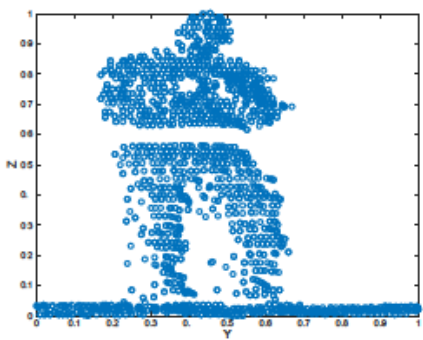

(f)

Figura 46. Proyecciones axonométricas XY, XZ y YZ normalizadas de dos muestras de peatones: (a), (b), (c) peatón uno; (d), (e), (f) peatón dos

\subsubsection{PASO 3: GENERACIÓN Y PREPROCESADO IMÁGENES BINARIAS PROYECCIONES AXONOMÉTRICAS}

Los valores normalizados obtenidos en el paso 2 se transforman entonces en una escala entera. Los nuevos valores mantienen las proporciones originales para evitar deformaciones de las proyecciones. Los nuevos puntos forman una imagen binaria: establecemos el fondo en negro (0) y las coordenadas de los puntos nuevos en blanco (1) en cada proyección. Por lo tanto, creamos una imagen binaria por proyección. Las Figuras 47 (a-f) muestran las imágenes binarias obtenidas de las muestras que se muestran en la Figura 46. Tenga en cuenta cómo la transformación no altera las proporciones de la muestra original.

Estas imágenes binarias se procesan previamente para eliminar partículas pequeñas, reducir el ruido, suavizar los objetos y obtener una figura compacta del objeto. Para este propósito, aplicamos dos operaciones morfológicas y una operación de filtro en el siguiente orden:

(1) una operación de apertura con radio de 6 píxeles para lograr un conjunto compacto de píxeles;

(2) un filtro para borrar partículas pequeñas con áreas de menos de 200 píxeles,

(3) un cierre con una relación de 3 píxeles para suavizar los bordes.

Las Figuras 47 (g-l) muestran resultados de la etapa de preproceso sobre las muestras de peatones. 

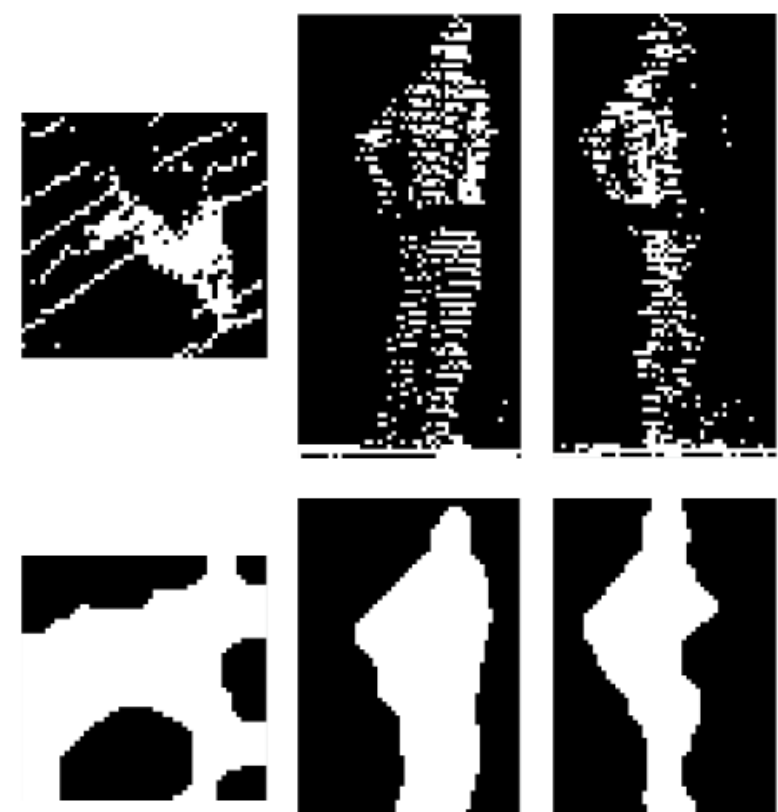

(g)

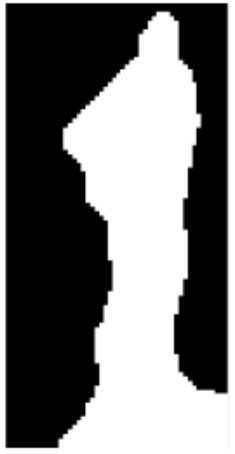

(h)

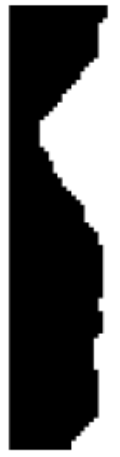

(i)

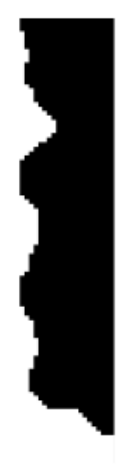

)
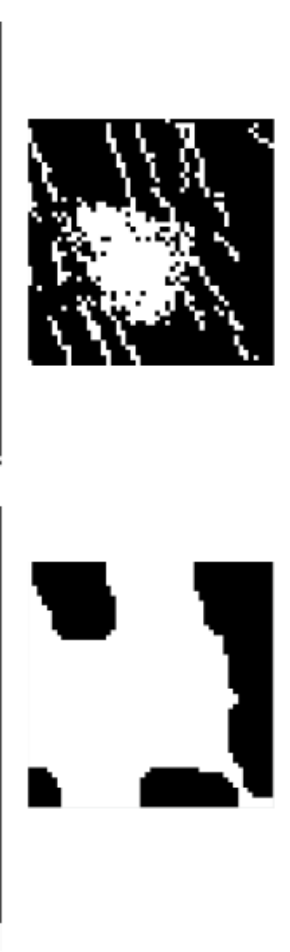

(j)
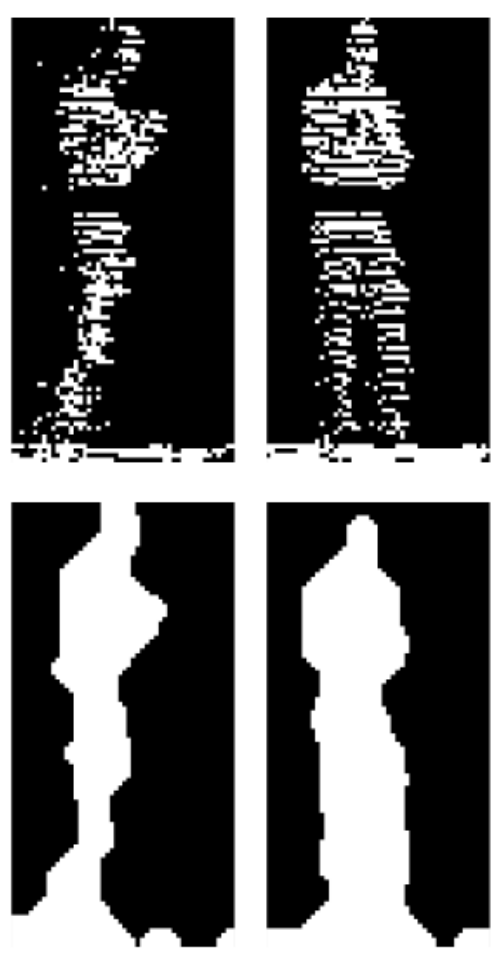

(k)

Figura 47. (a-f) imágenes binarias generadas a partir de las proyecciones XY, XZ y YZ de dos muestras de peatones; (g-l) resultados del pre-procesamiento de las imágenes binarias

\subsubsection{PASO 4: CÁLCULO DEL VECTOR DE CARACTERÍSTICAS}

En el aprendizaje automático o la visión por computadora, un vector de características es un vector n-dimensional de características numéricas que representan un objeto [84]. El tamaño del vector y las características que almacena depende de los objetos que desea buscar y de las condiciones en que se recopilaron los datos. Para aumentar o resaltar los datos brutos, las transformaciones en espacios como Fourier [85], Gabor [86] o Wavelet [87] se usan comúnmente. En otros casos, los datos brutos se reducen a formato binario (por ejemplo, imágenes en escala de grises a imágenes binarias) para encontrar las características geométricas del objeto [88], como área, perímetro, compacidad, excentricidad, etc. Otra técnica utilizada habitualmente es el cálculo de las estadísticas de primer y segundo orden [89] que también se han utilizado a menudo como características en los clasificadores y sistemas de aprendizaje automático [90].

Para separar a los peatones del resto de la información contenida en la nube de puntos, calculamos un vector de características compuesto por cincuenta características (f1, ..., f50) por proyección. Las características se pueden dividir en tres grupos. El primer grupo mide las características de forma. El segundo grupo mide los siete momentos invariantes de $\mathrm{Hu}$ [91]. El tercer grupo calcula la información estadística, específicamente, la distancia normalizada y la reflexividad informadas a partir de los datos LIDAR 3D. La Tabla 11 muestra un resumen de las cincuenta características contenidas en el vector, de acuerdo con el grupo al que pertenecen. 


\begin{tabular}{|c|c|c|}
\hline Shape features & Invariant moments & Statistical features \\
\hline $\begin{array}{c}f_{1} \ldots f_{3}: \text { Areas of } X Y, X Z, Y Z \\
\text { projections }\end{array}$ & $\begin{array}{c}f_{22} \ldots f_{24}: \text { Hu moment } 1 \text { over } X Y, X Z, Y Z \\
\text { projections }\end{array}$ & $\begin{array}{c}\mathrm{f}_{43}, \mathrm{f}_{44}: \text { Means of distances and } \\
\text { reflexivity }\end{array}$ \\
\hline $\begin{array}{c}f_{4} \ldots f_{8}: \text { Perimeters of } X Y, X Z, Y Z \\
\text { projections }\end{array}$ & $\begin{array}{c}\mathrm{f}_{25 \ldots} \ldots \mathrm{f}_{27}: \text { Hu moment } 2 \text { over } \mathrm{XY}, \mathrm{XZ}, \mathrm{YZ} \\
\text { projections }\end{array}$ & $\begin{array}{l}\mathrm{f}_{45}, \mathrm{f}_{46}: \text { Standard deviations of } \\
\text { distances and reflexivity }\end{array}$ \\
\hline $\begin{array}{c}\mathrm{f}_{7} \ldots \mathrm{f}_{9}: \text { Solidity of } X Y, X Z, Y Z \\
\text { projections }\end{array}$ & $\begin{array}{c}f_{28} \ldots f_{30}: \text { Hu moment } 3 \text { over } X Y, X Z, Y Z \\
\text { projections }\end{array}$ & $\begin{array}{c}\mathrm{f}_{47}, \mathrm{f}_{48}: \text { Kurtosis of distances and } \\
\text { reflexivity }\end{array}$ \\
\hline $\begin{array}{l}f_{10} \ldots f_{12}: \text { Equivalent diameters of } \\
X Y, X Z, Y Z \text { projections }\end{array}$ & $\begin{array}{c}f_{31} \ldots f_{33}: \text { Hu moment } 4 \text { over } X Y, X Z, Y Z \\
\text { projections }\end{array}$ & $\begin{array}{c}f_{49}, f_{50} \text { : Skewness of distances and } \\
\text { reflexivity }\end{array}$ \\
\hline $\begin{array}{c}f_{13 \ldots} \ldots f_{15}: \text { Eccentricity of } X Y, X Z \\
Y Z \text { projections }\end{array}$ & $\begin{array}{c}f_{34 . \ldots} f_{38}: \text { Hu moment } 5 \text { over } X Y, X Z, Y Z \\
\text { projections }\end{array}$ & \\
\hline $\begin{array}{c}f_{16} \ldots f_{18}: \text { Length major axis of } \\
X Y, X Z, Y Z \text { projections }\end{array}$ & $\begin{array}{c}f_{37} \ldots f_{39}: \text { Hu moment } 6 \text { over } X Y, X Z, Y Z \\
\text { projections }\end{array}$ & \\
\hline $\begin{array}{l}f_{19 . . .} f_{21} \text { : Length minor axis of } \\
X Y, X Z, Y Z \text { projections }\end{array}$ & $\begin{array}{c}\mathrm{f}_{40} \ldots \mathrm{f}_{42}: \text { Hu moment } 7 \text { over } \mathrm{XY}, \mathrm{XZ}, \mathrm{YZ} \\
\text { projections }\end{array}$ & \\
\hline
\end{tabular}

Tabla 11. Composición del vector de características

Los elementos de forma del vector de características son:

1. Área: número de píxeles del objeto.

2. Perímetro: especifica la distancia alrededor del límite de la región.

3. Solidez: proporción de los píxeles en el casco convexo también se encuentran en la región.

4. Diámetro equivalente: especifica el diámetro de un círculo con la misma área que el objeto (ver ecuación siguiente).

$$
E D=\sqrt{\frac{4 * \text { Area }}{\pi}}
$$

5. Excentricidad: relación de la distancia entre los focos de la elipse y su longitud del eje principal. El valor está entre 0 y 1.

6. Longitud del eje mayor / menor. Un escalar que especifica la longitud (en píxeles) del eje principal / secundario de la elipse que tiene los mismos segundos centrales normalizados que la región bajo consideración. 
Los momentos invariantes de $\mathrm{Hu}$ con respecto a la traducción, la escala y la rotación se calculan como se muestra en las ecuaciones siguientes.

$$
\begin{gathered}
M_{1}=\left(\eta_{20}+\eta_{02}\right) \\
M_{2}=\left(\eta_{20}-\eta_{02}\right)^{2}+4 \eta_{11}^{2} \\
M_{3}=\left(\eta_{30}-3 \eta_{12}\right)^{2}+\left(3 \eta_{21}-\eta_{03}\right)^{2} \\
M_{4}=\left(\eta_{30}+\eta_{12}\right)^{2}+\left(\eta_{21}+\eta_{03}\right)^{2} \\
M_{5}=\left(\eta_{30}-3 \eta_{12}\right)\left(\eta_{30}+\eta_{12}\right)\left[\left(\eta_{30}+\eta_{12}\right)^{2}\right. \\
\left.-3\left(\eta_{21}+\eta_{03}\right)^{2}\right]+\left(3 \eta_{21}-\eta_{03}\right)\left(\eta_{21}+\eta_{03}\right)\left[3\left(\eta_{30}+\eta_{12}\right)^{2}\right. \\
\left.-\left(\eta_{21}+\eta_{03}\right)^{2}\right] \\
M_{6}=\left(\eta_{20}-\eta_{02}\right)\left[\left(\eta_{30}+\eta_{12}\right)^{2}-\left(\eta_{21}+\eta_{03}\right)^{2}\right]+4 \eta_{11}\left(\eta_{30}+\eta_{12}\right)\left(\eta_{21}+\eta_{03}\right) \\
M_{7}=\left(3 \eta_{21}-\eta_{03}\right)\left(\eta_{21}+\eta_{03}\right)\left[3\left(\eta_{30}+\eta_{12}\right)^{2}\right. \\
\left.-\left(\eta_{21}+\eta_{03}\right)^{2}\right]-\left(\eta_{30}-3 \eta_{12}\right)\left(\eta_{21}+\eta_{03}\right)\left[3\left(\eta_{30}+\eta_{12}\right)^{2}-\left(\eta_{21}+\eta_{03}\right)^{2}\right]
\end{gathered}
$$

Donde $\eta_{p q}$ es el momento central de orden normalizado $(\mathrm{p}+\mathrm{q})$. Calculado como se muestra en la ecuación siguiente

$$
\eta_{p q}=\frac{\mu_{p q}}{\mu_{00}^{w}}, w=\frac{p+q}{2}+1, \forall p+q \geq 2
$$

Los elementos estadísticos del vector de características son: media, desviación estándar, curtosis y sesgo (ver ecuaciones a continuación). Estos valores se calculan sobre la distancia y reflexividad normalizadas a partir de los datos en bruto 3D LIDAR, generando ocho características.

$$
\begin{aligned}
\mu_{N D}^{(n)}=\frac{1}{n s} \sum_{i=1}^{n s} N D_{i}, & \mu_{R}^{(n)}=\frac{1}{n s} \sum_{i=1}^{n s} R_{i} \\
s_{N D}^{(n)}=\sqrt{\frac{1}{n s} \sum_{i=1}^{n s}\left(N D_{i}-\mu_{N D}^{(n)}\right)^{2},} & s_{R}^{(n)}=\sqrt{\frac{1}{n s} \sum_{i=1}^{n s}\left(R_{i}-\mu_{R}^{(n)}\right)^{2}} \\
k_{N D}^{(n)}=\frac{\frac{1}{n s} \sum_{i=1}^{n s}\left(N D_{i}-\mu_{N D}^{(n)}\right)^{4}}{\left(s_{N D}^{(n)}\right)^{4}}, & k_{R}^{(n)}=\frac{\frac{1}{n s} \sum_{i=1}^{n s}\left(R_{i}-\mu_{R}^{(n)}\right)^{4}}{\left(s_{R}^{(n)}\right)^{4}} \\
s k_{N D}^{(n)}=\frac{\frac{1}{n s} \sum_{i=1}^{n s}\left(N D_{i}-\mu_{N D}^{(n)}\right)^{3}}{\left(s_{N D}^{(n)}\right)^{3}}, & s k_{R}^{(n)}=\frac{\frac{1}{n s} \sum_{i=1}^{n s}\left(R_{i}-\mu_{R}^{(n)}\right)^{3}}{\left(s_{R}^{(n)}\right)^{3}}
\end{aligned}
$$

Siendo $\mu_{N D}^{(n)}, \mu_{R}^{(n)}, s_{N D}^{(n)}, s_{R}^{(n)}, k_{N D}^{(n)}, k_{R}^{(n)}, s k_{N D}^{(n)}$ y $s k_{R}^{(n)}$ los medios, las desviaciones estándar, la curtosis y la asimetría del conjunto de $n s$ mediciones de láser de distancia normalizada $N D_{i} \mathrm{y}$ reflexividad $\left(R_{i}\right)$ de una muestra $n$. 


\subsubsection{PASO 5: ALGORITMO DE APRENDIZAJE AUTOMÁTICO}

En este trabajo, usamos el rendimiento de tres algoritmos de ML (MLA) para detectar peatones: $\mathrm{k}$ Vecinos más cercanos (kNN), clasificador Naïve Bayes (NBC) y máquina de vectores de soporte (SVM). Estos algoritmos se probaron con diferentes parámetros de configuración (ver Tabla 12), y en todos los casos definimos dos clases de objetos: peatonal y no peatonal. kNN se probó con la distancia euclidiana y Mahalanobis con la normalización de datos; NBC se probó con Gauss y Kernel Smoothing Functions (KSF) sin normalización de datos; y SVM se probó con funciones lineales y cuadráticas con normalización de datos.

\begin{tabular}{|c|c|c|c|}
\hline Configuration & kNN & NBC & SVM \\
\hline method & Euclidean, Mahalanobis & Gauss, KSF & Linear, quadratic \\
\hline data normalisation & Yes & No & Yes \\
\hline metrics & LOOCV, ROC & LOOCV, ROC & LOOCV, ROC \\
\hline classes & 2 & 2 & 2 \\
\hline
\end{tabular}

Tabla 12. Parámetros de configuración de kNN, NBC y SVM

Estamos en la era de Big Data. Cada segundo se sube a Youtube una hora de video y sesenta billones de páginas web cada día; los nuevos sensores 3D LIDAR suministran cuatro mil millones de puntos por hora. Las técnicas de aprendizaje automático (ML) ofrecen soluciones para automatizar el análisis de Big Data. Una definición breve de ML es que se trata de un conjunto de métodos que pueden detectar automáticamente patrones en los datos. Entonces, podemos hacer uso de estos patrones descubiertos para hacer predicciones o realizar otros tipos de toma de decisiones bajo incertidumbre [92]. Los métodos ML generalmente se clasifican en dos tipos: aprendizaje supervisado y no supervisado.

El aprendizaje supervisado se basa en un conjunto de datos de entrenamiento etiquetados, que se utiliza para extraer un modelo matemático de los datos. Los métodos supervisados usan la distancia euclidiana, el teorema de Bayes, la regresión lineal, las distribuciones gaussianas, etc. para crear los modelos. Entre los métodos supervisados más conocidos están k-Nearest Neighbors, Naïve Bayes clasificadores, Artificial Neural Networks, o Support Vector Machines.

El aprendizaje no supervisado no utiliza un conjunto de datos de entrenamiento etiquetados porque no intenta aprender nada en particular. El aprendizaje no supervisado divide el conjunto de datos en grupos homogéneos, lo que se denomina agrupamiento. Se usa con bastante regularidad para la minería de datos. Por ejemplo, puede usarse para detectar patrones de comportamiento fraudulento o en el análisis de acciones del mercado. Los K-means, los modelos de mezcla o la agrupación jerárquica son enfoques para el aprendizaje no supervisado [93].

\subsection{RESULTADOS Y DISCUSION}

Dado que el algoritmo propuesto para la detección de peatones incluye un MLA, dividimos esta sección en dos partes: rendimiento y selección del MLA que detectará a los peatones en la nube de puntos generados por el LIDAR 3D, y el rendimiento del algoritmo general en un escenario real. Para ello, hemos definido tres escenarios. Los escenarios primero y segundo se han utilizado para extraer un conjunto de muestras de objetos peatonales y no peatonales de los datos generados por el LIDAR 3D. El primer escenario corresponde al Laboratorio de Visión Artificial y Vehículos Inteligentes de nuestro grupo de investigación (DSIE). En este escenario, se han tomado muestras de un peatón en diferentes ángulos y distancias, así como otros elementos paredes, obstáculos, vehículos, etc. El segundo escenario es un estacionamiento subterráneo, y se usó para capturar peatones y múltiples muestras de automóviles, motos, columnas, etc. El tercer escenario 
corresponde a un área de tráfico real alrededor de la Universidad Politécnica de Cartagena (ver Figura 48 (c)). Este último escenario se utilizó para probar el sistema en condiciones reales de tráfico.

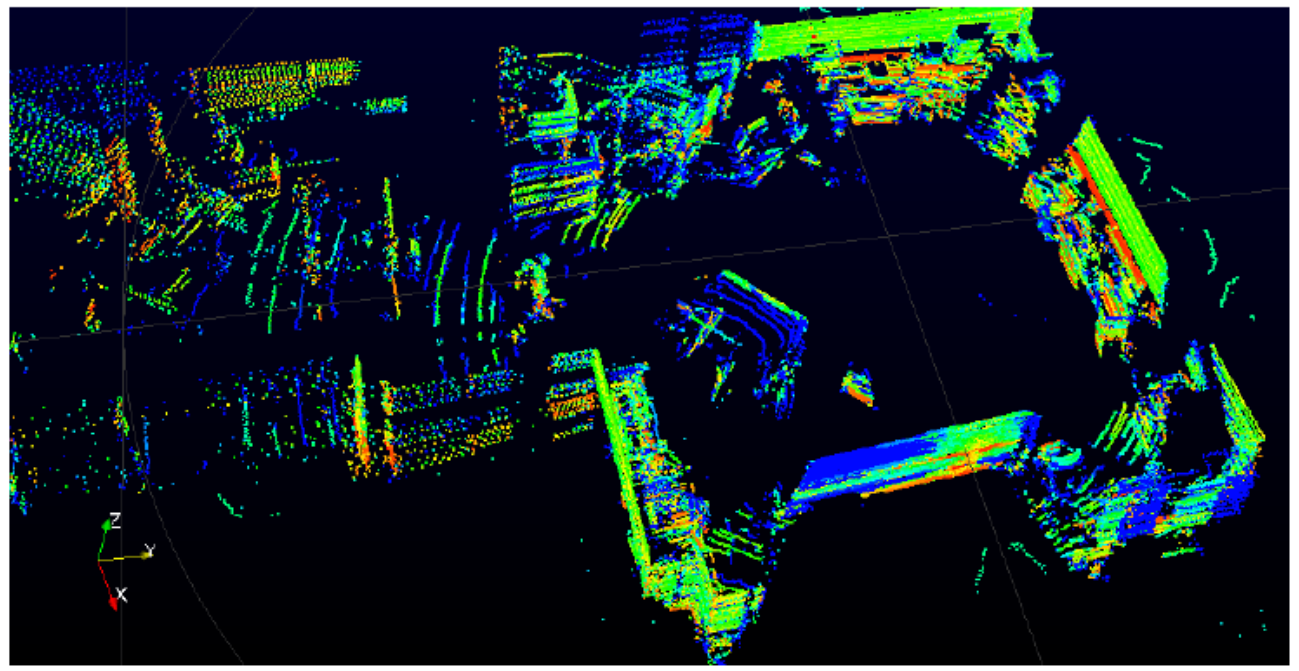

(a)

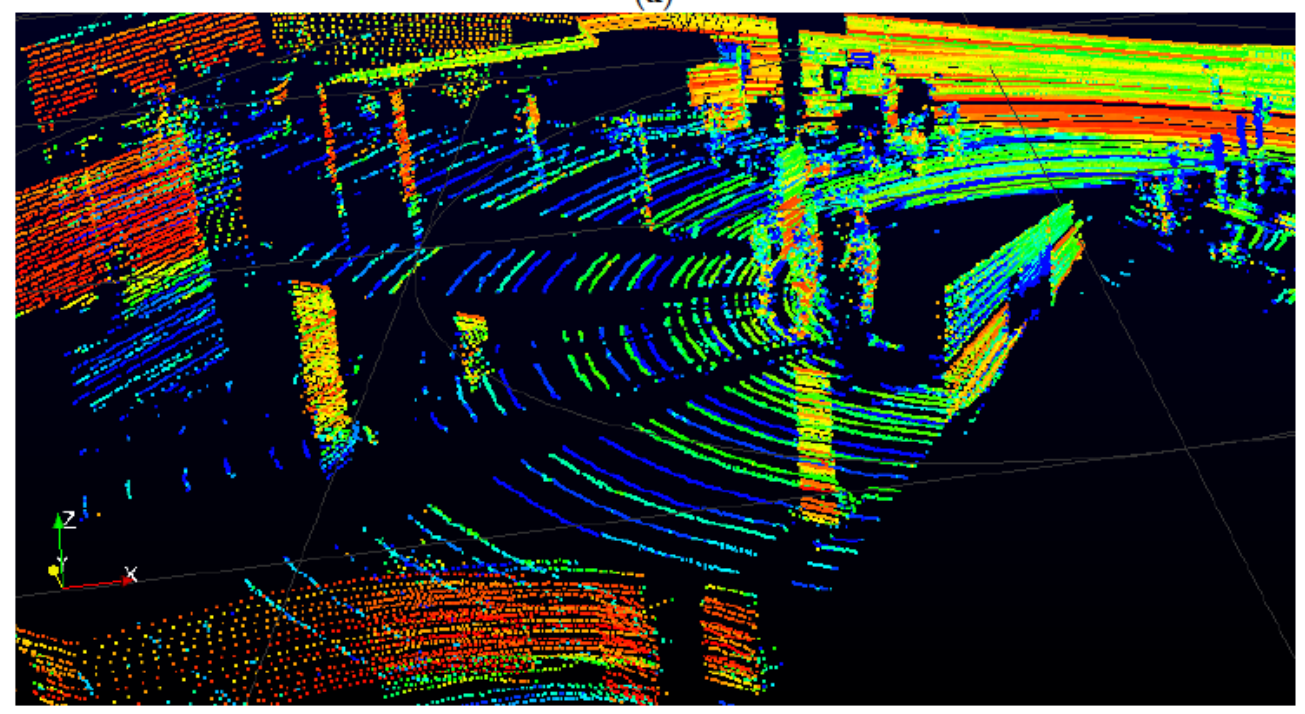

(b)

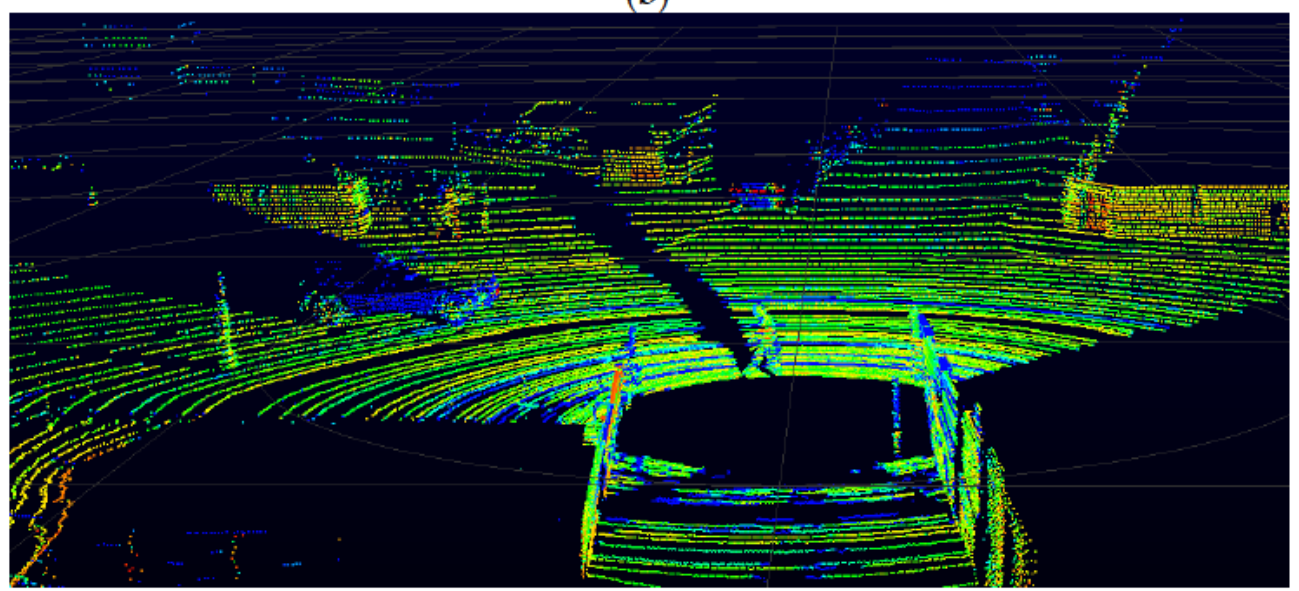

(c)

Figura 48. Tres escenarios: (a) Laboratorio de Visión Artificial y Vehículos Inteligentes; (b) estacionamiento subterráneo;(c) Escena de tráfico real. 


\subsubsection{RENDIMIENTO DE ALGORITMOS DE APRENDIZAJE AUTOMÁTICO}

Antes de seleccionar el MLA para la detección de peatones, primero necesitamos un conjunto de entrenamiento. Para capturar una muestra de puntos representativos de un peatón, entre los 1.3 millones de puntos generados por el LIDAR 3D en cada revolución (o cuadro), desarrollamos una herramienta de software en MATLAB. Esta herramienta, que se muestra en la Figura 49, carga datos láser almacenados previamente y permite a los usuarios seleccionar muestras de puntos de varios tamaños. Los valores predeterminados de la muestra se establecen en 100x100x200 centímetros. Los usuarios también pueden rotar el marco, filtrar los puntos según su distancia al origen de las coordenadas, así como modificar el tamaño de la muestra de selección. Una vez que se selecciona una muestra, el software almacena automáticamente los datos sin procesar de la muestra en un vector de características sin procesar, que incluye las coordenadas X, $\mathrm{Y}, \mathrm{Z}$, la distancia y la reflexividad, y permite al usuario indicar si la muestra contiene un peatón o no (es decir, su etiqueta). Del primer y segundo escenario, hemos extraído con la herramienta MATLAB 277 peatones y 1654 muestras no peatonales.

Hay varias métricas de evaluación que se pueden usar para evaluar el rendimiento de MLA. Calcular valores basados en la matriz de confusión, generar la curva de característica de funcionamiento del receptor (ROC), o realizar el método de validación cruzada de dejar uno fuera (LOOCV). Presentamos los resultados que hemos obtenido para todos los MLA considerados en esta sección. Las curvas LOOCV y ROC se han calculado bajo diferentes configuraciones de los MLA. La tabla también muestra los errores obtenidos después de aplicar el análisis de curvas LOOCV y ROC al conjunto de entrenamiento. Estas métricas nos permitieron seleccionar el MLA óptimo para el último paso del algoritmo de detección de peatones descrito en este capítulo.

La matriz de confusión presenta el número de muestras que fueron clasificadas correctamente por un MLA (verdaderos positivos (TP) y verdaderos negativos (TN)) contra aquellas que no eran (falsos positivos (FP) y falsos negativos (FN)). En el caso de los clasificadores binarios, como el que proponemos en este capítulo para detectar peatones, podemos calcular varios valores de la matriz de confusión, como la precisión; precisión, evalúa el poder predictivo del algoritmo); sensibilidad / especificidad; y puntaje F. Estos son normalmente los primeros indicadores considerados al evaluar un MLA, especialmente la precisión. Sin embargo, estos números por sí solos no pueden capturar completamente el rendimiento de un MLA, de la misma manera que la media no captura todas las características de un conjunto de datos.

La curva ROC caracteriza mejor el rendimiento de los MLA. Se calcula comparando la tasa de verdaderos positivos contra la tasa de falsos positivos en varios niveles de umbral. El área bajo la curva (AUC) también es un valor importante, ya que es una medida del poder de discriminación del clasificador, es decir, la capacidad del clasificador para clasificar correctamente los patrones presentados (peatones y no peatones en nuestro caso) [94], [95]. AUC también se puede interpretar como la probabilidad de que el clasificador asigne una puntuación más alta a un ejemplo positivo elegido al azar que a un ejemplo negativo elegido al azar. La Figura 49 muestra las curvas ROC calculadas para cada MLA con diferentes funciones de kernel. 


$$
\begin{gathered}
\text { Sensitivity }=\frac{T P}{T P+F N}, \quad \text { Specificity }=\frac{T N}{T N+F P} \quad(51,52) \\
\text { Precision }=\frac{T P}{T P+F P}, \\
\text { Accuracy }=\frac{(T P+T N)}{(T P+F P+F N+T N)} \\
\text { Fscore }=\frac{2 * T P}{2 * T P+F P+F N}
\end{gathered}
$$

LOOCV es un método simple de validación cruzada, que extrae una muestra del conjunto de entrenamiento y utiliza las muestras restantes para entrenar el MLA. Después de eso, clasifica la muestra que tomó y calcula el error de la clasificación. Este proceso se repite para todas las

\begin{tabular}{|c|c|c|c|c|c|c|c|}
\hline \multirow{2}{*}{\multicolumn{2}{|c|}{$\begin{array}{r}\text { MLA } \\
\text { Configuration }\end{array}$}} & \multicolumn{2}{|c|}{ kNN } & \multicolumn{2}{|c|}{$\overline{N B C}$} & \multicolumn{2}{|c|}{ SVM } \\
\hline & & Euclidean & Mahalanobis & $K S F$ & Gauss & Linear & Quadratic \\
\hline LOOCV & Error & 0.0653 & 0.0673 & 0.1361 & 0.6769 & 0.0528 & 0.0451 \\
\hline \multirow{6}{*}{$\underset{\bigcup}{己}$} & AUC & 0.9935 & 0.9916 & 0.9931 & 0.9317 & 0.9764 & 1.0000 \\
\hline & sensivity & 0.9764 & 0.9727 & 0.9304 & 0.2122 & 0.9758 & 1.0000 \\
\hline & specificity & 0.9205 & 0.9169 & 0.9891 & 0.9855 & 0.8194 & 1.0000 \\
\hline & precision & 0.9865 & 0.985907 & 0.9980 & 0.9887 & 0.9699 & 1.0000 \\
\hline & accuracy & 0.9684 & 0.964785 & 0.9388 & 0.3231 & 0.9533 & 1.0000 \\
\hline & Fscore & 0.9814 & 0.9793 & 0.9630 & 0.3494 & 0.9728 & 1.0000 \\
\hline
\end{tabular}
muestras. El error LOOCV se calcula luego como la media de los errores de la clasificación de cada muestra por sí mismo, lo que representa una medida de qué tan bien se ajusta el modelo a los datos suministrados. LOOCV es un método que es caro computacionalmente.

Tabla 13. Error de LOOCV y AUC para kNN, NBC, SVM

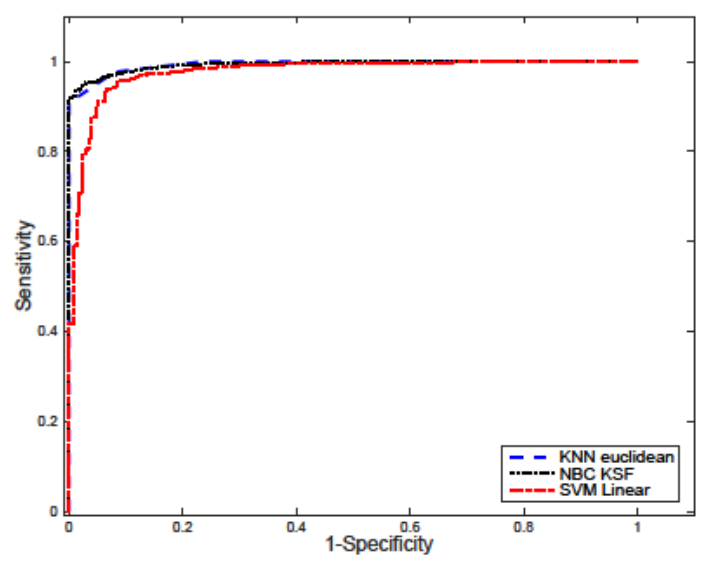

(a)

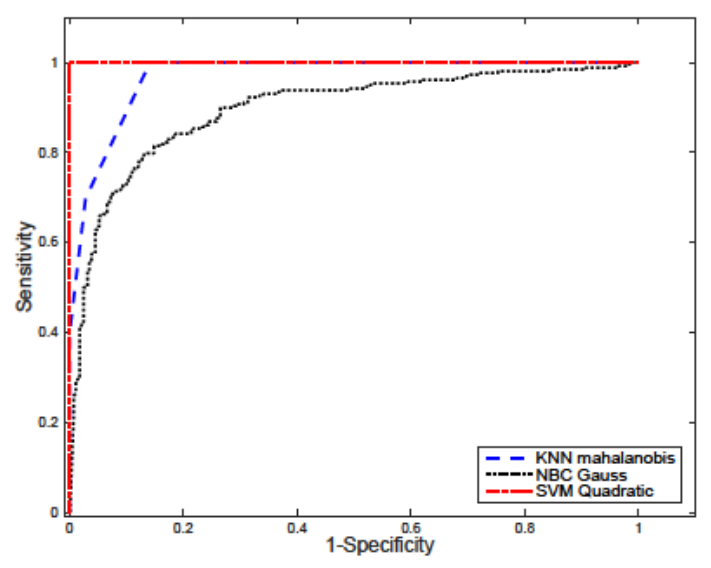

(b)

Figura 49. Resultados ROC: (a) clasificador kNN con distancia euclidiana, NBC con KSF y clasificador SVM con funciones lineales; (b) Clasificador kNN con distancia de Mahalanobis, NBC con kernel de Gauss y clasificador SVM con función polinomial cuadrátical.

Tanto el análisis LOOCV como el AUC muestran que el mejor ajuste del modelo de datos lo produce la SVM con kernel cuadrático, y los peores resultados se obtuvieron con la NBC con el kernel gaussiano. 


\subsubsection{RENDIMIENTO DEL ALGORITMO COMPLETO DE DETECCIÓN DE PEATONES}

Para determinar el mejor MLA que se usará en el algoritmo de detección de peatones, seleccionamos y probamos en el escenario 3 los algoritmos que tuvieron el error LOOCV más bajo, el AUC más alto y el Fscore más alto en la Tabla 14 Así, seleccionamos kNN con distancia euclidiana y SVM con funciones lineales y cuadráticas. Desde el escenario 3, hemos seleccionado siete cuadros de treinta metros cuadrados, y todos ellos se han dividido en muestras de 100x100x200 cm. La Tabla 14 muestra los resultados del rendimiento de los MLA seleccionados, obtenidos después de un cálculo manual de TP, FP, TN y FN. El número de peatones en cada cuadro se ha determinado manualmente (consulte la tercera columna de la Tabla 14).

\begin{tabular}{ccccccccccccccccc} 
& \multicolumn{1}{c}{ kNN - Eucliden } & \multicolumn{1}{c}{ SVM - Linear } & \multicolumn{1}{c}{ SVM - Quadratic } \\
\hline Frame & $\begin{array}{c}\text { Sam } \\
\text { ples }\end{array}$ & $\begin{array}{c}\text { Pedestrian in } \\
\text { the sample }\end{array}$ & TP & FP & TN & FN & TP & FP & TN & FN & TP & FP & TN & FN \\
\hline 1 & 100 & 1 & 1 & 7 & 92 & 0 & 1 & 2 & 97 & 0 & 1 & 14 & 85 & 0 \\
2 & 53 & 1 & 1 & 5 & 47 & 0 & 1 & 2 & 50 & 0 & 0 & 3 & 49 & 1 \\
3 & 47 & 1 & 1 & 4 & 42 & 0 & 1 & 1 & 45 & 0 & 1 & 10 & 36 & 0 \\
4 & 58 & 3 & 1 & 7 & 48 & 2 & 1 & 3 & 52 & 2 & 2 & 6 & 49 & 1 \\
5 & 79 & 4 & 3 & 5 & 70 & 1 & 3 & 3 & 72 & 1 & 4 & 9 & 66 & 0 \\
6 & 45 & 4 & 4 & 5 & 36 & 0 & 4 & 1 & 40 & 0 & 4 & 2 & 39 & 0 \\
7 & 103 & 2 & 2 & 9 & 92 & 0 & 2 & 3 & 98 & 0 & 2 & 6 & 95 & 0 \\
\hline Sum & 485 & 16 & 13 & 42 & 427 & 3 & 13 & 15 & 454 & 3 & 14 & 50 & 419 & 2
\end{tabular}

La Tabla 12 muestra los resultados métricos finales de los MLA probados. Como se muestra en la tabla, el mejor compromiso entre las métricas calculadas corresponde a la función lineal con SVM, que es el MLA que finalmente seleccionamos.

\begin{tabular}{cccc} 
& kNN - Eucliden & SVM - Linear & SVM - Quadratic \\
\hline sensivity & 0.8125 & 0.8125 & 0.8750 \\
specificity & 0.9104 & 0.9680 & 0.8934 \\
precision & 0.2364 & 0.4643 & 0.2188 \\
accuracy & 0.9072 & 0.9629 & 0.8928 \\
Fscore & 0.3662 & 0.5909 & 0.3500 \\
\hline
\end{tabular}

Tabla 15. Métricas del escenario 3

Las diferencias que encontramos entre los resultados teóricos de la Tabla 14 (donde la SVM con un kernel cuadrático muestra los mejores resultados) con respecto a los resultados del escenario 3 (que se muestra en la Tabla 15), se deben al "problema de sobreajuste". Un MLA presenta un ajuste excesivo cuando es capaz de aprender todas las muestras proporcionadas en el conjunto de capacitación por sí mismo, pero no puede generalizar a nuevos datos. Dados los resultados que se muestran en la Tabla 14, esperamos que SVM con kernel cuadrático logre un 100\% de éxito al clasificar muestras nuevas y desconocidas, pero su rendimiento disminuye en todas las métricas, como se muestra en la última columna de la Tabla 15, siendo incluso más bajo que otros MLAs. El problema de sobreajuste puede ser causado por muchos factores, uno de los cuales es un bajo número de muestras de entrenamiento. En nuestro caso, probamos un número diferente de muestras de entrenamiento (de 200 a 2000 con un paso de 200), pero el problema persistió. Por esta razón, finalmente decidimos usar el SVM lineal como MLA para la detección de peatones, dentro del módulo de interpretación de la escena en el CICar. 
La Figura 50 muestra el resultado obtenido al ejecutar el algoritmo de detección de peatones en el cuadro seis, donde hay tres peatones. En este marco, el algoritmo evaluó cuarenta y cinco muestras. Como se muestra en la imagen, el algoritmo detectó cuatro peatones (un falso positivo).

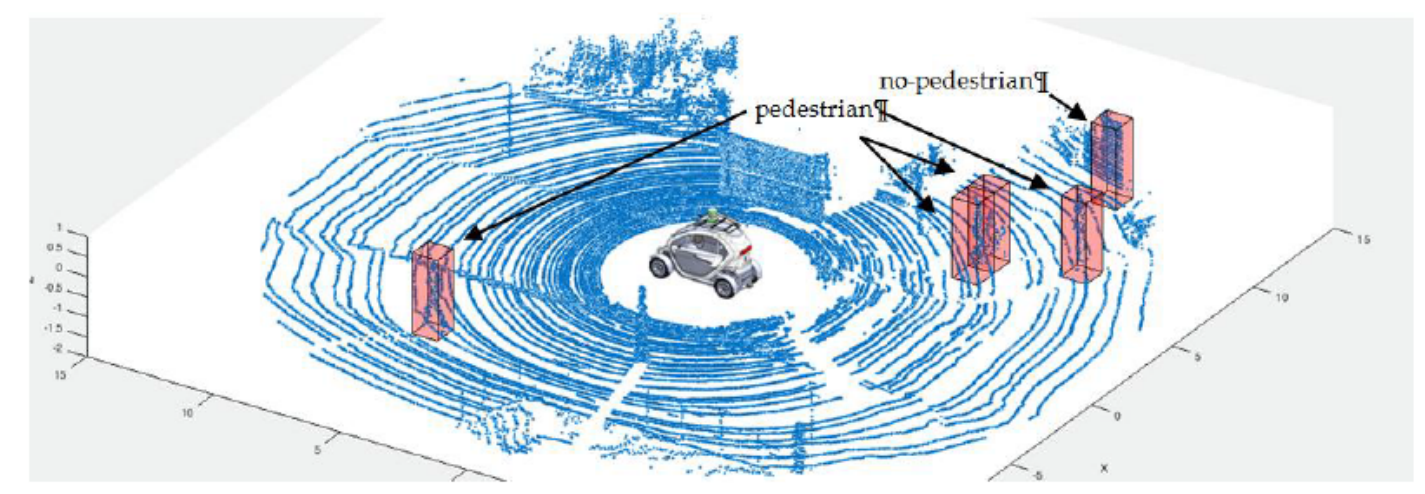

Figura 50. Algoritmo de detección de peatones en área de tráfico real.

La Tabla 16 muestra una comparación del método propuesto con los cinco métodos, que usan LIDAR 3D de alta definición para llevar a cabo la detección de peatones.

\begin{tabular}{|c|c|c|c|c|}
\hline Autores / año & MLA & Gráfico & Numérico & Rendimiento \\
\hline Proposed 2016 & Linear SVM & Curvas ROC & $\begin{array}{l}\text { LOOCV, AUC, sensibilidad, especificidad, } \\
\text { precisión, precisión, Fscore }\end{array}$ & $\begin{array}{c}0.0528,0.9764,0.9758,0.8194, \\
0.9699,0.9533,0.9728\end{array}$ \\
\hline Premebida 2014 [96] & $\begin{array}{l}\text { Deformable Part-based Model } \\
\text { (DPM) }\end{array}$ & $\begin{array}{l}\text { Curca Precisión Recall de las áreas } \\
\text { del indefinido peatón }\end{array}$ & Los autores reportan una media & 0.3950 \\
\hline Spinello 2010 [97] & $\begin{array}{l}\text { Multiple AdaBoost } \\
\text { classifiers } \\
\end{array}$ & $\begin{array}{c}\text { Curva Precisión Recall, ratio iguales } \\
\text { errores (EER) }\end{array}$ & Los autores reportan una media & 0.7760 \\
\hline $\begin{array}{c}\text { Navarro-Serment } \\
2010[98] \\
\end{array}$ & Two SVMs in cascade & Precisión Recall y curcaROC & Estimación AUC & 0.8500 \\
\hline Ogawa 2011 [99] & Interacting Multiple Model filter & Tasa reconocimiento & Los autores reportan una media & 0.8000 \\
\hline Kidono 2011 [100] & SVM & Curva ROC & Estimación AUC & 0.9000 \\
\hline
\end{tabular}

Tabla 16. Comparación del método propuesto con otros autores.

La comparación se realiza en términos de implementación de MLA, la métrica utilizada (valores gráficos y numéricos) y rendimiento. Queremos resaltar que existe una falta de homogeneidad en el informe de las estadísticas de desempeño en los trabajos revisados y una ausencia de resultados numéricos en muchos de ellos. Este hecho hace que la comparación minuciosa de los resultados sea casi imposible. Sin embargo, hemos estimado el AUC de los trabajos [98], [100] y usamos la métrica Fscore para comparar con los trabajos donde los autores informan valores medios ad-hoc, ya que Fscore se define como el armónico medio de precisión y sensibilidad (o recuerdo) y establece la relación entre ellos. Como se muestra en la Tabla 16, el AUC de los autores [98], [100] es inferior al método propuesto y la media de rendimiento informada por [96] [97] [99] es menor que el Fscore obtenido por el método propuesto. 


\section{CAPITULO 7}

\section{CONCLUSIONES Y TRABAJOS FUTUROS}

\subsection{APORTACIONES Y CONCLUSIONES}

El rápido avance tecnológico de la sociedad actual ha propiciado un elevado grado de automatización de tareas impensables de automatizar hace apenas unos años y ha llevado a la aspiración de automatizar las tareas de conducción de los vehículos que nos facilitan la movilidad.

La denominada conducción autónoma afecta a una potente industria desde los puntos de vista económico, laboral y social; una industria en constante evolución en la que el vehículo autónomo supondrá -está de hecho suponiendo- una enorme revolución. Pero es que, además, este cambio nos afectará profundamente como sociedad, puesto que cambiará de forma radical nuestras pautas y conceptos de movilidad e, incluso, nuestros hábitos sobre la propiedad de vehículos. Parece indudable que esta transformación traerá mejoras en aspectos tan importantes como el cuidado del medioambiente, la reducción de la siniestralidad, la optimización de los tiempos de desplazamiento, etc.

Dicho lo anterior, no es de extrañar que tanto la mayoría de fabricantes de automóviles como otros actores del sector auxiliar estén inmersos en una verdadera carrera tecnológica que produce resultados continuamente, aportando sistemas que aumentan día a día el grado de autonomía de los nuevos automóviles.

En 2020 el vehículo autónomo, en versiones comerciales para el uso en ciudades, presenta un nivel 2 de conducción autónoma; en nuestra opinión, y basándonos en predicciones de reconocidos expertos en conducción autónoma, todavía estamos lejos de disfrutar de forma más o menos generalizada de vehículos con nivel 5 de autonomía en nuestra red viaria. Aunque la tecnología hardware y de sensores está ya en un nivel maduro, todavía hay un largo camino que recorrer en aspectos como el reconocimiento de escenas y situaciones o la gestión del comportamiento en entornos de tráfico real. Por no mencionar los aspectos legales, en los cuales se necesitará todavía un avance considerable.

La conducción autónoma se aborda desde una dimensión prismática, en la que cada cara del prisma representa un componente de un complejo puzzle cuyas piezas han de encajar para conseguir el objetivo. 
En la presente tesis se han abordado solamente algunos de los aspectos de la conducción autónoma, cuyas conclusiones hemos considerado oportuno presentarlas en cuatro partes: (1) la robotización mecánica del vehículo, (2) la sensorización del vehículo, (3) el desarrollo de un método planificación de ruta y (4) el desarrollo de un novedoso algoritmo para la detección peatones.

(1) se han robotizado mecánicamente los sistemas principales (volante, freno, acelerador y sentido de marcha) para lograr la conducción autónoma de un vehículo comercial Renault Twizy. La elección de este modelo comercial es debida a su bajo coste y accesibilidad mecánica, propulsión $100 \%$ eléctrica y bajo mantenimiento. Con ello se diseñado e implementado una plataforma viable para la conducción autónoma, concluyendo que:

- para la automatización, se ha implementado soluciones mecánicas de fácil instalación, con la menor interferencia y modificación de los sistemas originales del vehículo. Se exponen cálculos de esfuerzos para una selección óptima de motoreductores y engranajes. Esta robotización permite el control y guiado de trayectorias, a través, de una planificación previa de la ruta.

- se ha estudiado el teorema de Lyapunov como algoritmo que ayuda a controlar la velocidad del vehículo y el ángulo de giro del volante, parámetros necesarios para lograr alcanzar los puntos objetivo a seguir en la ruta programada. Estos sistemas controlados, seguirán rutas establecidas a partir de teoría geométrica como es la configuración Ackerman, muy extendida en el ámbito de la conducción automática.

(2) el sistema de percepción resulta crucial para dotar de reconocimiento de la escena en un entorno cambiante, además del autoposicionamiento del vehículo autónomo.

La tecnología de sensorización existente actualmente en el mercado, ofrece diversidad de medios para captar señales, formas, objetos, colores, etc. y su ubicación relativa. Estos sistemas son el eslabón central determinante para conectar la automatización o robotización con el control de trayectorias.

- se han instalado una gran variedad de sensores de diferentes tecnologías y métodos de captación. Estos sensores arrojan un enorme volumen de datos, con la consecuente labor de gestionar, clasificar y fusionar. Un método conocido es el Filtro de Kalman, que permite la fusión de datos estadísticamente óptimos para mejorar los datos globalmente.

- se ha estudiado la variedad de software para la simulación de entornos virtuales para ensayo y aprendizaje del entorno exterior, a través de los sensores instalados en vehículo autónomo, que reducen costos de investigación.

(3) el Vehículo Inteligente (VI) jugará un papel central en los llamados Sistemas Inteligentes de Transporte. Por este motivo, se dedica un gran esfuerzo de investigación al desarrollo de algoritmos y estrategias de control para conducción autónoma. Es fundamental, por tanto, disponer de plataformas de conducción reales listas para implementar y pruebe estos algoritmos y estrategias de control.

- se ha desarrollado e implementado sistemas abiertos de bajo y alto nivel para el vehículo autónomo: el CICar. La arquitectura de bajo nivel se presenta en detalle, y comprende una descripción de todos los componentes de hardware, sensores y comunicación a bordo. Además, se describe la arquitectura de alto nivel, que reúne a los sistemas que dotan al vehículo de un cierto grado de inteligencia.

- a diferencia de otras plataformas presentadas, que utilizan sistemas de control propios, se ha propuesto un enfoque basado en conocidas plataformas de desarrollo de hardware y software para fines de control: arquitectura RIO y LabVIEW. Esto significa que el 
desarrollo de software actual, para la conducción autónoma, se puede transferir fácilmente para controlar acciones en cualquier vehículo equipado con el hardware de control de propósito general propuesto para el CICar. La infraestructura de detección incluye cámaras ToF para vigilancia lateral. Esta tecnología de detección ofrece ventajas frente al láser 2D actual, ya que se consigue una mejor caracterización de la escena, ofreciendo así mejores capacidades tales como: detección de objetos 3D con una alta velocidad de influencia de las condiciones de luz y sombras, y costo aceptable.

- se ha mostrado un caso de estudio donde la implementación de un PPS (sistema de planificación de rutas) en la plataforma CICar. El PPS implementado utiliza un mapa para una ruta global y un planificador local para resolver los problemas de tráfico habituales. El planificador global ha sido resuelto mediante el desarrollo de un nuevo algoritmo heurístico basado en una búsqueda de puntos de cruce (SCP) usando mapas binarios. El algoritmo SCP ha mejorado la flexibilidad, el tiempo de cálculo y el rendimiento en el cálculo de rutas globales. Además, también se ha presentado un planificador local en este trabajo, que implementa un generador de trayectoria basado en curvas de Bézier, que suministra alta flexibilidad durante la resolución de situaciones imprevistas. El generador de trayectoria permite el ajuste de: (a) el número de trayectorias posibles a generar; (b) una distancia de seguridad; (c) un criterio para seleccionar la trayectoria óptima. El vehículo autónomo CICar ofrece una plataforma comprensible y confiable para desarrollo y prueba de tecnología de conducción autónoma.

(4) se ha presentado un enfoque de aprendizaje automático para la detección de peatones para vehículos autónomos utilizando datos de rango 3D de alta definición, ya que la detección de peatones ha sido identificada como una de las tareas más críticas para vehículos autónomos. El algoritmo de aprendizaje automático utilizará para discriminar a los peatones de otros objetos en sus alrededores. Las proyecciones axonométricas de muestras de puntos de nube se utilizan para crear imágenes binarias, lo que nos ha permitido emplear algoritmos de visión por computadora para calcular parte de dicho vector de características. Este se compone de: características de forma, momentos invariantes y características estadísticas de distancias y reflexividad del LIDAR 3D.

- se ha realizado un análisis exhaustivo del rendimiento de tres algoritmos de aprendizaje automático diferentes: k-Vecinos más cercanos (kNN), clasificador Naïve Bayes (NBC) y vector de soporte máquina (SVM). Cada algoritmo se entrenó con un conjunto de entrenamiento que incluye la herramienta 277 peatones y 1654 muestras sin peatones. Se utilizaron análisis LOOCV y ROC para detectar el mejor algoritmo a utilizar para detección de peatones. El algoritmo propuesto ha sido probado en escenarios de tráfico reales con 16 muestras de peatones y 469 muestras de no peatones. Los resultados obtenidos se utilizaron para validar los resultados teóricos obtenidos. Se concluye que el método propuesto obtiene mejores resultados de desempeño en todos los casos. La alta tasa de éxito y la escalabilidad de los algoritmos de aprendizaje automático permitirán la detección de diferentes objetos durante la navegación del vehículo autónomo. 


\subsection{TRABAJOS FUTUROS}

Los trabajos futuros expuestos a continuación han sido propuestos con el fin de consolidar las líneas principales de investigación de la presente tesis.

- se está trabajando para aumentar la base de datos de muestras de nubes de puntos, así como en la creación de máquinas de aprendizaje capaces de detectar otros objetos en escenas diferentes, como son bicicletas, automóviles, señales de tráfico y luces, etc. La fusión de sensores también es muy importante, y por lo tanto se está muy interesado en utilizar la información de las cámaras montadas en nuestro coche. Además, dado que los algoritmos son computacionalmente costosos, se está desarrollando código paralelizable para aumentar la velocidad del algoritmo.

- se ampliará la sensorización del vehículo con nuevos radares de última generación, cámaras térmicas, etc..., con software propio de identificación de peatones. Un aspecto a mejorar es el incremento en la robustez y eficacia del sistema, orientado a la toma decisiones morales y éticas como lo hacen los humanos, decisiones de vida o muerte.

- se implementará nuevos sensores, software y aplicaciones para aumentar el campo de trabajo en el vehículo autónomo. Se adquirió otro vehículo comercial Renault Zoe de 5 plazas, completamente eléctrico, para nuevos desarrollos e instalación de parte de los sensores estudiados, así como mejora de las aplicaciones y algoritmos. 


\section{REFERENCIA BIBLIOGRÁFICA}

[1] S. Cars, "VEHÍCULOS AUTÓNOMOS Y DERECHOS DAÑOS," pp. 55-73, 2018.

[2] S. Han, Y. Byen, J. Baek, T. K. An, S.-G. Lee, and H. J. Park, "An optimal automatic train operation (ATO) control using genetic algorithms (GA)," TENCON 99. Proc. IEEE Reg. 10 Conf., vol. 1, pp. 360-362, 1999, doi: 10.1109/TENCON.1999.818425.

[3] European Union, "Experience and recommendations," no. 314190, p. 42, 2016.

[4] F. Jiménez, INTELLIGENT VEHICLES. 2017.

[5] M. H. . . Sebastian Thrun, Mike Montemerlo, Hendrik Dahlkamp, David Stavens, Andrei Aron, James Diebel, Philip Fong, John Gale, "Stanley: The Robot That Won the DARPA Grand Challenge," p. S. 180, 2012, doi: BF00813859.

[6] U. Ozguner, C. Stiller, and K. Redmill, "Systems for Safety and Autonomous Behavior in Cars: The DARPA Grand Challenge Experience," Proc. IEEE, vol. 95, no. 2, pp. 397-412, Feb. 2007, doi: 10.1109/JPROC.2006.888394.

[7] D. Learning and D. Cars, "Lecture 2 of course 6.S094: Deep Learning for Self - Driving Cars (2018 version).," p. 2018, 2018.

[8] "R. Dqg, H. Ri, D. Q. Fnhupdq, and E. Q. L. Dwlrq, "07075182 (1)," vol. 1, pp. 5-10, 2009.

[9] S. Cars, "VEHÍCUlOS AUTÓNOMOS Y DERECHOS DAÑOS," pp. 65, 2018.

[10] J. Jimenez, "Simulación de vehículos eléctricos ligeros," p. 31, 2015.

[11] A. Furda and L. Vlacic, "Enabling safe autonomous driving in real-world city traffic using Multiple Criteria decision making," IEEE Intell. Transp. Syst. Mag., vol. 3, no. 1, pp. 4-17, 2011, doi: 10.1109/MITS.2011.940472.

[12] J. Jimenez, "Simulación de vehículos eléctricos ligeros," p. 51, 2015.

[13] European Union, "Experience and recommendations," no. 314190, p. 22, 2016.

[14] Y. Q. Chen, M. S. Nixon, and D. W. Thomas, "Statistical geometrical features for texture classification," Pattern Recognit., vol. 28, no. 4, pp. 537-552, 2005.

[15] J. Jimenez, "Simulación de vehículos eléctricos ligeros," p. 121, 2015.

[16] C. H. Chan, "Fabric defect detection by Fourier analysis," IEEE Trans. Ind. Appl., vol. 36, no. 5 , pp. 1267-1276, 2000, doi: 10.1109/28.871274.

[17] M. E. Bronner et al., "Re s ear ch | r e p o r t s," vol. 1080, no. 2013, pp. 1-5, 2015.

[18] J. Gogoll and J. F. Müller, "Autonomous Cars: In Favor of a Mandatory Ethics Setting," Sci. Eng. Ethics, vol. 23, no. 3, pp. 681-700, 2017, doi: 10.1007/s11948-016-9806-x.

[19] European Union, "Experience and recommendations," no. 314190, p. 32, 2018.

[20] N. Goodall, "Ethical Decision Making During Automated Vehicle Crashes," Transp. Res. Rec. J. Transp. Res. Board, vol. 2424, no. 2424, pp. 58-65, 2014, doi: 10.3141/2424-07.

[21] M. A. Wächter et al., "Human decisions in moral dilemmas are largely described by Utilitarianism: virtual car driving study provides guidelines for ADVs," arXiv, 2017.

[22] D. Das and M. A. Civil, "On-Street Parking Demand Estimation in Urban CBD using FI and CF Model: A Case Study - Kolkata, India," Indian J. Sci. Technol., vol. 10, no. 12, pp. 1-11, 2017, doi: 10.17485/ijst/2017/v10i12/104029.

[23] European Union, "Experience and recommendations," no. 314190, p. 52, 2016.

[24] W. Zhang and S. Guhathakurta, "Parking Spaces in the Age of Shared Autonomous Vehicles: How Much Parking Will We Need and Where?," Transp. Res. Board 96th Annu. Meet., pp. 17-05399, 2017.

[25] J. Liu, P. Fellow, K. M. Kockelman, P. M. Boesch, and F. Ciari, "Tracking a system of shared autonomous vehicles across the austin, texas network using agent-based simulation," Transportation (Amst)., 2016, doi: 10.1007/s11116-017-9811-1. 
[26] J. Jimenez, "Simulación de vehículos eléctricos ligeros," p. 131, 2015.

[27] "Universidad politécnica de cartagena," 2018.

[28] F. D. Hobbs, "Traffic planning and engineering." 1898.

[29] A. Yenkins, "Jenkins, A., 2007. Remote Sensing Technology for Automotive Safety.pdf.".

[30] J. Jimenez, "Simulación de vehículos eléctricos ligeros," p. 111, 2012.

[31] R. Dqg, H. Ri, D. Q. Fnhupdq, and E. Q. L. Dwlrq, “07075182 (1)," vol. 1, pp. 5-10, 2009.

[32] T. Dalgleish et al., [No Title ], vol. 136, no. 1. 2007.

[33] C. A. R. Sim, "A Review On Automatic Steering Control Using Nested PID Controller."

[34] G. Tagne, R. Talj, and A. Charara, "Higher-Order Sliding Mode Control for Lateral Dynamics of Autonomous Vehicles, with Experimental Validation," 2013 IEEE Intell. Veh. Symp., no. Iv, pp. 678-683, 2013, doi: 10.1109/IVS.2013.6629545.

[35] "Nonlinear Control of Wheeled Mobile Robots," vol. 262, no. 0, p. 6221, 2001.

[36] A. Bicchi, "Unicycle-like Vehicles via Lyapunov Techniques," no. March, pp. 27-35, 1995.

[37] K. Bimbraw, "Autonomous cars: Past, present and future: A review of the developments in the last century, the present scenario and the expected future of autonomous vehicle technology," ICINCO 2015 - 12th Int. Conf. Informatics Control. Autom. Robot. Proc., vol. 1, pp. 191-198, 2015, doi: 10.5220/0005540501910198.

[38] A. Bavelloni, M. Piazzi, M. Raffini, I. Faenza, and W. L. Blalock, "Prohibitin 2: At a communications crossroads," IUBMB Life, vol. 67, no. 4, pp. 239-254, 2015, doi: 10.1002/iub.1366.

[39] C. Liang, Y. Li, and J. W. Luo, "A Novel Method to Detect Functional microRNA Regulatory Modules by Bicliques Merging," IEEE/ACM Trans. Comput. Biol. Bioinforma., vol. 13, no. 3, pp. 549-556, 2016, doi: 10.1109/TCBB.2015.2462370.

[40] B. Eissfeller, G. Ameres, V. Kropp, and D. Sanroma, "Performance of GPS , GLONASS and Galileo," Eisfeller al., pp. 185-199, 2007.

[41] H. Herrmann and H. Bucksch, "Differential GPS," Dict. Geotech. Eng. Geotech., pp. 375375, 2014, doi: 10.1007/978-3-642-41714-6_41898.

[42] R. Borraz, P. Navarro, C. Fernández, and P. Alcover, "Cloud Incubator Car: A Reliable Platform for Autonomous Driving," Appl. Sci., vol. 8, no. 2, p. 303, 2018, doi: 10.3390/app8020303.

[43] S. Sun, C. Li, and A. H. Paterson, "In-field high-throughput phenotyping of cotton plant height using LiDAR," Remote Sens., vol. 9, no. 4, pp. 1-21, 2017, doi: 10.3390/rs9040377.

[44] "Kim, J., Lee, M., 2014. Robust lane detection based on convolutional neural network and random sample consensus. Neural Information Processing, ICONIP 2014. Lecture Notes in Computer Science. Springer, pp. 454 _ 461.," p. 2014, 2014.

[45] R. Lange and P. Seitz, "Solid-state time-of-flight range camera," IEEE J. Quantum Electron., vol. 37, no. 3, pp. 390-397, 2001, doi: 10.1109/3.910448.

[46] "Li, L. Time-of-Flight Camera-An Introduction; Technical White Paper; Texas Instruments Dallas, TX, USA.pdf." .

[47] P. Navarro, C. Fernández, R. Borraz, and D. Alonso, "A Machine Learning Approach to Pedestrian Detection for Autonomous Vehicles Using High-Definition 3D Range Data," Sensors, vol. 17, no. 1, p. 18, 2016, doi: 10.3390/s17010018.

[48] A. Yenkins, "Jenkins, A., 2007. Remote Sensing Technology for Automotive Safety.pdf." .

[49] M. Conner, "Conner, M., 2011. Automobile sensors may usher in self-driving cars.pdf." .

[50] C. Piñana-Díaz, R. Toledo-Moreo, F. Javier Toledo-Moreo, and A. Skarmeta, "A two-layers based approach of an enhanced-map for urban positioning support," Sensors (Switzerland), vol. 12, no. 11, pp. 14508-14524, 2012, doi: 10.3390/s121114508.

[51] T. Pastore, H. Everett, K. Bonner, and S. AND, "Mobile robots for outdoor security applications," Meet. Robot., pp. 1-16, 1999.

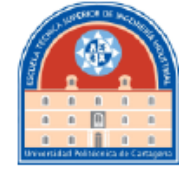


[52] H. W. Sorenson, "Least-squares estimation- from Gauss to Kalman.pdf," pp. 63-68, doi: 10.1109/MSPEC.1970.5213471.

[53] "Universidad politécnica de cartagena," 2019.

[54] C. Katrakazas, M. Quddus, W. H. Chen, and L. Deka, "Real-time motion planning methods for autonomous on-road driving: State-of-the-art and future research directions," Transp. Res. Part C Emerg. Technol., vol. 60, pp. 416-442, 2015, doi: 10.1016/j.trc.2015.09.011.

[55] S. Zhang, W. Deng, Q. Zhao, H. Sun, and B. Litkouhi, "Dynamic trajectory planning for vehicle autonomous driving," Proc. - 2013 IEEE Int. Conf. Syst. Man, Cybern. SMC 2013, pp. 4161-4166, 2013, doi: 10.1109/SMC.2013.709.

[56] C. Urmson, "Autonomous Driving in Urban Environments: Boss and the Urban Challenge," Journal of Field Robotics, vol. 25, no. 8. pp. 426-466, 2008, doi: 10.1002/rob.

[57] J. G. Mooney and E. N. Johnson, "A Comparison of Automatic Nap-of-the-earth Guidance Strategies for Helicopters," J. F. Robot., vol. 33, no. 1, pp. 1-17, 2014, doi: 10.1002/rob.

[58] E. Galceran, A. G. Cunningham, R. M. Eustice, and E. Olson, "Multipolicy decision-making for autonomous driving via changepoint-based behavior prediction: Theory and experiment," Auton. Robots, vol. 41, no. 6, pp. 1367-1382, 2017, doi: 10.1007/s10514-017-9619-z.

[59] M. Czubenko, Z. Kowalczuk, and A. Ordys, "Autonomous Driver Based on an Intelligent System of Decision-Making," Cognit. Comput., vol. 7, no. 5, pp. 569-581, 2015, doi: $10.1007 / \mathrm{s} 12559-015-9320-5$.

[60] B. Chen and H. H. Cheng, "A review of the applications of agent technology in traffic and transportation systems," IEEE Trans. Intell. Transp. Syst., vol. 11, no. 2, pp. 485-497, 2010, doi: 10.1109/TITS.2010.2048313.

[61] R. Abdullah, A. Hussain, K. Warwick, and A. Zayed, "Autonomous intelligent cruise control using a novel multiple-controller framework incorporating fuzzy-logic-based switching and tuning," Neurocomputing, vol. 71, no. 13-15, pp. 2727-2741, 2008, doi: 10.1016/j.neucom.2007.05.016.

[62] T. Belker, M. Beetz, and A. B. Cremers, "Learning action models for the improved execution of navigation plans," Rob. Auton. Syst., vol. 38, no. 3-4, pp. 137-148, 2002, doi: 10.1016/S0921-8890(02)00163-X.

[63] D. Chakraborty, W. Vaz, and A. K. Nandi, "Optimal driving during electric vehicle acceleration using evolutionary algorithms," Appl. Soft Comput. J., vol. 34, pp. 217-235, 2015, doi: 10.1016/j.asoc.2015.04.024.

[64] A. G. Cunningham, E. Galceran, R. M. Eustice, and E. Olson, "MPDM: Multipolicy decision-making in dynamic, uncertain environments for autonomous driving," Proc. - IEEE Int. Conf. Robot. Autom., vol. 2015-June, no. June, pp. 1670-1677, 2015, doi: 10.1109/ICRA.2015.7139412.

[65] S. Behere and M. Törngren, "A Functional Architecture for Autonomous Driving," Proc. First Int. Work. Automot. Softw. Archit. - WASA '15, pp. 3-10, 2015, doi: $10.1145 / 2752489.2752491$.

[66] Y. Kuwata, J. Teo, G. Fiore, S. Karaman, E. Frazzoli, and J. P. How, "Real-time motion planning with applications to autonomous urban driving," IEEE Trans. Control Syst. Technol., vol. 17, no. 5, pp. 1105-1118, 2009, doi: 10.1109/TCST.2008.2012116.

[67] A. Furda and L. Vlacic, Multiple criteria-based real-time decision making by autonomous city vehicles, vol. 7, no. PART 1. IFAC, 2010.

[68] A. Furda and L. Vlacic, "Enabling safe autonomous driving in real-world city traffic using Multiple Criteria decision making," IEEE Intell. Transp. Syst. Mag., vol. 3, no. 1, pp. 4-17, 2011, doi: 10.1109/MITS.2011.940472.

[69] B. Flemming, V. Gill, P. Godsmark, and B. Kirk, "Automated Vehicles: The Coming of the Next Disruptive Technology," Conf. Board Canada, no. January, 2015. 
[70] Peter E. Hart, Nils J. Nilsson, and Bertram Raphael, "Formal Basis for the Heuristic Determination eijj ," Syst. Sci. Cybern., no. 2, pp. 100-107, 1968.

[71] E. W. Dijkstra, "A note on two problems in connexion with graphs," Numer. Math., vol. 1, no. 1, pp. 269-271, 1959, doi: 10.1007/BF01386390.

[72] C. Harris and M. Stephens, "A Combined Corner and Edge Detector," Procedings Alvey Vis. Conf. 1988, pp. 23.1-23.6, 1988, doi: 10.5244/C.2.23.

[73] S. Biswas and B. C. Lovel, "Bezier and Splines in Image Processing and Machine Vision," Heal. (San Fr., p. 246, 2008.

[74] P. Dollar, C. Wojek, B. Schiele, and P. Perona, "Pedestrian detection: An evaluation of the state of the art," IEEE Trans. Pattern Anal. Mach. Intell., vol. 34, no. 4, pp. 743-761, 2012, doi: 10.1109/TPAMI.2011.155.

[75] R. Benenson, M. Omran, J. Hosang, and B. Schiele, "Ten years of pedestrian detection, what have we learned?," Lect. Notes Comput. Sci. (including Subser. Lect. Notes Artif. Intell. Lect. Notes Bioinformatics), vol. 8926, pp. 613-627, 2015, doi: 10.1007/978-3-31916181-5_47.

[76] J. F. Man, H. Y. Lin, Q. Q. Li, and X. B. Wen, "Segmentation and Tracking of Human in Crowded Environments," Adv. Mater. Res., vol. 255-260, no. 7, pp. 2281-2285, 2011, doi: 10.4028/www.scientific.net/AMR.255-260.2281.

[77] J. Satake, M. Chiba, and J. Miura, "Visual person identification using a distance-dependent appearance model for a person following robot," Int. J. Autom. Comput., vol. 10, no. 5, pp. 438-446, 2013, doi: 10.1007/s11633-013-0740-y.

[78] H. Tsutsui, J. Miura, and Y. Shirai, "Optical flow-based person tracking by multiple cameras," Multisens. Fusion Integr. Intell. Syst. 2001. MFI 2001. Int. Conf., no. May 2014, pp. 91-96, 2001, doi: 10.1109/MFI.2001.1013514.

[79] N. Dalal, B. Triggs, N. Dalal, B. Triggs, O. Gradients, and D. Cordelia, "Histograms of Oriented Gradients for Human Detection To cite this version: Histograms of Oriented Gradients for Human Detection," 2010.

[80] Q. Zhu, L. Chen, Q. Li, M. Li, A. Nüchter, and J. Wang, "3D LIDAR point cloud based intersection recognition for autonomous driving," IEEE Intell. Veh. Symp. Proc., pp. 456461, 2012, doi: 10.1109/IVS.2012.6232219.

[81] M. Arastounia, "Automated as-built model generation of subway tunnels from mobile Lidar data," Sensors (Switzerland), vol. 16, no. 9, 2016, doi: 10.3390/s16091486.

[82] M. Hämmerle, B. Höfle, and B. Höfle, "Effects of reduced terrestrial LiDAR point density on high-resolution grain crop surface models in precision agriculture," Sensors (Switzerland), vol. 14, no. 12, pp. 24212-24230, 2014, doi: 10.3390/s141224212.

[83] L. Yan, H. Liu, J. Tan, Z. Li, H. Xie, and C. Chen, "Scan line based road marking extraction from mobile LiDAR point clouds," Sensors (Switzerland), vol. 16, no. 6, pp. 1-21, 2016, doi: 10.3390/s16060903.

[84] M. Toews and T. Arbel, "Entropy-of-likelihood feature selection for image correspondence," Proc. Ninth IEEE Int. Conf. Comput. Vis., no. Iccv, pp. 1041-1047 vol.2, 2003, doi: 10.1109/ICCV.2003.1238464.

[85] C. H. Chan, "Fabric defect detection by Fourier analysis," IEEE Trans. Ind. Appl., vol. 36, no. 5, pp. 1267-1276, 2000, doi: 10.1109/28.871274.

[86] L. C. L. Chen, G. L. G. Lu, and D. Z. D. Zhang, "Effects of different Gabor filters parameters on image retrieval by texture," 10th Int. Multimed. Model. Conf. 2004. Proceedings., pp. 0-5, 2004, doi: 10.1109/MULMM.2004.1264996.

[87] C. Fernández-Isla, P. J. Navarro, and P. M. Alcover, "Automated visual inspection of ship hull surfaces using the wavelet transform," Math. Probl. ..., vol. 2013, p. 12, 2013, doi: $10.1155 / 2013 / 101837$. 
[88] Y. Q. Chen, M. S. Nixon, and D. W. Thomas, "Statistical geometrical features for texture classification," Pattern Recognit., vol. 28, no. 4, pp. 537-552, 1995, doi: 10.1016/00313203(94)00116-4.

[89] R. M. Haralick and K. Shanmugam, "04309314," 1973, doi: 10.1109/TSMC.1973.4309314.

[90] N. Aggarwal and R. K. Agrawal, "First and Second Order Statistics Features for Classification of Magnetic Resonance Brain Images," J. Signal Inf. Process., vol. 03, no. 02, pp. 146-153, 2012, doi: 10.4236/jsip.2012.32019.

[91] M.-K. Hu, "Visual pattern recognition by moment invariants," Inf. Theory, IEEE Trans., vol. 8, pp. 179-187, 1962, doi: 10.1109/TIT.1962.1057692.

[92] C. Robert, "Machine Learning, a Probabilistic Perspective," Chance, vol. 27, no. 2, pp. 6263, 2014, doi: 10.1080/09332480.2014.914768.

[93] www.allitebooks.com. .

[94] B. Lu, Q. Li, and W. Y. Liu, "Dynamic structure of the Sarcin/Ricin domain in rat 28S ribosomal RNA investigated by hybridization with oligodeoxynucleotide," Biol. Chem., vol. 378, no. 7, pp. 697-699, 1997, doi: 10.1515/bchm.1997.378.7.697.

[95] D. J. Hand, "Measuring classifier performance: A coherent alternative to the area under the ROC curve," Mach. Learn., vol. 77, no. 1, pp. 103-123, 2009, doi: 10.1007/s10994-009-51195 .

[96] G. D. Hager, G. Grunwald, and K. Toyama, "Intelligent Robots and Systems," Intell. Robot. Syst., vol. 1, pp. 415-430, 2007, doi: 10.1016/B978-044482250-5/50028-1.

[97] R. Fox, "No $\{\{$ Title $\}\}, "$ vol. 5, no. 1976, pp. 265-288, 2010.

[98] "Navarro-Serment, L.E.; Mertz, C.; Hebert, M. Pedestrian Detection and Tracking Using Three-dimensional LADAR Data. Int. J. Robot. Res. 2010 , 29, 1516-1528,” p. 2010, 2010.

[99] D. Corporation, "Pedestrian Detection and Tracking using in-vehicle Lidar," no. Iv, pp. 734-739, 2011, doi: 10.1109/IVS.2011.5940555.

[100] K. TAKAGI, S. ANDO, and M. HASHIMOTO, "Pedestrian Recognition Using On-vehicle LIDAR," Proceedings. JSAE Annu. Congr., no. Iv, pp. 9-12, 2006. 


\section{APENDICES}

\section{I.I CARACTERISTICAS RENAULT TWIZY}
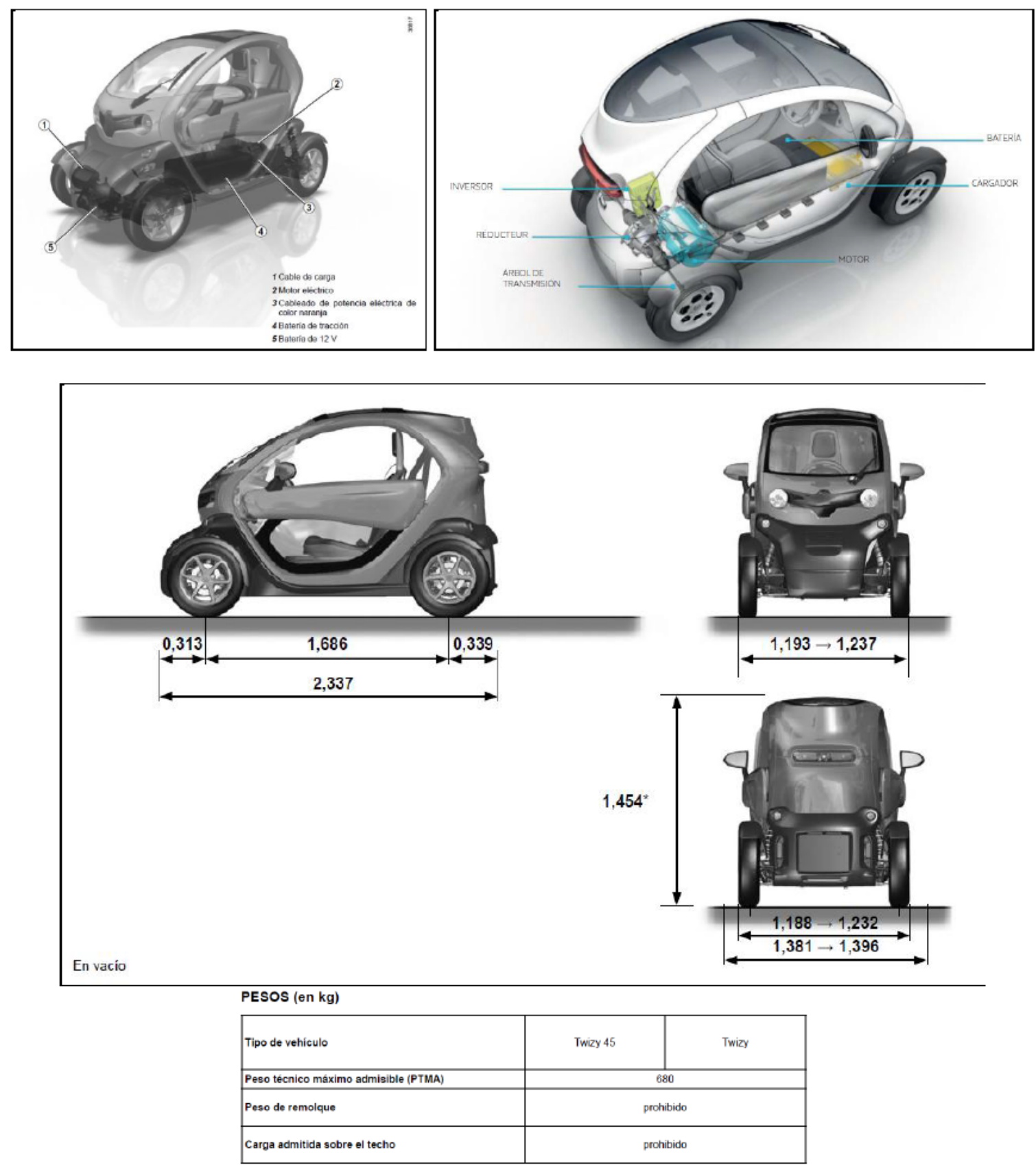


\section{I.II ESQUEMA UNIFILAR RENAULT TWIZY}

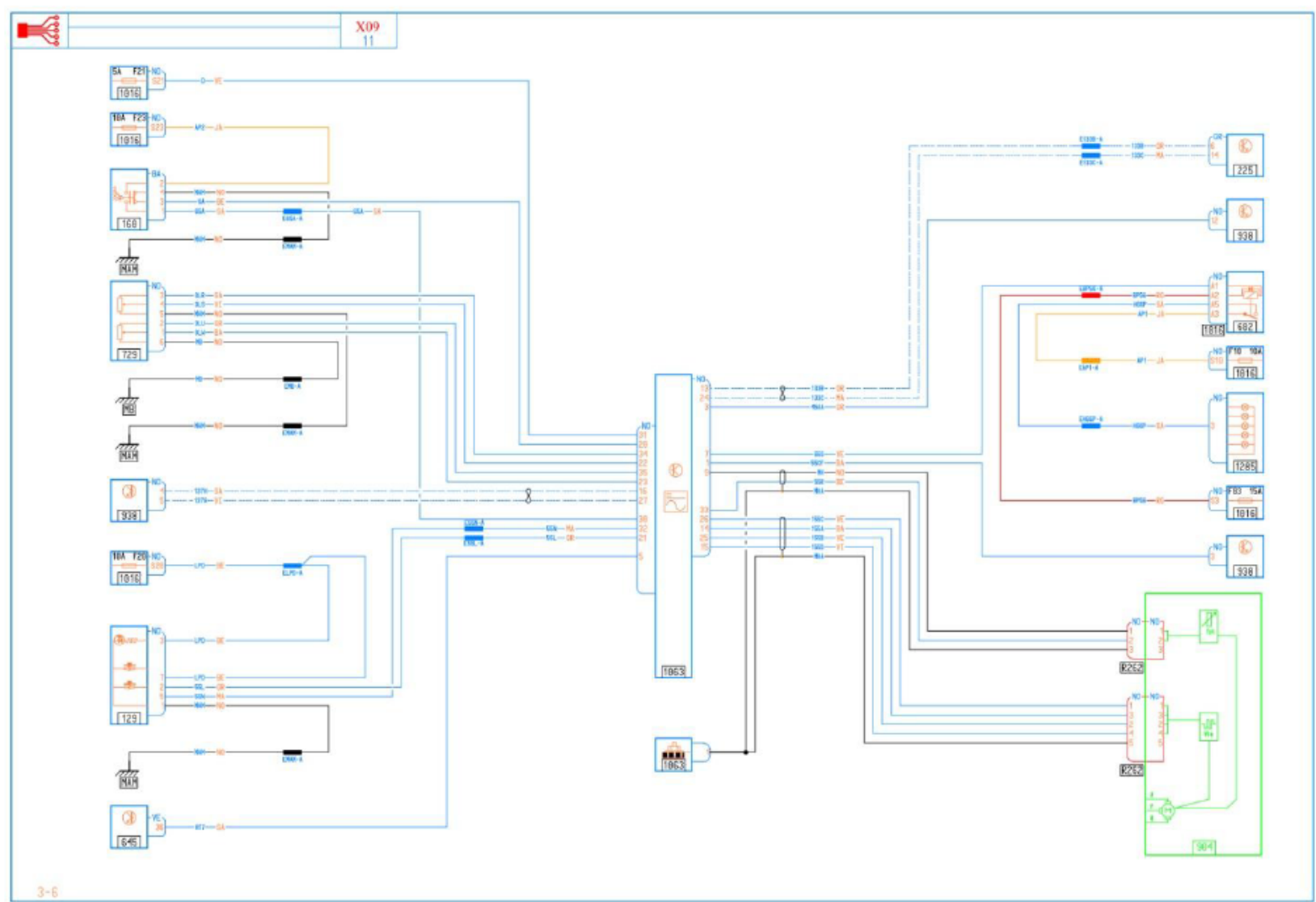




\section{I.III HOJAS DE CARACTERISTICAS DEL HARDWARE INSTALADO}

M 1:2.5
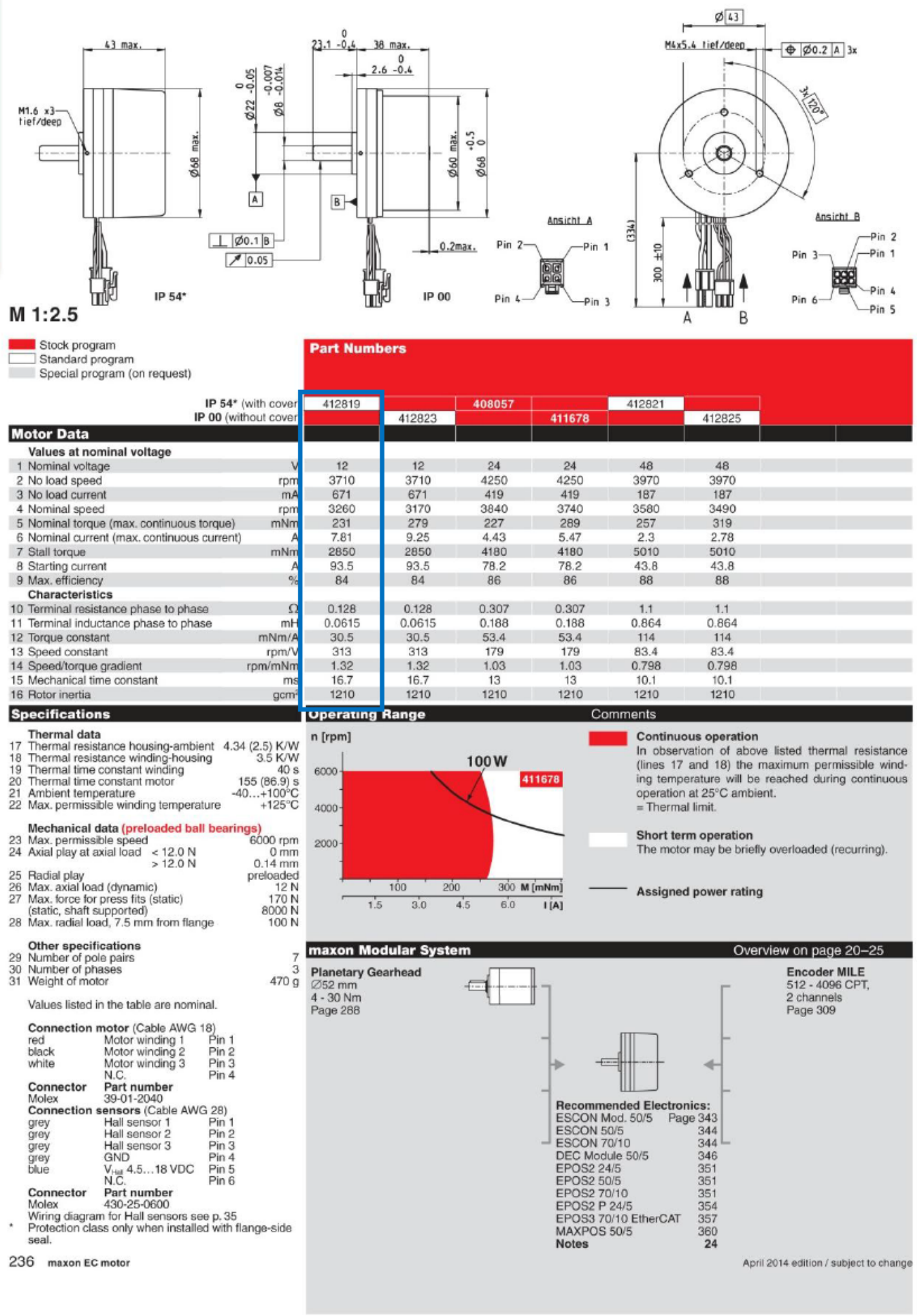
I.III.II REDUCTOR

Planetary Gearhead GP 52 C $\varnothing 52$ mm, 4-30 Nm

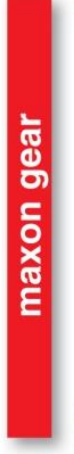

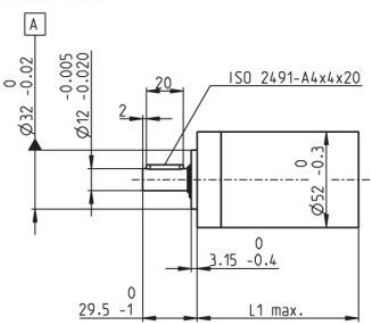

Stock program

Standard program

Special program (on request)

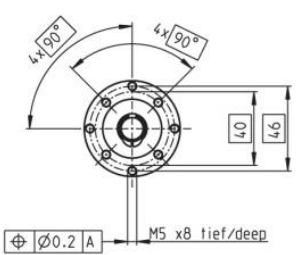

M 1:4

\section{Technical Data}

Planetary Gearhead

Output shaft

stainless stee

Radial play, $12 \mathrm{~mm}$ from flange preloaded ball bearings xial play at axial load $<5 \mathrm{~N} \quad \max .0 .06 \mathrm{~mm}$ Max. permissible axialload $>5 \mathrm{~N} \quad \max .0 .3 \mathrm{~mm}$ Max. permissible force for press fits Sense of rotation, drive Recommended input speed

$<6000 \mathrm{rpm}$ $-15 \ldots+80^{\circ} \mathrm{C}$
-30 Extended range as option $\quad-40 \ldots+100^{\circ} \mathrm{C}$ Max. radial load, $12 \mathrm{~mm}$ from flange

\begin{tabular}{|c|c|c|c|c|c|c|c|c|c|}
\hline \multicolumn{3}{|c|}{ Special program (on request) } & \multirow{2}{*}{223080} & \multirow{2}{*}{223083} & \multirow{2}{*}{223089} & \multirow{2}{*}{223094} & \multirow{2}{*}{223097} & \multirow{2}{*}{223104} & \multirow{2}{*}{223109} \\
\hline & arhead Data & & & & & & & & \\
\hline 1 & Reduction & & $3.5: 1$ & $12: 1$ & $43: 1$ & $91: 1$ & $150: 1$ & $319: 1$ & $546: 1$ \\
\hline 2 & Reduction absolute & & $7 / 2$ & $49 / 4$ & $343 / 8$ & 91 & $2401 / 16$ & $637 / 2$ & 546 \\
\hline 10 & Mass inertia & $\mathrm{gcm}^{2}$ & 20.7 & 17.6 & 17.3 & 16.7 & 17.3 & 16.8 & 16.4 \\
\hline & Max. motor shaft diameter & $\mathrm{mm}$ & 10 & 10 & 10 & 10 & 10 & 10 & 10 \\
\hline & Part Numbers & & 223081 & 223084 & 223090 & 223095 & 223099 & 223105 & 223110 \\
\hline 1 & Reduction & & $4.3: 1$ & $15: 1$ & $53: 1$ & $113: 1$ & $186: 1$ & $353: 1$ & $676: 1$ \\
\hline 2 & Reduction absolute & & $13 / 3$ & $91 / 6$ & $637 / 12$ & $338 / 3$ & $445 \% / 24$ & $28561 / 81$ & 676 \\
\hline 10 & Mass inertia & $\mathrm{gcm}^{2}$ & 12 & 16.8 & 17.2 & 9.3 & 17.3 & 9.4 & 9.1 \\
\hline & Max. motor shaft diameter & $\mathrm{mm}$ & 8 & 10 & 10 & 8 & 10 & 8 & 8 \\
\hline & Part Numbers & & & 223085 & ZZ3OYI & 223096 & 223101 & 223106 & 223111 \\
\hline 1 & Reduction & & & $19: 1$ & $66: 1$ & $126: 1$ & $230: 1$ & $394: 1$ & $756: 1$ \\
\hline 2 & Reduction absolute & & & $16 \% / 9$ & $1183 / 18$ & 126 & $8281 / 36$ & $1183 / 3$ & 756 \\
\hline 10 & Mass inertia & $\mathrm{gcm}^{2}$ & & 9.5 & 16.7 & 16.4 & 16.8 & 16.7 & 16.4 \\
\hline & Max. motor shaft diameter & $\mathrm{mm}$ & & 8 & 10 & 10 & 10 & 10 & 10 \\
\hline & Part Numbers & & & 223086 & 223092 & 223098 & 223102 & 223107 & 223112 \\
\hline & Reduction & & & $21: 1$ & $74: 1$ & $156: 1$ & $257: 1$ & $441: 1$ & $936: 1$ \\
\hline & Reduction absolute & & & 21 & $147 / 2$ & 156 & $102 \% / 4$ & 441 & 936 \\
\hline 10 & Mass inertia & $\mathrm{gcm}^{2}$ & & 16.5 & 17.2 & 9.1 & 17.3 & 16.5 & 9.1 \\
\hline & Max. motor shaft diameter & $\mathrm{mm}$ & & 10 & 10 & 8 & 10 & 10 & 8 \\
\hline & Part Numbers & & & 223087 & 223093 & & 223103 & 223108 & \\
\hline & Reduction & & & $26: 1$ & $81: 1$ & & $285: 1$ & $488: 1$ & \\
\hline & Reduction absolute & & & 26 & $2197 / 27$ & & $15379 / 54$ & $4394 / 9$ & \\
\hline 10 & Mass inertia & $\mathrm{gcm}^{2}$ & & 9.1 & 9.4 & & 16.7 & 9.4 & \\
\hline & Max. motor shaft diameter & $\mathrm{mm}$ & & 8 & 8 & & 10 & 8 & \\
\hline & Number of stages & & 1 & 2 & 3 & 3 & 4 & 4 & 4 \\
\hline & Max. continuous torque & $\mathrm{Nm}$ & 4 & 15 & 30 & 30 & 30 & 30 & 30 \\
\hline & Intermittently permissible torque at gear output & $\mathrm{Nm}$ & 6 & 22.5 & 45 & 45 & 45 & 45 & 45 \\
\hline & Max. efficiency & $\%$ & 91 & 83 & 75 & 75 & 68 & 68 & 68 \\
\hline & Weight & g & 460 & 620 & 770 & 770 & 920 & 920 & 920 \\
\hline & Average backlash no load & & 0.6 & 0.8 & 1.0 & 1.0 & 1.0 & 1.0 & 1.0 \\
\hline 11 & Gearhead length L1 & $\mathrm{mm}$ & 49.0 & 65.0 & 78.5 & 78.5 & 92.0 & 92.0 & 92.0 \\
\hline
\end{tabular}

11 Gearhead length L1

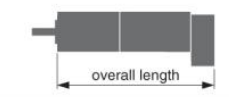

\section{maxon Modular System}

$\stackrel{\text { overall length }}{\longrightarrow}$ overall length

+ Page

EC 45, $150 \mathrm{~W} \quad 194$

E $45,150 \mathrm{~W}$

194

EC $45,250 \mathrm{~W} \quad 19$

EC 45, $250 \mathrm{~W}$

EC 45, 250 W 195

EC $45,250 \mathrm{~W} \quad 195$

EC-max 40, $120 \mathrm{~W} 207$

EC-max 40, 120 W 207

EC-max 40, 120 W 207

EC-max 40, $120 \mathrm{~W} 207$

EC 60 flat, IP 00 236

EC 60 flat, IP 54

EC 60 flat, IP 00

EC 60 flat, IP $54 \quad 236$

HEDL 9140

HEDL 9140

HEDL 9140

HEDL 9140

$\begin{array}{lll}\text { MR } & 320 & 137 . \\ \text { HEDL 5540 } & 331 & 1530\end{array}$

\begin{tabular}{l|l|l} 
& & \\
AB 28 & 371 & 160.5 \\
\hline & 371 & 171.5
\end{tabular}

$\begin{array}{lllll}\text { HEDL 5540 } & 331 & \text { AB 28 } & 371 & 189.8\end{array}$

\begin{tabular}{ll} 
MILE & 94.8 \\
\hline
\end{tabular}

MILE

-

\begin{tabular}{|c|c|c|c|c|c|c|}
\hline 160.3 & 176.3 & 189.8 & 189.8 & 203.3 & 203.3 & 203.3 \\
\hline 175.9 & 191.9 & 205.4 & 205.4 & 218.9 & 218.9 & 218.9 \\
\hline 160.3 & 176.3 & 189.8 & 189.8 & 203.3 & 203.3 & 203.3 \\
\hline 167.7 & 183.7 & 197.2 & 197.2 & 210.7 & 210.7 & 210.7 \\
\hline 184.7 & 200.7 & 214.2 & 214.2 & 227.7 & 227.7 & 227.7 \\
\hline 193.1 & 209.1 & 222.6 & 222.6 & 236.1 & 236.1 & 236.1 \\
\hline 208.7 & 224.7 & 238.2 & 238.2 & 251.7 & 251.7 & 251.7 \\
\hline 193.1 & 209.1 & 222.6 & 222.6 & 236.1 & 236.1 & 236.1 \\
\hline 200.5 & 216.5 & 230.0 & 230.0 & 243.5 & 243.5 & 243.5 \\
\hline 217.5 & 233.5 & 247.0 & 247.0 & 260.5 & 260.5 & 260.5 \\
\hline 137.1 & 153.1 & 166.6 & 166.6 & 180.1 & 180.1 & 180.1 \\
\hline 153.0 & 169.0 & 182.5 & 182.5 & 196.0 & 196.0 & 196.0 \\
\hline 160.5 & 176.5 & 190.0 & 190.0 & 203.5 & 203.5 & 203.5 \\
\hline 171.5 & 187.5 & 201.0 & 201.0 & 214.5 & 214.5 & 214.5 \\
\hline 189.8 & 205.8 & 219.3 & 219.3 & 232.8 & 232.8 & 232.8 \\
\hline 89.8 & 105.8 & 119.3 & 119.3 & 132.8 & 132.8 & 132.8 \\
\hline 94.8 & 110.8 & 124.3 & 124.3 & 137.8 & 137.8 & 137.8 \\
\hline 90.8 & 106.8 & 120.3 & 120.3 & 133.8 & 133.8 & 133.8 \\
\hline 94.8 & 110.8 & 124.3 & 124.3 & 137.8 & 137.8 & 137.8 \\
\hline 81.0 & 97.0 & 110.5 & 110.5 & 124.0 & 124.0 & 124.0 \\
\hline
\end{tabular}


I.III.III ENCODER

Encoder MILE 512-4096 CPT, 2 Channels, with Line Driver Integrated into motor
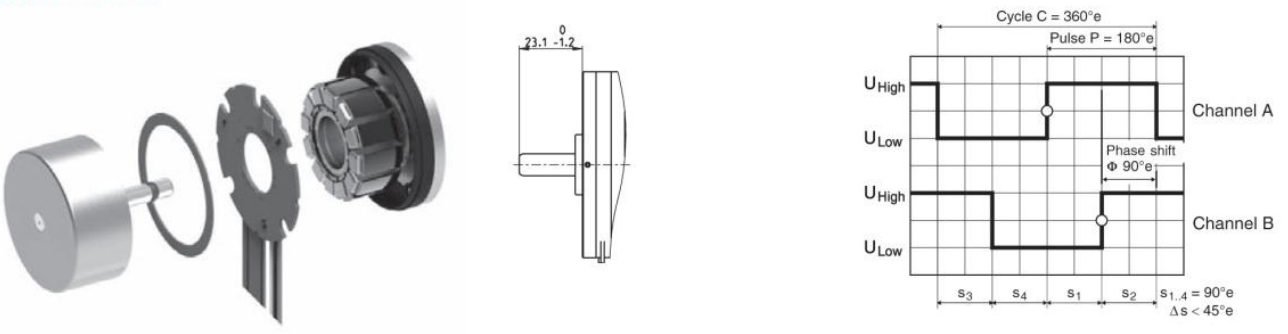

Direction of rotation $\mathrm{cw}$ (definition $\mathrm{cw} \mathrm{p} .78$ )
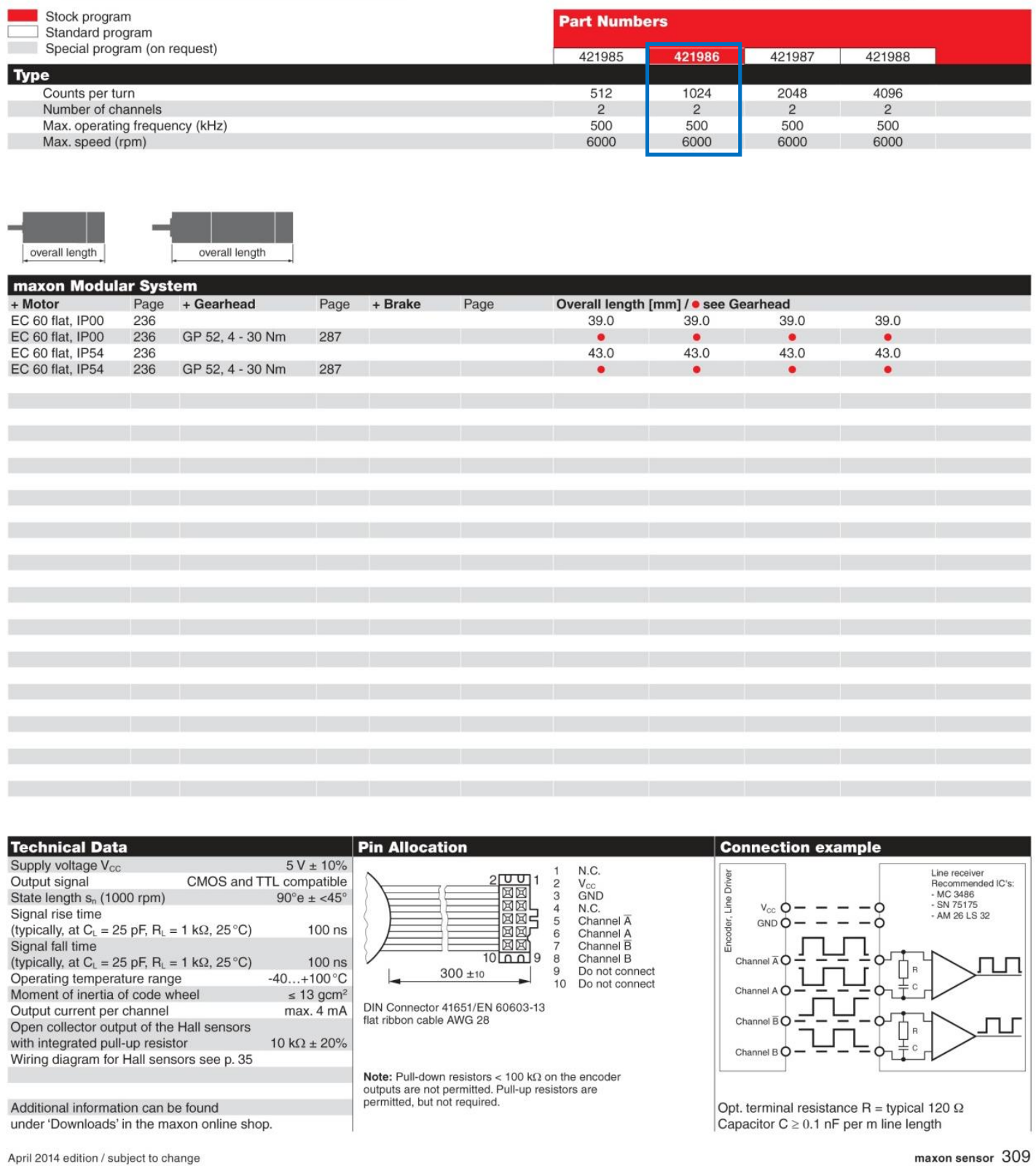

N

\}

级

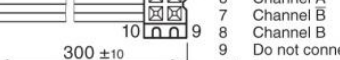

9 Do not connect
10 Do not connect

DiN Connector 41651/EN 60603-13

Note: Pull-down resistors $<100 \mathrm{k} \Omega$ on the encoder outputs are not permitted. Pull-up resistors are permitted, bot not required.

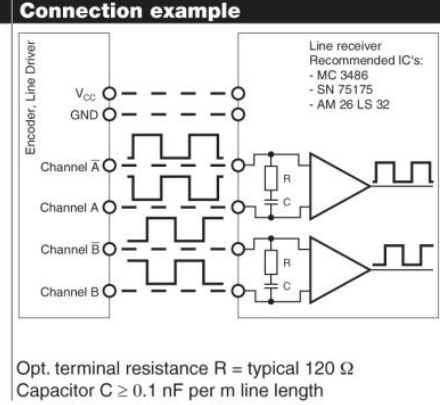

maxon sensor 309 


\section{I.III.IV DRIVER}

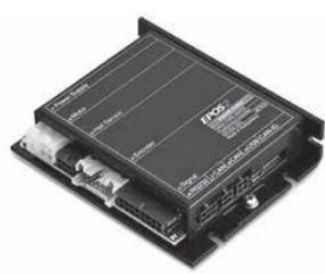

EPOS2 24/5

Matched with DC brush motors with encoder or brushless EC motors with Hall sensors and encoder to $120 / 240$ watts.

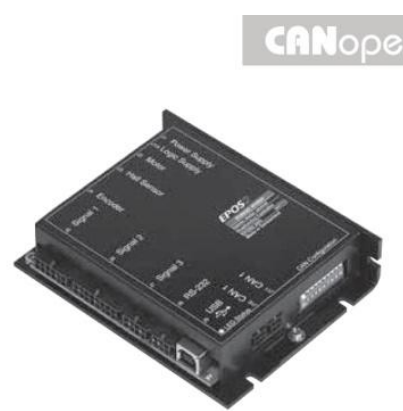

EPOS2 50/5

Matched with DC brush motors with encoder or brushless EC motors with Hall sensors and encoder to $250 / 500$ watts.
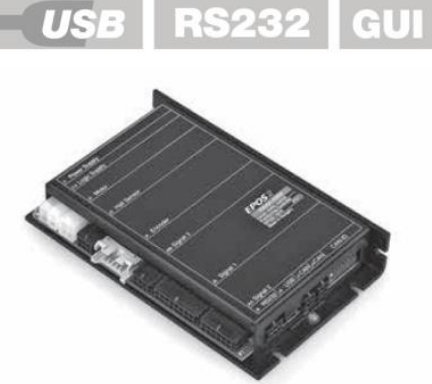

EPOS2 70/10

Matched with DC brush motors with encoder or brushless EC motors with Hall sensors or encoder to $700 / 1750$ watts.

\begin{tabular}{|c|c|c|}
\hline CANopen Slave & CANopen Slave & CANopen Slave \\
\hline \multicolumn{3}{|l|}{ Electrical data } \\
\hline $11-24$ VDC & $11-50 \mathrm{VDC}$ & $11-70 \mathrm{VDC}$ \\
\hline $11-24$ VDC & $11-50$ VDC & $11-70 \mathrm{VDC}$ \\
\hline $0.9 \times V_{C C}$ & $0.9 \times V_{C C}$ & $0.9 \times V_{C C}$ \\
\hline $10 \mathrm{~A}$ & $10 \mathrm{~A}$ & $25 \mathrm{~A}$ \\
\hline $5 \mathrm{~A}$ & $5 \mathrm{~A}$ & $10 \mathrm{~A}$ \\
\hline $50 \mathrm{kHz}$ & $50 \mathrm{kHz}$ & $50 \mathrm{kHz}$ \\
\hline $10 \mathrm{kHz}$ & $10 \mathrm{kHz}$ & $10 \mathrm{kHz}$ \\
\hline $1 \mathrm{kHz}$ & $1 \mathrm{kHz}$ & $1 \mathrm{kHz}$ \\
\hline $1 \mathrm{kHz}$ & $1 \mathrm{kHz}$ & $1 \mathrm{kHz}$ \\
\hline 25000 rpm (sinusoidal); 100000 rpm (block) & 25000 rpm (sinusoidal); 100000 rpm (block) & 25000 rpm (sinusoidal); 100000 rpm (block) \\
\hline $15 \mu \mathrm{H} / 5 \mathrm{~A}$ & $22 \mu \mathrm{H} / 5 \mathrm{~A}$ & $25 \mu \mathrm{H} / 10 \mathrm{~A}$ \\
\hline \multicolumn{3}{|l|}{ Input } \\
\hline $\mathrm{H} 1, \mathrm{H} 2, \mathrm{H} 3$ & $\mathrm{H} 1, \mathrm{H} 2, \mathrm{H} 3$ & $\mathrm{H} 1, \mathrm{H} 2, \mathrm{H} 3$ \\
\hline $\mathrm{A}, \mathrm{A} \backslash, \mathrm{B}, \mathrm{B} \backslash, \mathrm{I}, \mathrm{I}(\max .5 \mathrm{MHz})$ & $\mathrm{A}, \mathrm{Al}, \mathrm{B}, \mathrm{B} \backslash, \mathrm{I}, \mathrm{\perp}(\max .5 \mathrm{MHz})$ & $\mathrm{A}, \mathrm{Al}, \mathrm{B}, \mathrm{B} \backslash, \mathrm{I}, \mathrm{I}(\max .5 \mathrm{MHz})$ \\
\hline 6 (TTL and PLC level) & 11 (7 optically isolated, 4 differential) & 10 (7 optically isolated, 3 differential) \\
\hline 2 (12-bit resolution, $0 \ldots+5 \mathrm{~V}$ ) & 2 (differential, 12-bit resolution, $\pm 10 \mathrm{~V}$ ) & 2 (differential, 12 -bit resolution, $0 \ldots+5 \mathrm{~V}$ ) \\
\hline configurable with DIP switch $1 \ldots 7$ & configurable with DIP switch $1 \ldots 7$ & configurable with DIP switch $1 \ldots 7$ \\
\hline \multicolumn{3}{|l|}{ Output } \\
\hline \multirow[t]{2}{*}{4} & 5 (4 optically isolated, 1 differential) & 5 (4 optically isolated, 1 differential) \\
\hline & 1 (12-bit, $0 \ldots 10 \mathrm{~V})$ & \\
\hline$+5 \mathrm{VDC}, \max 100 \mathrm{~mA}$ & $+5 \mathrm{VDC}$, max. $100 \mathrm{~mA}$ & $+5 \mathrm{VDC}, \max .100 \mathrm{~mA}$ \\
\hline$+5 \mathrm{VDC}$, max. $30 \mathrm{~mA}$ & $+5 \mathrm{VDC}, \max .30 \mathrm{~mA}$ & $+5 \mathrm{VDC}, \max .30 \mathrm{~mA}$ \\
\hline $\mathrm{V}_{\mathrm{cc}}, \max .1300 \mathrm{~mA}$ & $+5 \mathrm{VDC}, \max .150 \mathrm{~mA}$ & $+5 \mathrm{VDC}$, max. $150 \mathrm{~mA} ;+5 \mathrm{VDC}\left(\mathrm{R}_{\mathrm{i}}=1 \mathrm{k} \Omega\right)$ \\
\hline \multicolumn{3}{|l|}{ Interface } \\
\hline RxD; TxD (max. 115200 bit/s) & RxD; TxD (max. 115200 bit/s) & RxD; TxD (max. 115200 bit/s) \\
\hline high; low (max. $1 \mathrm{Mbit} / \mathrm{s})$ & high; low (max. $1 \mathrm{Mbit} / \mathrm{s})$ & high; low (max. $1 \mathrm{Mbit} / \mathrm{s})$ \\
\hline Data+; Data- (max. $12 \mathrm{Mbit} / \mathrm{s})$ & Data+; Data- (max. $12 \mathrm{Mbit} / \mathrm{s})$ & Data+; Data- (max.12 Mbit/s) \\
\hline \multicolumn{3}{|l|}{ Indicator } \\
\hline green LED, red LED & green LED, red LED & green LED, red LED \\
\hline \multicolumn{3}{|l|}{ Environmental conditions } \\
\hline$-10 \ldots+55^{\circ} \mathrm{C}$ & $-10 \ldots+45^{\circ} \mathrm{C}$ & $-10 \ldots+45^{\circ} \mathrm{C}$ \\
\hline$+55 \ldots+83^{\circ} \mathrm{C}$; Derating: $-0.179 \mathrm{~A}^{\circ} \mathrm{C}$ & $+45 \ldots+80^{\circ} \mathrm{C}$; Derating: $-0.143 \mathrm{~A}^{\circ} \mathrm{C}$ & $+45 \ldots+85^{\circ} \mathrm{C}$; Derating: $-0.250 \mathrm{~A} /{ }^{\circ} \mathrm{C}$ \\
\hline$-40 \ldots+85^{\circ} \mathrm{C}$ & $-40 \ldots+85^{\circ} \mathrm{C}$ & $-40 \ldots+85^{\circ} \mathrm{C}$ \\
\hline $5 \ldots 90 \%$ & $5 \ldots 90 \%$ & $5 \ldots 90 \%$ \\
\hline \multicolumn{3}{|l|}{ Mechanical data } \\
\hline Approx. $170 \mathrm{~g}$ & Approx. $240 \mathrm{~g}$ & Approx. $330 \mathrm{~g}$ \\
\hline $105 \times 83 \times 24 \mathrm{~mm}$ & $120 \times 93.5 \times 27 \mathrm{~mm}$ & $150 \times 93 \times 27 \mathrm{~mm}$ \\
\hline Flange for M3-screws & Flange for M3-screws & Flange for M3-screws \\
\hline \multicolumn{3}{|l|}{ Part numbers } \\
\hline 367676 EPOS2 24/5 & 347717 EPOS2 50/5 & 375711 EPOS2 70/10 \\
\hline \multicolumn{3}{|l|}{ Accessories } \\
\hline 309687 DSR 50/5 Shunt regulator & 309687 DSR 50/5 Shunt regulator & 235811 DSR 70/30 Shunt regulator \\
\hline Order accessories separately, see page 449 & Order accessories separately, see page 449 & Order accessories separately, see page 449 \\
\hline
\end{tabular}


I.III.V LASER 3D VELODYINE LIDAR

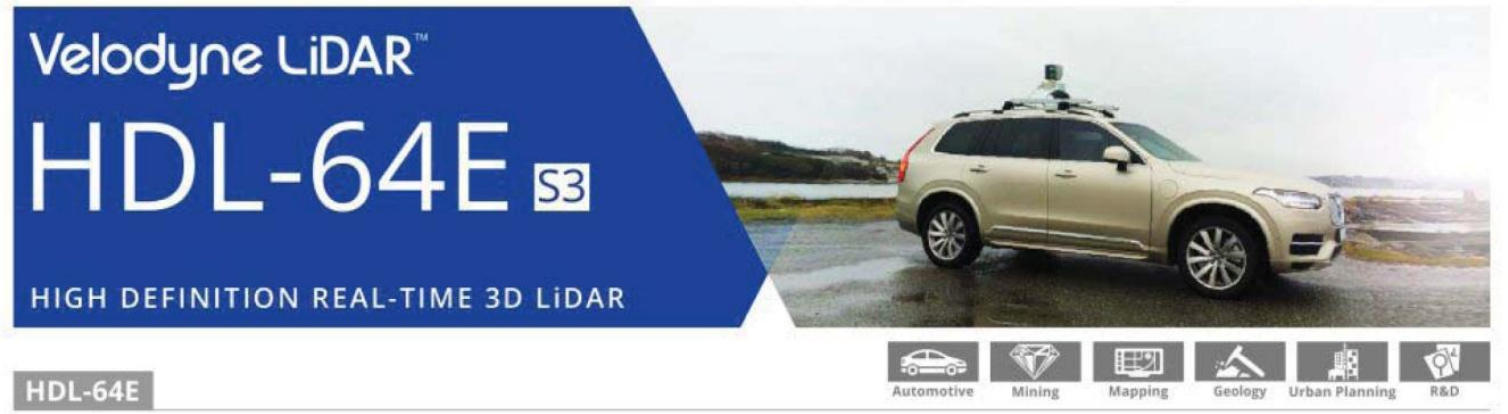

\section{Real-Time 3D LiDAR}

The HDL-64E S3 is Velodyne's high resolution and performance LiDAR sensor product that supports single or dual returns. Similar to its predecessor, the S2, it captures high definition, real-time 3D information about the surrounding environment. It is ideal for applications such as autonomous vehicle navigation, 3D mapping and surveying plus industrial automation.

\section{Wide Field of View and High Frame Rate}

The HDL-64E S3 provides both unprecedented and unmatched resolution and field of views to generate a wealth of data about the surrounding environment. It utilizes 64 LiDAR channels aligned from $+2.0^{\circ}$ to $-24.9^{\circ}$ for a vertical field of view of $26.9^{\circ}$ and similar to the S2 model it delivers a real-time $360^{\circ}$ horizontal field of view with its patented rotating head design. The rotation rate is user-selectable from $5 \mathrm{~Hz}$ to $20 \mathrm{~Hz}$ to enable the user to determine the density of data points generated by the LiDAR sensor. The HDL-64E S3 generates a point cloud of up to 2,200,000 points per second with a range of up to $120 \mathrm{~m}$ and a typical accuracy of $\pm 2 \mathrm{~cm}$. The HDL-64E S3 is designed to operate over a wide temperature range $\left(-10^{\circ} \mathrm{C}\right.$ to $\left.+60^{\circ} \mathrm{C}\right)$ and challenging environments to support diverse operating conditions and applications.

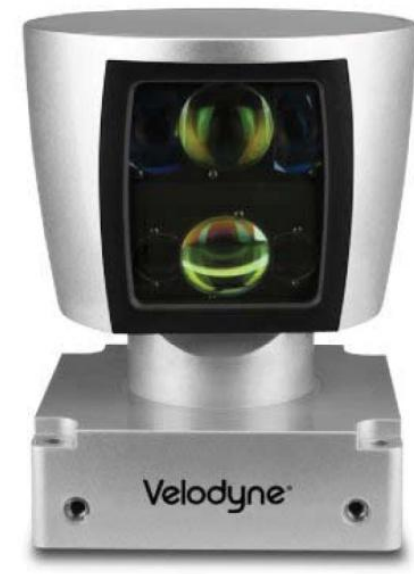

\section{DIMENSIONS}

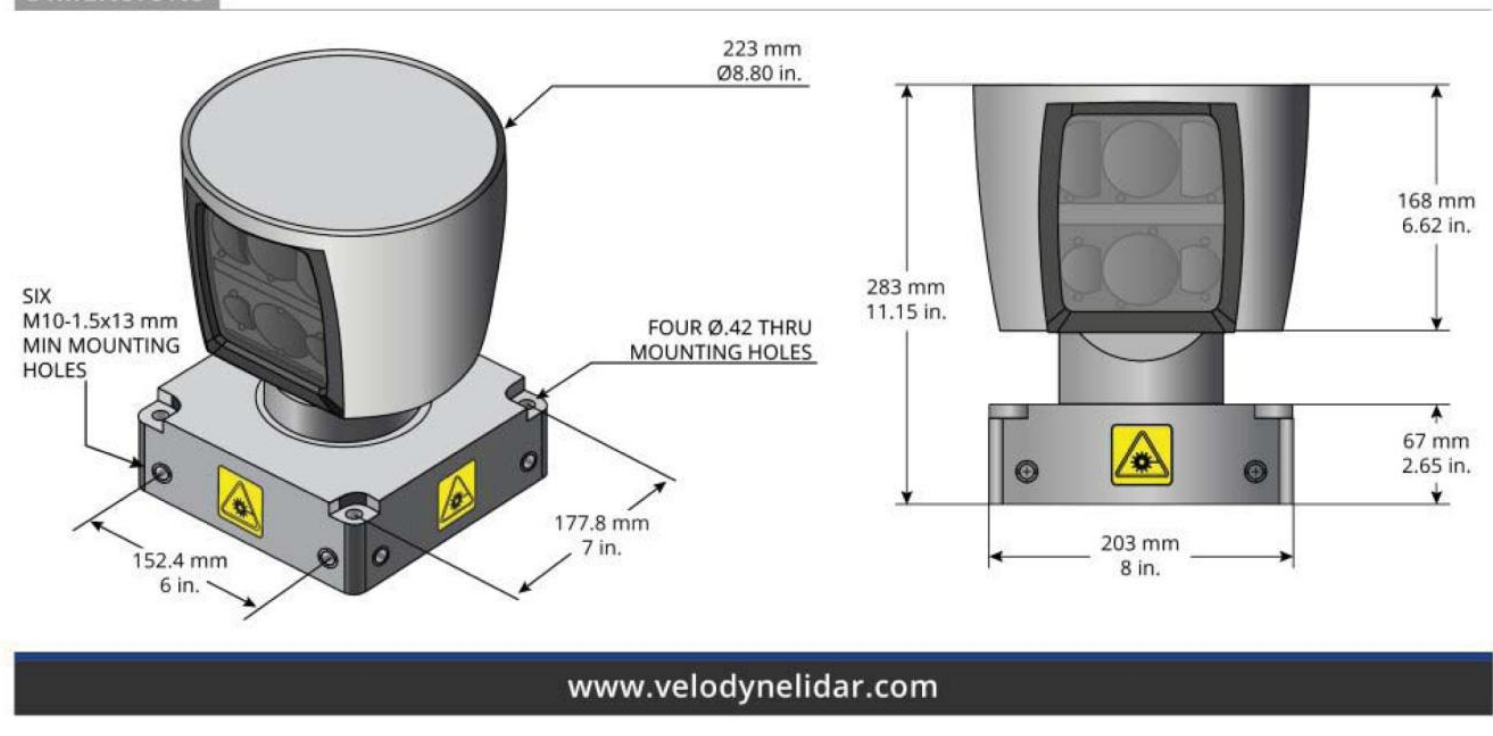


High Definition LiDAR Sensor

The HDL-64E S3 provides high definition 3 dimensional information about the surrounding environment.

\section{Specifications:}

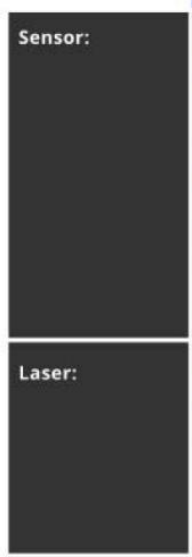

- Time of Flight Distance Measurement with Intensity

. 64 channels

- Measurement Range: Up to $120 \mathrm{~m}$

- Accuracy: $\pm 2.0 \mathrm{~cm}$ (Typical)

- Single or Dual Returns

- Field of View (Vertical): $+2.0^{\circ}$ to $-24.9^{\circ}\left(26.9^{\circ}\right)$

- Angular Resolution (Vertical): $0.4^{\circ}$

- Field of View (Horizontal): $360^{\circ}$

- Angular Resolution (Horizontal/Azimuth): $0.08^{\circ}-0.35^{\circ}$

- Rotation Rate: $5 \mathrm{~Hz}-20 \mathrm{~Hz}$

- Laser Product Classification: Class 1 Eye-safe

- Wavelength: $903 \mathrm{~nm}$

- Beam Size:

Upper Block: $20.0 \mathrm{~mm} \times 10.0 \mathrm{~mm}$

Lower Block: $15.0 \mathrm{~mm} \times 10.0 \mathrm{~mm}$

- Dynamic Laser Power Selection for Larger Dynamic Range

Mechanical/

Electrical/

- Power Consumption: $60 \mathrm{~W}$ (Typical)

Operational

- Operating Voltage: $12 \mathrm{~V}-32 \mathrm{~V}$

- Weight: $28 \mathrm{lbs}$. (12.7 Kg) (without cabling)

- Dimensions: $215 \mathrm{~mm}$ Diameter $\times 283 \mathrm{~mm}$ Height (Base: $203 \mathrm{~mm} \times 203 \mathrm{~mm}$ )

- Operating Temperature: $-10^{\circ} \mathrm{C}$ to $+60^{\circ} \mathrm{C}$

- Storage Temperature: $-40^{\circ} \mathrm{C}$ to $+85^{\circ} \mathrm{C}$

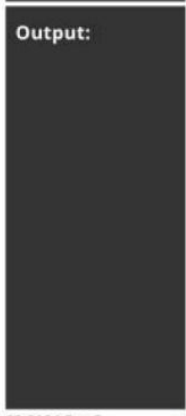

-3D LIDAR Data Points Generated:

Single Return Mode: $\sim 1,300,000$ points per second

Dual Return Mode: $\quad-2,200,000$ points per second

100 Mbps Ethernet Connection

- UDP Packets Contain:

Time of Flight Distance Measurement

- Intensity Measurement

- Rotation Angles

Synchronized Time Stamps (us resolution)

- GPS: \$GPRMC NMEA Sentence from GPS Receiver (GPS not included)

63.9194 Rev-F Product Ordering Information:

Product Order

Product Name

SKU Ordering Number

Connector Type

HDL-64E S3 Dual Return Mode

80-HDL64E S3D

RJ45 + RS232

HDL-64E S3 Single Return Mode

80-HDL64E S3S 
I.III.Vı UNIDADES DE MEDIDAS INERCIALES IMU

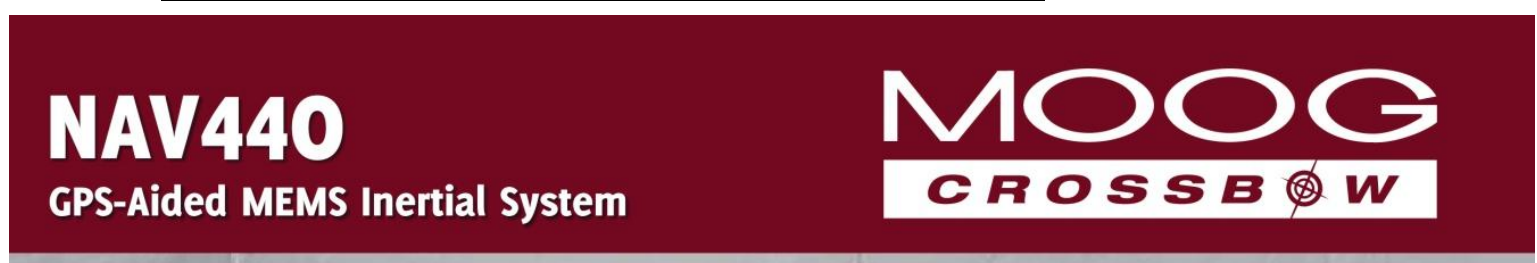

\section{DESCRIPTION}

The Moog Crossbow NAV440 is a fully-integrated combined GPS navigation and GPS-aided Attitude \& Heading Reference System (AHRS) solution. The NAV440 provides full inertial data (angles, rates, accels) and GPS position, along with inertially computed velocity that provides significant improvement in stability and higher data rates compared with stand-

alone GPS velocity measurements.

The NAV440 integrates highly reliable MEMS sensors (gyros and accelerometers), 3-axis magnetometer, and a WAAS/EGNOS-enabled GPS receiver all in a compact and rugged environmentally sealed enclosure. The NAV440 provides consistent performance in challenging operating environments, and is user-configurable for a wide variety of applications such as unmanned vehicle control, land vehicle guidance, avionics systems, and platform stabilization.

\section{KEY FEATURES}

- Pitch and roll accuracy of $<0.4^{\circ}$

- Output data rate up to $100 \mathrm{~Hz}$

- WAAS and EGNOS enable GPS

- Low power $<4 \mathrm{~W}$ at $28 \mathrm{VDC}$

- High reliability, MTBF >25,000 hours

- Rugged sealed enclosure

- Certified for DO-160D environments

\section{SPECIFICATIONS}

\section{Environment}

Operating Temperature

Enclosure

$-40^{\circ}$ to $+71^{\circ} \mathrm{C}$

\section{Electrical}

Input Voltage

Power Consumption

Digital Interface

Physical

Size

Weight

Interface Connector

GPS Antenna Connector

9 to 42 VDC

IP66 compliant

$<4 \mathrm{~W}$

RS-232

$3.0^{\prime \prime} \mathrm{w} \times 3.75^{\prime \prime} \mathrm{I} \times 3.0^{\prime \prime} \mathrm{h}$

$1.3 \mathrm{lbs}(0.58 \mathrm{~kg})$

DB15, D-sub 15 pin Male

SMA Male

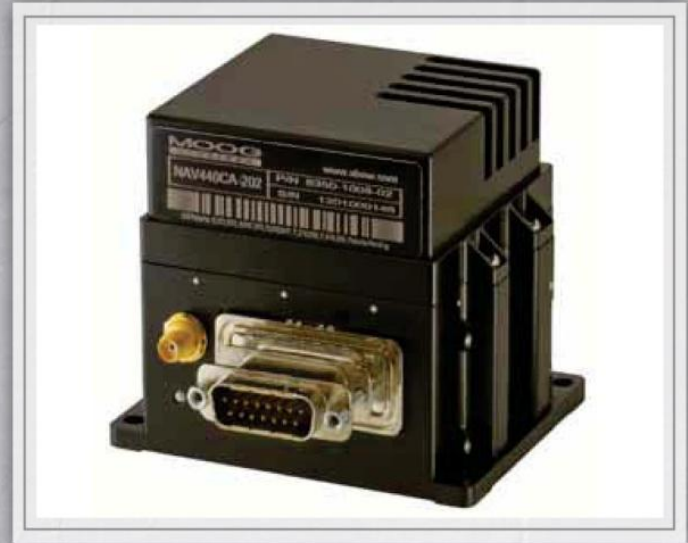

\section{PERFORMANCE}

Position/Velocity

Position Accuracy

1PPS Accuracy

Heading

Accuracy

\section{Attitude}

Range: Roll, Pitch

Accuracy

Angular Rate

Range: Roll, Pitch, Yaw

Bias Stability in run

Bias Stability over temp

Acceleration

Input Range

Bias Stability in run

Bias Stability over temp

$<3.0 \mathrm{~m}$ CEP

$\pm 50 \mathrm{~ns}$

$<1.0^{\circ} \mathrm{rms}$ (magnetic)

$<0.75^{\circ} \mathrm{rms}$ (with GPS aiding)

$\pm 180^{\circ}, \pm 90^{\circ}$

$<0.4^{\circ}$

$\pm 200^{\circ}$

$<10 \%$ hr

$<0.02 \%$ sec

$\pm 4 \mathrm{~g}$ or $\pm 10 \mathrm{~g}$

$<1 \mathrm{mg}$

$<4 \mathrm{mg}$

\section{ORDERING INFORMATION}

\section{Model Description}

NAV440CA-202

GPS-Aided MEMS Inertial System

Includes NAV-VIEW software, User's manual,

Quick start guide, GPS Antenna, and cable.

\section{For more information}

Phone: 1-408-965-3300

Email: sales@moog-crossbow.com

www.moog-crossbow.com

Moog Crossbow

1421 McCarthy Blvd.

Milpitas, CA 95035 


\section{I.III.VII SENSORES DE PROFUNDIDAD TOF}

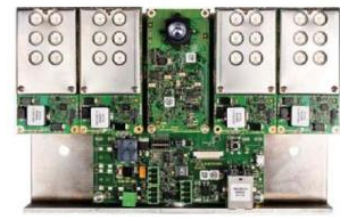

cope of supply bare sensor PON: 150-2224-1

Sentis ${ }^{30}-$ M420

Scope of supply package

PON: 150-2218-1

Sentis ${ }^{30}-$ M420

USB Cable

JTAG Adapter

Power Supply

Tripod

Documentation
The Sentis ${ }^{3 D}$ - M420 is a new depth sensor, operating on the Time-of-Flight (ToF) principle. The M420 is equipped with a PMD PhotonICs $®$ 19k-S3 Timeof-Flight 3D IC sensor.

The Depth Sensor Module with a powerful Illumination System achieves ranges up to $7 \mathrm{~m}$ with a $90^{\circ}$ Field-of-View. The Point Cloud streams the data over USB. The Sensor can be accessed through our Windows and Linux API.

Using active IR illumination, the sensor is able to capture 3D information. With a range of $7 \mathrm{~m}$ indoors, a field of view of $90^{\circ}$ and a size of only $205 \times 125 \times 85 \mathrm{~mm}$, this USB connected sensor can be used for next generation sensor systems in various application fields like robotics, automation and people counting

supply. $\begin{array}{ll}\text { Dimensions } & 205 \times 125 \times 85 \mathrm{~mm} \\ \text { Temperature } & -20 \text { to } 60{ }^{\circ} \mathrm{C}\end{array}$

Application Range up to $7 \mathrm{~m}$

Resolution $160 \times 120 \mathrm{px}$

\begin{tabular}{ll}
\hline FoV & $90^{\circ}$ \\
FPS & up to $160 \mathrm{fps}$
\end{tabular}

Illumination $850 \mathrm{~nm}$ LED

Operating Systems

Linux, ROS, Windows XP/7/8/10 32-64 bit

Frameworks

LabVIEW, MATLAB@, MetriCam, Halcon

Interfaces

$1 \times$ Trigger In,

$1 \times$ Trigger Out.

$1 \times$ GPIO,

$1 \times \mathrm{RS} 232$

$1 \times \mathrm{RS} 485$ 


\section{I.III.VIII ESCÁNER LÁSER 2D}

Detailed technical data

\section{Features}

\begin{tabular}{|c|c|c|c|c|}
\hline & & \\
\hline & TiM51x & TiM55x & TiM56x & TiM57x \\
\hline Light source & Infrared $(850 \mathrm{~nm})$ & & & \\
\hline Laser class & 1, eye-safe (IEC 6082 & $-1: 2014$ & & \\
\hline Aperture angle & $270^{\circ}$ & & & \\
\hline Scanning frequency & $15 \mathrm{~Hz}$ & & & \\
\hline Operating range & $0.05 \mathrm{~m} \ldots .4 \mathrm{~m}$ & $0.05 \mathrm{~m} \ldots 10 \mathrm{~m}$ & & $0.05 \mathrm{~m} \ldots 25 \mathrm{~m}$ \\
\hline Max. range with $10 \%$ reflectivity & $2 \mathrm{~m}$ & $8 \mathrm{~m}$ & & \\
\hline \multicolumn{5}{|l|}{ Performance } \\
\hline & TiM51x & TiM55x & TiM56x & TiM57x \\
\hline Response time & \multicolumn{4}{|l|}{ Typ. $67 \mathrm{~ms}$} \\
\hline Detectable object shape & \multicolumn{4}{|l|}{ Almost any } \\
\hline Systematic error ${ }^{1)}$ & $\pm 40 \mathrm{~mm}$ & $\pm 60 \mathrm{~mm}$ & & \\
\hline Statistical error ${ }^{1)}$ & $30 \mathrm{~mm}$ & $20 \mathrm{~mm}$ & & \\
\hline
\end{tabular}

1) Technical data such as measurement errors and sensing range can only be guaranteed up to a sensing range of $10 \mathrm{~m}$.

\section{Interfaces}

\begin{tabular}{|c|c|c|c|c|}
\hline & TiM51x & TiM55x & TiM56x & TiM57x \\
\hline Serial (RS-232) & $\checkmark$ & - & & \\
\hline Function & Host & - & & \\
\hline Data transmission rate & 115.2 Baud & - & & \\
\hline Ethernet & - & $\boldsymbol{v}$ & & \\
\hline USB & \multicolumn{4}{|l|}{$\boldsymbol{\nu}$, micro USB } \\
\hline Function & \multicolumn{4}{|l|}{ AUX, parameterization } \\
\hline Switching inputs & \multicolumn{4}{|l|}{0} \\
\hline Optical indicators & $\begin{array}{l}2 \text { LEDs (ON, switching } \\
\text { status) }\end{array}$ & \multicolumn{3}{|c|}{2 LEDs (ON, “device ready") } \\
\hline
\end{tabular}

\section{Mechanics/electronics}

\begin{tabular}{|c|c|c|c|c|}
\hline & TiM51x & TiM55x & TiM56x & TiM57x \\
\hline Operating voltage & \multicolumn{4}{|l|}{$9 \vee D C \ldots 28 \vee D C$} \\
\hline Power consumption & \multicolumn{4}{|l|}{ Typ. 4 W } \\
\hline Enclosure rating & $\begin{array}{l}\text { IP } 65 \\
\text { (IEC 60529:1989+A } \\
\text { MD1:1999+AMD2: } \\
\text { 2013) }\end{array}$ & \multicolumn{3}{|c|}{ IP 67 (IEC 60529:1989+AMD1:1999+AMD2:2013) } \\
\hline Protection class & \multicolumn{4}{|l|}{ III (IEC 61140:2016-1) } \\
\hline Weight & $\begin{array}{l}150 \mathrm{~g} \text {, without con- } \\
\text { necting cables }\end{array}$ & \multicolumn{3}{|c|}{$250 \mathrm{~g}$, without connecting cables } \\
\hline Dimensions $(\mathrm{L} \times \mathrm{W} \times \mathrm{H})$ & $\begin{array}{l}60 \mathrm{~mm} \times 60 \mathrm{~mm} \times \\
79 \mathrm{~mm}\end{array}$ & \multicolumn{3}{|c|}{$60 \mathrm{~mm} \times 60 \mathrm{~mm} \times 86 \mathrm{~mm}$} \\
\hline
\end{tabular}

Ambient data

\begin{tabular}{l} 
Electromagnetic compatibility (EMC) \\
\hline Vibration resistance
\end{tabular}

\section{TiM51x}

IEC 61000-6-3:2006

+AMD1:2010

IEC 61000-6-1:2016

IEC 60068-2-6:2007

07


